\title{
Soret and Diffusion Coefficients Measurement for Binary and Ternary Mixtures on Board the International Space Station Using SODI Apparatus
}

\author{
By \\ Amirhossein Ahadi \\ B.Sc. in Aerospace Engineering, KNTU, Tehran, Iran, 2010 \\ M.Sc. in Mechanical Engineering, Ryerson University, Toronto, ON, Canada 2011
}

A dissertation presented to Ryerson University

in partial fulfillment of the requirement for the degree of

DOCTOR OF PHILOSOPHY

In the Program of MECHANICAL AND INDUSTRIAL ENGINEERING

Toronto, Ontario, Canada, 2014

(C) (Amirhossein Ahadi) 2014 


\section{AUTHOR DECLARATION FOR ELECTRONIC SUBMISSION OF A DISSERTATION}

I hereby declare that I am the sole author of this thesis or dissertation.

I authorize Ryerson University to lend this thesis or dissertation to other institutions or individual for the purpose of scholarly research.

I further authorize Ryerson University to reproduce this thesis or dissertation by photocopying or by other means, in total or in part, at the request of other institutions or individuals for the purpose of scholarly research.

I understand that my dissertation may be made electronically available to the public. 


\title{
ABSTRACT
}

\section{Soret and Diffusion Coefficients Measurement for Binary and Ternary Mixtures on Board the International Space Station Using SODI Apparatus}

\author{
Doctor of Philosophy \\ Amirhossein Ahadi \\ Mechanical and Industrial Engineering, 2014 \\ Ryerson University, Toronto, ON, M5B 2K3, Canada
}

Thermodiffusion or Soret effect is a heat and mass transfer phenomenon in a non-isothermal liquid and gas mixtures. This phenomenon is more pronounced in oil fields, usually due to the porous environment. A precise and better understanding of the thermodiffusion phenomena in multi-component mixtures results in a more accurate modeling of oil reservoirs. Accordingly, the main objective of this study is to investigate the thermodiffusion phenomenon in the multicomponent mixtures. In order to achieve this objective, two series of thermodiffusion experiments conducted on board the International Space Station (ISS) using the SODI (Selectable Optical Diagnostics Instrument) facility were analyzed.

The first series of experiments aimed to study the effects of the forced vibration on the Soret phenomena. The experimental mixture was water and isopropanol with different compositions subjected to various temperature gradients normal to the vibrations. Results revealed maximum separation for the case with the minimum vibration and the lower temperature gradient; however, a linear relationship between Gershuni number and maximum separation was not found.

On the other hand, the second series of experiment was aimed to the measurement of the diffusion coefficients of selected ternary mixtures. Mixtures of tetrahydronaphthaleneisobutylbenzene-dodecane at five different compositions were hosted in the DSC (Diffusion and Soret Coefficient) cell array. Thus, the Soret diffusion coefficients and the molecular diffusion coefficients of the mentioned hydrocarbon mixture at five different compositions have been reported. 
To process the results of these experiments an advance image processing technique was developed and implemented in an application with GUI (Graphical User Interface) for the MachZehnder interferometer (MZI). Then, the application of the windowed Fourier transformation (WFT) to analyze the heat and mass transfer problem using the MZI setup is proposed. Results show that the WFT noticeably improves the measurement of concentration. This improvement is more evident for the ternary. It was shown that about $10 \%$ underestimation of the Soret coefficient would be resulted; if an accurate determination of the thermal time for the MZI is not used. The reliability and the repeatability of the MZI apparatus on board ISS to study thermodiffusion for binary and ternary mixtures were shown. 


\section{BORROWER}

Ryerson University requires the signatures of all persons using or photocopying this thesis.

Please sign below, and give address and date. 


\section{ACKNOWLEDGEMENTS}

I would like to thank my supervisor, Prof. Ziad Saghir, who has provided me full support during my MSc and $\mathrm{PhD}$. I am grateful to him for his valuable guide. Without his guidance and his support, it was impossible to complete this thesis.

The author also acknowledges the financial support by the Canadian Space Agency and Natural Sciences \& Engineering Research Council of Canada (NSERC) and also the useful suggestions of his colleagues in the Microgravity Research Lab at Ryerson University.

Through my parents' constant love, caring advice, and honesty, I could continue my studies, and do research. I would like to express my gratitude to my fiancé Sogol, and my sisters Faezeh and Faegheh. I thank them all for their love, patience, and support. I want to dedicate this thesis to all of them. 


\section{Table of Contents}

AUTHOR DECLARATION FOR ELECTRONIC SUBMISSION OF A DISSERTATION .....................ii

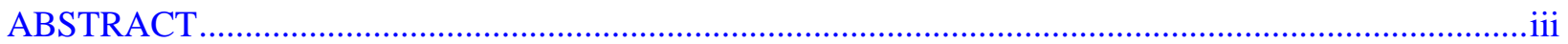

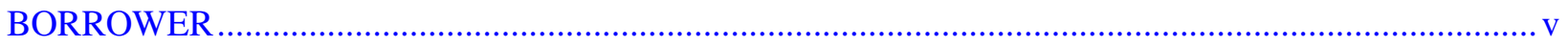

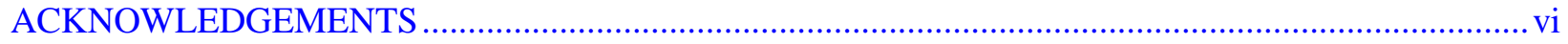

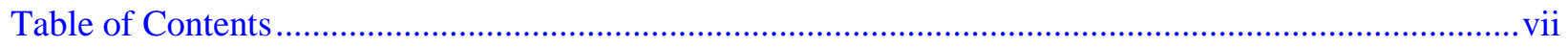

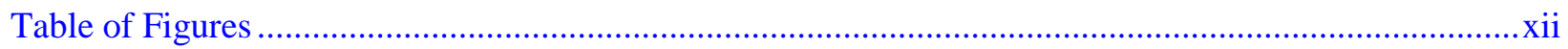

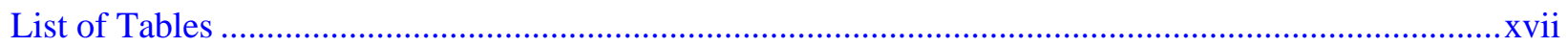

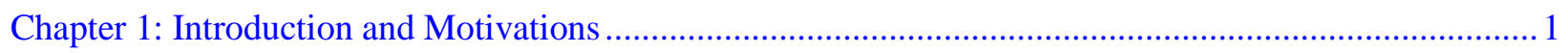

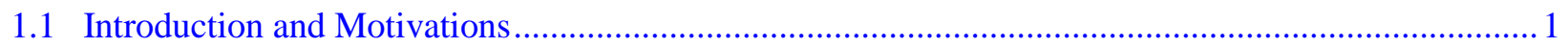

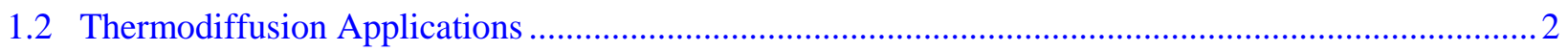

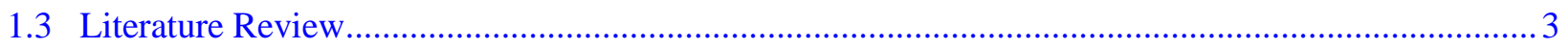

1.4 Experimental Measurement of Thermodiffusion ........................................................................ 6

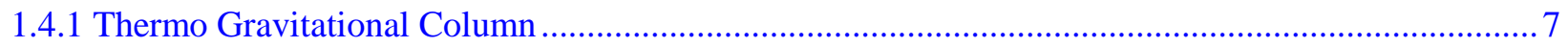

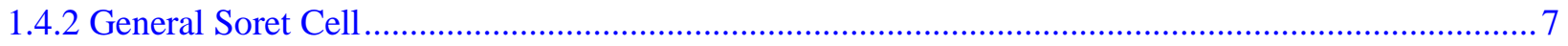

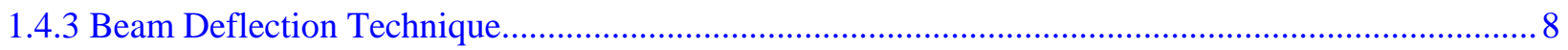

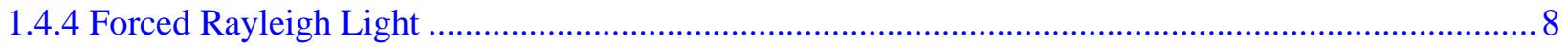

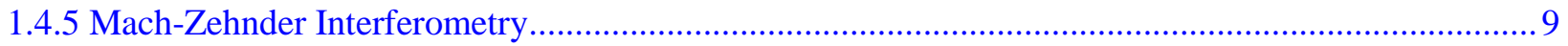

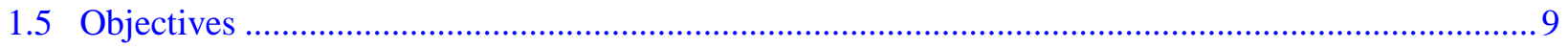

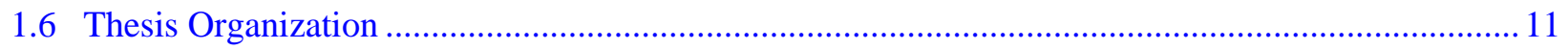

Chapter 2: Fourier Image Processing and its Application for Mach-Zehnder Interferometry ................... 13

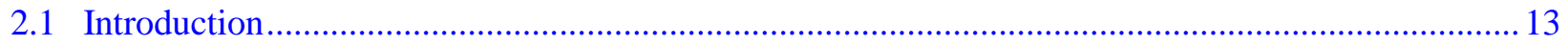

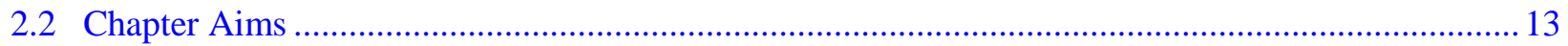

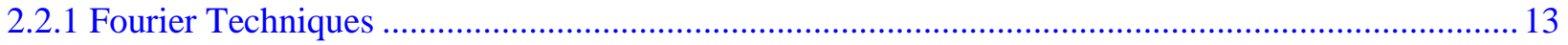




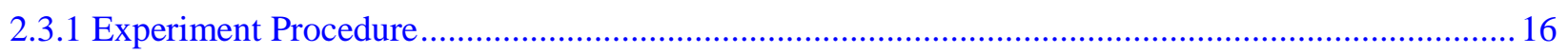

2.3.1 Software Validation for the Benchmark Binary Mixture..........................................................20

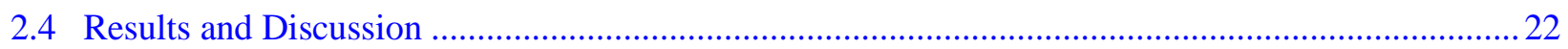

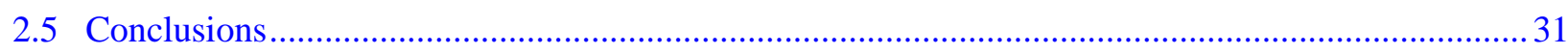

Chapter 3: Theoretical and Experimental Measurement of Thermal Time ….......................................... 33

for MZI to Study Heat and Mass Transfer Phenomena

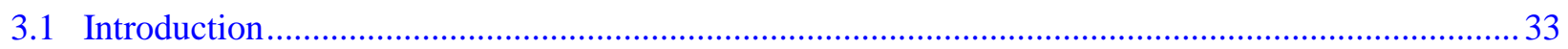

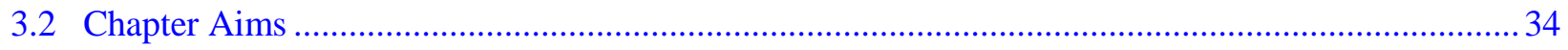

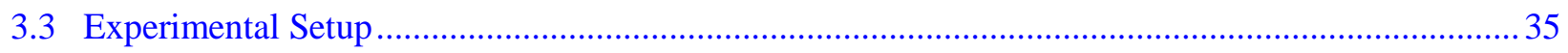

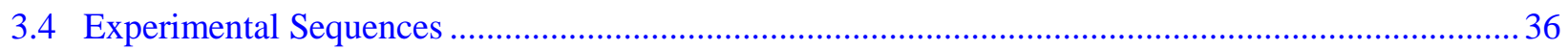

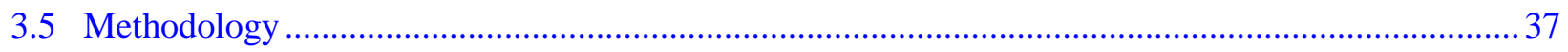

3.5.1 Solution of Energy Equation for the MZI Experiment …......................................................... 38

3.5.2 Theoretical Model to Determine Thermal Time for Mixtures with Negative Soret Coefficients ...... 40

3.5.3 Experimental Method to Determine the Thermal Time for Mixtures ............................................ 45

with a Positive Soret Coefficient

3.5.4 Calculation of $\Delta \mathrm{C}$ Correction Values for Mixtures with a Positive Soret Effect............................. 49

3.5.5 Implementation of an Iterative Evolutionary Optimization Technique to Calculate the $\mathrm{S}_{\mathrm{T}} \ldots \ldots \ldots \ldots . . .50$

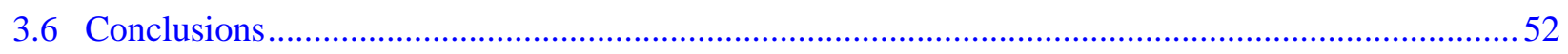

Chapter 4: Detail Heat Transfer Analysis using MZI for Two Different Cell Configurations ...................53

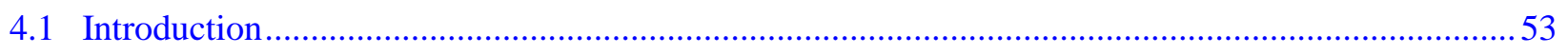

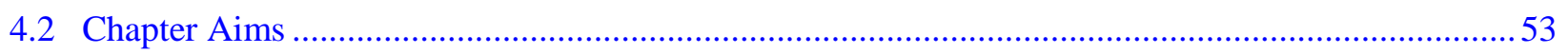

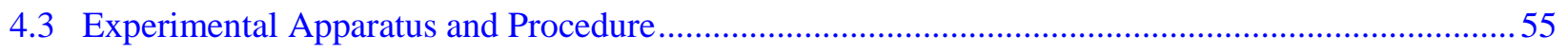

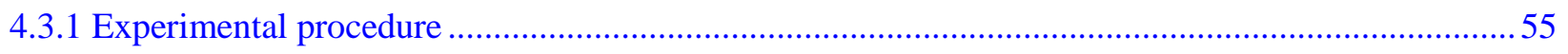

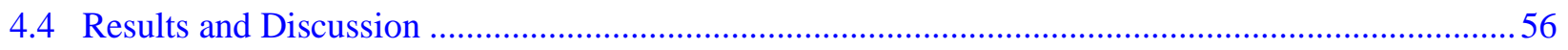




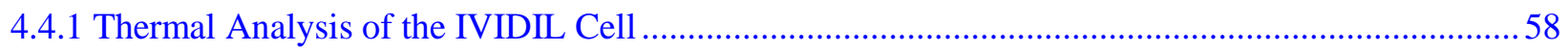

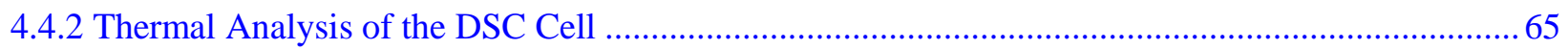

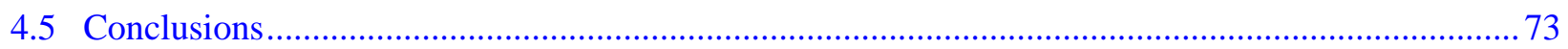

Chapter 5: Heat and Mass Transport Phenomena under the Influence of Vibration ................................75

Using a New Aided Image Processing Approach

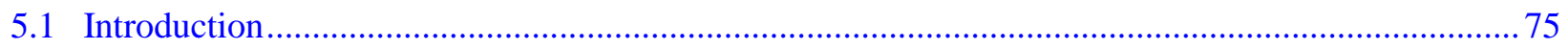

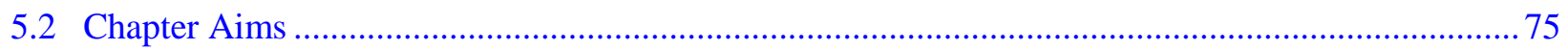

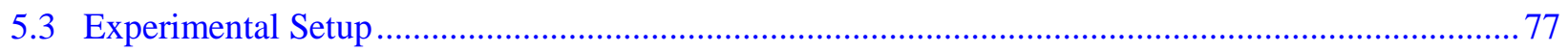

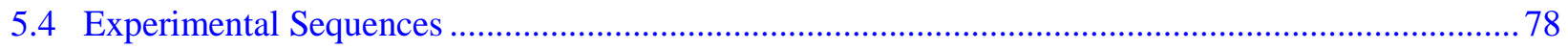

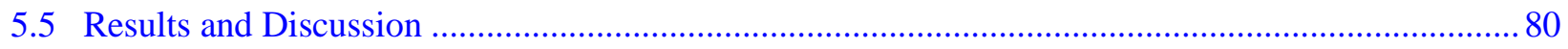

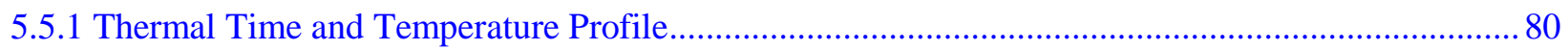

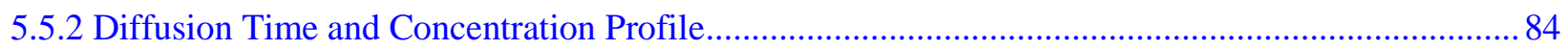

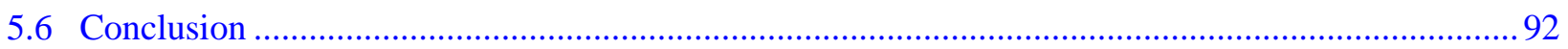

Chapter 6: Experimental Study of the Impacts of Forced Vibration on Thermodiffusion Phenomenon....94

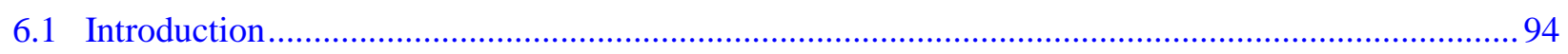

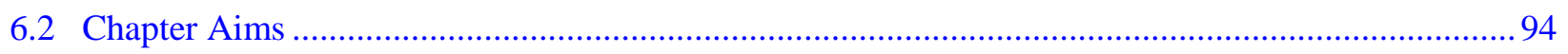

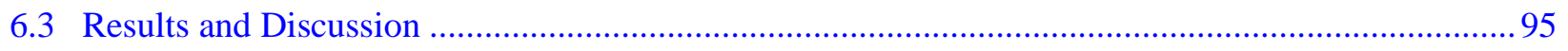

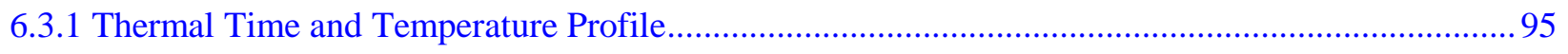

6.3.2 Diffusion Time and Concentration Profile.............................................................................. 97

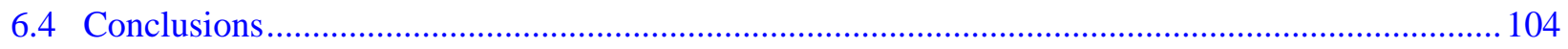

Chapter 7: New Experimental Method to Measure Pure and Cross Diffusion Coefficients...................... 105

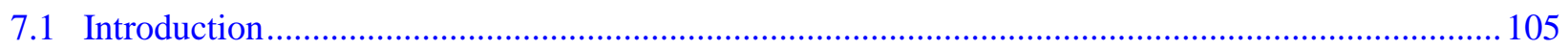

7.2 Proposed Approach to Measure Diffusion Coefficients .............................................................. 105

7.2.1 Mathematical Description of the Diffusion in a Ternary Mixture ................................................ 106

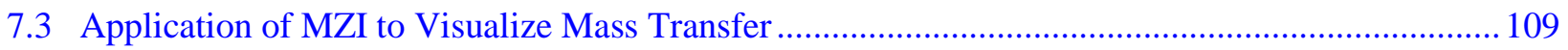




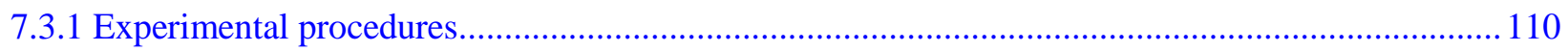

7.3.2 Advanced Image Processing Aided with an Evolutionary Algorithm ........................................ 110

7.3.3 Applications of the Proposed Method for Space Experiments .................................................... 117

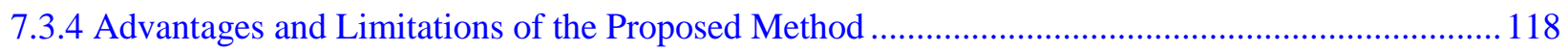

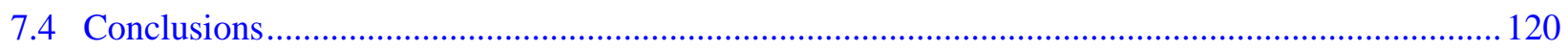

Chapter 8: Detail Analysis of DSC Experiment to Measure the Soret coefficients for ternary mixtures.121

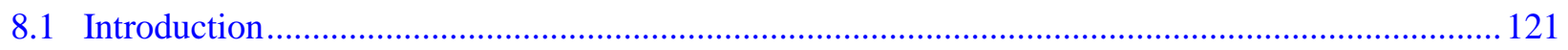

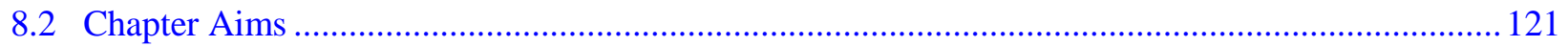

8.3 Optical Experimental Equipment for Ternary System ............................................................ 122

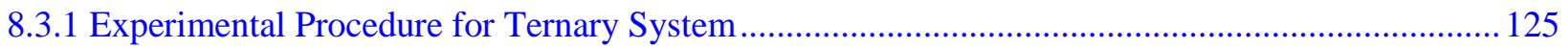

8.4 Equations Development to Study the Ternary System …........................................................ 127

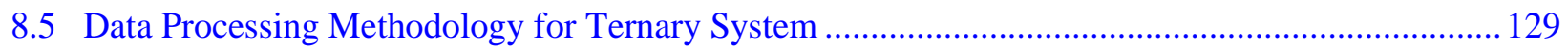

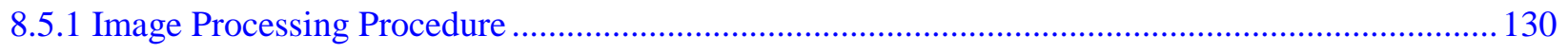

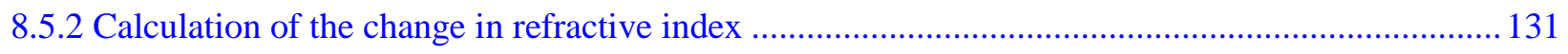

8.5.3 Experimental accuracy, calculation of temperature and concentration........................................ 132

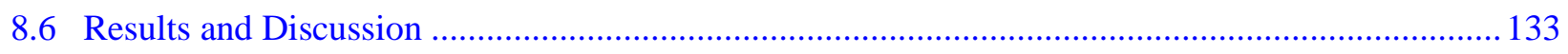

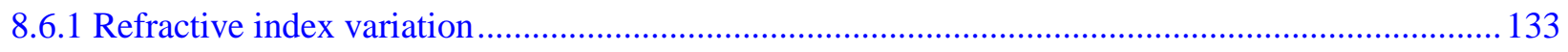

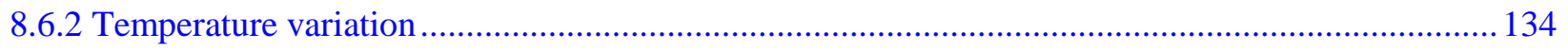

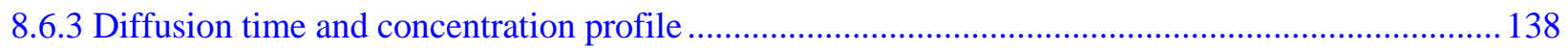

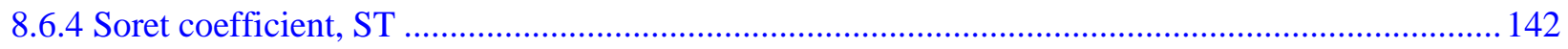

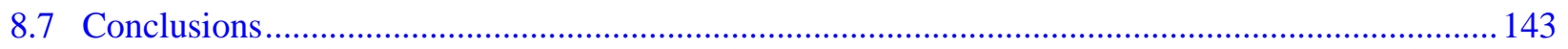

Chapter 9: Soret and Diffusion Coefficients Measurement of Ternary Mixtures of $\mathrm{C}_{12}$, IBB, THN........ 145

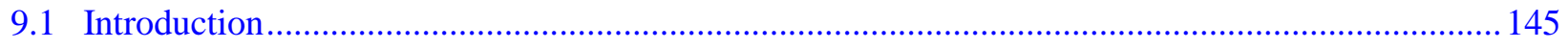

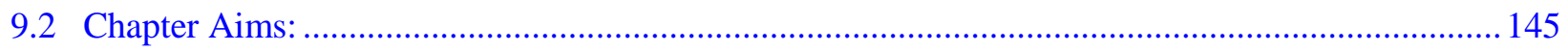

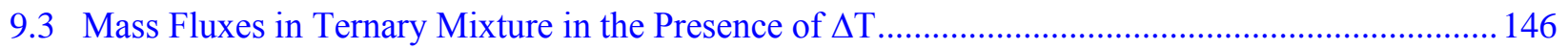




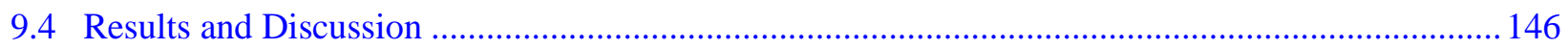

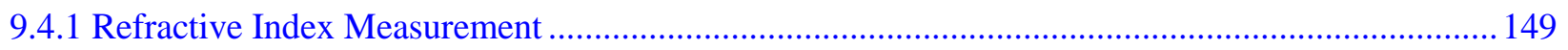

9.4.2 Linear Variation of Temperature along the Height of the Cell .................................................. 152

9.4.3 Temporal Concentration Variation During Thermodiffusion and Diffusing Phases ...................... 153

9.4.4 Soret and diffusion coefficients measurement using GA Curve Fitting Method ............................ 154

9.4.5 Separation of Non-associating Ternary Mixture along the Temperature Difference...................... 155

9.4.6 Mass Transport Coefficients of THN, IBB and C12 Mixtures ................................................. 159

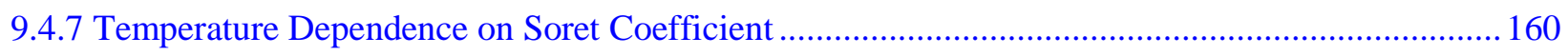

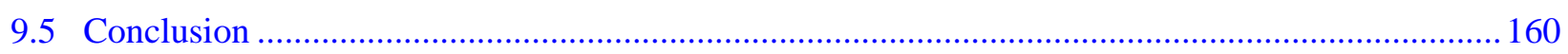

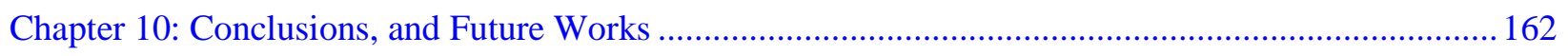

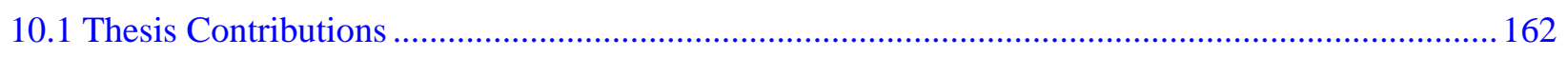

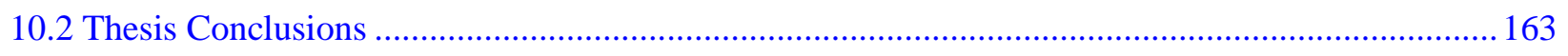

10.2.1Development of a Model to Study the Thermodiffusion using MZI .......................................... 163

10.2.2 Heat Transfer Analysis of the MZI Experiment for Space Experiment....................................... 164

10.2.3 Experimental Study on Impacts of Thermogravitational Flow on Heat and Mass Transfer.......... 165

10.2.4 Measurement of the Mass Transfer Coefficients of Binary and Ternary Mixtures ...................... 165

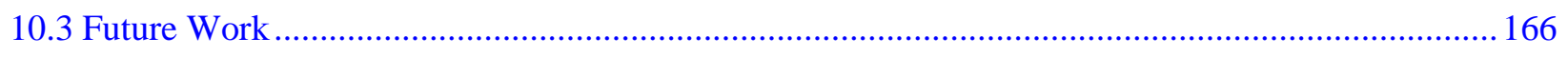

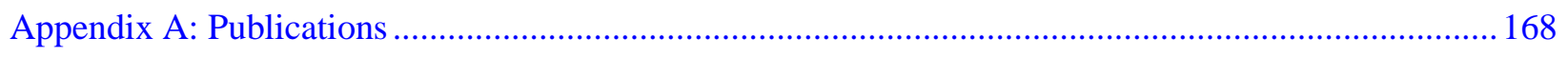

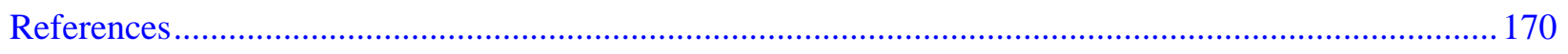




\section{Table of Figures}

Figure 1-1: General view and scheme of a thermogravitational column [73] ....................................... 6

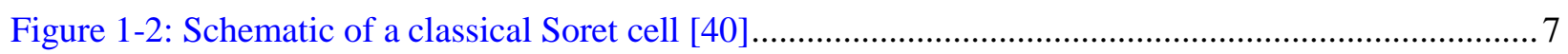

Figure 1-3: Scheme of the cross-section of a beam deflection apparatus[40,73] f................................. 8

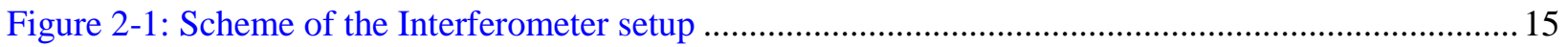

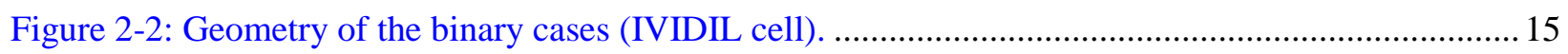

Figure 2-3: Principle scheme of Fourier image processing in this study ............................................. 17

Figure 2-4:Maximum concentration variation between hot and cold walls ...........................................24

for negative and positive Soret effect for the binary mixture

Figure 2-5: Input is a noisy fringe; output is a filtered fringe pattern,

phase map using FFT and WFF. Phase map using FFT and WFF

Figure 2-6: General results of the unwrap a phase map a) with and b)without badpixels or bad region

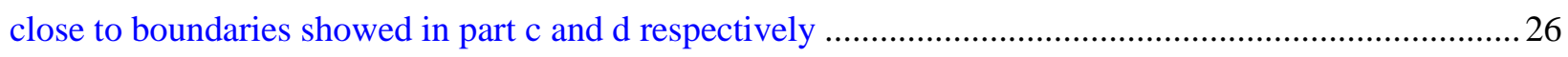

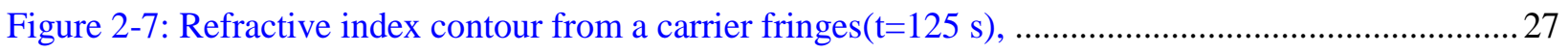

wiggly domain in evident in the first part of the figure

Figure 2-8: Temperature contours by different Fourier technique .....................................................2

after two thermal times for binary cell Run2R

Figure 2-9: IPA concentration contours in the cell for IVIDIL-Run33 ...............................................28

Figure 2-10: Results of mass fraction of IPA along different lines ....................................................2 29

Figure 2-11: Concentration variation for DSC Run 12 .................................................................... 30

at the end of thermodiffusion phase that shows the maximum separation in the system

Figure 2-12: Comparison results of FFT and WFF for a ternary case ................................................ 31

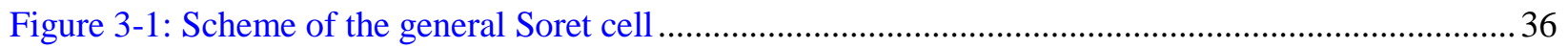


Figure 3-2: Optical digital interferometry scheme utilized

with laser means of two different wavelengths as $\lambda=670 \mathrm{~nm}$ and $\lambda=935 \mathrm{~nm}$ on-board ISS

Figure 3-3: Non dimesional Temperature variation at various locations

Figure 3-4: Temperature deviation from the linear variation

at the steady condition at different location along z-direction

Figure 3-5: Refractive index versus time during $\mathrm{L}^{2} / \chi$

Figure 3-6: Temporal maximum refractive index difference between the cold and hot walls

Figure 3-7: Temporal maximum refractive index difference

between the cold and hot walls for positive Soret effect

Figure 3-8: Temporal maximum refractive index difference

for the case with positive Soret between different locations

Figure 4-1: Geometry of the SODI cell. .56

Figure 4-2: Transient variation of mean temperature gradient normal

to the boundaries of the case 1 (IVIDIL-Run2R).

Figure 4-3: Refractive index of the front view of binary cell case 1 at (a to i) before

applying temperature gradient $0<\mathrm{t}<100 \mathrm{~s},(\mathrm{j})$ at $\mathrm{t}=220 \mathrm{~s}$ and $(\mathrm{k})$ after the thermal time $(\mathrm{t}=770 \mathrm{~s})$

Figure 4-4: Refractive index of the side view of the binary cell case 1 at (a to i before.

applying temperature gradient $0<\mathrm{t}<100 \mathrm{~s},(\mathrm{j})$ : at $\mathrm{t}=220 \mathrm{~s}$ and $(\mathrm{k})$ after the thermal time $(\mathrm{t}=770 \mathrm{~s})$

Figure 4-5: Temperature contours inside the cell during the initial isothermal stage

Figure 4-6: Temperature contours inside the cell during the initial isothermal stage

for the case 2 with the $1[\mathrm{~cm}] \mathrm{x} 1[\mathrm{~cm}] \mathrm{x} 0.5[\mathrm{~cm}](\mathrm{x}, \mathrm{y}$, and $\mathrm{z})$ cell size

Figure 4-7: Temperature variation between the hot and cold walls .68

Figure 4-8: Normal temperature gradient near boundaries of the case 1 .69

Figure 4-9: Normal temperature gradient near boundaries of the case 2 70 
Figure 4-10: Wrapped phase distribution inside the SODI cells at the end of the thermal time.

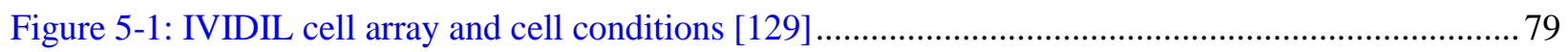

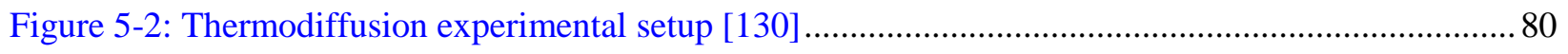

Figure 5-3: Separation versus time during entire experiment of RunExt1 ............................................ 82

Figure 5-4: Sketch of two leaser paths inside the IVIDIL cell array ................................................... 82

Figure 5-5: Temperature difference between hot and cold sides during thermal time............................. 85

Figure 5-6: Temperature variation between hot and cold walls of a) Run12 and b) Run11..................... 86

Figure 5-7: Temperature distribution in the cavity at different time during thermal time, Run12 ............87

Figure 5-8: Temperature distribution in the cavity at different time during thermal time, Run11 .............87

Figure 5-9:Separation between hot and cold sides during thermo-diffusion time ................................. 88

Figure 5-10: IPA concentration variation between hot and cold walls at various times..........................90

Figure 5-11: IPA concentration profile in the cavity at different time during diffusion time, Run12 ........91

Figure 5-12: IPA concentration profile in the cell for various runs .................................................. 92

Figure 5-13: Analytical solution and maximum experimental separation .............................................93

Figure 5-14: Soret coefficient versus Rayleigh number for all nine runs ..............................................94

Figure 6-1: Temperature variation between hot and cold walls .........................................................99

Figure 6-2: Temperature contour inside cell after thermal time …...................................................... 100

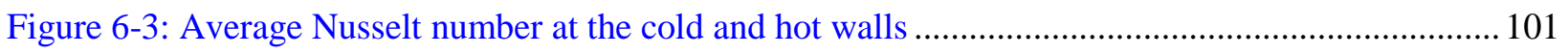

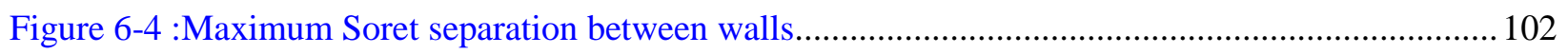

Figure 6-5:IPA concentration variation at the middle at various times ................................................ 103

Figure 6-6: IPA concentration profile inside cell for various runs after 12 hours .................................. 104

Figure 6-7 : Maximum separation of the various runs during thermodiffusion time............................... 106

Figure 6-8 Maximum separation and the time of maximum separation for all runs.............................. 107

Figure 7-1: Filteration of noise in the fringe pattern by image processing .......................................... 116 
Figure 7-2: Maximum refractive index difference between the hot and cold walls experiment.

Figure 7-3: Maximum refractive index variations of both lasers during the diffusion phase. 117

Figure 7-4: Application of robust smoothing transform on diffusion experiment. 119

Figure 7-5: Temporal concentration variation between the walls during the space experiment.

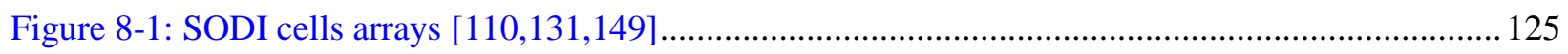

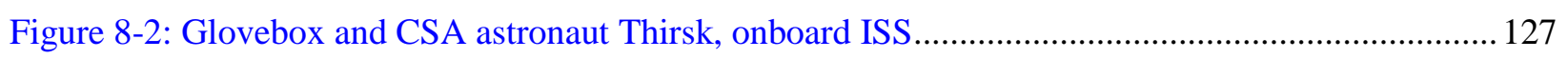

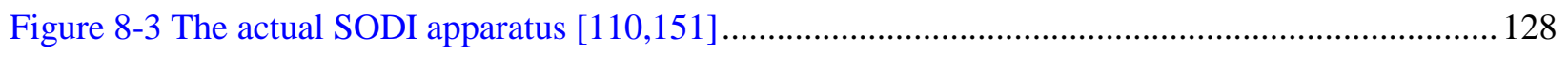

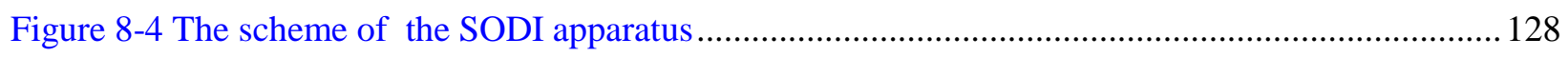

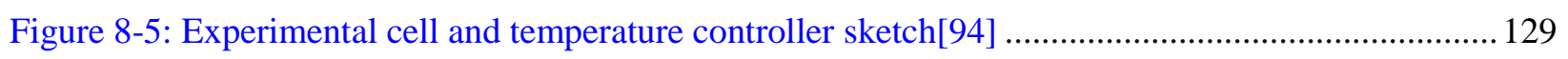

Figure 8-6: Separation versus time during thermodiffusion phase ................................................... 130

Figure 8-7: Principle scheme of Fourier Transform image processing technique to ............................ 135 analyze optical digital interferometry of a ternary system

Figure 8-8: Refractive index difference between hot and cold walls versus time 139 during isothermal, thermal and diffusion conditions

Figure 8-9:Temperature difference between hot and cold sides during thermal time 140

Figure 8-10: Temperature variation between hot and cold walls of Run7 ......................................... 141

Figure 8-11: Temperature profiles at different times during thermal time, Run12 .............................. 142

Figure 8-12: Phase and phase difference distributions in the cavity at different times ........................ 142 during thermal time, Run12

Figure 8-13: Separation between hot and cold sides during thermo-diffusion time .............................. 144

Figure 8-14: THN concentration variation between hot and cold walls at various times ....................... 144

Figure 8-15: IBB concentration variation between hot and cold walls at various times......................... 145

Figure 8-16: THN concentration at different times, Run12 ....................................................... 145

Figure 8-17: IBB concentration profiles in the cavity at different times .......................................... 146 
during diffusion time, Run12

Figure 8-18: Transient experimental separations of Run12, associated with best fitted curve. 147

Figure 9-1: Scheme of the assembled equipment

along with moving and fix bridge and the DSC cell array

Figure 9-2: General phase maps demonstrating the front view of the cell

and also the cropped region of the cell

Figure 9-3: Refractive index difference between walls during the entire experiment for cell 4 154

Figure 9-4: Refractive index difference during five different steps of the experiment 156

Figure 9-5: Temperature along the height of the cell $u$ (a) laser $\lambda=670 \mathrm{~nm}$ (b) laser $\lambda=935 \mathrm{~nm}$. 158

Figure 9-6: Concentration difference between the hot and cold sides

for two of the components versus time during thermodiffusion and diffusion phases for cell 4

Figure 9-7: Concentration difference between the hot and cold side

for two of the components versus time during thermodiffusion and diffusion phases for cell 3

Figure 9-8: Concentration difference of all three components 161

between the hot and cold sides during thermodiffusion phases for cell 2

Figure 9-9: Ternary map of mass fraction of ternary mixtures

tested onboard ISS, numbers represent the cell number 


\section{List of Tables}

Table 1: Optical properties of the binary mixture for laser with the wavelength of $670 \mathrm{~nm} \ldots \ldots \ldots \ldots \ldots \ldots . . . . . . . .18$

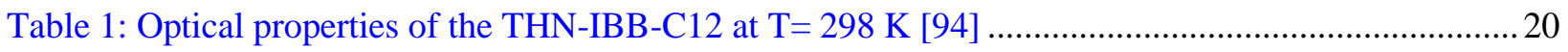

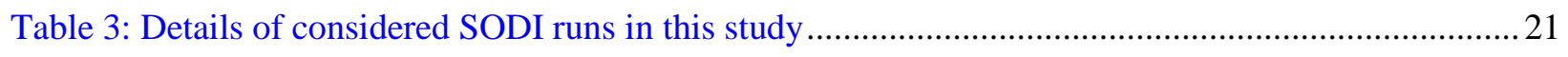

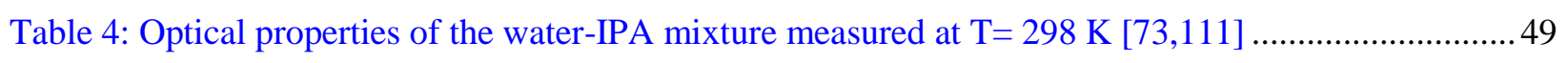

Table 5: Result of Iterative method after various iterations ............................................................51

Table 6: Summarized thermal time and Soret coefficient calculated using different .............................52

methods in comparison with assuming $\mathrm{t}_{\mathrm{th}}=\mathrm{L}^{2} / \chi$ and benchmark value of Soret coefficient

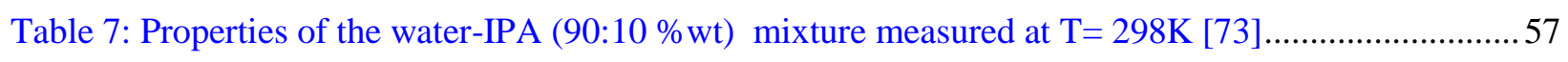

Table 8: Optical properties of the THN-IBB-C12 (10:80:10 \%wt) mixture measured [94] .....................57

Table 9: Summary of the thermal analysis results of the both IVIDIL and DSC cases ...........................74

Table 10: Comparison of $S_{T}$ using optical measured $\Delta T$ and using thermal sensor ................................ 75

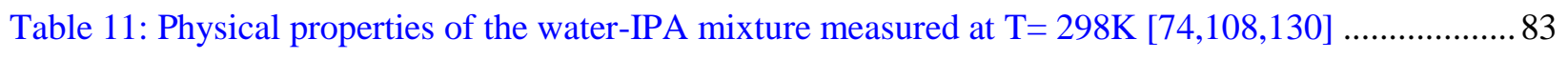

Table 12: The IVIDIL experimental runs with 10K temperature gradient onboard ISS ......................... 84

Table 13: The IVIDIL experimental results with 10K temperature gradient onboard ISS ...................... 88

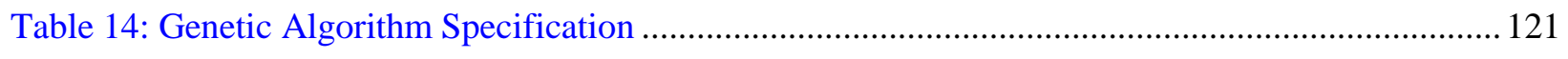

Table 15: Chemical properties of three components of the test mixtures ............................................ 129

Table 16: DSC Runs7 and 12 experimental results with 10K temperature gradient ............................. 147

Table 17: Compositions of the ternary mixture in different cells inside the cell array .......................... 153

Table 18: Detail experimental results of thermodiffusion of the ternary mixture onboard ISS ............... 163

Table 19: Mass transport coefficients of the ternary system measured ................................................ 164 


\section{Nomenclature}

\begin{tabular}{|c|c|c|c|}
\hline$A$ & Area $\left[\mathrm{m}^{2}\right]$, Amplitude $[\mathrm{m}]$ & $M_{w}$ & $\begin{array}{l}\text { molecular weight of pure } \\
\text { component }[\mathrm{g} / \mathrm{mol}]\end{array}$ \\
\hline$C$ & Fluid Concentration[-], & $P$ & Pressure $[\mathrm{Pa}]$ \\
\hline$C p$ & Specific heat $[\mathrm{J} / \mathrm{kg} \mathrm{K}]$ & $Q$ & Heat flux $\left[\mathrm{W} / \mathrm{m}^{2}\right]$ \\
\hline$D$ & Molecular diffusion coefficient $\left[\mathrm{m}^{2} / \mathrm{s}\right]$ & $R$ & Gas constant $[\mathrm{j} /(\mathrm{mol} \mathrm{K})]$ \\
\hline$D_{T}$ & Thermal diffusion coefficient $[1 / \mathrm{K}]$ & $R a$ & Ryleigh number[-] \\
\hline E & Energy $[\mathrm{J}]$ & $S_{T}$ & Soret coefficient $[1 / \mathrm{K}]$ \\
\hline Gs & Gershuni number[-] & $S_{R}$ & Soret ratio $[-]$ \\
\hline$f$ & Body force[N], Frequency $[\mathrm{Hz}]$ & $t$ & Time $[\mathrm{s}, \mathrm{min}, \mathrm{h}]$ \\
\hline$g$ & Gravitational acceleration $\left[\mathrm{m} / \mathrm{s}^{2}\right]$ & $T$ & Temperature $[\mathrm{K}]$ \\
\hline$H$ & Height of cavity [m] & $U$ & Velocity magnitude $[\mathrm{m} / \mathrm{s}]$ \\
\hline$i, j$ & Indexes & $u$ & $\begin{array}{l}\text { Velocity component in the } \mathrm{x} \\
\text { direction }[\mathrm{m} / \mathrm{s}]\end{array}$ \\
\hline$J$ & Mass flux $\left[\mathrm{kg} /\left(\mathrm{s} \mathrm{m}^{2}\right)\right]$ & $V$ & Volume $\left[\mathrm{m}^{3}\right]$ \\
\hline$k$ & Conductivity of the fluid $[\mathrm{w} /(\mathrm{m} \mathrm{K})]$ & $v$ & $\begin{array}{l}\text { Velocity component in the } y \\
\text { direction }[\mathrm{m} / \mathrm{s}]\end{array}$ \\
\hline$l$ & Length of cavity $[\mathrm{m}]$ & $x, y, z$ & Variable or axes director \\
\hline$L$ & Optical Length or path [m] & & \\
\hline$w$ & Velocity in the $\mathrm{z}$ direction $[\mathrm{m} / \mathrm{s}]$ & & \\
\hline
\end{tabular}




\section{Greek Symbols}

$\alpha, \chi \quad$ Thermal diffusivity

$\beta_{C} \quad$ Volumetric concentration expansion coefficient [-]

$\beta_{T}$

$\tau_{D}$

$\tau_{t h}$

$\mu$

$v$

$\rho$

$\omega$

$\varphi$

$\Delta \phi \quad$ Phase Distribution

$\lambda$

$\nabla$

$\Delta C$

$\Delta n$ $\left(\frac{\partial n}{\partial T}\right)$ $\left(\frac{\partial n}{\partial C}\right)$

Refractive index due concentration [-]

\section{Subscript}

Initial

Cold side

Hot side

exp Experimental

ref Reference

D Diffusion

num Numerical

th Thermal

St $\quad$ Steady

Vibration 


\section{Chapter 1: Introduction and Motivations}

\subsection{Introduction and Motivations}

Thermodiffusion, also called the Soret effect, is a phenomenon involving heat and mass transfer in a liquid, or a gas due to a temperature gradient. It is important in oil exploration and in optimal oil recovery since it causes diffusion fluxes in hydrocarbon reservoirs. Oil exploration models are being developed to simulate these phenomena over several centuries, and improved oil recovery models are also being developed to estimate and locate different compositions in oil reservoirs [1-3]. Soret coefficient values are usually ignored in most engineering applications; however, an accurate and advanced modeling of mass diffusion is critical for this kind of oil recovery investigation. This phenomenon is more pronounced in oil fields, usually due to the porous environment [4-8].

For example, in the Yufutsu oil field in Japan, Ghorayeb and his team have conducted a set of pressure, fluid composition, and temperature measurements in a gas field to explore the unusual fluid distribution[8-10]. The data recorded along different vertical wells showed that there exists a motionless liquid in the middle and two lighter vapor section at the top and the bottom of the well. Further analysis indicated that thermodiffusion played the main role in displacing the fluid and moving gas below the fluid surface [8-10]. Hence, a precise and better understanding of thermodiffusion phenomena in multi-component mixtures would result in a more accurate modeling of oil reservoirs, and it may reduce the number of required wells, which costs approximately $\$ 20$ million/well. Additionally, thermodiffusion can have applications in environment discovery [11], microfluidic applications [12,13], particle motion in microfluidic devices[14], and in the field of DNA studies[15-17].

While the Soret effect in binary mixtures is well-studied[18-24]; only a few literature reports are dedicated to the thermodiffusion phenomenon in ternary or quaternary mixtures [25-28]. The Soret coefficient is recognized as difficult to determine at ground conditions because of technical constraints in the control of liquid convection and convective instabilities for most of the mixtures [29-33]. The fluid properties measurements conducted in reduced or microgravity environments minimize the influences of gravity. A microgravity environment suggests a 
suitable location for studying the behavior of liquid mixtures and measuring the diffusion and thermodiffusion coefficients. Here, the Soret effect in multicomponent mixtures was analyzed using the results of the experiment conducted in a microgravity environment.

\subsection{Thermodiffusion Applications}

Enhancement in experimental techniques along with importance of thermodiffusion in petroleum industry has caused noticeable increase of the research in this phenomenon. Thermodiffusion has applications in different important processes in technology and nature. However, its main critical is for oil extraction from the hydrocarbon reservoirs, that are subjected to noticeable temperature gradients. Thus, thermodiffusion or Soret effect is being investigated by considerable amount of research in various systems such as liquid hydrocarbons, polymers, gases, electrolytes, proteins, combustion, surfactant micelles, latex particles, alcohols and Ferro fluids [34-38]; while, most of the works have been devoted to the petroleum industry. These industries complete huge amount of research to understand the separation of the components under Soret effect and then to determine the fluid distribution in the oil reservoirs [30,36,39,40]. Thermodiffusion is also has role in isotropic separation of liquids, molecule manipulation by temperature gradient thermophoresis, etc. Moreover, recently it is found that Soret effects in nanofluids can change the concentration variation from a homogenous condition more than twenty percent [41]. Finally, thermodiffusion plays significant role in emerging applications such as particle manipulation by temperature gradient for microfluidic applications, and in optical screening methods for biomolecules and colloids $[42,43]$.

In the underground oil reservoirs, the geothermal temperature difference coupled with the stationary conditions of the liquid that causes separation process in the porous layer of soil and sand. Generally, there are various forces contributing to distribution of fluid such as gravity, thermal gradient and other fluxes. It is an important challenge to consider all these phenomena to measure the connectivity of the different panels and layers in an oil field [30,44-46]. It is believed that the reverse geothermal gradient, through thermodiffusion and the gravity field, through gravitational segregation, are the two important factor that govern the concentration distribution of the fluid in the large oil reservoirs [39]. 
The most precise method of determining the thermodiffusion coefficients is by performing experiments. Nevertheless, obtaining accurate experimental data out of any experimental method is challenging. Because of the magnitudes of these coefficients that are smaller than $10^{-9}$, an extensive accurate experimental method must be used that is expensive in most of the cases. On the other hand the molecular-dynamics simulation can provide qualitative or semi quantitative data to study the thermodiffusion phenomenon [28,47-50].

\subsection{Literature Review}

When the composition of a mixture is non-uniform, the concentration difference in any component of the mixture presents a driving potential force that causes diffusion of that species. As a result of this diffusion, any specific species flows from regions with the higher concentration to regions with the lower concentration. The Maxwell-Stefan theory is a proposed equation that describes diffusion in multicomponent mixtures.

This model that represents the mass fluxes in the mixture has been proposed by James Clerk Maxwell for dilute gases and Josef Stefan for fluids [51,52]. It is completely similar to the diffusion of heat from regions of high temperature to regions of low temperature. The Fick's diffusion theory is the simplified form of the Maxwell-Stefan equation and it does not exclude the possibility of negative diffusion coefficients [53]. Finally, it must be mentioned that the effective diffusivity is one for ideal mixtures.

Different theoretical models and investigations are developed by Eslamian et al [28,49,54,55] and Abbasi et al [56-58] to study the Soret effect. One of the main factors in estimating the thermodiffusion coefficient for associating and non-associating fluid mixtures is evaluation of the activation energy in Eyring's viscosity theory [59]. They applied several methods to estimate the activation energies of pure components, and then extended them to complex hydrocarbon mixtures. The kinetics approach and phenomenological approach are two different approaches in modeling thermodiffusion coefficients. The kinetics approach is based on the irreversible thermodynamics theory and thermodiffusion coefficients are based on the specific heat of transport of the two components in a binary mixture. 
Demirel and Sandler [60] developed the linear-non-equilibrium thermodynamics theory for coupled heat and mass transport at the beginning of the 20th century. It observes that the analysis of linear-nonequilibrium thermodynamics defines the correct forces and flows involved in an irreversible process because the entropy production depends on the flows and forces. Linearnonequilibrium thermodynamics (LNET) was used to present dissipation functions and the entropy generation.

Tai and Char surveyed the effects of thermal diffusion on heat and mass transfer by free convection flow [61], the laminar free convection flow in a presence of thermal radiation and Soret phenomena along a vertical plate within a porous medium was studied. The influence of Soret and Dufour diffusion and thermal radiation and power-law fluid index was applied in this study. In the results, temperature and concentration fields and Sherwood number for various cases of the controlling parameters were discussed.

Different sources such as experiment operation, aerodynamic drag, gravity gradients, life-support systems, equipment operation, crew activities, and rotational effects create microgravity vibrations on board vehicles in microgravity environments [5,62-64]. This microgravity vibration is one of the most important factors that can affect thermodiffusion experiments on board the ISS. Other factors are errors in the optical setup, errors in the measurements, and analysis errors. It has been argued that controlled vibrations or g-jitter vibrations in a reduced gravity condition can reduce the separation of components in a mixture [65-68]. For this reason, the IVIDIL (Influence of Vibrations on Diffusion of Liquids) experiment investigated the effect of vibrations on heat and mass transfer phenomena and Sore effect.

The effects of low frequency G-jitter on the thermal diffusion are studied by Yan et al [32]. The mixture water-isopropanol with ten percent mass fraction of isopropanol bounded in a cubic cell was simulated with a lateral heating and different vibration conditions. In earlier studies, it had been approved that low frequency vibrations show a more dangerous consequence on diffusion measurements with respect to high frequency vibrations.

The numerical study indicated that both residual gravity and g-jitter might be detrimental but also beneficial to achieve purely diffusive conditions. The g-jitter was found to reduce compositional variation $[63,64,69]$. They assumed a single-phase fluid and all physical 
properties, except the fluid density, as constant values with the assigned values obtained at mean temperature; however, according to the investigations by Saghir and Seshasai [62,70], this assumption is not accurate enough. In their method, the time integration was performed using a first-order implicit scheme. In the case of zero gravity, the density variation was very smooth. In presence of residual gravity, the density increased to a maximum value situated at around 3/4 of the cavity length, and then decreased to an intermediate value at the cold wall. The influence of the frequency of the g-jitter Soret effect in investigated [37].

The effects of Soret and Dufour on free convection along a vertical wavy surface were investigated by Narayana et al [71,72]. Free convection caused by heat and mass transfer along with a wavy surface in a Newtonian, saturated, porous medium was surveyed by applying cross Dufour and Soret diffusion in the medium. Under the large Darcy-Rayleigh number, assumption a similarity solution to this problem was presented.

It is observed that a single convective cell in all the mixtures caused by a steady static microgravity in all two dimensions was formed [35]. A stronger velocity perpendicular to the direction of the thermal gradient was observed.

Impact of the vibrations on Soret separation in binary and ternary mixtures is investigated [36]. In this survey, they simulated two ternary hydrocarbon mixtures and one binary associating. One of the ternary mixtures was assumed a pressure of $35 \mathrm{MPa}$ and the other mixtures were at the atmospheric pressure. The vibrations were recorded by the accelerometers onboard the international space station. Based on their previous model, the thermal gradient was applied in the horizontal direction of a two-dimensional domain. At first, they simulated environment corresponding to an ideal zero gravity or purely diffusive scenario, and after that they focused on case, the demeaned Root Mean Squared (RMS) values of the acceleration. Their assumption was a constant thermal conductivity and no internal energy generation [36]. They stated that performing diffusion experiment for liquid mixtures on the ISS was not reliable.

The results of analysis showed that unlike the purely diffusive scenario, imposing the ISS vibrations had a profound effect on the Soret effect in all three systems. More precisely, in all three mixtures, a single convective cell was observed to make a mixing effect; this phenomenon destroyed most of the Soret separation. The effect of vibrations to remove the Soret effect on the 
low-pressure mixture was more than high-pressure mixture. In addition, this happened as a result of the fact that in lower pressure the fluids respond occur more easily to the applied vibrations. The double-diffusive thermal convection with the Soret effect process presented to different vibration conditions is simulated in a zero gravity condition [73].

The thermal gradient is applied to cavity perpendicular to the vibration. The thermal properties of the fluid as well as the concentration profiles were scrutinized on a long time scale, during thermal time. The system was subjected to the three different levels of Rayleigh vibrations by using different frequencies, amplitudes, and temperature gradients. The results showed that using variable properties from PC-SAFT make the outcomes more reasonable in comparison with the model with constant properties. It is observed that a strong mixing was found in cases with high Rayleigh vibrations.

\subsection{Experimental Measurement of Thermodiffusion}

There are several experimental techniques to investigate the Soret effect, such as the thermogravitational column [74-77], the diffusion cell [78], thermal flow field fractionation [79], light scattering [18,80,81], thermodiffusion-forced Rayleigh Scattering [81-84], laser beam deflection [26,85], and holographic interferometry [23,86-88].

A group of experimental techniques involves classical measurements to achieve large separation ratios. Classical Soret cell, thermogravitational column, the two-chamber thermodiffusion cell, and thermal field-flow fractionation are part of this group [22,74,75,77,89,90].

Another group of experimental approaches is intended to establish stable thermal and mechanical conditions to prevent convection process by employing optical techniques. The laser-beam deflection technique, thermal diffusion forced Rayleigh scattering, thermal lens technique, and the microfluidic fluorescence methods are all optical approaches used in laboratories on earth; while, they are able to be used in microgravity environments. A review of the experimental techniques to measure the Soret coefficient was performed by Srinivasan and Saghir [35]. 


\subsubsection{Thermo Gravitational Column}

A Thermo gravitational column (TGC) consists of two vertical plates maintained at two different temperatures that creates horizontal temperature gradient. Generally, height of the column is less than a meter and there is a 1 or $2 \mathrm{~mm}$ gap between the plates.
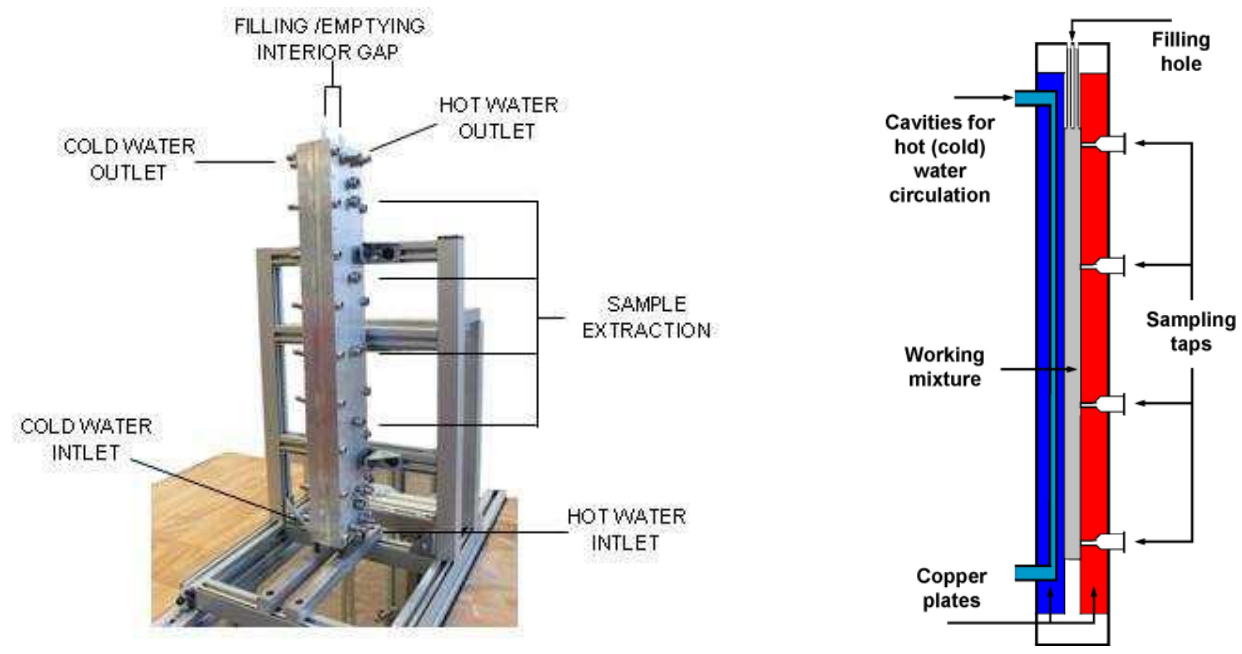

Figure 1-1: General view and scheme of a thermogravitational column [75]

The upper side of the apparatus consists of two small holes for filling of the column and for air to escape during the filling [91]. The temperature gradient is applied by increasing the temperature of one of the sidewalls. Consequently, Soret effect causes each component move to different sides, which causes the lateral concentration gradient. Then the sample of the liquid can be extracted to find the Soret coefficients.

\subsubsection{General Soret Cell}

A general Soret cell is constructed with two horizontal plane plates. These plates are normally made of stainless steel or copper because of heat conduction capabilities. There is a gap between these plates and the temperature gradient is proposed across the plates. There are also some holes drilled on top and bottom of the plates. During the experiment, to have transient variation of concentration, small portions of samples are collected (closed to the isothermal horizontal boundaries) [92] with a needle and a watertight device that exist in each hole. 


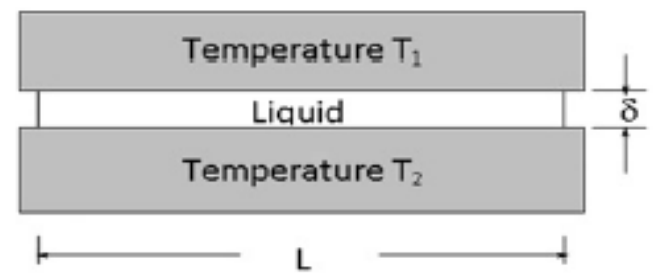

Figure 1-2: Schematic of a classical Soret cell [35]

\subsubsection{Beam Deflection Technique}

There is another advanced form of Soret cell that the concept of beam deflection techniques is used to measure the concentration of the sample. This method is one of the optical approaches to measure the Soret effect. The main modification in beam deflection technique is that two opposite transparent lateral walls are assigned and for the lateral walls, the glass is used because it allows obtaining the change of index of refraction along the beam path that is parallel with the plates. When there is no thermal or concentration gradient, a laser beam would not reflect from the horizontal path. On the other hand, any gradient of concentration or temperature cause the laser beam not to propagate horizontally.
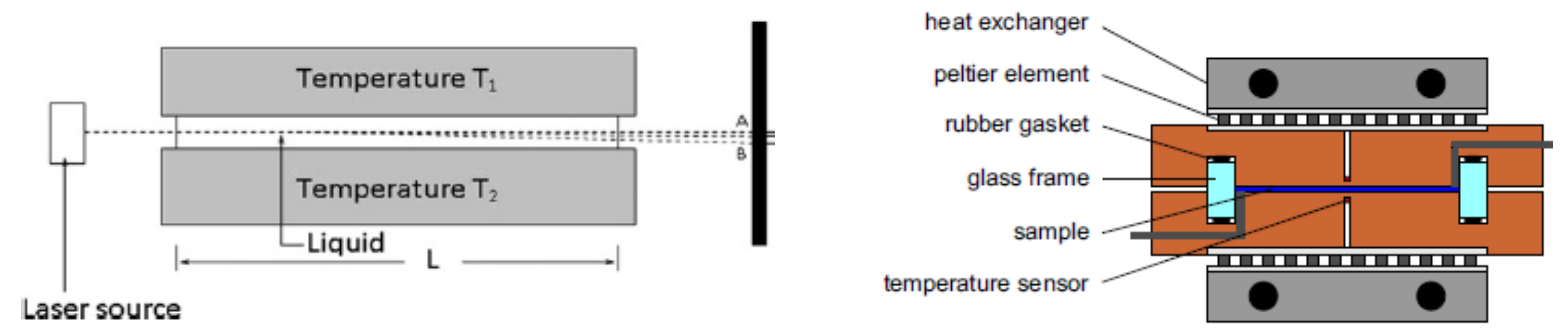

Figure 1-3: Scheme of the cross-section of a beam deflection apparatus. "A and B are the initial and steady state points of incidence of the laser beam on the screen" [35,75]

More detail of the beam deflection method to measure of thermodiffusion in liquid mixtures is described in the literature [93]. It is worth noting that the Soret coefficient of thermodiffusion experiments on polystyrene colloids with special functionality in water has been measured using the same technique [93].

\subsubsection{Forced Rayleigh Light}

The forced Rayleigh light scattering method provides a useful and sensitive instrument for the thermodiffusion studies. This method is to study thermal diffusive behavior of mixtures with 
holographic grading technique. In this method, a laser beam is divided into two beams of equal density. These two beams were converged at the experimental sample. An intensity gradient can be measured using these two lasers in the transparent mixture and also the temperature and the concentration gradients cause a refractive index gradient in the mixture, which can be obtained with the diffraction of a third laser beam $[35,49]$.

\subsubsection{Mach-Zehnder Interferometry}

Another technique, which is used here, is Mach-Zehnder interferometry (MZI) that is an optical method. The detail information of MZI is provided in this thesis based on its application for for heat transfer analysis or thermodiffusion analysis in binary or ternary mixtures.

Most of the above mentioned experimental techniques and the development of theoretical models have been mainly performed on binary mixtures [94]. This has caused a gap of ternary and multicomponent experimental results that has stopped the enhancement of the current multicomponent thermodiffusion models. However, it should be noted that there are few investigations that can be addressed for studying the Soret effect in multicomponent mixtures $[26,28,50,95]$. So here, in addition to studying the thermodiffusion in binary mixtures, the MZI equipped with two lasers is used to study the thermodiffusion in ternary mixtures as well.

In this thesis, the accuracy of the MZI method that was used to measure the mass transport coefficients for the binary mixture has been investigated. Then, the Soret coefficients of benchmark binary and ternary mixtures were obtained using the experimental results sent from the ISS. In addition to that, the effects of the forced vibrations on the thermodiffusion process were analyzed.

\subsection{Objectives}

The main objective of this study is to investigate the thermodiffusion phenomenon in the multicomponent mixtures. In order to achieve this objective, two series of thermodiffusion experiments conducted on board the International Space Station were analyzed and the sub objectives of this research were: 
1) To investigate the impacts, technical difficulties, advantages and disadvantages of applying 2D Fourier fringe analysis and phase unwrapping, with particular for the experimental results of Mach-Zehnder interferometry. Although there is wide range of applications for this method, it was used in this thesis to analyze the thermodiffusion experiments and to implement the twodimensional Fourier fringe analysis and phase unwrapping for interferometry-based thermodiffusion experiments according to the literature;

2) To implement the Windowed Fourier Transformation (WFT) method and Windowed Fourier Filtering (WFF), Genetic Algorithm (GA), and fast robust smoothing function to analyze the thermodiffusion process using Mach-Zehnder Interferometry.

3) To design and develop a robust methodology to estimate the Soret and diffusion coefficients. The methodology should be able to measure the Soret coefficient for both binary and ternary mixtures in the presence of G-jitter or force vibrations.

4) To determine the most accurate value for thermal time (especially in situation when heat and mass transfer are coupled). The experimental thermal time for the MZI experiment has a direct effect on the estimation of the Soret coefficients because of the high ratio of the separation at the beginning of the experiment. So accurate determination of the thermal time is an essential condition to have accurate measurement of the mass transfer coefficients.

5) To study the effect of forced vibrations on the thermodiffusion experiments using the results of the IVIDIL experiment. Acceleration or gravity disturbs the pure thermodiffusion pattern and because there are G-jitter vibrations onboard ISS; the effect of vibration on the thermodiffusion experiment must be determined and the critical condition should be determined in which the pure separation does not occur.

6) And to analyze the thermal performance of the SODI cell and also to measure the Soret coefficients of a benchmark hydrocarbon mixture at various compositions using the experimental results obtained on board the ISS. The performance of the thermal unit of the SODI apparatus must be analyzed to ensure the linear variation of the temperature variation across the cell was achieved during the thermodiffusion experiment. 


\subsection{Thesis Organization}

This thesis consists of the following eight chapters:

In chapter 2, the application of the windowed Fourier transformation to analyze the heat and mass transfer problem using the MZI setup is explained and discussed. A detailed comparison between FFT and WFT results for binary and ternary mixtures of the MZI thermodiffusion experiments is provided. The substantial enhancements of the method are presented and discussed for different experiments conducted for both binary and ternary mixtures. The importance of replacing the FFT method becomes more pronounced for the ternary system, as this method fails to reconcile reliable concentration profiles.

In chapter 3, a theoretical approach and experimental models are employed to estimate the thermal relaxation time of the optical digital interferometry experiment that deals with the coupling between the heat and mass transfer phenomena. Mach-Zehnder interferometry (MZI) was found to be an accurate and precise experimental model to visualize the thermodiffusion phenomena inside the cubic cell when a thermal gradient is applied at two sides of the cell.

In chapter 4, the thermal performance of the SODI apparatus is extensively analyzed. The results obtained from the binary mixture (IVIDIL) and the ternary case (DSC) are presented separately and then are compared together. The heat transfer and temperature measurements during the thermodiffusion experiments at reduced gravity were investigated. The sensitivity of the temperature measurements using interferometry was examined, and the effects the weak insulation at the walls was investigated on the Soret separation.

In Chapter 5 and 6, the possible influences of a wide range of forced vibrations on thermodiffusion measurements in the condition of microgravity subjected to two temperature differences were investigated. However, results indicate a maximum separation and Soret coefficient for the case with a minimum Gershuni number, while different concentration profiles and separation patterns were observed. These various behaviors are discussed in detail in this chapter.

Chapter 7 reports a new experimental method to measure diffusion coefficients of ternary mixture using MZI equipped with two lasers; while, the diffusion and the thermodiffusion 
coefficients of binary mixtures have been measured and analyzed in detail in chapters 5 and 6 . An evolutionary algorithm known as genetic algorithm is used to find an accurate estimation of the transport coefficients.

Chapter 8 provides detail experimental information of heat and mass transfer analysis of the SODI experiment for a ternary mixture at specific composition. It reports the quality of the DSC experiment for measurement of mass transport coefficients of ternary mixture.

Chapter 9, which is mainly dedicated to the entire results of the DSC experiment, reports the separation in a ternary hydrocarbon mixture of tetrahydronaphtalene, isobutylbenzene, and dodecane due to a thermal gradient. Also, the Soret and diffusion coefficients of this mixture at five different compositions were measured. The reliability and the repeatability of the MZI experiments to study thermodiffusion for ternary mixtures were investigated. Finally, the mass diffusion in various compositions of this mixture was compared, and the effect of mean temperature on the Soret effect was studied.

In the final chapter (Chapter 10), the conclusions of this research are described. In addition to that, some explanations about the contributions of this thesis and the future work are provided. 


\section{Chapter 2: Fourier Image Processing and its Application for Mach-Zehnder Interferometry}

\subsection{Introduction}

Transform techniques arrange for analyzing and processing signals in a domain, the most appropriate and efficient form of this transform is required operations for image processing. There are many different transform techniques employed for image processing; however, these techniques all have limitations and no method is suitable for all types of problems [96]. There are continuous research efforts into expanding, adapting, and improving the available transform techniques for different applications of signal processing such as audio or image processing. A two-dimensional domain such as image or 2D audio representation allows the application of transform techniques in two dimensions. The use of 2D transform techniques for image processing has been investigated and it may produce interesting and useful results [97-101].

\subsection{Chapter Aims}

This chapter mainly explains the application of 2D transform techniques for image processing of digital optical interferometry, particularly on the use of two different 2D Fourier transform techniques (FFT and WFT). It also aims to discover how information can be gained about an image by analyzing as a $2 \mathrm{D}$ array using the $2 \mathrm{D}$ Fourier transform and to investigate which methods of transform data processing can be used to provide the more precise result out of a MZI image [96].

In order to achieve these aims a large element of application development is required during the investigation. The main objective of this chapter will be to produce a software tool that enables analysis and transformation of images into the different 2D Fourier transform domain.

\subsubsection{Fourier Techniques}

In the late 19th century, Joseph Fourier proposed a theorem that any periodic signal can be decomposed into a series of harmonically sinusoidal functions with specified amplitude, frequency, and phase offset; this is known as the Fourier series [102-104]. The Fourier transform 
uses this concept for decomposition of a continuous-time signal into sinusoidal components. Fourier analysis is extremely important in many areas of signal processing [105]. Equations 2-1 and 2-2 represent the Fourier and inverse Fourier transforms respectively for any continue function.

$$
\begin{aligned}
& G(u)=\int_{-\infty}^{\infty} g(x) e^{-j 2 \pi u x} d x \\
& g(x)=\int_{-\infty}^{\infty} G(u) e^{j 2 \pi u x} d u
\end{aligned}
$$

where the independent variable $\mathrm{x}$ represents time, the transform variable $\mathrm{u}$ represents frequency.

\subsubsection{Fast Fourier transform}

It was found that the Discrete Fourier transform (DFT) was not efficient enough to be used in the all signal processing because it requires both complexes multiply and add operations. The fast Fourier transform, introduced in the 1960s [106,107]. This improved efficiency has allowed the application of the Fourier transform in many signal-processing tasks and real-time Fourier processing would be possible. There are many different implementations of the FFT available; however, MATLAB has built in functions, which employ the FFT algorithm[108].

\subsection{Fringe Pattern Analysis for SODI facilities onboard ISS}

The Soret coefficient, which is a materials property, is recognized to be difficult to determine at ground conditions because of technical constraints in the control of liquid convection and convective instabilities due to gravity [63,94]. Measurements conducted in controlled microgravity environments such as the International Space Station (ISS) minimize the perturbation effects of gravity and allow purely diffusive conditions to be achieved. The best example of such measurements is the experiments conducted with the SODI facilities (Selectable Optical Diagnostics Instrument). The two major detailed experiments that have been performed under the SODI facility are IVIDIL and DSC. The IVIDIL experiment investigated the effect of vibrations on the thermal separation of binary mixtures $[64,66,94,109,110]$. The main purpose of DSC(Diffusion Soret Coefficient) was to measure the diffusion and Soret coefficients of selected ternary mixtures, a process that is fundamentally more complex and sensitive than the 
measurements on binary mixtures [111]. The outcome of these experiments can be employed to test thermodiffusion theories and develop physical and mathematical models for the estimation of thermodiffusion and diffusion coefficients [110,112]. Two binary mixtures of waterisopropanol (IPA) and the ternary mixture of tetrahydronaphthalene-isobutylbenzene-dodecane (THN-IBB-C $\mathrm{C}_{12}$ ) with various compositions were hosted in the cell arrays for the binary cases (IVIDIL) and the ternary cases (DSC) experiments, respectively.

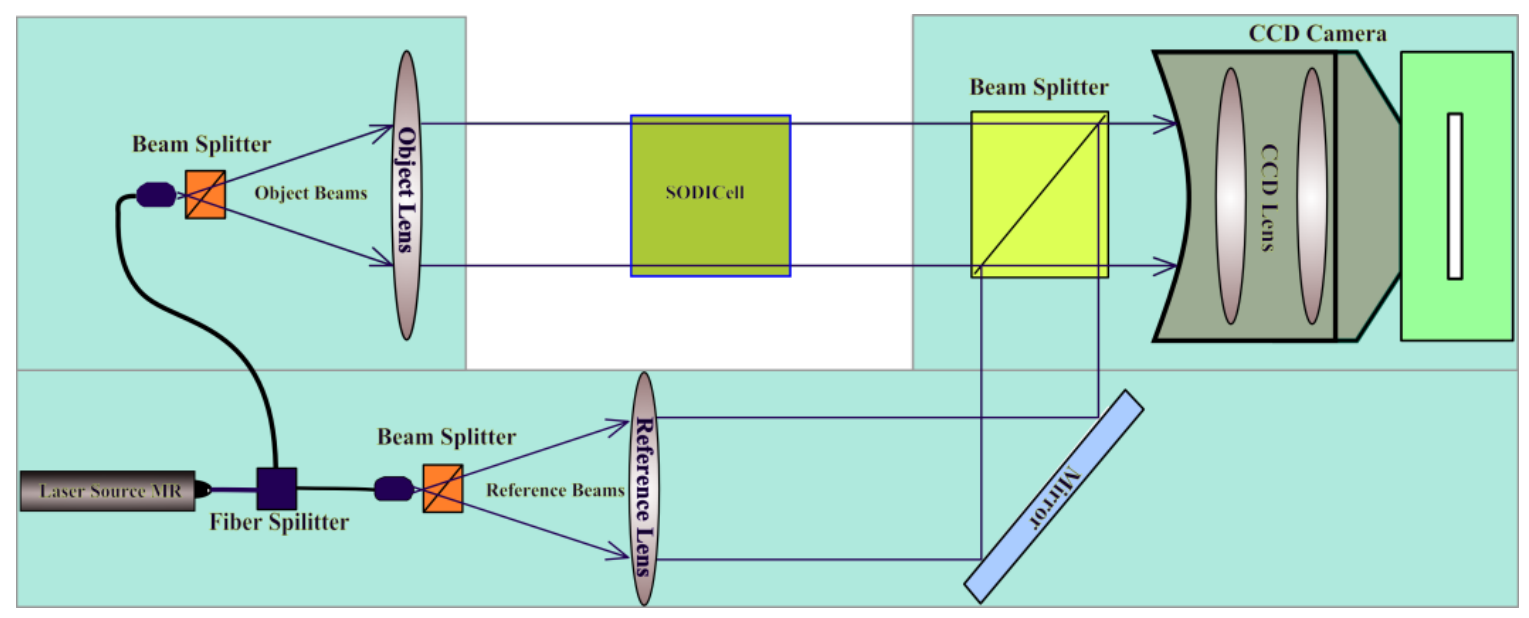

Figure 2-1: Scheme of the Interferometer setup, it represents the moving bridge of SODI apparatus

The experimental data for both of these experiments were obtained using the interferometry image processing technique $[110,112]$, which is one of the non-contact optical methods for these systems.

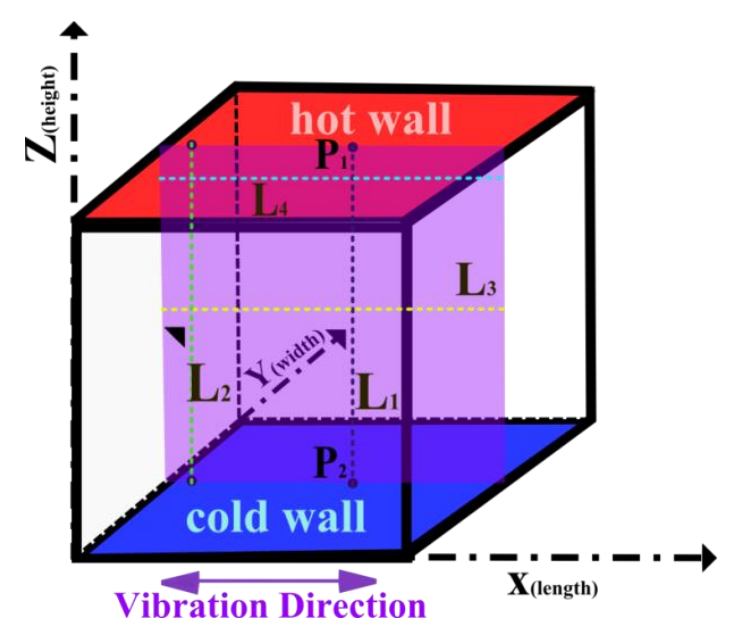

Figure 2-2: Geometry of the binary cases (IVIDIL cell). 
The samples were monitored by a Mach-Zehnder interferometer at two wavelengths. Because of small scale of the variations in concentration, the processed results can be affected by the inherent noise that exists in the system due to many reasons such as presence of dust particles on the front glass disturbing a clear view of the cell. Here, for the first time we have implemented a windowed Fourier transform algorithm to study the thermodiffusion phenomenon. The results and discussion are provided in latter Section, and they focus on a detailed comparison between the FFT and WFT results in studying the binary and ternary MZI experiments. Finally, a summary of this work is provided in the conclusion section.

\subsubsection{Experiment Procedure}

Fringe analysis techniques in which are effective, reliable, and non-contact methods for measuring refractive index variation [98,103,113], have been employed in both the DSC and the IVIDIL experiments. As shown in the Figure 2-1, the generated laser beam was propagated into an optical fiber cable. The sample was monitored using a Mach-Zehnder interferometer at two wavelengths, depending on whether the sample was a binary or ternary mixture. Figure 2-1 shows the two different paths inside the cell holder of the split beam. One of the paths led the beam through the front of the cell (in the direction perpendicular to the thermal gradient, as shown in Figure 2-2) and the other one passed the beam through the void area to be used as the reference beam.

The first step of the thermodiffusion experiments that will be analyzed in this section was thermalization of the sample at the mean temperature to reach the initial state of uniform concentration and temperature distribution inside the cavity. At this stage, the top and bottom plates of the cell were maintained at the mean temperature. Then, the temperature gradient was built up by application of heat to both sides of the cavity. MZI images and the corresponding temperature differential between the hot and cold sides were acquired during the experiment. After completion of the designated experimental time span, the temperature difference was removed and the cell was brought back to the mean temperature. If needed, the run was repeated at a different temperature difference. Afterwards, the moveable optic was relocated to another sample and the cycle was repeated. 
Thermal equilibrium in the sample mixture is established at a characteristic time that is a function of the length of the experimental cell and the thermal diffusivity of the liquid mixture: $\tau_{t h} \propto L^{2} / \chi$
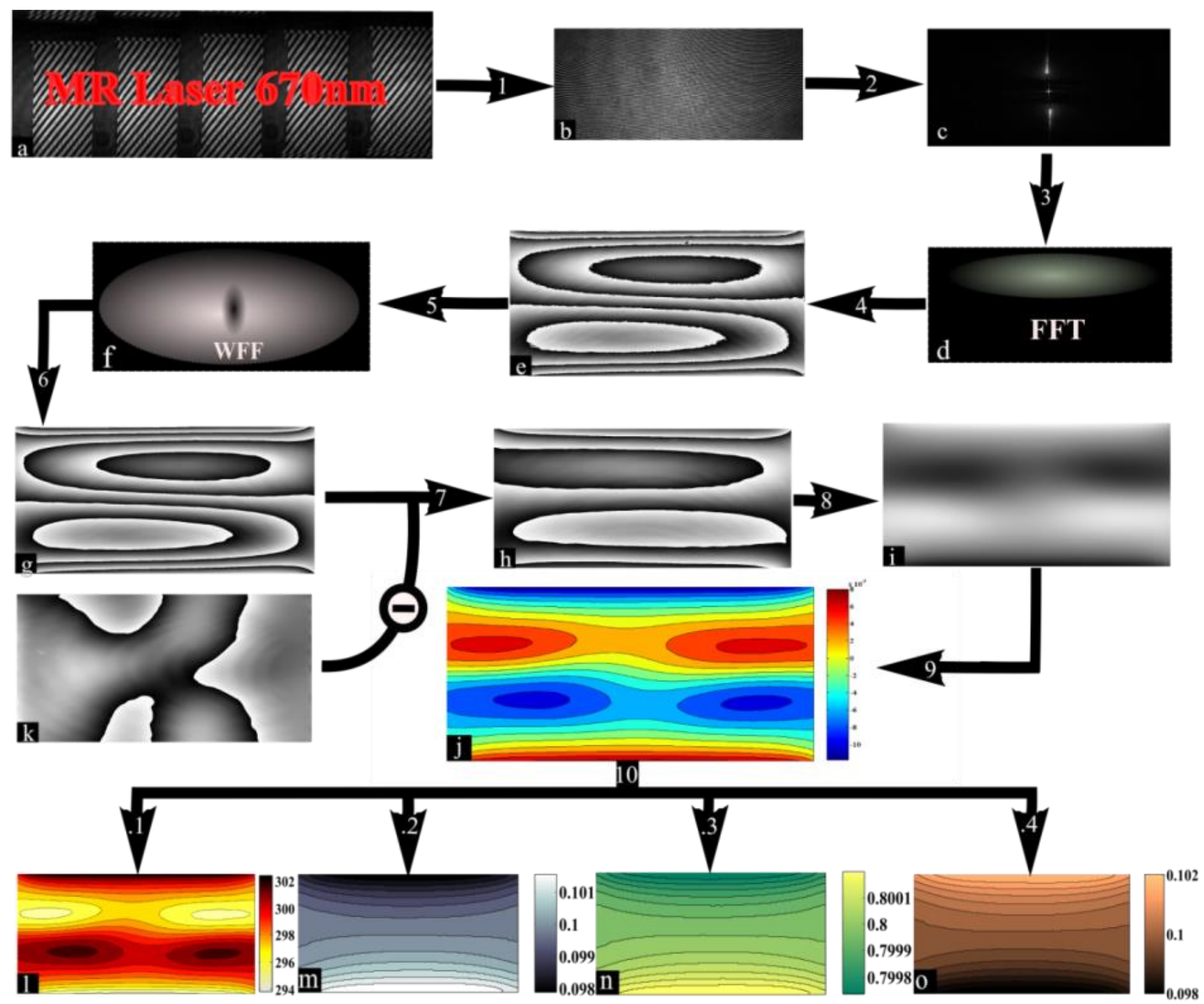

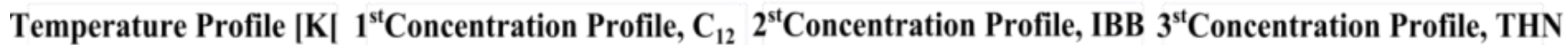

Figure 2-3: Principle scheme of Fourier image processing in this study (1: Converting and Cropping, 2: Fast Fourier Transform, 3: Filter out the non-zero peaks using band filter, 4: Inverse 2D FFT,5: Windowed Fourier Filter, 6: Inverse WFT, 7: Subtracting the image from the reference image(k), 8: Unwrapping, 9:Calculationg of Refractive index, and 10: Concentration and Temperature)

It can be assumed that any change in the refractive index is caused by temperature variation during the first thermal time. In addition, there is a characteristic diffusion time that is similar to 
the thermal time as it is also a function of the cell length: $\tau_{C} \propto L^{2} / D$, where D is the smallest eigenvalue of the diffusion matrix. By definition, at the end of the diffusion time the linear separation of species has been reached [112]. In general, the thermal time is about 100 times smaller than the diffusion time. As a result, the temperature field reaches the steady state a few minutes after applying the temperature difference, after which time the changes in the refractive index are purely due to the variation in species concentration.

It must be noted that the refractive index or index of refraction is an optical non-dimensional number, which represents the ratio of the speed of light in vacuum over the speed of light in a transparent medium. The diffusive mass transport in a multicomponent mixture in the presence of a temperature gradient is described by more than two fluxes: one as a result of the temperature difference and other independent mass fluxes. There is no need to define the last mass flux for the $i^{\text {th }}$ component because the final closing relationship describes the last mass flux completely. The mass fluxes along the direction of the thermal gradient are given:

$\vec{J}_{i}=-\rho\left(\sum_{j=1}^{n-1} D_{i j} \nabla c+D_{T, i} c_{o}\left(1-c_{0}\right) \nabla T\right)$

where $\rho$ represents the density of the mixture, $c_{0}$ denotes the mass fraction of the $i^{\text {th }}$ component, $D_{i i}$ is the pure diffusion coefficient with $\mathrm{i} \neq \mathrm{j}$ for the cross diffusion coefficients in porous media, and $D_{T, i}$ represents the thermodiffusion coefficients of the components in the ternary mixture.

Table 1: Optical properties of the water-IPA mixture measured at $\mathrm{T}=298 \mathrm{~K}$ for laser with the wavelength of 670 $\mathrm{nm}(\mathrm{MR})$ and isopropanol/water cases [75,112]

\begin{tabular}{|c|c|c|c|c|c|}
\hline $\begin{array}{c}c_{o, I P A} \\
{[\mathrm{wt}-]}\end{array}$ & $\begin{array}{c}\left(\frac{\partial n}{\partial T}\right)_{p . c} \\
\times 10^{-4}\left[\mathrm{~K}^{-1}\right]\end{array}$ & $\begin{array}{c}\left(\frac{\partial n}{\partial c}\right)_{p . T} \\
\times 10^{-2}[-]\end{array}$ & $\begin{array}{c}S_{T} \times 10^{-3} \\
{\left[K^{-1}\right]}\end{array}$ & $\begin{array}{c}D \times 10^{-10} \\
{\left[\mathrm{~m}^{2} \mathrm{~s}^{-1}\right]}\end{array}$ & $\begin{array}{c}D_{T} \times 10^{-13} \\
{\left[\mathrm{~m}^{2} \mathrm{~s}^{-1} \mathrm{~K}^{-1}\right]}\end{array}$ \\
\hline $10 \%$ & -1.3427 & -9.23 & $-8.47 \pm 1.5$ & $7.11 \pm 0.4$ & $-60.2 \pm 10$ \\
\hline $50 \%$ & -3.474 & -3.64 & $5.68 \pm 0.3$ & $1.60 \pm 0.1$ & $9.57 \pm 0.5$ \\
\hline
\end{tabular}

The MZI is set up to test thermodiffusion phenomena in multicomponent mixtures that include $\mathrm{n}$ various components. This process theoretically requires $\mathrm{n}-1$ laser sources with different wavelengths. According to interferometry principles, each laser can provide one equation, as below, that has n-1 unknowns during the diffusion time: $\Delta C_{i}$ where $\mathrm{i}=1,2, \ldots, \mathrm{n}-1$. For a binary mixture and in a convectionless regime the analytical transient solution is given by equation 2-4: 


$$
\Delta C(t)=C(t, L)-C(t, 0)=-S_{T} C_{0}\left(1-C_{0}\right) \Delta T\left[1-\frac{8}{\pi^{2}} \sum_{n, \text { odd }}^{\infty} \frac{1}{n^{2}} \exp \left(-n^{2} \frac{t}{\tau_{D}}\right)\right]
$$

In the above equation, $S_{\mathrm{T}}$ (Soret coefficient) and $\tau_{\mathrm{D}}$ (a function of mass diffusion coefficient) are unknown coefficients that were calculated using the temporal concentration difference between hot and cold sides of the cell. Here the above equation was used for validation using the benchmark value of $S_{\mathrm{T}}$ and $\tau_{\mathrm{D}}$ coefficients for water/IPA mixtures.

In addition, the refractive index variation in the MZI experiment can be formulated as;

$$
\begin{aligned}
& \Delta n_{i}(x, y)=\left(\frac{\partial n_{i}}{\partial T}\right)_{T_{0}, \lambda_{i}} \Delta T(x, y)+\sum_{j=1}^{n-1}\left(\left(\frac{\partial n_{i}}{\partial c_{j}}\right)_{T_{0}, \lambda_{i}} \Delta C_{j}(x, y)\right) \\
& \Delta T(x, y)=\frac{\Delta n_{i}(x, y)}{\left(\frac{\partial n_{i}}{\partial T}\right)_{T_{0}, C_{0}, \lambda_{i}}}
\end{aligned}
$$

where $\Delta T(x, y)$ and $\Delta C_{i}(x, y)$ are respectively the temperature and concentration changes in an arbitrary point $(\mathrm{x}, \mathrm{y}) ; \frac{\partial n}{\partial T}$ and $\frac{\partial n}{\partial c}$ are known contrast factors defined as the variation of the refractive index due to temperature and concentration, respectively, while keeping the other parameter constant.

The contrast factors are functions of composition and temperature; however, due to the small variations of these parameters in the current study, these coefficients are assumed constant for each mixture. The values for the contrast factors are adopted from reference [95,112] for the mean temperature of the mixtures $\left(25^{\circ} \mathrm{C}\right)$ for binary and ternary mixtures.

Because thermal time was noticeably smaller than diffusion time, ignoring a few minutes at the beginning of the experiment will not affect the final concentration profile at the end of diffusion time. Consequently, during the first thermal time equation 2-5 can be rewritten as follows:

According to the theory of heat transfer in a liquid system, the temperature profile will not change after the first thermal time. Consequently, after that time, any derivative of temperature is equal to zero and equation 2-5 can be rewritten as follows when there are two lasers for a ternary mixture: 


$$
\begin{aligned}
& \Delta n_{1}(x, y)=\left(\frac{\partial n_{1}}{\partial c_{1}}\right)_{T_{0}, C_{2}, \lambda_{4}} \Delta C_{1}(x, y)+\left(\frac{\partial n_{1}}{\partial c_{2}}\right)_{T_{0}, C_{1}, \lambda_{4}} \Delta C_{2}(x, y) \\
& \Delta n_{2}(x, y)=\left(\frac{\partial n_{2}}{\partial c_{1}}\right)_{T_{0}, C_{2}, \lambda_{2}} \Delta C_{1}(x, y)+\left(\frac{\partial n_{2}}{\partial c_{2}}\right)_{T_{0}, C_{1}, \lambda_{2}} \Delta C_{2}(x, y)
\end{aligned}
$$

So, the mass fraction of the first two components can be calculated as follows in the next page:

$$
\left(\begin{array}{l}
\Delta C_{1}(x, y) \\
\Delta C_{2}(x, y)
\end{array}\right)=\left(\begin{array}{ll}
\left(\frac{\partial n_{1}}{\partial c_{1}}\right)_{T_{0}, C_{2}, \lambda_{1}} & \left(\frac{\partial n_{1}}{\partial c_{2}}\right)_{T_{0}, C_{1}, \lambda_{1}} \\
\left(\frac{\partial n_{2}}{\partial c_{1}}\right)_{T_{0}, C_{2}, \lambda_{2}} & \left(\frac{\partial n_{2}}{\partial c_{2}}\right)_{T_{0}, C 1, \lambda_{2}}
\end{array}\right)^{-1}\left(\begin{array}{l}
\Delta n_{1}(x, y) \\
\Delta n_{2}(x, y)
\end{array}\right)
$$

The concentration of the third component can be found based on species mass conservation [27]. The entire image processing procedure of the current work is illustrated in Figure 2-3.

Table 2: Optical properties of the THN-IBB-C12 (10:80:10\%wt) mixture measured at T=298 K for case 2 DSC case, Case12 [95]

\begin{tabular}{|c|c|c|c|c|c|}
\hline $\begin{array}{c}\text { Laser } \\
\text { Type }\end{array}$ & $\begin{array}{c}\lambda \\
{[\mathrm{nm}]}\end{array}$ & $\begin{array}{c}(\partial n / \partial T)_{p, I B B, T H N} \\
/ 10^{-4}\left[\mathrm{~K}^{-1}\right]\end{array}$ & $\begin{array}{c}\left(\partial n / \partial c_{C_{12}}\right)_{p, T, T H N} \\
/ 10^{-2}[-]\end{array}$ & $\begin{array}{c}\left(\partial n / \partial c_{T H N}\right)_{p, I B B} \\
/ 10^{-1}[-]\end{array}$ & $\begin{array}{c}\left(\partial n / \partial c_{I B B}\right)_{p, T, C_{12}} \\
/ 10^{-2}[-]\end{array}$ \\
\hline MR & 670 & -4.812 & -5.72 & 1.213 & -7.57 \\
\hline MN & 935 & -4.763 & -5.38 & 1.240 & -6.96 \\
\hline
\end{tabular}

The main difference between using this new method (WFT) and the simple FFT is the implementation of steps 5 and 6 that represent the windowed Fourier filtration technique. The addition of these two steps increases the total processing time by a factor of 40 . Nevertheless, the outcome of the analysis justifies the importance of using this method for such MZI experiments that deal with simultaneous heat and mass transfer.

\subsubsection{Software Validation for the Benchmark Binary Mixture}


In order to process the experimental the temperature and concentration contrast factors of the binary and ternary mixture are required as provided in Table 1 and Table 2 for the binary and the ternary mixtures respectively. In total, six runs of the binary and ternary cases (the IVIDIL and DSC experiments) were analyzed in this study (see Table 3): one run with a forced vibration and positive Soret coefficient, and the other five without an external vibration. Four of IVIDIL runs with no forced vibration were considered in this section, and they have been selected herein for validation.

Table 3: Details of considered SODI runs in this study

\begin{tabular}{|c|c|c|c|c|c|c|}
\hline SODI Run \# & C0,IPA & $\mathrm{f}[\mathrm{Hz}]$ & $\mathrm{A}[\mathrm{mm}]$ & $\Delta \mathrm{T}[\mathrm{K}]$ & $\begin{array}{c}\mathrm{S}_{\mathrm{T}} \times 10^{3}\left[\mathrm{~K}^{-1}\right] \\
\text { (This study) }\end{array}$ & $\begin{array}{c}\mathrm{Dx}^{10} 0^{10}\left[\mathrm{~m}^{2} \mathrm{~s}^{-1}\right] \\
\text { (This study) }\end{array}$ \\
\hline IVIDIL Run 2 & $10 \%$ & 0 & 0 & 5 & -8.81 & 6.59 \\
\hline IVIDIL Run 2R & $10 \%$ & 0 & 0 & 5 & -8.58 & 6.85 \\
\hline IVIDIL Run 33 & $50 \%$ & 0 & 0 & 15 & 5.16 & 1.61 \\
\hline IVIDIL Run 33R & $50 \%$ & 0 & 0 & 15 & 4.96 & 1.60 \\
\hline IVIDIL Run 39 & $50 \%$ & 0.05 & 70 & 10 & --- & - \\
\hline Ternary case & $80 \% \mathrm{IBB}, 10 \%$ THN, \\
DSC Run12 & --- & & & & - \\
\hline
\end{tabular}

Run 2 and Run2R were used for comparison to runs with negative Soret coefficients with $10 \%$ initial mass fractions of IPA and a $5 \mathrm{~K}$ temperature differential; in addition, Run 33 and Run33R were included and possess (50\% initial IPA mass fraction) positive Soret coefficients and a $\Delta \mathrm{T}$ $=15 \mathrm{~K}$. The 'R' suffix in the title of the runs instants for "repeated".

In order to validate the software for the different conditions of the Soret effect, two major benchmark cases with positive or negative Soret coefficients were chosen to prove the accuracy of the software for stable and unstable conditions of Soret.

The temporal separation of the isopropanol is shown in Figure 2-4 for runs 2, 2R, 33, and 33R. The maximum separation was calculated based on the average concentration of the 100 pixels along the $\mathrm{x}$-direction and close to points $\mathrm{P}_{1}$ and $\mathrm{P}_{2}$ in Figure 2-2 Moreover, the best-fit curves to the runs with positive and negative Soret coefficients are plotted in this Figure. The first 12 hours illustrates the thermodiffusion separation, while the last 6 hours is the diffusion time of the experiment. It must be noted that, $\left(\partial n_{2} / \partial c_{1}\right)_{p_{0}, T_{0}, C_{3}, \nu_{2}}$ means the rate of change of the refractive index with a change in the concentration of the first component in the condition of initial pressure, temperature, and constant mass fraction of the third component while the second laser is being used. 
The results show the reproducibility of the system as the repeated runs are fit closely. The curve of the separation rate is fairly smooth as a result of using the accurate image processing method (WFT). Moreover, the results are in agreement with the theoretical trends obtained by using the benchmark Soret and diffusion coefficients. For a quantitative comparison, the Soret and diffusion coefficients were determined for each experimental run based on the concentration difference presented in Figure 2-4. All of the measured coefficients are within the error band of the benchmark studies and the relative error is maintained at less than $8 \%$ with respect to the mean benchmark values of the diffusion coefficients.

\subsection{Results and Discussion}

In this section, the results obtained by windowed Fourier transform (WFT) for a typical MZI thermodiffusion experiment were compared with the basic Fourier transforms or phase shifting methods. Several parameters, such as the refractive index, temperatures, and concentration fields, for the binary and ternary mixtures were investigated. The concentration and temperature profiles were captured on a plane at the middle of the cavity as shown in Figure 2-2. The variation of the considered parameters along lines $\mathrm{L}_{1}, \mathrm{~L}_{2}, \mathrm{~L}_{3}$, and $\mathrm{L}_{4}$ at the middle and close to the walls on this plane are studied. Finally, the separation between points $\mathrm{P}_{1}$ and $\mathrm{P}_{2}$ was also used for comparison purposes.

Many studies have used the FFT method to analyze the images obtained by the MZI technique for thermodiffusion experiments, such as the extensive works of Shevtsova et al. $[65,66,109,114]$. The results obtained by this method usually contain a noisy concentration (or refractive index) field, especially at the interface of the contour bands. These massive fluctuations are not related to the physical characteristics of the system, such as the size of the cavity, but rather are a consequence of the image processing technique (see Figure 2-5).

The wrapped phase map by FFT is illustrated in Figure 2-6a and the corresponding unwrapped image is shown in Figure 2-6c. Performing the unwrapping procedure with a wiggly boundary causes all neighboring pixels to be affected by the noise. Despite this, the wrapped phase filtered by WFF becomes clear and smooth, as shown in Figure 2-6d. The corresponding plot using FFT is shown in Figure 2-6c. In this case, the strongest spot that exists in the fringe images may cause 
5 or 25 percent error in the calculation of the refractive index of Figure 2-5b, respectively, because it combines two-phase bands together during the unwrapping process.

The refractive index can be calculated from the unwrapped phase map. However, when FFT was used, fluctuations in the refractive index contours were created, as shown in Figure 2-7a, especially close to the hot and cold walls. The pattern obtained by the WFF method (Figure 2-7b), however, was smooth and all of the local noise and fluctuations were removed. These observations were also obtained for the temperature profile calculated. A linear temperature variation was attained in the cavity at the end of the thermal time $(\mathrm{t}=12 \mathrm{~h})$ at the middle of the cell.

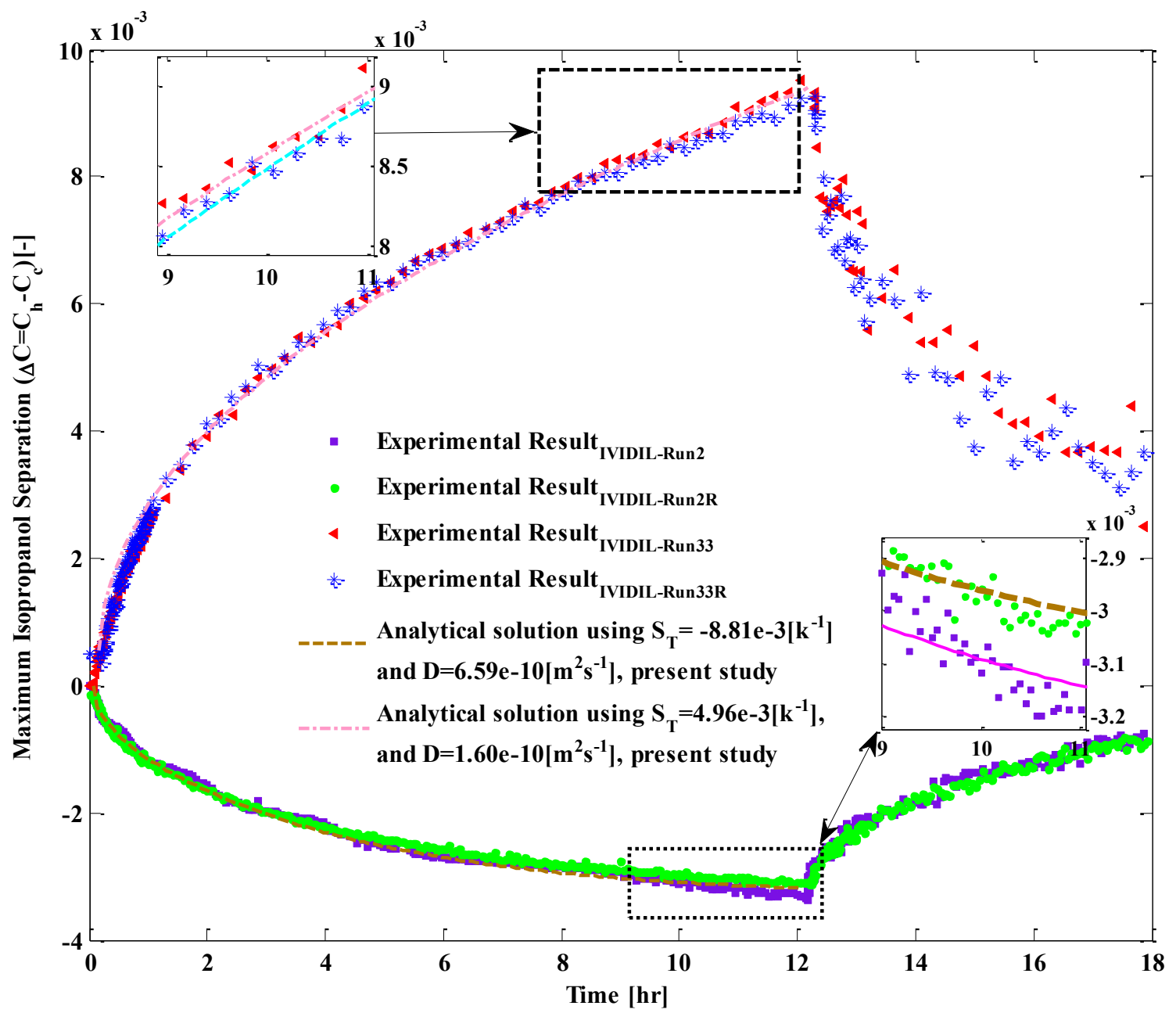

Figure 2-4:Maximum concentration variation between hot and cold walls for negative and positive Soret effect for the binary mixture 
Figure 2-8 displays the contours of the temperature field inside the binary cell during Run33. The obtained temperature field was much smoother for the case of WFT (Figure 2-8b) over that of the FFT method (Figure 2-8a). WFF provided the exact and precise temperature profile inside the cell; however, the FFT measurements were also acceptable for the temperature field and the errors were locally limited. Thus, the FFT method was reliable in measuring the temperature in the range of the current study, that is, with a $5 \mathrm{~K}$ temperature difference in the system. The nonlinearity in the temperature variation observed at the corners was due to a small lateral gap between the quartz glass and both the cold and hot walls of the cell. The same observation was recorded in other studies[112].

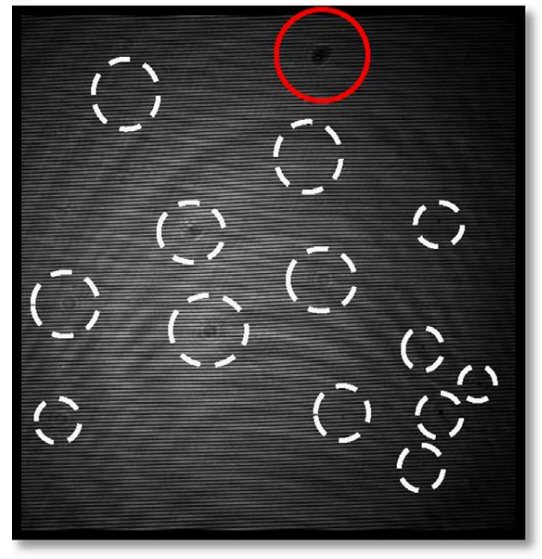

(a) carrier fringe patter

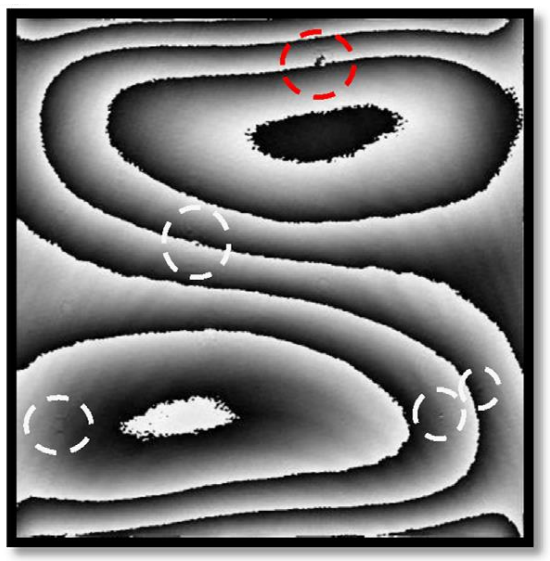

(b) phase by FFT at $\mathrm{t}=12.05 \mathrm{~h}$

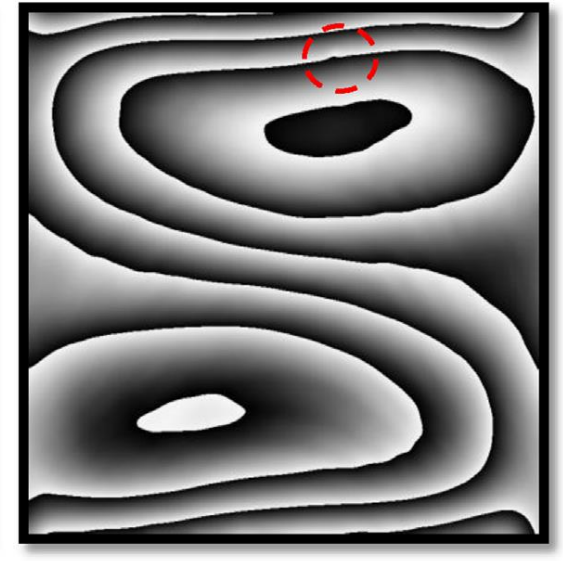

(c) phase by WFF at $\mathrm{t}=12.05 \mathrm{~h}$

Figure 2-5: Input is a noisy fringe; output is a filtered fringe pattern, phase map using FFT and WFF. Phase map using FFT and WFF

Studying the variation of the concentration in the entire domain is the main key to understand the double diffusive interaction phenomenon, which was one of the fundamental motivations of the SODI facilities. Figure 2-9 illustrates the concentration contours inside the primary cell during Run39 at the end of the thermodiffusion phase $(\mathrm{t}=12 \mathrm{~h})$ obtained by both the FFT and WFF methods. Despite the better result of WFF, it might be argued that the concentration map calculated by FFT provides a good enough agreement that reduces the calculation cost remarkably.

It must be noted that the most important parameter to be extracted from the thermodiffusion experiment is the maximum separation of the components, and according to this parameter, the Soret coefficient can be measured. In short, while having a clear view is helpful to analyze the 
pure diffusion in the system; it is essential to have the most accurate measurement of the concentration near both hot and cold walls in order to precisely measure $\mathrm{S}_{\mathrm{T}}$.

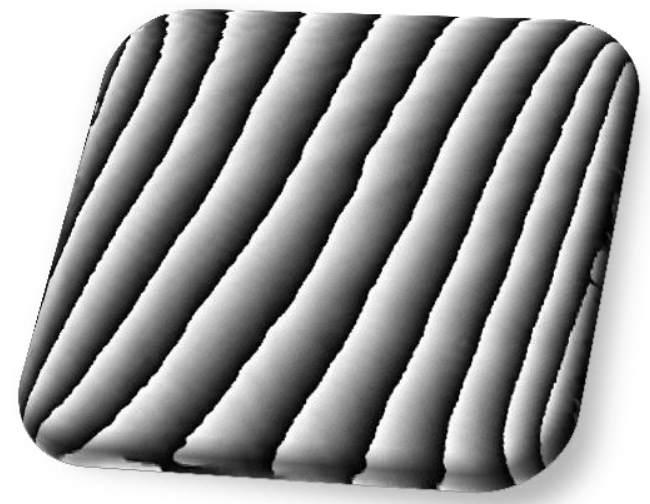

(a) a wrapped phase by FFT

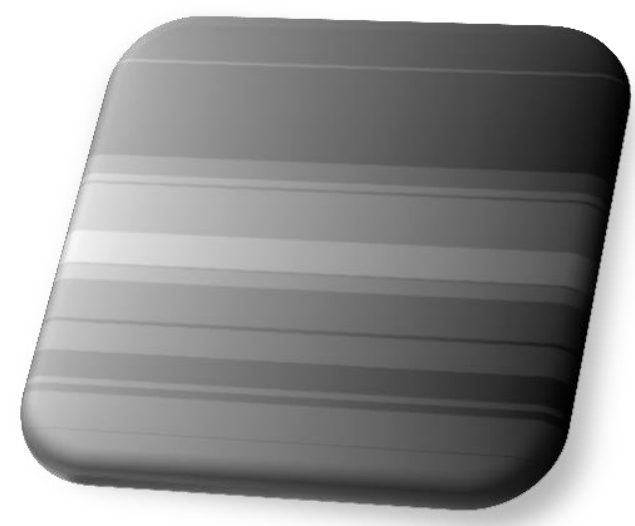

(c) unwrapped phase of part (a)

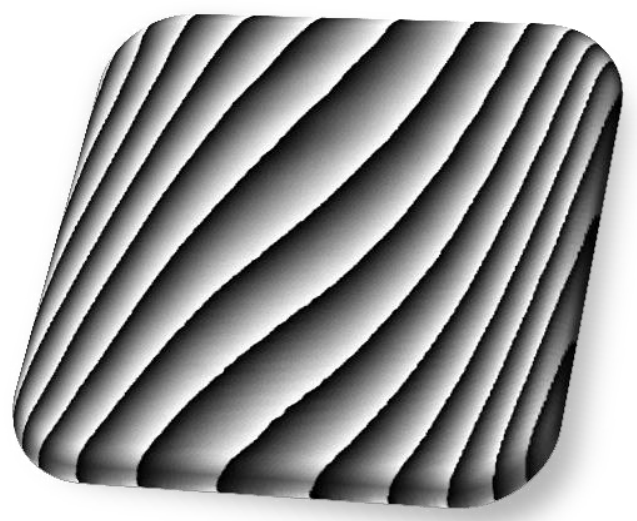

(b) wrapped phase by WFF

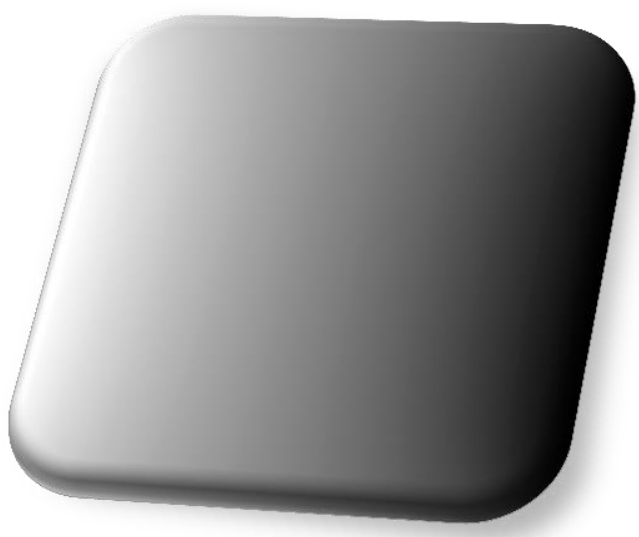

(d) unwrapped phase of part (b)

Figure 2-6: General results of the unwrap a phase map a) with and b)without badpixels or bad region close to boundaries showed in part $\mathrm{c}$ and $\mathrm{d}$ respectively

The last two parts of this figure demonstrate the same field one hour after the termination of the applied temperature difference, as the species were diffusing to establish a homogeneous mixture. The FFT results illustrate a wiggly pattern with stronger noise close to the walls, while the WFF results show isolines that are clear and smooth even at the corners of the cell. That the isolines can be distinguished trivially by WFF is an important result, since it is usually at the boundaries that the noise most affects the results. The maximum separation must be calculated 
using the extracted data inside the noisier region (close to walls). Thus, Figure 2-10a shows concentration variations along the thermal gradient direction close to the lateral wall of the cavity (line $\mathrm{L}_{2}$ in Figure 2-2), and Figure 2-10b illustrates the same but near the hot wall (line $\mathrm{L}_{4}$ in Figure 2-2).

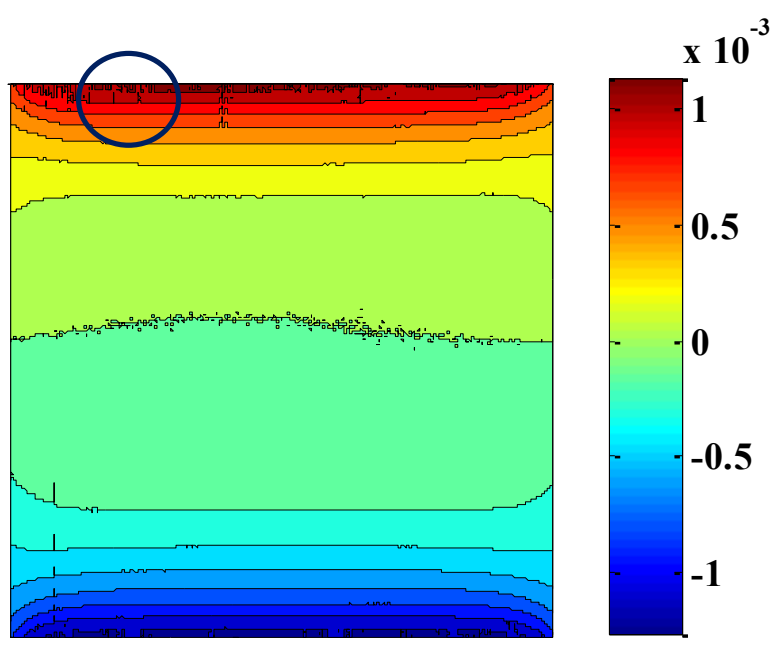

(a) Refractive index by FFT

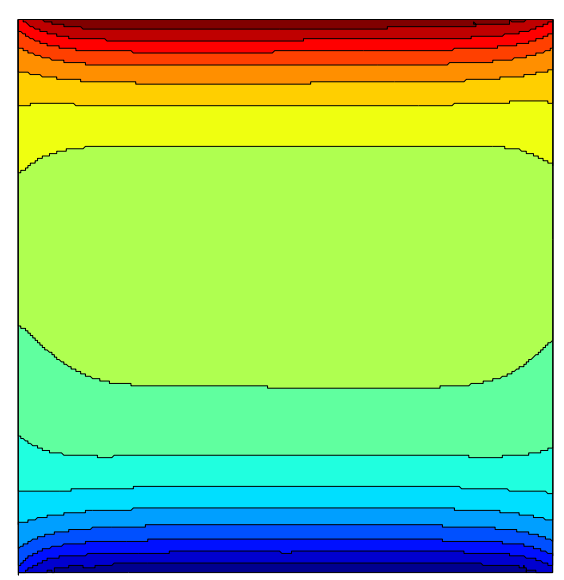

(b) Refractive index by WFT

Figure 2-7: Refractive index contour from a carrier fringes $(t=125 \mathrm{~s})$, wiggly domain in evident in the first part of the figure

The main oscillatory pattern is due to the external forced vibration imposed during Run33, which is traced by both methods. However, Figure 2-10a shows continuous fluctuations extracted using the FFT method, which do not exist in the real behavior that is extracted by WFF. It seems that the noise of the FFT method have a particular frequency and amplitude in this plot that is completely removed by WFF.
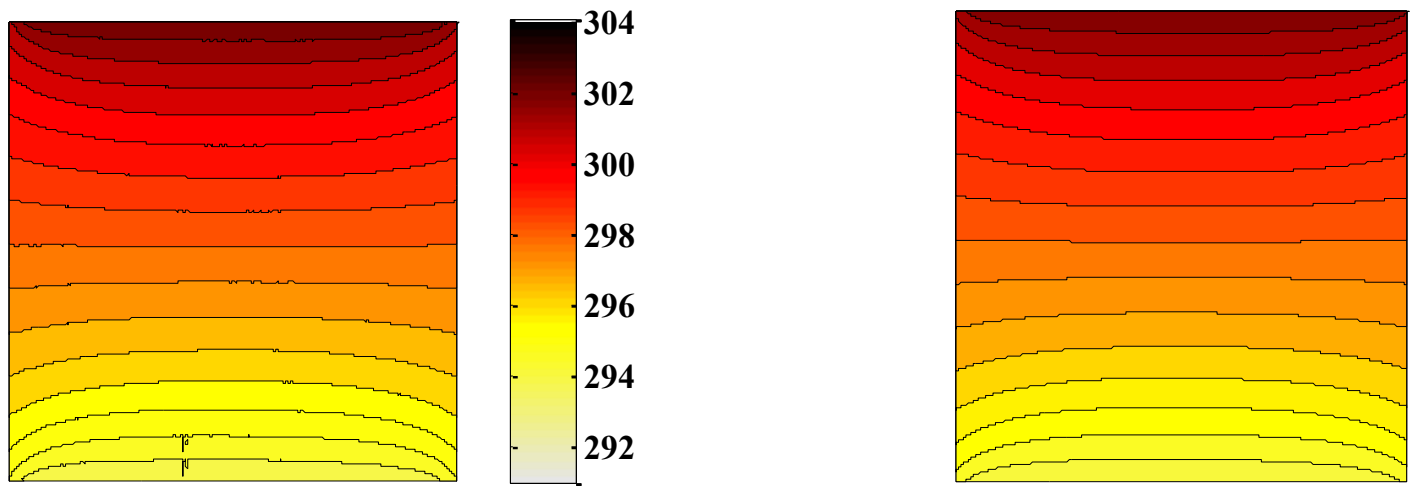

a) FFT
b)WFF

Figure 2-8: Temperature contours by different Fourier technique after two thermal times for binary cell Run2R

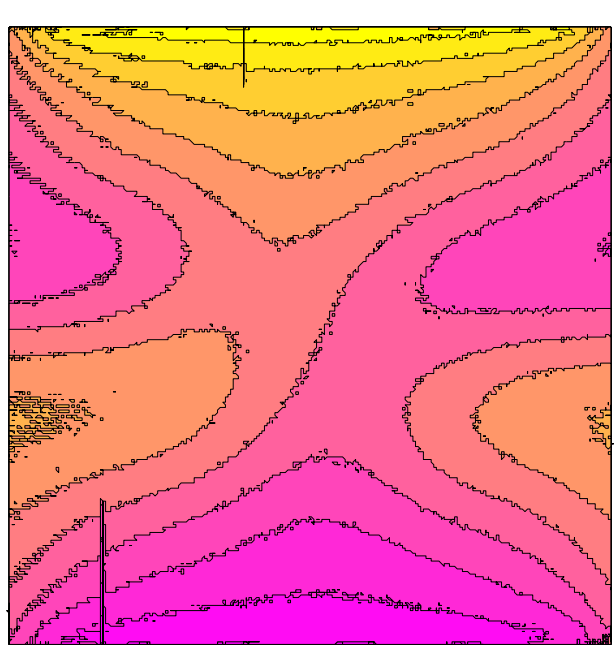

(a) FFT at $\mathrm{t}=12 \mathrm{~h}$

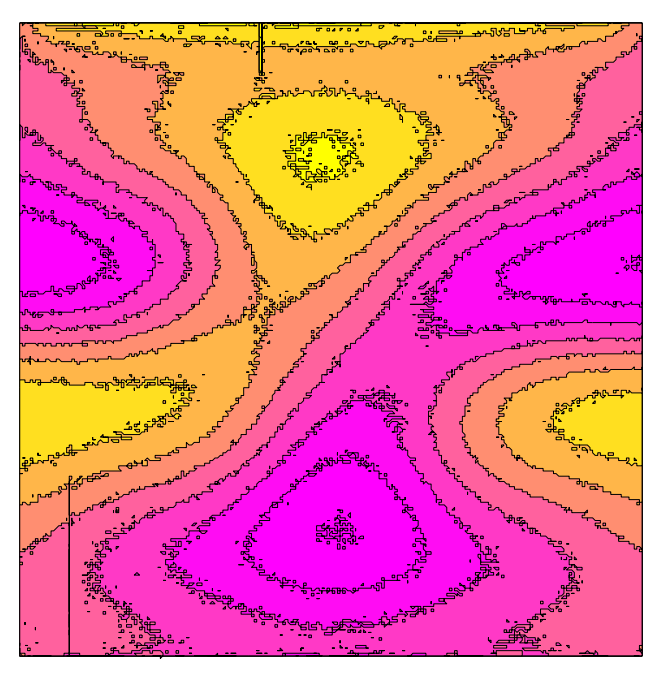

(c) FFT at t $=13 \mathrm{~h}$
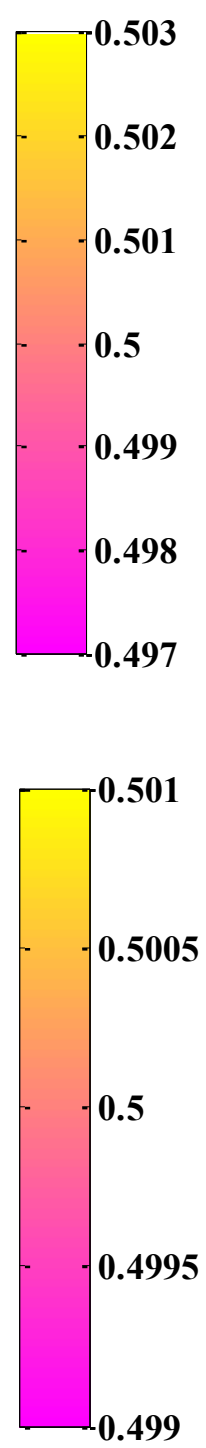

.501

0.5005

4995

0.499

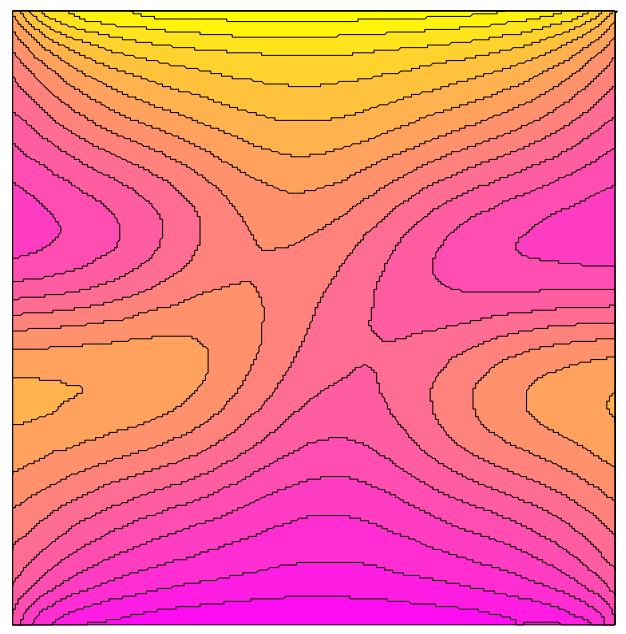

(b) WFF at $\mathrm{t}=12 \mathrm{~h}$

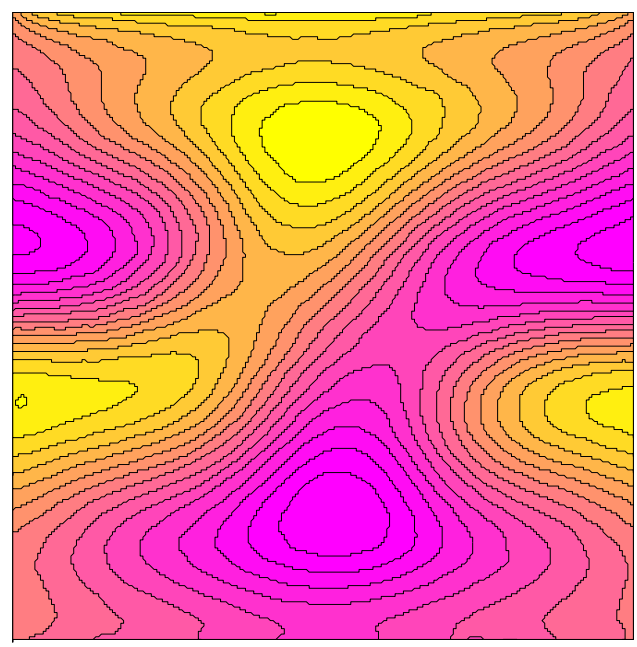

(d) WFF at t $=13 \mathrm{~h}$

Figure 2-9: IPA concentration contours in the cell for IVIDIL-Run33

The concentration near the hot and cold walls are the most important variable, which results in the quality of the FFT method being lower near these walls, as shown in Figure 2-10b. The closest row of the pixels to the hot wall $\left(\mathrm{FFT}_{1}\right)$ shows dominant fluctuations. This failure in the FFT method may be improved by analyzing the pixels at a larger distance from the wall. The mass fractions on the tenth pixel row (out of more than 800 row pixels) is also plotted in 
Figure 2-10 in green (captioned as $\mathrm{FFT}_{2}$ ), which displays a smoother and better performance at the middle region of the line $\mathrm{L}_{4}$. We observed that WFF provides a noticeable improvement of the concentration curve along the length of line $\mathrm{L}_{4}$. Although the concentration profile of the binary system extracted from the FFT method illustrated the wiggly domain, the profile pattern can still imitate the real one. However, for the case of the ternary mixtures the measurement conditions became more critical.

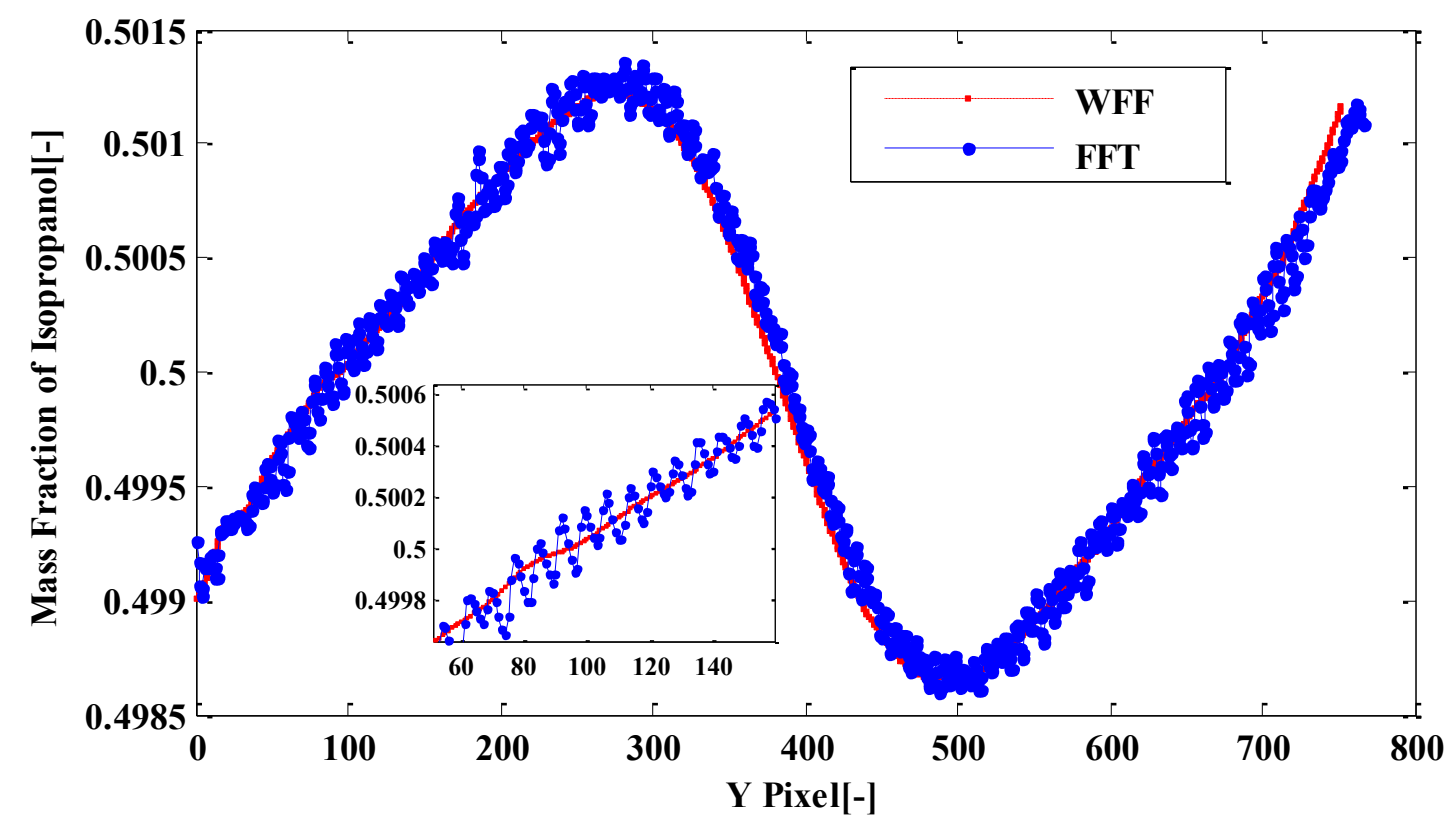

(a) along lines " $\mathrm{L}_{2}$ " :comparison between FFT and WFT 


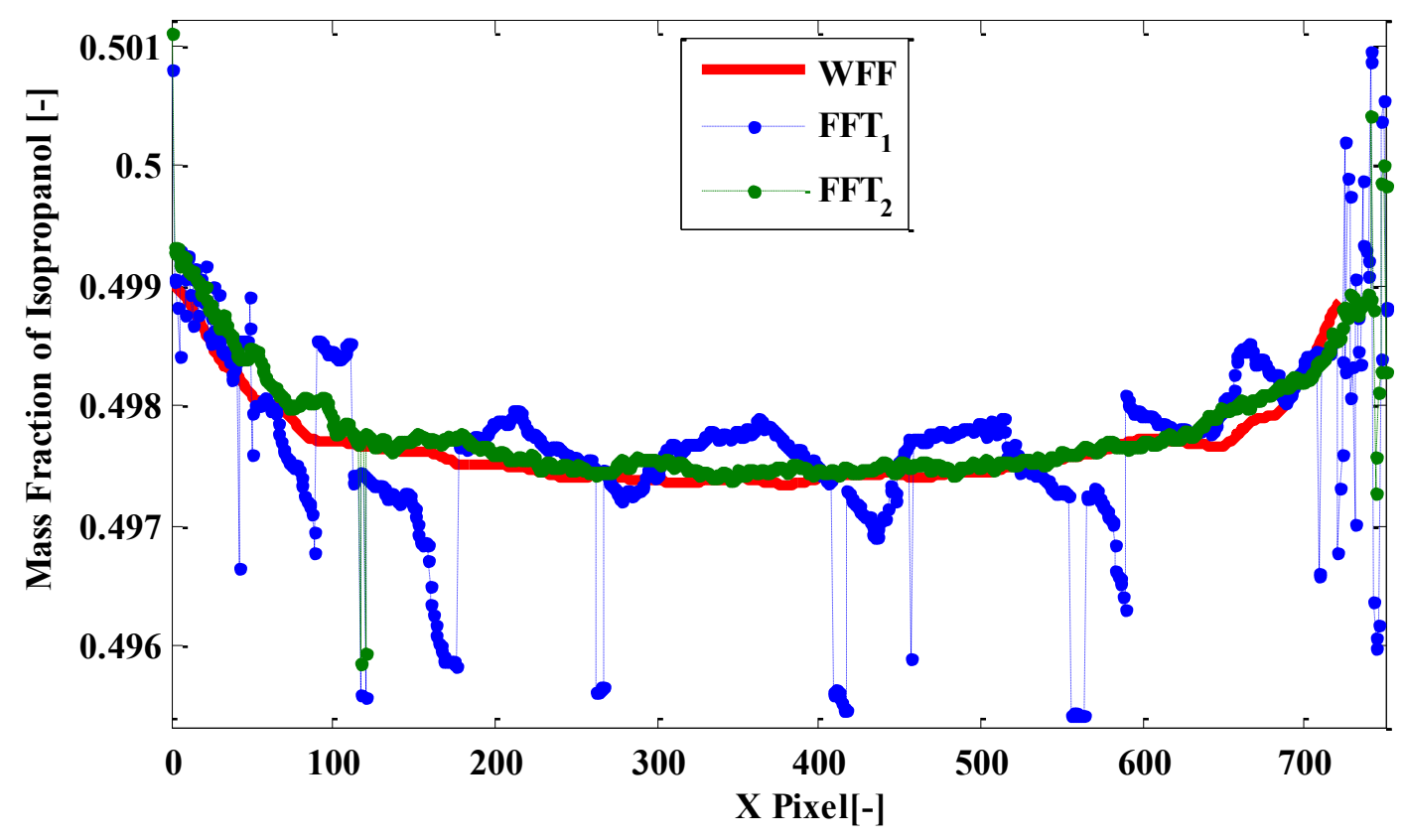

(b) along lines " $\mathrm{L}_{4}$ "

Figure 2-10: Results of mass fraction of IPA along different lines
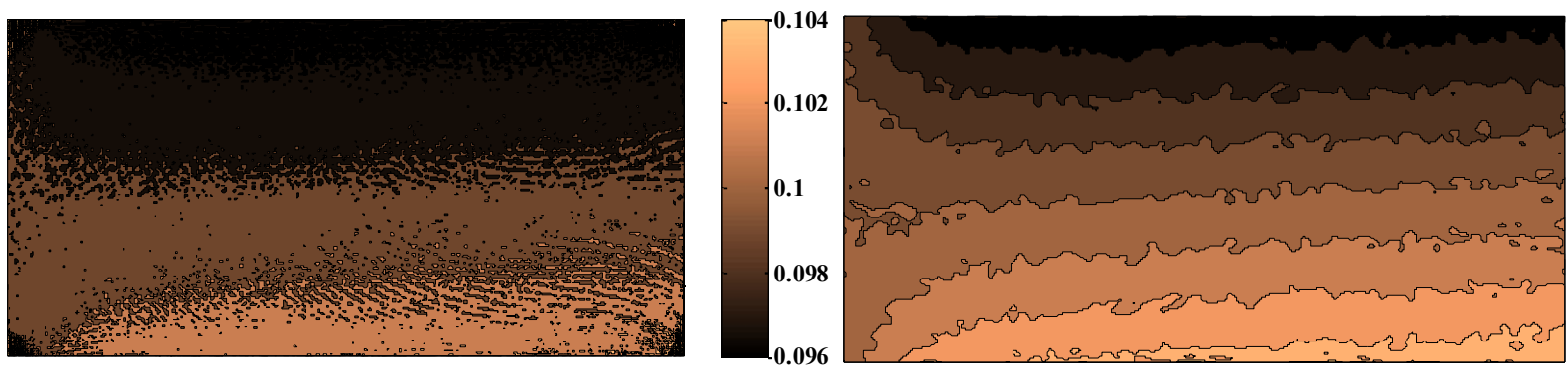

(a) THN by FFT

(b) THN by WFF
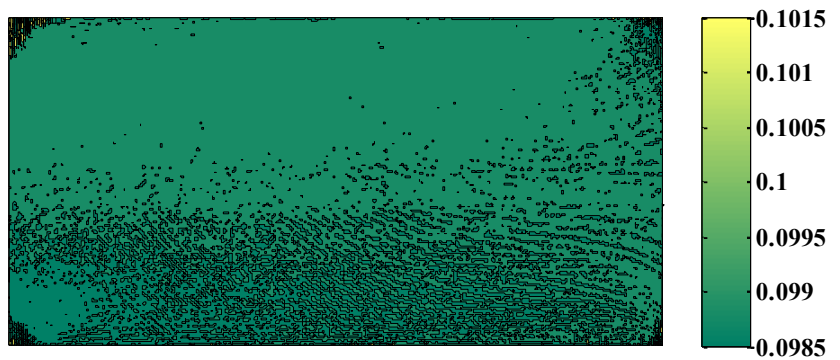

(c) IBB by FFT

(d) IBB by WFF

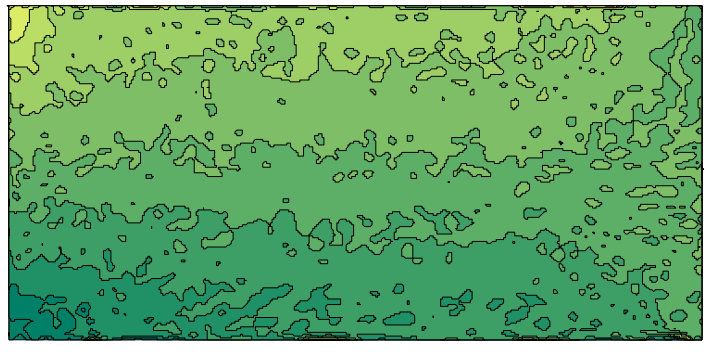


Figure 2-11: Concentration variation for DSC Run 12 at the end of thermodiffusion phase that shows the maximum separation in the system

The impact of the WFF on the concentration profiles proved more essential for ternary systems. Solving for the flow variables is a more complex process in case of a ternary mixture over a binary mixture, and the error is more pronounced as a result of the linked system of equations. The concentration distribution was completely different when comparing the FFT and WFF methods as demonstrated by Run12 from DSC shown in Figure 2-11. The variation of the species concentration was very small for this ternary mixture (less than 0.003 [wt]). The contours of the concentration obtained by the FFT method (Figure 2-11a,c) are not distinguishable from each other. Due to the lack of considerable separation of $\mathrm{C}_{12}$ in comparison with THN or IBB, these observations were even worse for the $\mathrm{C}_{12}$ contour. When WFF was employed (Figure 2-11b,d), the concentration gradient became discernable, but the errors were still not eliminated completely. However, the enhancement of WFF, especially for the case of THN, was substantial and was used to extract the needed parameters. The results show when the maximum separation in the domain is small $(<0.003$ [wt]), the FFT method cannot be employed as a reliable tool.

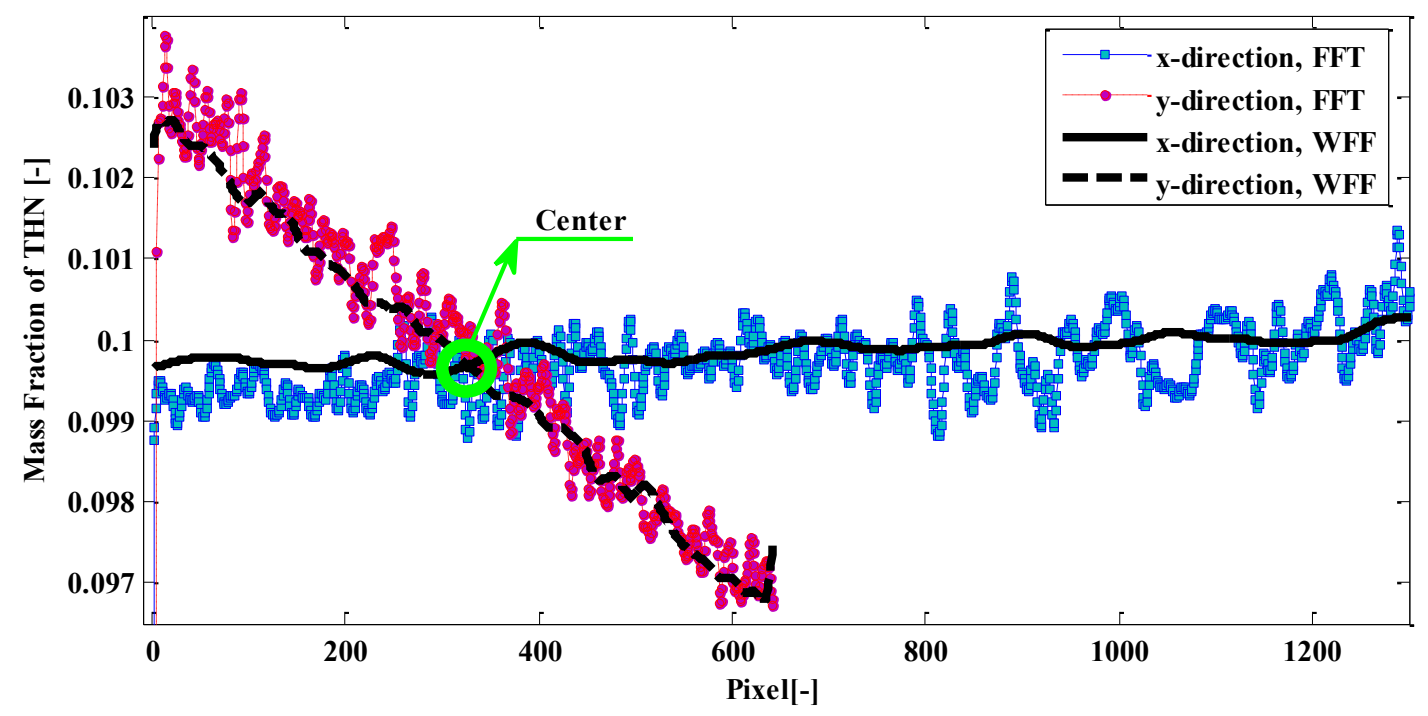

(a) along two perpendicular lines at the center of the cell and 


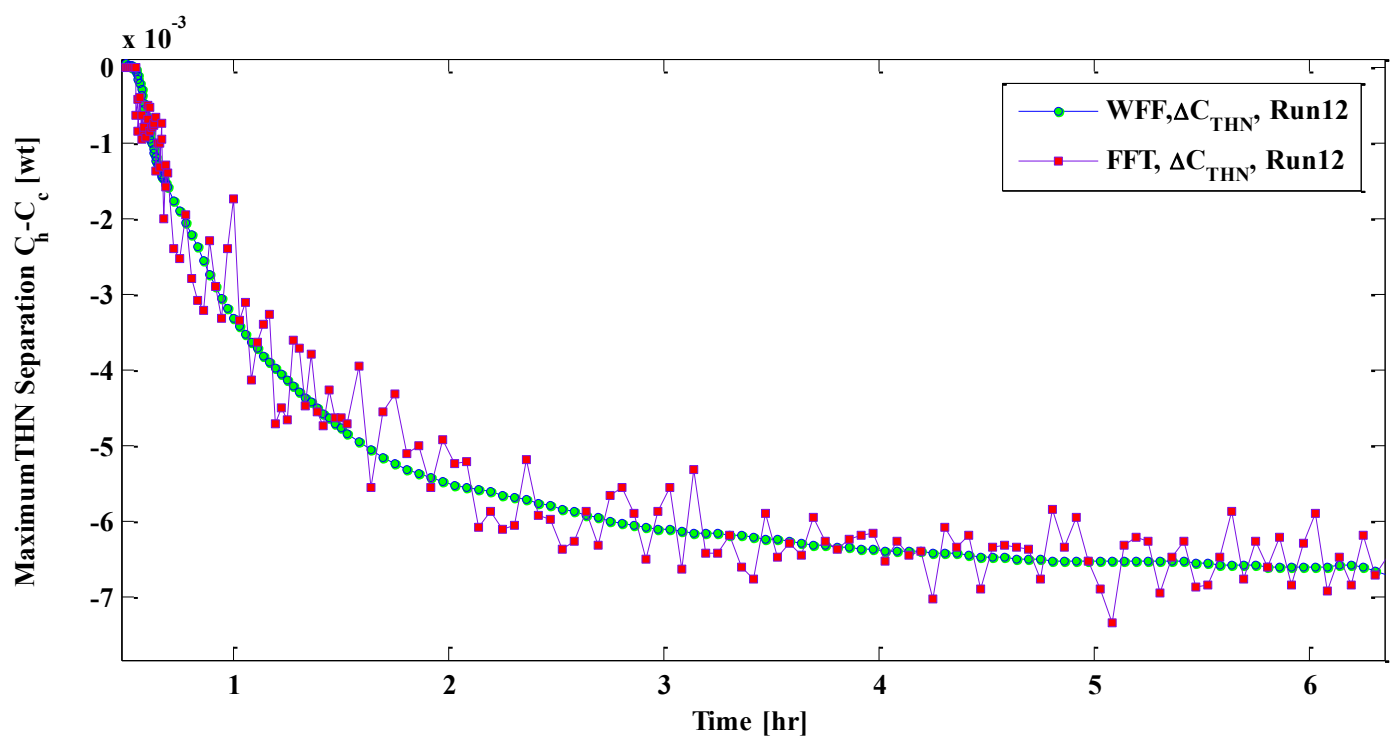

(b) maximum separation between hot and cold walls versus time

Figure 2-12: Comparison results of FFT and WFF for a ternary case

As mentioned earlier, the concentration profile at the center of the cavity is less affected by noise in the system. The concentration of THN along lines $L_{1}$ and $L_{3}$ are plotted in Figure 2-12a. The black solid and dashed lines correspond to the results of the WFF method and the FFT method, respectively. The fluctuation along the $\mathrm{x}$-direction is stronger than the $\mathrm{y}$-direction for both methods. The variation along the x-direction is plotted at the center of the cell, which must display the initial concentration of the components along $\mathrm{L}_{3}$ during the experiment. WFF extracts a more accurate result that satisfies the requirement of the initial concentration at the center of the cell $(\sim 0.1 \pm 0.0003[\mathrm{wt}])$.

The FFT result does not satisfy this condition as it deviates \pm 0.0012 from the required initial condition. In this case, the maximum separation in the system was less than 0.0065 [wt], which means that there was more than 18 percent variation in the intensity at any point in the domain using FFT. It is worth to mention that this error for the $\mathrm{C}_{12}$ is more than 40 percent, because the maximum separation of this component is about 0.0014 [wt], which is in the same order as error bar of the processing. The similar deflection along the y-direction was also noticeable using FFT, where the maximum concentration fluctuation compared to linear variation between the hot and cold walls along line $\mathrm{L}_{3}$ was 0.0016 . 
The MZI method provided the advantage of live tracing of the concentration distribution in the cell, a feature which is not accessible while using other experimental methods. Accurate capture of this capability is important for many different techniques of Soret effect measurements. Figure 2-12b illustrates the maximum temporal separation of THN in the ternary system during the thermodiffusion phase. Despite the more accurate values of Soret coefficient extracted with WFF, it must be noted that the FFT plot may represent the real trend of the maximum separation with a specific of fluctuation from reference of 0.0015 [wt] for the mixture in the normal condition. However, this error for the ternary mixtures at the critical conditions, such as choosing the points closer to walls to get the maximum possible separation or utilizing two lasers with close wavelengths in the experiment that results in more sensitive measurements, is more important and produces a deviation of 0.003 [wt] based on Figure 2-12a and Figure 2-11. This deviation in the ternary case may cause more than 30 to 50 percent error in measurement of the Soret coefficient if FFT is used.

\subsection{Conclusions}

The application of the two-dimensional windowed Fourier transform and its use for filtering during image processing of thermodiffusion interferometry experiments were investigated for the first time. A MATLAB program was developed that is facilitates the use of both FFT and WFT methods. In order to determine the impact the FFT and WFF methods on MZI thermodiffusion experiments, six different runs that were performed on board the ISS were analyzed according to both FFT and WFT. The overall requirements and different aspects of both FFT and WFT methods were discussed. It is shown that if WFT parameters are properly chosen, it can significantly reduce the noise from the fringe pattern of a MZI thermodiffusion experiment. The FFT method gives a reliable measurement of temperature for such a problem when a large

thermal gradient $(\sim 5 \mathrm{~K})$ exists between the cold and hot walls and when it is not essential to record precisely the temperature variations at different points in the domain. The comparison of the results shows that the WFT provides a noticeable improvement on the measurement of concentration. This improvement is more pronounced when dealing with very small variations in ternary systems. The FFT fails to extract a reasonable concentration profile for the ternary system. It is concluded that when the maximum separation in the domain is less than 0.003 [wt], 
the results of the FFT method is not reliable and may introduce more than 40 percent error in the measurements. This work provides the necessity of using the windowed Fourier transform method to study optical digital interferometry experiments involving both heat and mass transfer. The vital role of WFT method is indispensable in studying thermodiffusion in ternary systems. 


\section{Chapter 3: Theoretical and Experimental Measurement of Thermal Time for MZI to Study Heat and Mass Transfer Phenomena}

\subsection{Introduction}

When there is a constant temperature difference, various mixtures have different tendencies to separate. This tendency can be characterized by the thermodiffusion coefficients $\left(\mathrm{D}_{\mathrm{T}}\right)$, the Soret coefficient $\left(S_{T}\right)$, and the diffusion coefficient (D). Two of these coefficients must be measured experimentally, and the third one can be calculated as follows: $D_{T}=D \times S_{T}$ [94]. Accurate measurement of the thermodiffusion coefficient is important in oil exploration and in optimal oil recovery, since thermodiffusion causes diffusion fluxes in hydrocarbon reservoirs [64,110]. While the Soret coefficient is recognized as difficult to determine in ground conditions because of technical constraints in the control of liquid convection and convective instabilities [63], if the cell is heated from the top and the Rayleigh number is less than a critical value, then a pure thermodiffusion process occurs in the convectionless regime [41].

While most of the experimental methods can provide only the maximum separation in the field, the interferometry method can allow visualization of the front and side views of the experimental cell. These views result in plots of the temperature and concentration contours in the system versus time. The Mach-Zehnder interferometer is a setup employed to measure the relative phase shift between two collimated beams from a coherent light source. Mach-Zehnder interferometry (MZI) has been found to be an accurate and precise experimental model to monitor thermodiffusion inside a cubic cell when a thermal gradient is applied across two parallel sides of the cell. Processing the results of this experiment requires determining the stage of the experiment that temperature variations mainly govern the change of the refractive index of the mixture, known as thermal time, and the phase that the refractive index changes are due to the separation of the components, which is called diffusion, or thermodiffusion phase. We can separate these two phases according to the theoretical thermal time of the liquid mixture. However, as separation of the components starts when the thermal gradient is applied, during the 
thermal time the change of the refractive index in the system occurs because of the coupling of the heat and mass transfer in the system. Therefore, an accurate duration for this stage must be determined, otherwise the high ratio of separation at the beginning of experiment would be missed [112].

The thermal time must be calculated individually for different setups and experiments; nevertheless, the thermal time of the mixture can be calculated based on the physical properties of the materials and characteristics of the experiment according to the specific application of heat transfer. For instance, in order to study the effects of temperature on dormancy loss and germination [115] and for a practical engineering work [116], new time constants have been defined and used. In addition to what mentioned before new experimental models were developed to measure the thermal relaxation time constant $[117,118]$ for cases in which there were some limitations and constraints that had to be considered.

\subsection{Chapter Aims}

In the present chapter, a theoretical approach and experimental models are employed to estimate the thermal relaxation time of the optical digital interferometry experiment that deals with coupling between heat and mass transfer phenomena. Mach-Zehnder Interferometry was found to be accurate and precise experimental model to visualize the thermodiffusion phenomenon inside the cubic cell, when a thermal gradient is applied at two sides of a cell. Processing the results of this experiment requires distinguishing the phase of the experiment in which the temperature variation governs the change of the refractive index from the phase that the refractive index changes due to the separation of the components. We can separate these two phases according to the thermal time of the liquid mixture. In previous studies $\mathrm{L}^{2} / \chi(\mathrm{L}$ is the distance between hot and cold sides and $\chi$ is thermal diffusivity of the mixture) was used as the thermal time of the liquid. Due to a high separation rate at the beginning of the thermodiffusion process, a precise measurement of the thermal time can significantly affect the final result of the interferometry measurements. Here, theoretical and experimental evidence were used to estimate the proper thermal time for this experiment. We also discuss the influence on the experimental results of using these models. 


\subsection{Experimental Setup}

A cubic cell of $1 \mathrm{~cm}$ length in the $\mathrm{x}, \mathrm{y}$, and $\mathrm{z}$ directions was used. The geometry of a generalized Soret cell used on board the international space station (ISS) for the SODI (Selectable Optical Diagnostics Instrument) experiments is shown in Figure 3-1.

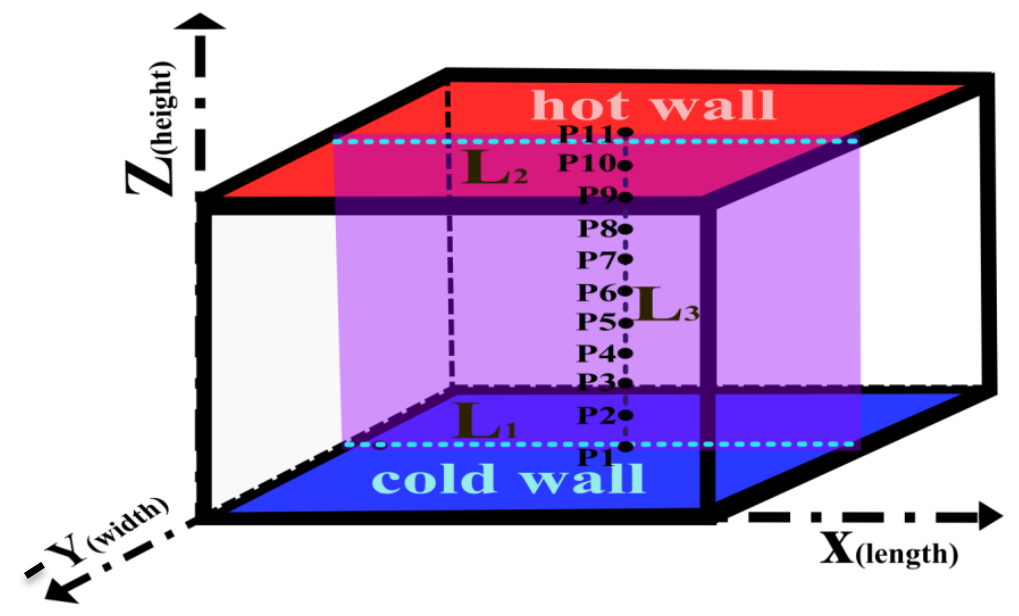

Figure 3-1: Scheme of the general Soret cell

Figure 3-2 shows the sketch of the optical apparatus that was employed as the MZI setup on board the ISS $[27,110,119]$. In this series of experiments, two laser beams of constant frequency and wavelengths of $\lambda=670 \mathrm{~nm}$ and $\lambda=935 \mathrm{~nm}$ were implemented. However, it must be noted that the results discussed herein were those from the laser with the wavelength of $\lambda=670 \mathrm{~nm}$.

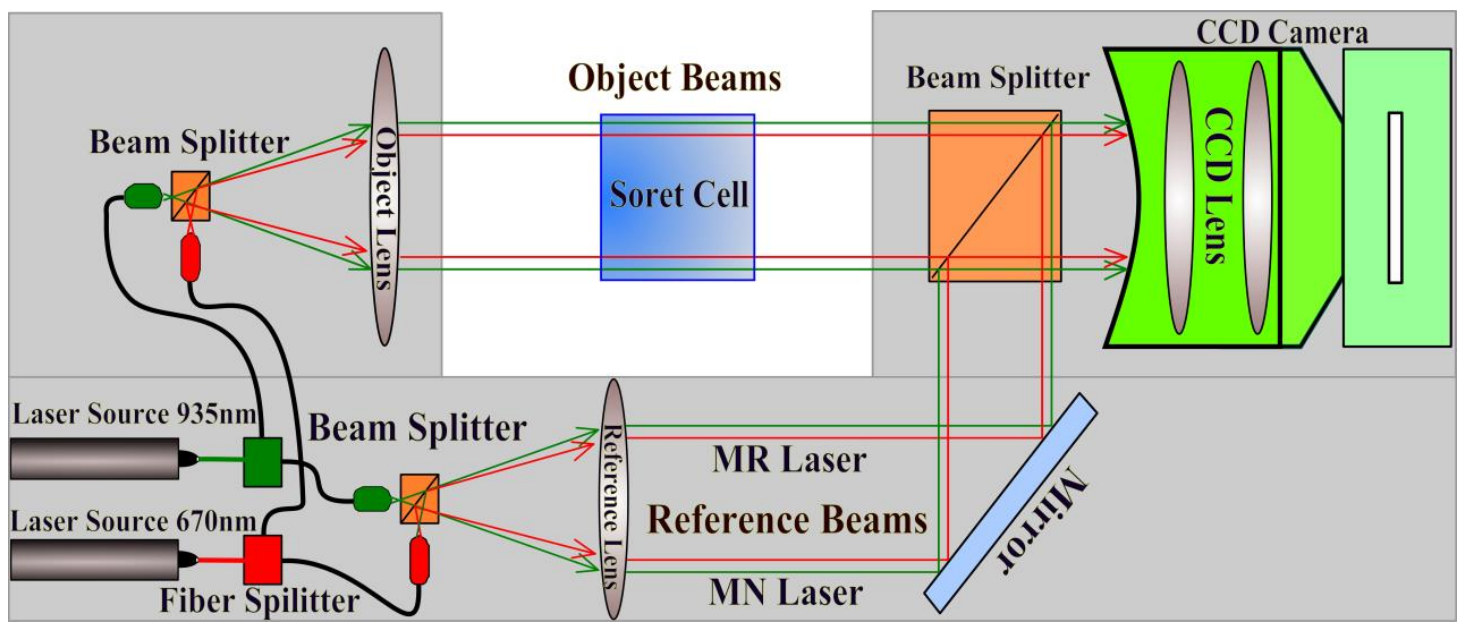

Figure 3-2: Optical digital interferometry scheme utilized with laser means of two different wavelengths as $\lambda=670 \mathrm{~nm}$ and $\lambda=935 \mathrm{~nm}$ on-board ISS 


\subsection{Experimental Sequences}

Each stage of the experiment lasts between 6 to 36 hours according to the diffusion time of the mixture. However, in some cases there was not enough time allotted for the experiment to reach the steady state condition. For example, a series of interferometry experiments to measure the Soret coefficient of binary and ternary mixtures was performed on board the international space station. In this experiment instead of the $27 \mathrm{~h}$ that would be required for the experiment, limitations on the ISS meant that only $12 \mathrm{~h}$ was devoted to the separation of the component for the thermodiffusion stage [112]. When a steady-state (maximum) separation is not obtained, a curve-fitting method should be implemented to estimate the diffusion coefficient. The next equation represents the analytical solution of the mass transfer phenomenon for a binary mixture at a zero-gravity condition. The curve fitting was performed based on the best estimates of values of $\mathrm{D}$ and $\mathrm{S}_{\mathrm{T}}$ in this equation that provide the closest matches with the experimental separation of the components [120].

$C(z, t)-C(z, 0)=-S_{T} C_{0}\left(1-C_{0}\right) \Delta T\left[\frac{1}{2}-\frac{z}{L}-\frac{4}{\pi^{2}} \sum_{n=2 k-1}^{\infty} \frac{1}{n^{2}} \exp \left(-n^{2} \frac{t}{\tau_{D}}\right) \cos \left(\frac{n \pi z}{L}\right)\right]$

In the above equation, $S_{T}$ and $D$ are unknown coefficients and $\tau_{D}$ is the diffusion time of the mixture $\tau_{\mathrm{D}}=\frac{\mathrm{L}^{2}}{\pi^{2} \mathrm{D}}$. It is worth noting that when steady separation is obtained, Equation 3-2 can be simplified as,

$S_{T}=-\frac{\Delta C_{s t}}{C_{0}\left(1-C_{0}\right) \Delta T}$

Accordingly, the first step of the experiment was to initialize mixture characteristics by reaching a uniform concentration and temperature inside the cavity. After that, the cell and interferometer were stabilized at the desired mean temperature. The next step was achieved by applying a thermal gradient across the cell. The thermal time which represents the time that thermal equilibrium is established is a function of cell's dimensions and the mixture thermal diffusivity $\tau_{\mathrm{th}}=\mathrm{L}^{2} / \chi$. Even though the separation of the components starts as soon as the thermal gradient is applied across the cell, it was assumed [18,27,110,114] that during the thermal time any change in the refractive index was due to the temperature variations in the system. This assumption was 
justified by comparing thermal and diffusion characteristic times. As the diffusion time in most of the cases is at least 100 times greater than the thermal time, neglecting the first few minutes of the diffusion time (less than 1\%) will not affect the maximum separation at the end diffusion time significantly. On the other hand, a high ratio of the total separation occurs at the beginning of the thermodiffusion phase. Thus, the thermal times for MZI thermodiffusion experiments must be either calculated or measured individually in order to obtain an accurate result.

\subsection{Methodology}

The main techniques used to process images was Fourier transforms followed by specific filtration and an unwrapping procedure [119], which resulted in a continuous phase map of each image. Then, this output was converted to show the refractive index variation $\mathrm{n}$ in the cell according to the following formula:

$\Delta n(x, y)=n(x, y)-n\left(x_{0}, y_{0}\right)=\frac{\lambda}{2 \pi L} \Delta \phi(x, y)$

where $\mathrm{L}$ is the optical path length, defined as the distance that the laser passes through the liquid in the cell. As shown in the above equation, the variation of refractive index is equivalent to the change in the optical phase. Since concentration and temperature contribute to the variation of refractive index for any given wavelength, the following relationship can be used to calculate $\Delta T$ and $\Delta \mathrm{C}$ separately.

$\Delta n_{i}(x, y)=\left(\frac{\partial n_{i}}{\partial T}\right)_{T_{0}, \lambda_{i}} \Delta T(x, y)+\sum_{j=1}^{w-1}\left(\left(\frac{\partial n_{i}}{\partial c_{j}}\right)_{T_{0}, \lambda_{i}} \Delta C_{j}(x, y)\right)$

where $\mathrm{w}$ is the number of components in the multicomponent mixture, and $\mathrm{j}$ and $\mathrm{i}$ are the indices that represent different components and various optical parameters for the $670 \mathrm{~nm}$ and $935 \mathrm{~nm}$ laser beams, respectively.

The temperature and concentration of the components were then calculated for a binary mixture from the refractive index variation during the thermal time and diffusion time according to Equations 3-5, and 3-6 respectively. 


$$
\begin{aligned}
& \Delta T(x, y)=\frac{\Delta n(x, y)}{\left(\frac{\partial n}{\partial T}\right)_{C_{0}}} \\
& \Delta C(x, y)=\frac{\Delta n(x, y)}{\left(\frac{\partial n}{\partial C}\right)_{T_{0}}}
\end{aligned}
$$

\subsubsection{Solution of Energy Equation for the MZI Experiment}

The following assumptions can be made for MZI thermodiffusion experiments under normal conditions:

- Convectionless regime

- Zero gravity

- Constant thermal conductivity

- Constant thermal diffusivity

- Zero heat flux at the non-heated walls;

In these conditions, the heat transfer between the hot and cold walls of the cell can be described by the 1-D transient heat conduction equation:

$$
\frac{\partial^{2} T}{\partial x^{2}}=\frac{1}{\chi} \frac{\partial T}{\partial t}
$$

If the reference of coordinate system is located at the cold wall, it is possible to express the initial and boundary conditions of the problem as a function of the temperature as follows:

$$
\begin{array}{ll}
T_{(x, 0)}=T_{\text {mean }} & \\
T_{(0, t)}=T_{\text {cold }} & t>0 \\
T_{(L, t)}=T_{\text {hot }} & t>0
\end{array}
$$

Considering the non-dimensional temperature and length as:

$$
\theta=\frac{T-T_{\text {mean }}}{T_{\text {hot }}-T_{\text {mean }}}, X=\frac{z}{L}
$$

Using the mentioned non-dimensional terms in Equation 3-7, the non-dimensional form of heat transfer can be shown as, 


$$
\frac{d^{2} \theta}{d X^{2}}=\frac{d \theta}{d \tau_{t h}}
$$

where $\tau_{\mathrm{th}}$ is non-dimensional time as $\tau_{\mathrm{th}}=\frac{\chi \mathrm{t}}{\mathrm{L}^{2}}$. At $\tau_{\mathrm{th}}=1$, the solution of the above partial differential equation (PDE) represents the thermal behavior of the system at steady state. Thus, the corresponding value $\tau_{\mathrm{th}}=1 \mathrm{can}$ be used for general heat transfer problems when there is no time constraints or limitations. However, as a result of mass flux during the thermal time of the thermodiffusion process, a precise calculation of the thermal time is required. Otherwise, a noticeable amount of separation at the beginning of the diffusion process will be neglected.

The above condition demonstrates a non-homogeneous PDE problem. Thus, the non-dimensional temperature can be expressed in the form of $\theta_{(X, \tau)}=\Psi_{(X, \tau)}+\phi_{(X)}$ to satisfy the non-homogeneous boundary conditions. The non-dimensional initial and boundary conditions are presented below:

$$
\begin{aligned}
& \theta_{(0, \tau)}=\Psi_{(0, \tau)}+\phi_{(0)}=-1 \Rightarrow \Psi_{(0, \tau)}=0, \phi_{(0)}=-1 \\
& \theta_{(1, \tau)}=\Psi_{(1, \tau)}+\phi_{(1)}=1 \Rightarrow \Psi_{(1, \tau)}=0, \phi_{(1)}=1 \\
& \theta_{(X, 0)}=\Psi_{(X, 0)}+\phi_{(X)}=0 \Rightarrow \Psi_{(X, 0)}=-\phi_{(X)}
\end{aligned}
$$

Substituting $\theta_{(X, \tau)}=\Psi_{(X, \tau)}+\phi_{(X)}$ in the main equation would result two independent differential equations that have homogeneous conditions:

$$
\begin{aligned}
& \frac{\partial^{2} \Psi}{\partial X^{2}}+\frac{d^{2} \phi}{d X^{2}}=\frac{\partial \Psi}{\partial \tau} \Rightarrow \text { (1) } \frac{\partial^{2} \Psi}{\partial X^{2}}=\frac{\partial \Psi}{\partial \tau} \\
& \text { (2) } \frac{d^{2} \phi}{d X^{2}}=0 \Rightarrow \phi_{(X)}=2 X-1
\end{aligned}
$$

Equation 3-12 can be solved using the separation of variables $\left(\Psi_{(x, \tau)}=F_{(x)} \cdot G_{(\tau)}\right)$, where $F=A \cdot \sin (\alpha X)$ and $G=C \cdot \exp \left(-\alpha^{2} \tau\right)$. Thus, $\Psi_{(X, \tau)}=c t n_{p} \cdot \sin (\alpha X) \exp \left(-\alpha^{2} \tau\right)$, in which $\operatorname{ctn}_{\mathrm{p}}$ is a new unknown coefficient and $\lambda$ is $p \pi$ where $\mathrm{p}$ varies from 1 to infinity. In order to calculate $\mathrm{ctn}_{\mathrm{p}}$ the concept of orthogonal functions concepts should be employed for the following equation: 


$$
\begin{aligned}
& \Psi_{(X, 0)}=1-2 X \sum_{n=1}^{\infty} C_{n} \cdot \sin (n \pi X) \stackrel{\times \sin (\mathrm{m} \pi \mathrm{x})}{\longrightarrow} \int_{0}^{1}(1-2 X) \sin (m \pi X) d X= \\
& \int_{0}^{1} \sum_{n=1}^{\infty} C_{n} \cdot \sin (n \pi X) \sin (m \pi X) d X \stackrel{{ }_{n=m}}{\longrightarrow} \int_{0}^{1} \sum_{n=1}^{\infty} C_{n} \cdot \sin ^{2}(n \pi X) d X
\end{aligned}
$$

Consequently,

$$
C_{n}=\frac{\int_{0}^{1}(1-2 X) \sin (n \pi X) d X}{\int_{0}^{1} \sum_{n=1}^{\infty} \sin ^{2}(n \pi X) d X}=\frac{2\left(1+(-1)^{n}\right)}{n \pi}
$$

At the end, by using the dimensional parameters instead of non-dimensional numbers, the temperature variation versus length of the cell would be found as:

$$
T_{(x, t)}=2\left(T_{\text {hot }}-T_{\text {mean }}\right)\left[\frac{z}{L}-\frac{1}{2}+\sum_{n=1}^{\infty} \frac{2\left(1+(-1)^{n}\right)}{n \pi} \exp \left(\frac{-n^{2} \pi^{2} \chi t}{L^{2}}\right) \cdot \sin \left(\frac{n \pi z}{L}\right)\right]+T_{\text {mean }}
$$

At $\mathrm{t}=\infty$, Equation 3-15 can be simplified to a linear variation between the cold and hot walls as presented in Equation 3-16:

$$
T_{(x, \infty)}=2\left(T_{\text {hot }}-T_{\text {mean }}\right) \frac{z}{L}-T_{\text {hot }}+2 T_{\text {mean }} \frac{T_{\text {mean }}=\frac{T_{\text {hot }}+T_{\text {cold }}}{2}}{\longrightarrow} T_{(x, \infty)}=\frac{T_{\text {hot }}-T_{\text {cold }}}{L} z-T_{\text {cold }}
$$

In order to measure the Soret coefficient of any mixture, the maximum separation of the components due to the temperature difference must be obtained. The maximum separation occurs near the hot and cold walls of cavity as shown by lines $L_{1}$ and $L_{2}$ in Figure 3-1.

\subsubsection{Theoretical Model to Determine Thermal Time for Mixtures with Negative Soret Coefficients}

Two different situations may occur during the MZI thermodiffusion experiment for different compositions of a mixture: First, a negative Soret coefficient represents a stable condition (most frequently occurs for the mixtures), and second, a positive Soret coefficient that means an unstable condition of heat and mass transfer in the system. For the first case (negative Soret coefficient means the heavier component moves towards the hot side), both application of the temperature gradient and separation of the components increase the absolute value of the 
refractive index difference between the hot and cold sides. By considering the thermal time equal to $\mathrm{t}_{\mathrm{th}}=\mathrm{L}^{2} / \chi, \Delta \mathrm{n}$ measured during this period caused by temperature variation and separation, the entire change of refractive index that is measured after this time may not give an accurate $\Delta \mathrm{C}$. It is because a portion of the separation during the thermal time is not considered. This results in an overestimation of the temperature variation during the thermal time and an underestimation of the concentration variation during the diffusion time. On the other hand, if we consider the thermal time before the steady-state condition $\left(t_{\mathrm{th}}=\frac{\mathrm{L}^{2}}{\chi}\right)$, during the thermal time there would be a $\Delta \mathrm{n}$ that is not considered and will be counted during the diffusion time, a process that is not precise either. Thus, if we set the amount of refractive index change that is not considered equal to the amount that is added correctly, we end up with the accurate time that is need to be determined for the MZI thermodiffusion thermal time measurement. During the thermal time, there was a deviation from the linear temperature variation between walls (linear variation of $\Delta \mathrm{T}$ occurred at $t_{\mathrm{th}}=\frac{\mathrm{L}^{2}}{\chi}$ ). Figure 3-1a shows the temperature variation at eleven points along the $\mathrm{z}$ direction of the cell represented in Figure 3-1. It is evident that at the second half of the thermal time, there is almost no variation of temperature in any of the locations. However, due to the sensitivity of the refractive index to small temperature changes, even this small temperature variation should be studied in detail. Moreover, the non-linear temperature deviation illustrated in Figure 3-3b suggests that the steady-state condition occurs at different times at the various locations along the z-axis. In order to determine the critical locations, the non-dimensional time $\left(t_{\mathrm{th}}=\frac{\mathrm{L}^{2}}{\chi}\right)$ was chosen at which $99.99 \%$ of the steady non-dimensional temperature was achieved, as plotted in Figure 3-4 (which means the time that $\theta-\theta_{\mathrm{th}}=0.0001$ is a function of location).

Locations $X=(z / L)=1 / 4$ and 3/4 reached thermal steady condition later than the other locations in the field. Thus, we have calculated the thermal time for these critical points. The temperature difference between these two points is

$$
T_{(3 L / 4, t)}-T_{(L / 4, t)}=2\left(T_{\text {mean }}-T_{(L / 4, t)}\right)=\Delta T\left[\frac{1}{2}-\frac{8}{\pi} \sum_{n=4 k-2}^{\infty} \frac{(-1)^{k+1}}{n} \exp \left(\frac{-n^{2} \pi^{2} \chi t}{L^{2}}\right)\right]
$$


where $\mathrm{k}$ is $1,2,3, \ldots$ The deviation of the temperature difference in Equation 3-17 (of locations $\mathrm{X}=(\mathrm{z} / \mathrm{L})=1 / 4$ and $3 / 4)$ from its value at the thermally steady time can be expressed as in Equation 3-18:

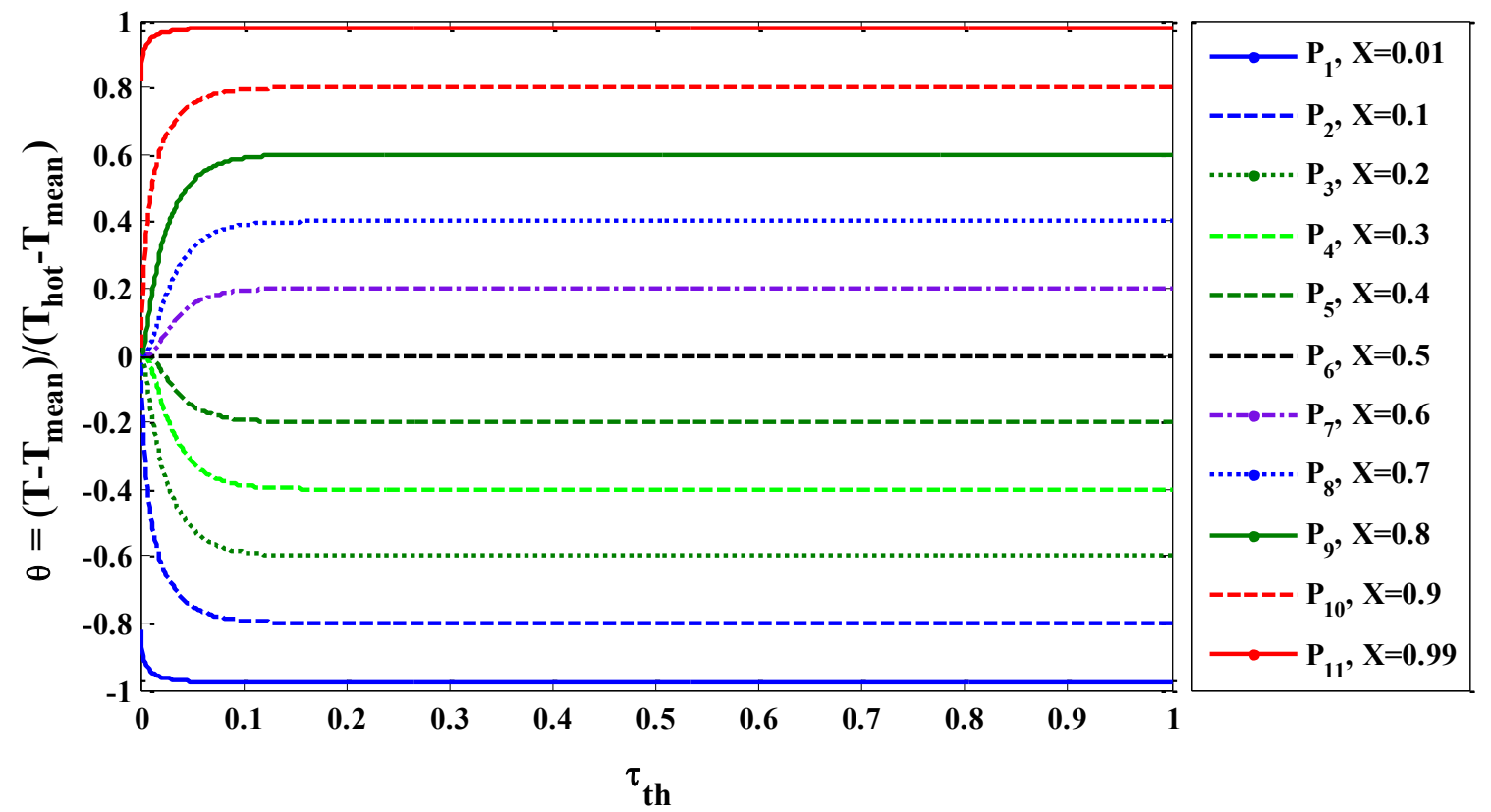

(a) Temperature variation at different locations along z-direction

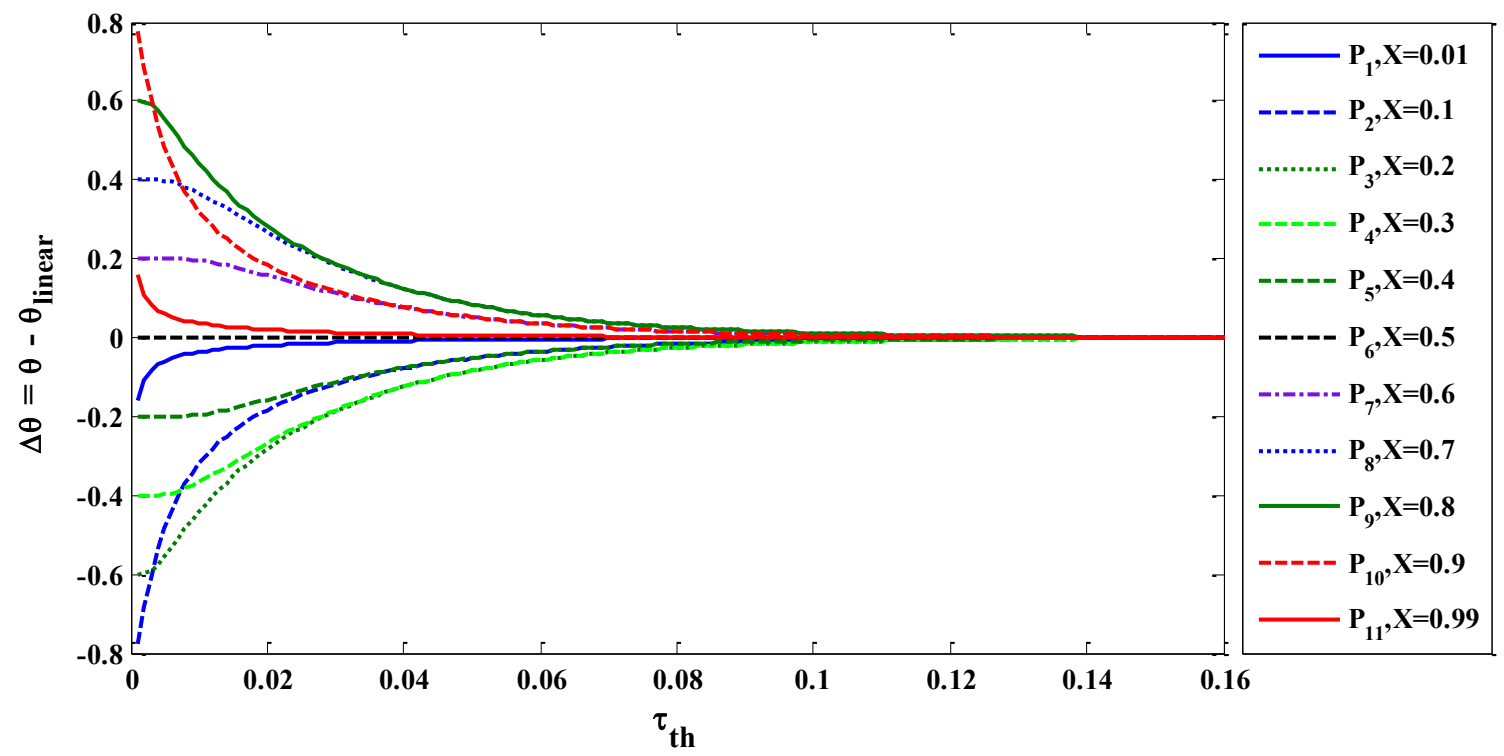

(b) Temperature deviation from the linear variation at the steady condition at different locations along z-direction

Figure 3-3: Non dimesional Temperature variation at various locations 


$$
\left(T_{(3 L / 4, \infty)}-T_{(L / 4, \infty)}\right)-\left(T_{(3 L / 4, t)}-T_{(L / 4, t)}\right)=\frac{8\left(T_{h o t}-T_{\text {cold }}\right)}{\pi} \sum_{n=4 k-2}^{\infty} \frac{(-1)^{k+1}}{n} \exp \left(\frac{-n^{2} \pi^{2} \chi t}{L^{2}}\right)
$$

On the other hand, it is an accurate assumption that separation of the components starts when $99.99 \%$ of the applied temperature gradient has been formed the hot and cold walls of the entire domain. According to equation 3-18, this situation happens at $\tau_{\mathrm{th}}=0.221$. In addition, Equation 3-1 can be rewritten as follows for the same location:

$C\left(L / 4, t_{c}\right)-C\left(3 L / 4, t_{c}\right)=-S_{T} C_{0}\left(1-C_{0}\right) \Delta T\left[\frac{1}{2}-\frac{8}{\pi^{2}} \sum_{n=2 k-1}^{\infty} \frac{1}{n^{2}} \exp \left(-\frac{n^{2} \pi^{2} D t}{L^{2}}\right) \cos \left(\frac{n \pi}{4}\right)\right]$

If the thermal time for the MZI thermodiffusion experiment is considered before $t_{\mathrm{th}}=\frac{\mathrm{L}^{2}}{\chi}$, there is a change in the refractive index due to the temperature during diffusion that belongs to the thermal time, and conversely there is also a change in the refractive index due to separation during the thermal time that belongs to the diffusion time. If we set these two changes equal to each other, we can find the exact thermal time for the MZI experiment.

$$
\begin{aligned}
& \left|\left(C_{\left(L / 4, t_{c}\right)}-C_{(L / 4,0)}\right) \frac{\partial n}{\partial C}\right|=\left|\left(T_{\left(L / 4, t_{T}\right)}-T_{(L / 4, \infty)}\right) \frac{\partial n}{\partial T}\right| \\
& \frac{\partial n}{\partial C} / \frac{\partial n}{\partial T}=\left(\Delta T \mid \begin{array}{l}
t_{T} \\
t_{T_{0}}=0
\end{array}\right) /\left(\left.\Delta C\right|_{\left.\right|_{c_{0}} ^{t_{c}}=0} ^{t_{t}}\right), t_{T}=t_{c}+0.221 \times \tau_{t h} \\
& \frac{\partial n / \partial C}{\partial n / \partial T}=\frac{\sum_{n=4 k-2}^{\infty} \frac{-8}{n \pi} \exp \left(\frac{-n^{2} \pi^{2} \chi t_{T}}{L^{2}}\right)}{S_{T} C_{0}\left(1-C_{0}\right)\left(\frac{1}{2}-\frac{8}{\pi^{2}} \sum_{n=2 k-1}^{\infty}\left(\frac{1}{n^{2}} \exp \left(-\frac{n^{2} \pi^{2} D\left(t_{T}-0.221 \times L^{2} / \chi\right)}{L^{2}}\right) \cos \left(\frac{n \pi}{4}\right)\right)\right)}
\end{aligned}
$$

In order to solve Equation 3-22, the graphical or iterative method can be used for any specific mixture with negative Soret coefficient. Interestingly, the solution is not a function of the temperature difference in the system, but it is a function of the thermal diffusivity, the diffusion coefficient, and the distance between the cold and hot sides. Figure 3-5 illustrates the graphical solution for Equation 3-22 in which the test mixture is water/isopropanol (10\% initial mass 
fraction of IPA) in a cubical cavity that measures $1 \mathrm{~cm}^{3}$. At $216 \mathrm{~s}$, the refractive index difference is the amount that must be considered for the first step of the experiment in order to obtain accurate results for the concentration contours. One may argue that the $S_{\mathrm{T}}$ used in this thermal time modeling is by itself a function of the thermal time, even though it is used as a parameter in Equation 3-22.

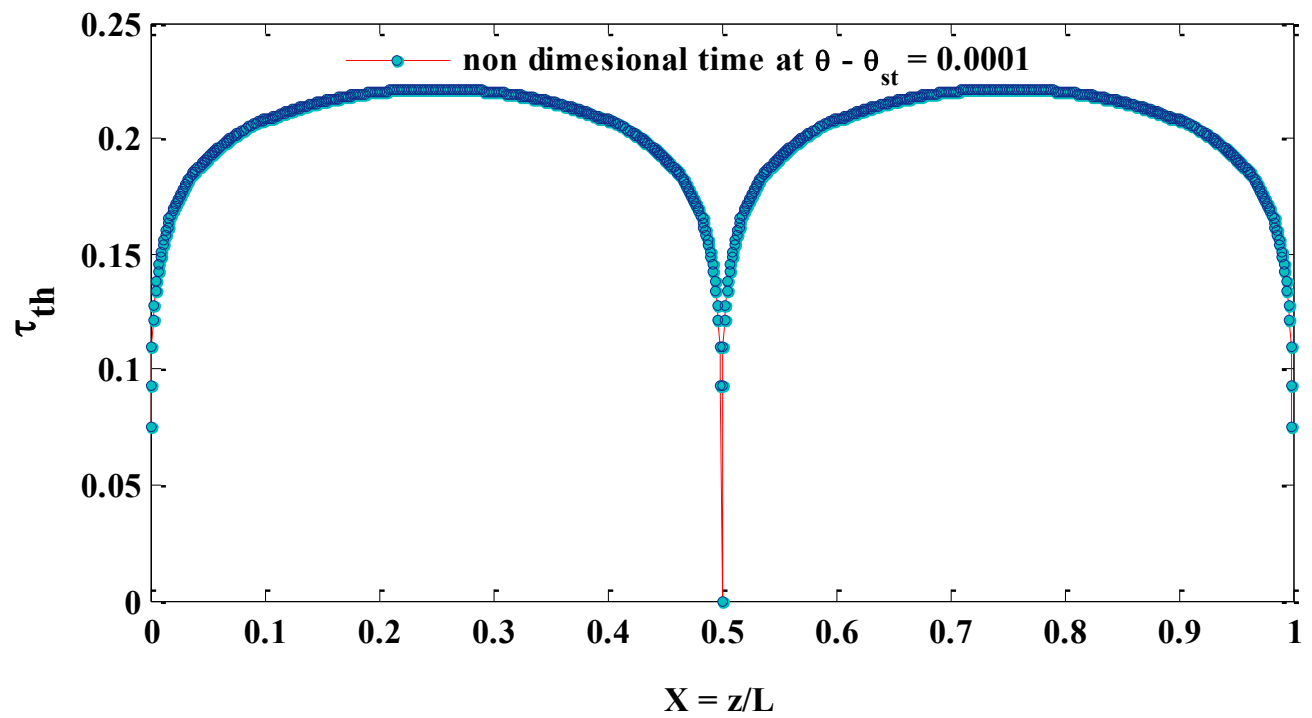

Figure 3-4: Temperature deviation from the linear variation at the steady condition at different location along z-direction

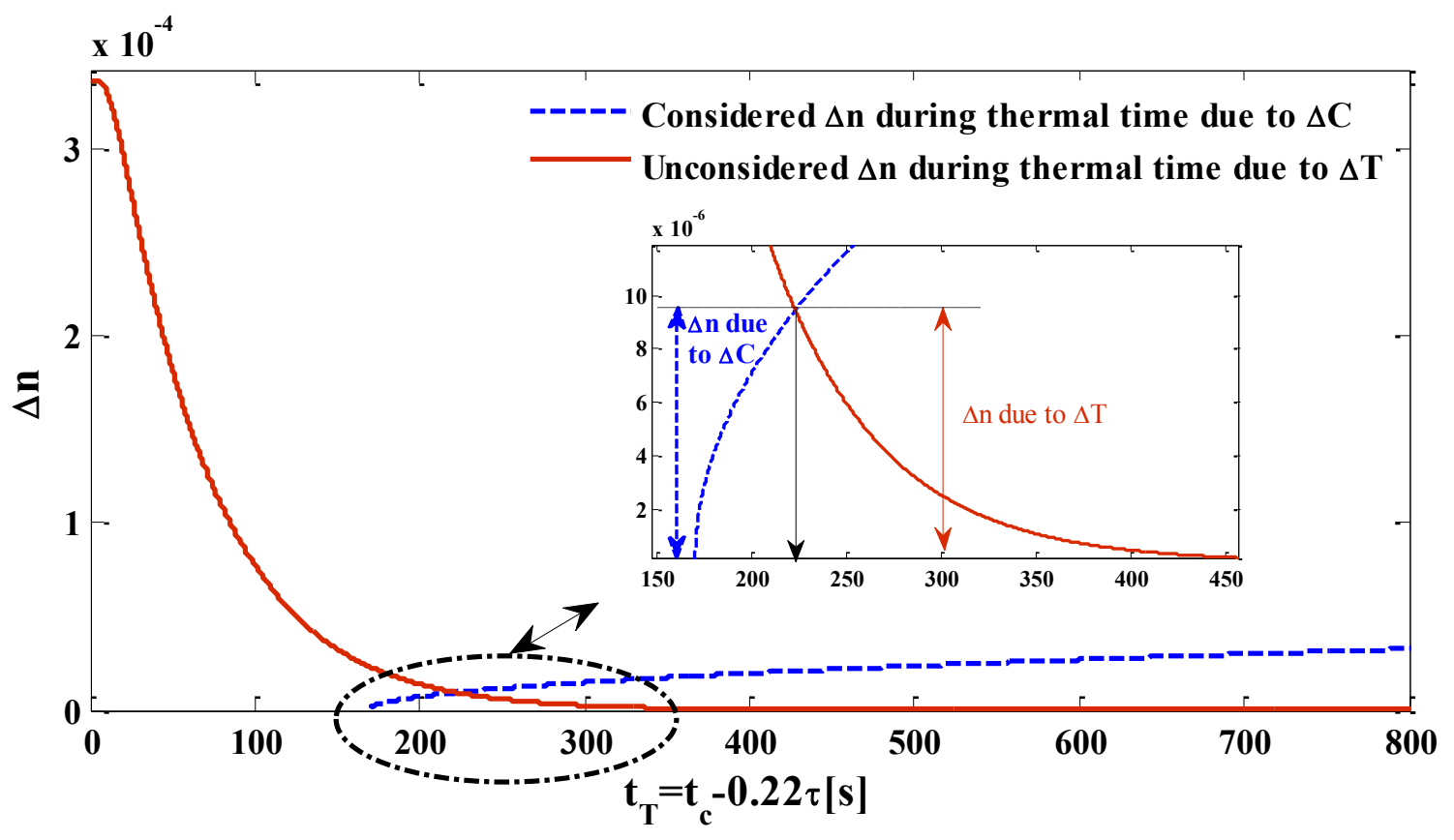

Figure 3-5: Refractive index versus time during $\mathrm{L}^{2} / \chi$ 
In this case, it must be noted that the Soret diffusion coefficient adjusts the slope of the diagram, which does not change the final solution of this problem noticeably. For instance, we found that for our sample mixture (water/IPA), if the $S_{\mathrm{T}}$ is assumed to be two times greater ( $\mathrm{S}_{\mathrm{T} \text {,greater }}$ $\left.=16.95 \times 10^{-3}\right)$ or smaller $\left(\mathrm{S}_{\mathrm{T} \text {,smaller }}=4.24 \times 10^{-3}\right)$ than its benchmark value $\left(8.47 \times 10^{-3}\right)$, the final calculated thermal time does not change more than $26 \mathrm{~s}$ from the one that calculated with an accurate $S_{\mathrm{T}}$. This means that there is less than a $5 \%$ relative variation in comparison with the value of $t_{t h}$. The proposed value of the thermal time that is obtained by this method was more than three times smaller than $t_{\text {th }}$. The same argument can be made for the effect of varying the initial time that the separation is started, $t_{c}$. If the separation starts when $90 \%$ (or 99.999\%) of the assigned temperature variation is built up across the cell, the final answer of Equation Equation 3-22 varies about $17 \mathrm{~s}$ (or $30 \mathrm{~s}$ ). The accuracy of this model is not a strong function of the initial estimates of unknown parameters. The model is, therefore, a solid and reliable method to calculate the required thermal time for the Soret diffusion coefficient measurement of a mixture with a negative Soret coefficient using optical interferometry.

\subsubsection{Experimental Method to Determine the Thermal Time for Mixtures with a Positive Soret Coefficient}

For the case of mixtures with a positive Soret effect, the change of refractive index due to temperature and concentration variations occurs in the reverse to that in mixtures with a negative Soret effect. Application of the temperature gradient increases the maximum refractive index between walls; however, when separation occurs, the refractive index difference between the walls decreases. In other words, in a water/IPA mixture with a positive Soret effect, as IPA separates towards one wall, the refractive index difference between walls due to temperature differences in the system decreases during the thermodiffusion stage. Parts a and b of Figure 3-6 demonstrate the absolute value of the maximum refractive index variation between the hot and cold sides of the cell during all three stages of the thermodiffusion experiment for two mixtures with either a negative or positive Soret coefficient, respectively.

The experiment includes a $100 \mathrm{~s}$ soak at the mean of temperature $298.15 \mathrm{~K}$, then application of a temperature difference for $12 \mathrm{~h}$ (the thermodiffusion stage), and finally $6 \mathrm{~h}$ after terminating the applied temperature gradient in the system in order to return to the initial conditions. After the 
thermal time, the refractive index difference still increase for a negative Soret effect, while it decreased for the mixture with the positive Soret effect.

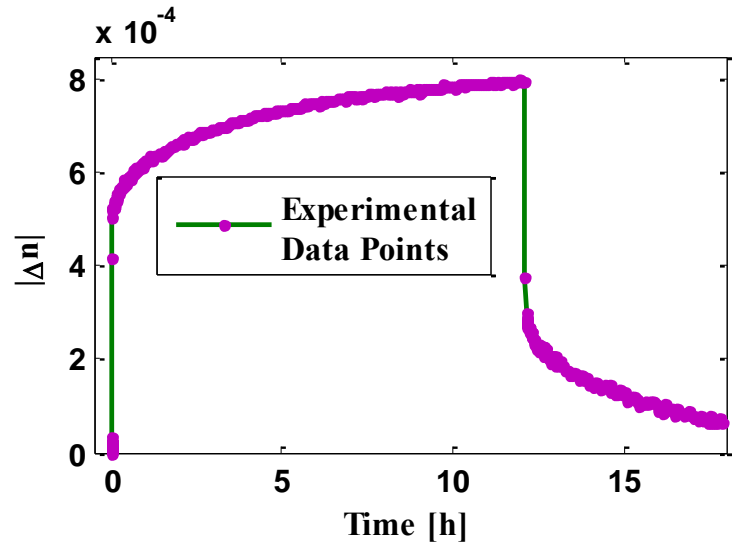

(a) : Negative Soret effect

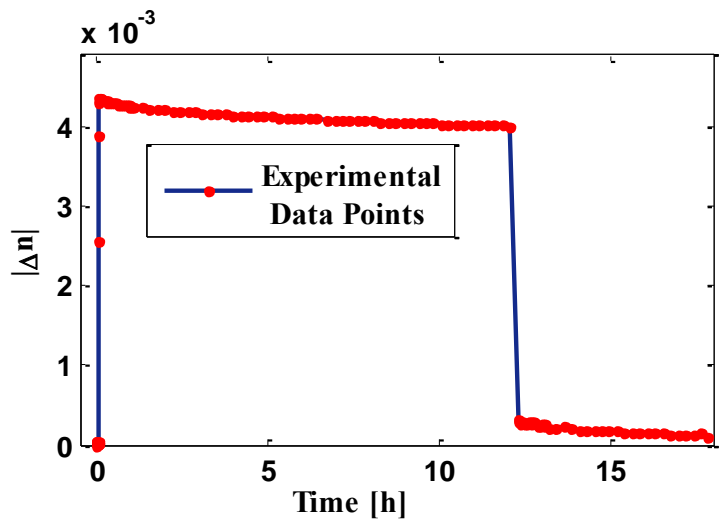

(b) Positive Soret effect during the entire experiment

Figure 3-6: Temporal maximum refractive index difference between the cold and hot walls Figure 3-7 schematically shows the variation of the refractive index due to the temperature difference between the hot and cold walls $\left(\Delta \mathrm{n}_{\mathrm{T}}\right.$, dashed line) and the refractive index difference caused by the concentration change at the beginning of thermodiffusion phase $\left(\Delta \mathrm{n}_{c}\right.$, dotted line).

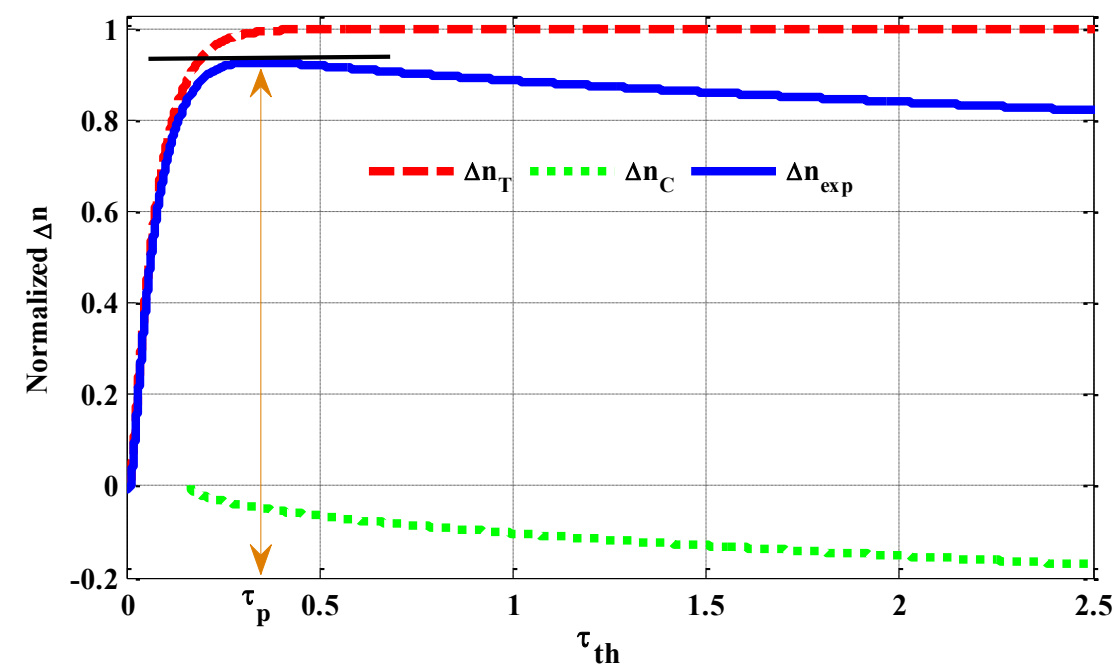

Figure 3-7: Temporal maximum refractive index difference between the cold and hot walls for positive Soret effect

The summation of these two refractive index differences $\left(\Delta \mathrm{n}_{\exp }=\Delta \mathrm{n}_{\mathrm{c}}+\Delta \mathrm{n}_{\mathrm{T}}\right)$ can be captured by the interferogram, which is shown Figure 3-7 (solid line). For mixtures with a positive Soret coefficient, it is not possible to precisely separate the effect of temperature and concentration 
variation during the thermal time, but it is evident that when the slope of the refractive index changes from positive to negative the refractive index variation depends mainly on the separation of components.

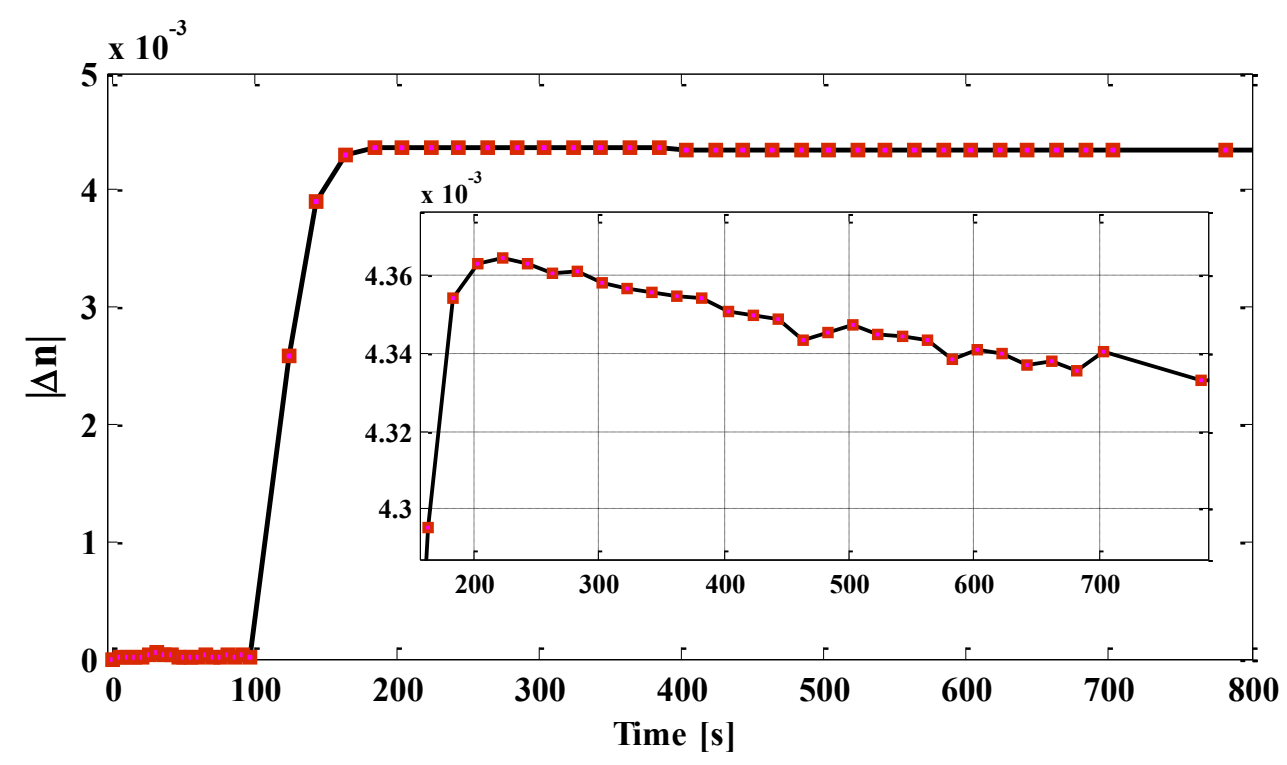

(a) the hot and cold walls

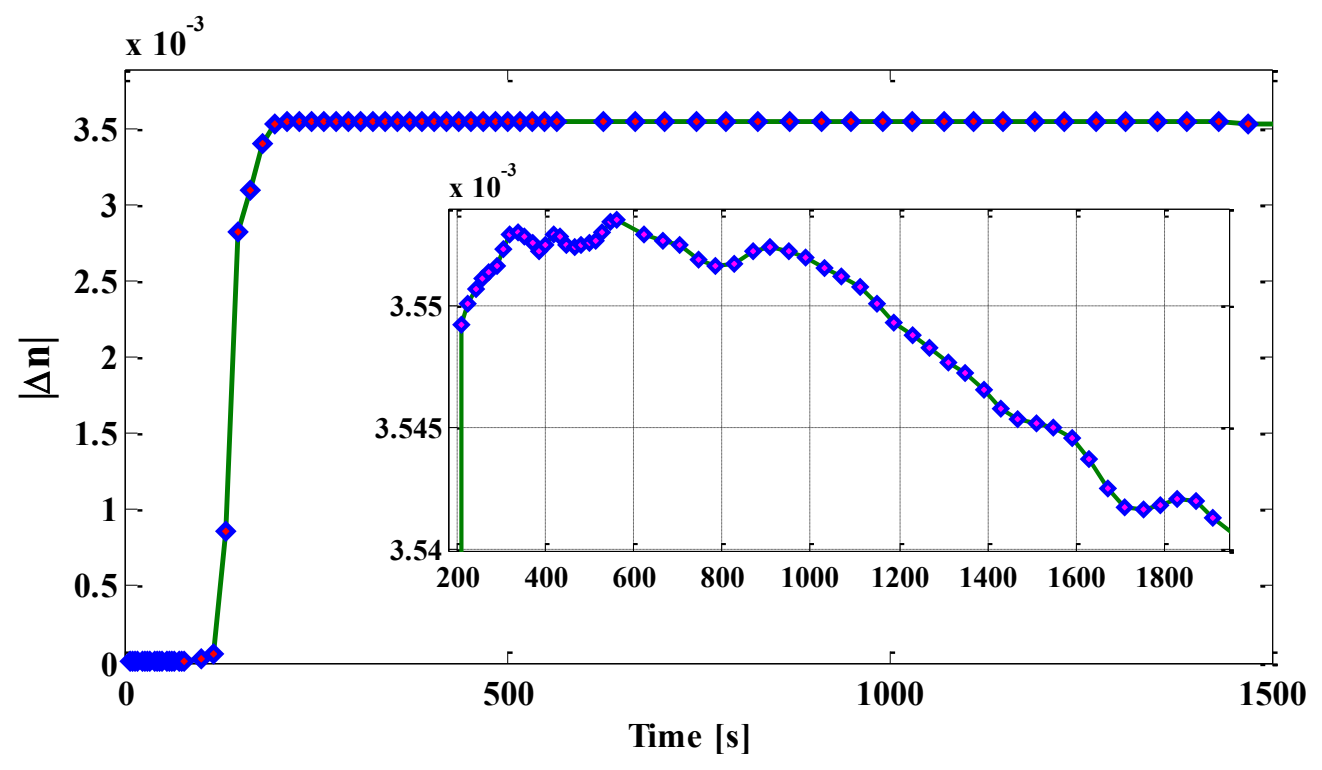

(b) $\mathrm{X}=1 / 4$ and $\mathrm{X}=3 / 4$ at the beginning of the experiment

Figure 3-8: Temporal maximum refractive index difference for the case with positive Soret between different locations 
In this experiment, while separation of the mixture components started before $\partial \mathrm{n} / \partial \mathrm{t}=0$, the time at which this is true might be the shortest answer for the thermal time of the mixture with a positive Soret coefficient (as thermodiffusion is coupling between heat and mass transfer). Furthermore, the effect of the temperature variation on the refractive index difference may exist after this point, but it would vary in the opposite direction to the effect of the concentration on refractive index and reduces the total measured $\Delta \mathrm{n}$ in the system. Thus, if we consider the reference image at the time when the maximum refractive index starts to decrease, the measured $\Delta \mathrm{n}$ after this point is not purely caused by the change in the concentration variation in the system. It can be claimed that the actual separation between the points $\mathrm{P}_{1}$ and $\mathrm{P}_{11}$ in Figure 3-1 should provide a greater value of $\Delta \mathrm{n}$ than is measured experimentally after this point. At this point, the separation has already started, and part of the influence of the mixture separation on the refractive index variation is cancelled out by the change of temperature in the cell.

Two correction values must be added to $\Delta \mathrm{C}$ to obtain the final result of the experiment $\left(\Delta \mathrm{C}_{\exp }\right)$, even if the thermal time is considered when the maximum $|\Delta \mathrm{n}|$ occurs. These corrections are modelled here. The curvature change of the refractive index variation between the walls occurred at different times for different height locations of the cell. The locations near the hot and cold walls reach thermal equilibrium faster than other locations, whereas locations with $X=1 / 4$ and 3/4 are the critical locations, which reach the steady-state thermal condition latest. Figure 3-8a illustrates the refractive index difference between points $\mathrm{P}_{1}$ and $\mathrm{P}_{11}$ (near cold hot walls) and part $\mathrm{b}$ of this figure demonstrates refractive index difference between the points at $x=1 / 4$ and 3/4.

Table 4: Optical properties of the water-IPA mixture measured at $\mathrm{T}=298 \mathrm{~K}$ for laser with the wavelength of

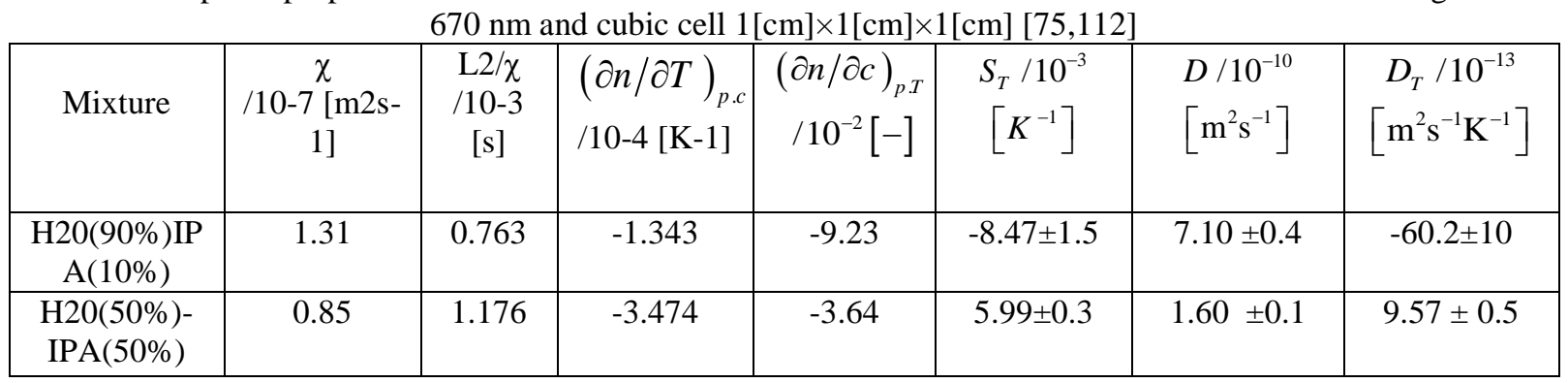

The maximum value of $\Delta \mathrm{n}$ occurred at $\mathrm{t} \sim 560 \mathrm{~s}$ for the locations that reached thermal equilibrium last, as shown in Figure 3-8b. This means that after 7.5 minutes of applying the temperature gradient, separation has begun (considering the first $100 \mathrm{~s}$ at a mean temperature of 
$298.15 \mathrm{~K})$. Note that $\mathrm{t}_{\mathrm{th}}=\mathrm{L}^{2} / \chi$ is about 20 minutes for a $50 / 50 \%$ by mass water/IPA mixture, which is the second mixture investigated in this study. This shows that for a mixture with a positive Soret effect, the maximum thermal time must not exceed more than $40 \%$ of $\tau_{\text {th }}$. It can be concluded that assuming the thermal time equal to $t_{t h}=L^{2} / \chi$ leads to an underestimation of $S_{T}$. It must be noted that the highest ratio of the separation occurs at the beginning of the thermodiffusion phase and must be measured precisely. Therefore, a correction value for this period must also be considered.

\subsubsection{Calculation of $\Delta C$ Correction Values for Mixtures With a Positive Soret Effect}

First, a correction value that might be considered due to the negative effect of temperature variation after the thermal time can be modelled. The exact time at which $\partial \mathrm{n}_{\exp } / \partial \mathrm{t}=0$ can be determined experimentally is $\mathrm{t}_{\mathrm{p}}$, as shown in Equation 3-19. As this $\Delta \mathrm{T}$ decreases the maximum refractive index variation between the walls, the corresponding correction value of $\Delta \mathrm{C}$ can be found as follows:

$\Delta C_{\text {Corr } 1}=\left(\Delta T_{t=t_{\infty}}-\Delta T_{t=t_{p}}\right) \frac{\left(\frac{\partial n}{\partial T}\right)}{\left(\frac{\partial n}{\partial C}\right)}$

This correction must be added to the maximum separation that is found close to the hot and cold walls as $\left(T_{(L, \infty)}-T_{(0, \infty)}\right)-\left(T_{\left(L, t_{p}\right)}-T_{\left(0, t_{p}\right)}\right)$. As the temperature close to the hot and cold walls $(\mathrm{X}=$ 0.01 and 0.99 ) must be considered, the $\Delta \mathrm{T}$ in Equation $3-23$ will be less than $2.5 \times 10^{-8} \mathrm{~K}$ or $\Delta \theta=2.5 \times 10^{-8}$. Using this value in Equation 3-19 results in the maximum possible value for $\Delta \mathrm{C}_{\text {corrl }}$, which is on the order of $10^{-6}$. Because the order of experimental separation is a least about $10^{-3}$, this correction value may not change the final result by more than $0.05 \%$. Thus, according to the experimental uncertainty this variation can be ignored.

The second correction value that must be considered due to separation during the thermal time can be modeled by adding in the experimental separation. Thus, Equation 3-24 can be derived. 


$$
\Delta n_{\exp }=\Delta n_{T}+\Delta n_{C} \Rightarrow \frac{\partial n_{\text {exp }}}{\partial t}=\frac{\partial n_{T}}{\partial t}+\frac{\partial n_{C}}{\partial t} \stackrel{\left.\frac{\partial n_{\text {exp }}}{\partial t}\right|_{t=p_{p}} ^{=0}}{\longrightarrow} \frac{\partial n_{T}}{\partial t}=-\frac{\partial n_{C}}{\partial t}
$$

As the temperature variation in the cell is known, $\partial \mathrm{T} / \partial \mathrm{t}$ and the values of $\partial \mathrm{n}_{\mathrm{T}} / \partial \mathrm{t}$ and $\partial \mathrm{n}_{\mathrm{c}} / \partial \mathrm{t}$ at $\mathrm{t}_{\mathrm{p}}$ can be calculated. According to the measured $\Delta C_{\text {exp }}$, the initial value of $S_{T}$ can be found. As we know, the final corrected value of $S_{\mathrm{T}}$ may not change more than 5 to $10 \%$, so this value can be used and based on this equation $\partial \mathrm{n}_{\mathrm{c}} / \partial \mathrm{t}$ can be calculated analytically. As the value of $\partial \mathrm{n}_{\mathrm{c}} / \partial \mathrm{t}$ at $\mathrm{t}_{\mathrm{p}}$ is known, we can estimate the time corresponding to this value. Having this neglected diffusion time and employing the derived equation yields the value of $\Delta \mathrm{C}_{\text {corr2 }}$. Thus, the total correction $\Delta \mathrm{C}$ during the experiment can be expressed as Equation 3-25.

$$
\Delta C=\Delta C_{\text {exp }}+\Delta C_{c o r r 1}+\Delta C_{c o r r 2}
$$

For our test mixture of 50/50 water/IPA, application of the mentioned procedure yielded an increment of $4 \%$ for the maximum separation in the system.

\subsubsection{Implementation of an Iterative Evolutionary Optimization Curve-fitting Technique to Calculate $S_{T}$}

In this section, an iterative curve-fitting method based on an evolutionary optimization algorithm is described for the MZI thermodiffusion experiments used to accurately measure the Soret coefficient. This technique is useful even when the precise thermal time could not be calculated according to the processes in the previous two sections. In these cases, the thermal time is calculated based on $\mathrm{L}^{2} / \chi$, and as a result of having some separation occurring before the thermal time ends, a correction procedure must be applied to extract the real value of the separation.

Table 5: Result of Iterative method after various iterations

\begin{tabular}{|c|c|c|c|c|c|c|c|}
\hline Iteration\# & 1 & 2 & 3 & 4 & 5 & 6 & 10 \\
\hline$\Delta \mathrm{C} / 10-3[-]$ & 2.74 & 2.93 & 2.96 & 2.99 & 3.01 & 3.04 & $3.05 \pm 0.02$ \\
\hline $\mathrm{ST} / 10-3[\mathrm{~K}-1]$ & 7.45 & 8.21 & 8.35 & 8.49 & 8.58 & 8.65 & $8.65 \pm 0.21$ \\
\hline
\end{tabular}

All of the above-mentioned procedures were used to estimate the $S_{\mathrm{T}}$ and $\mathrm{D}$ for the separation that was captured after $\mathrm{t}=\mathrm{t}_{\mathrm{th}}$. As mentioned earlier, we assumed the separation started when $\mathrm{t}=$ $0.221 \times \mathrm{t}_{\mathrm{th}}$. This means that the separation between $\mathrm{t}=0.221 \times \mathrm{t}_{\mathrm{th}}$ and $\mathrm{t}=\mathrm{t}_{\mathrm{th}}$ was not considered in the final results. Then using Equation 3-25 and the first estimates of the $S_{\mathrm{T}}$ and $\mathrm{D}$, the separation 
after $\mathrm{t}=0.779 \times \mathrm{t}_{\mathrm{th}}$ was found. Next, $\left.\Delta \mathrm{C}\right|_{\mathrm{t}_{=0.779 \times} \mathrm{t}_{\mathrm{th}}}$ was added to all measured $\Delta \mathrm{C}_{\text {exp }}$ to adjust for the separation that started at $\mathrm{t}=0.221 \times \mathrm{t}_{\mathrm{th}}$. Up to this point, the first iteration of the experimental result correction has been completed. For the next iterations, we used the same optimization method to find the $S_{T}$ and D based on the corrected concentration difference between walls. Then according to the new estimated coefficients, a new value for $\left.\Delta \mathrm{C}\right|_{\mathrm{t}_{=0.79 \times \times t h}}$ was introduced and this procedure was iterated until a steady-state solution was reached.

Table 6: Summarized thermal time and Soret coefficient calculated using different methods in comparison with assuming $\mathrm{t}_{\mathrm{th}}=\mathrm{L}^{2} / \chi$ and benchmark value of Soret coefficient

\begin{tabular}{|c|c|c|c|c|c|c|}
\hline \multirow{2}{*}{ Mixture } & & $\begin{array}{c}\mathrm{ST} \text { assuming } \\
\text { th= } \mathrm{L}^{2} / \chi\end{array}$ & $\begin{array}{c}\text { Method for } \\
\text { Negative } \mathrm{S}_{\mathrm{T}}\end{array}$ & $\begin{array}{c}\text { Method for } \\
\text { positive } \mathrm{S}_{\mathrm{T}}\end{array}$ & $\begin{array}{c}\text { Iterative } \\
\text { method }\end{array}$ & $\begin{array}{c}\text { Benchmark } \\
\text { Value[75] }\end{array}$ \\
\hline H20(90\%)- & Thermal time [s] & 763 & 216 & --- & 763 & --- \\
\cline { 2 - 7 } IPA(10\%) & Soret coefficient [1/K] & $-7.45 \pm 0.21$ & $8.56 \pm 0.21$ & --- & $-8.65 \pm 0.2$ & $-8.47 \pm 1.5$ \\
\hline H20(50\%)- & Thermal time [s] & 1176 & --- & 481 & 1176 & --- \\
\cline { 2 - 7 } IPA(50\%) & Soret coefficient [1/K] & $5.18 \pm 0.29$ & --- & $5.86 \pm 0.29$ & $5.91 \pm 0.3$ & $5.99 \pm 1.5$ \\
\hline
\end{tabular}

It must be noticed that in the first iteration in this method we assumed that the separation of the component starts at $\mathrm{t}_{\mathrm{c} 0}=\mathrm{t}_{\mathrm{th}}$; however, for the next iterations we corrected this assumption by using $\mathrm{t}_{\mathrm{c} 0}=0.221 \times \mathrm{t}_{\mathrm{th}}$ as the initial time for the separation. Afterwards, the GA optimization approach using the corrected time and separation was implemented to extract a more accurate result. A steady value with less than 0.001 variations was obtained after 5 to 10 iterations for our cases in this study. Table 5 demonstrates the results of this iterative method after various iterations for the mixture with a negative Soret effect; however, this method can be implemented for mixtures with a positive Soret effect, or even for multicomponent mixtures. A convergence of the Soret coefficient value after 6 iterations is seen in Table 5. It appears that applying this method may improve the results by about $10 \%$. Moreover it must be noted that the first iteration generated the result by considering $\mathrm{t}_{\mathrm{th}}=\mathrm{L}^{2} / \chi$. Comparing the results in Table 5 with the methods described above, we have shown that performing two iterations calculates about $90 \%$ of the correction to the Soret coefficient value.

The results of these different techniques that were employed in this investigation are summarized in Table 6 and are compared with the benchmark value of the mixtures, obtained from Mialdun et al. [75]. When $t_{t h}=L^{2} / \chi$, the iterative method improved the final Soret coefficients by about 10 $\%$ for mixtures with either negative or positive Soret effects. This suggests that assuming a 
thermal time equal to $\mathrm{L}^{2} / \chi$ causes an underestimation of the Soret coefficient and the maximum separation value. The final Soret coefficients for the mixture with a negative Soret effect calculated using the iteration method and the analytical method were quite similar with only about $1 \%$ variation between them. In addition, these calculated values show a good agreement with the benchmark value, with less than $2 \%$ variation. The results are similar for mixtures with a positive Soret effect; however, for mixtures with a positive Soret effect, instead of a theoretical method to calculate the thermal time for this experiment an aided experimental method is used. For both types of mixtures, we showed that the precise thermal time for Mach-Zehnder interferometry must not exceed more than $40 \%$ of L ${ }^{2} / \chi$. It must be noted that in previous studies the mentioned ratio $\left(\mathrm{L}^{2} / \chi\right)$ was considered as the thermal time, which we have now shown likely underestimates the final result of the thermodiffusion experiment $[65,66,109,110]$.

\subsection{Conclusions}

In this study, a theoretical approach for the measurement of the negative Soret coefficients and an aided experimental model for the measurement of positive Soret coefficients are introduced to estimate the thermal time during measurements of the Soret coefficients using optical MachZehnder interferometry. A new formulation to calculate the thermal relaxation time for the Soret effect experiment by means of Mach-Zehnder interferometry was proposed for the negative Soret effect, and an experimental approach was provided for measurement in the case of the positive Soret effect. For both negative and positive Soret effects, it was demonstrated that the precise thermal time for MZI must not exceed more than $40 \%$ of $\mathrm{L}^{2} / \chi$. The measured Soret coefficients were in good agreement with benchmark values; all of them possessed less than a $3 \%$ variation. It was proved that assuming thermal time equal to $\mathrm{L}^{2} / \chi$ causes about $10 \%$ underestimation in the measurement of the Soret coefficients and maximum separation values. We also introduced an iterative optimization method to correct the final values of the Soret coefficient and the maximum separation when $\mathrm{L}^{2} / \chi$ is assumed as the thermal time for MZI thermodiffusion experiments. The iterative method improved the final Soret coefficients about $9 \%$ for both negative and positive Soret effects. 


\section{Chapter 4: Detail Heat Transfer Analysis using MZI for Two Different Cell Configurations}

\subsection{Introduction}

Temperature measurement in an apparatus can be performed using different techniques. Two of the most widely used are optical measurements, such as refractive index measurement and interferometry, and thermocouple measurements. Both types of techniques have their advantages and disadvantages, which makes them useful in various applications. For instance, optical techniques have been used as a non-contact method [121], while thermocouple devices have been used for measuring the surface temperature of a liquid metal $[122,123]$. A combination of both techniques was employed by the selectable optical diagnostics instrument (SODI) apparatus on board the International Space Station (ISS). The typical geometry and boundary conditions of the sample cells for two different series' of experiments on board the ISS are illustrated in Figure 4-1. The temperature gradient was controlled by a thermal unit that consisted of thermal sensors, Peltier elements, and a computer controller unit.

\subsection{Chapter Aims}

In this chapter, we investigated the heat transfer and temperature measurement during thermodiffusion experiments using the Selectable Optical Diagnostics Instrument (SODI) apparatus at reduced gravity. The principles of Mach Zehnder Interferometry (MZI) were used for the measurement of various parameters, such as temperature and concentration, in transparent cells in the SODI apparatus on board the International Space Station (ISS). In particular, the critical behavior of heat flux at the cell's boundaries was studied. We discuss deviations between the temperature differences at the hot and cold sides of the cell when measured using optical image processing and Peltier elements, and we suggest a reason for this discrepancy. According to the extent of this experimental method, we provide the necessary adjustments to have accurate post-processing for MZI thermodiffusion experiments. 


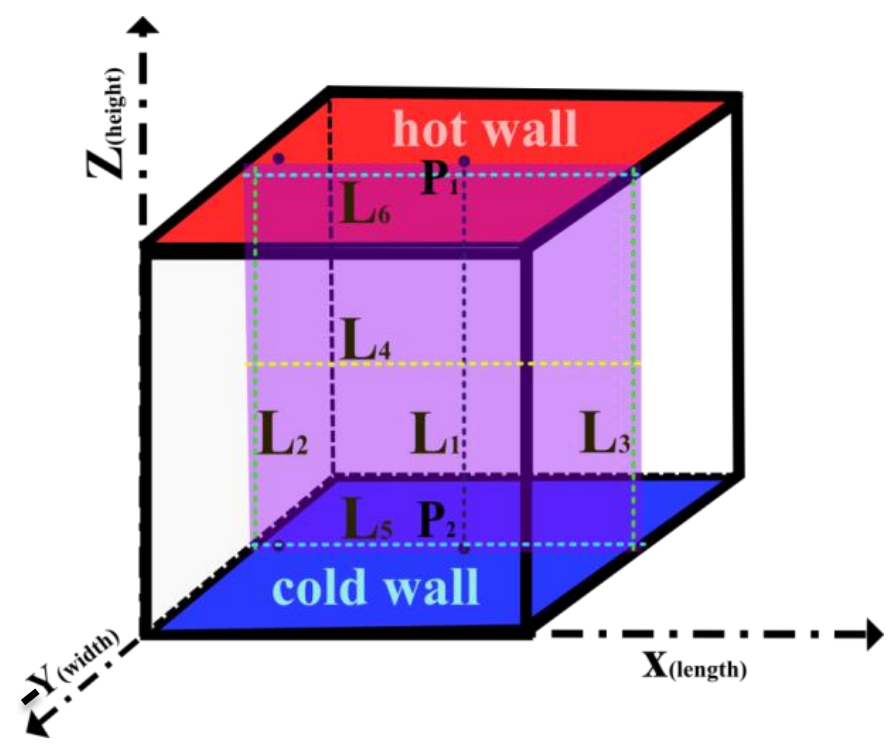

Figure 4-1: Geometry of the SODI cell.

The quality and accuracy of the thermal controller unit of the SODI apparatus were also studied. The sensitivity of the temperature measurements using interferometry was carried out, and we found good performance of this method in a microgravity environment. The effects both of the weak insulation at the walls and of the distance between the hot and cold sides of the cell are discussed. While the thermal unit of the SODI apparatus controlled the temperature across the cell within an acceptable accuracy range for all of the thermodiffusion experiments, marginal differences between various SODI experiments were observed and reported in this study. Finally, we discuss the impacts of different measurement techniques for the temperatures in the thermodiffusion experiment. Comparing the results of this study with literature shows that the enhancement of heat transfer analysis can lead to a more accurate measurement of the Soret coefficient. We have analyzed the heat transfer inside the two cells (one having a cube shape and the other one a rectangular shape) using the recorded optical data. We have particularly focused on the regions close to the walls, since this is the location that has been previously shown to possess the greatest imperfections in thermal gradients due to unintended heat loss from the sample chamber [27,112]. We explain the source of disagreement between the optical measurement of the temperature inside the cells and the temperature measured using the thermal sensors at both sides of the cells. First, a brief description of the SODI apparatus is given, and then an introduction to the principles of the interferometry experiment to study heat and mass transfer inside the cell is provided. 
Table 7: Properties of the water-IPA (90:10\%wt) mixture measured at $\mathrm{T}=298 \mathrm{~K}$ for case 1 IVIDIL Run $2 \mathrm{R}$ [75]

\begin{tabular}{|c|c|c|c|c|}
\hline$(\partial n / \partial T)_{p . c}$ & $(\partial n / \partial c)_{p . T}$ & $S_{T} \times 10^{-2}$ & $D / 10^{-10}$ & $D_{T} / 10^{-13}$ \\
$/ 10^{-4}\left[\mathrm{~K}^{-1}\right]$ & $/ 10^{-2}[-]$ & {$\left[K^{-1}\right]$} & {$\left[\mathrm{m}^{2} \mathrm{~s}^{-1}\right]$} & {$\left[\mathrm{m}^{2} \mathrm{~s}^{-1} \mathrm{~K}^{-1}\right]$} \\
\hline-1.3427 & -9.2305 & -8.47 & 7.11 & -6.01 \\
\hline
\end{tabular}

Next, the experimental setup and procedure for this experiment are explained. The results are then presented and discussed. Finally, the advantages and disadvantages of each of the methods in sensitive coupling heat and mass transfer problems are covered, and the effect of using different experimental techniques to measure the Soret coefficient is discussed. The most reliable temperature measurement method to process the MZI images for coupled heat and mass transfer experiments also introduced based on a benchmark value of Soret coefficient $\left(S_{T}\right)$.

Table 8: Optical properties of the THN-IBB-C12 (10:80:10\%wt) mixture measured at T=298 K for case 2

\begin{tabular}{|c|c|c|c|c|c|}
\hline $\begin{array}{l}\text { Laser } \\
\text { Type }\end{array}$ & $\begin{array}{c}\lambda \\
{[\mathrm{nm}]}\end{array}$ & $\begin{array}{c}(\partial n / \partial T)_{p, I B B, T H N} \\
/ 10^{-4}\left[\mathrm{~K}^{-1}\right]\end{array}$ & $\begin{array}{c}\left(\partial n / \partial c_{C_{12}}\right)_{p, T \text { THN }} \\
/ 10^{-2}[-]\end{array}$ & $\begin{array}{c}\left(\partial n / \partial c_{T H N}\right)_{p, T, I B B} \\
/ 10^{-1}[-]\end{array}$ & $\begin{array}{c}\left(\partial n / \partial c_{I B B}\right)_{p, T, C_{12}} \\
/ 10^{-2}[-]\end{array}$ \\
\hline MR & 670 & -4.812 & -5.72 & 1.213 & -7.57 \\
\hline MN & 935 & -4.763 & -5.38 & 1.240 & -6.96 \\
\hline
\end{tabular}

\subsection{Experimental Apparatus and Procedure}

The first series of the SODI experiments, IVIDIL, started in late 2009 to study the effects of temperature and vibration on thermodiffusion in two binary associating mixtures of water and isopropanol. The aim of the DSC facilities, started in 2010, was to study the Soret effect in hydrocarbon ternary mixtures. The physical and optical properties of the test mixtures at the mean temperature of $298.15 \mathrm{~K}$ are provided in Table 7 and Table 8 for the binary and the ternary experiments, respectively. It must be noted that this includes cases with no applied vibration.

\subsubsection{Experimental Procedure}

Each experimental case was performed in three stages: half an hour at $298.15 \mathrm{~K}$ (the mean temperature), several hours in the presence of a thermal gradient (thermodiffusion phase), and the diffusion phase in which the mixture was brought to a homogeneous state by removing the applied thermal gradient. Here, we focus on the experimental time from the first stage to the beginning of the second stage. 
We monitored the refractive index variation inside the cell at different time ranges: at the beginning of the experiment where there is an absence of the thermal gradient and also during the thermal time (for binary associating mixtures $t_{\text {th }}<770 \mathrm{~s}$ and for ternary non-associating mixtures $t_{t h}<330$ ) of the experiment. In this study, two experimental cases were investigated in detail: case 1 (Run2R according to the timetable) and case 2 (Run12 in the DSC timetable), which possessed temperature gradients of $5 \mathrm{~K}$ and $10 \mathrm{~K}$, respectively. Case 1 is a binary mixture of water $90 \%$, and isopropanol $10 \%$ and case 2 is a ternary mixture of $80 \%$ isobutylbenzene, 10 $\%$ tetrahydronaphthalene, and $10 \%$ dodecane by mass.

\subsection{Results and Discussion}

In this study, several parameters such as the temperature profile, the heat transfer rates near the cavity walls, and the maximum temperature difference in the cells were investigated. The role of the temperature controller is to provide a constant and stable temperature difference in order to measure the Soret coefficients of the mixture. The absolute value of the temperature difference affects the Soret coefficient calculation as shown below. This equation represents the analytical solution of the Soret separation in the binary mixtures at zero gravity, which is a purely thermodiffusion-driven process [120].

$$
\Delta C(t)=C(t, L)-C(t, 0)=-S_{T} C_{0}\left(1-C_{0}\right) \Delta T\left[1-\frac{8}{\pi^{2}} \sum_{n, \text { odd }}^{\infty} \frac{1}{n^{2}} \exp \left(-n^{2} \frac{t}{\tau_{D}}\right)\right]
$$

In the above equation, $\tau_{\mathrm{D}}$ is the diffusion time, which has been measured to be about $25 \mathrm{~h}$ for water/IPA mixtures and $6 \mathrm{~h}$ for hydrocarbon mixtures. As noted, the above equation can be used to calculate the Soret coefficient $\left(\mathrm{S}_{\mathrm{T}}\right)$ from the experimentally measured component separation. In fact, the left hand side of the equation, which is the maximum separation defined as the average change in the concentration of the species at the heated wall, was obtained experimentally, and the above equation was employed to find the best fit to the experimental data using the best values of $S_{T}$ and/or $\tau_{\mathrm{D}}$. Using this method, the Soret coefficient was calculated experimentally based on the temperature difference measured across the cell at the inner boundaries. Furthermore, due to the cropping process, the height of the area that was analyzed is slightly smaller than the real dimensions of the cell. 


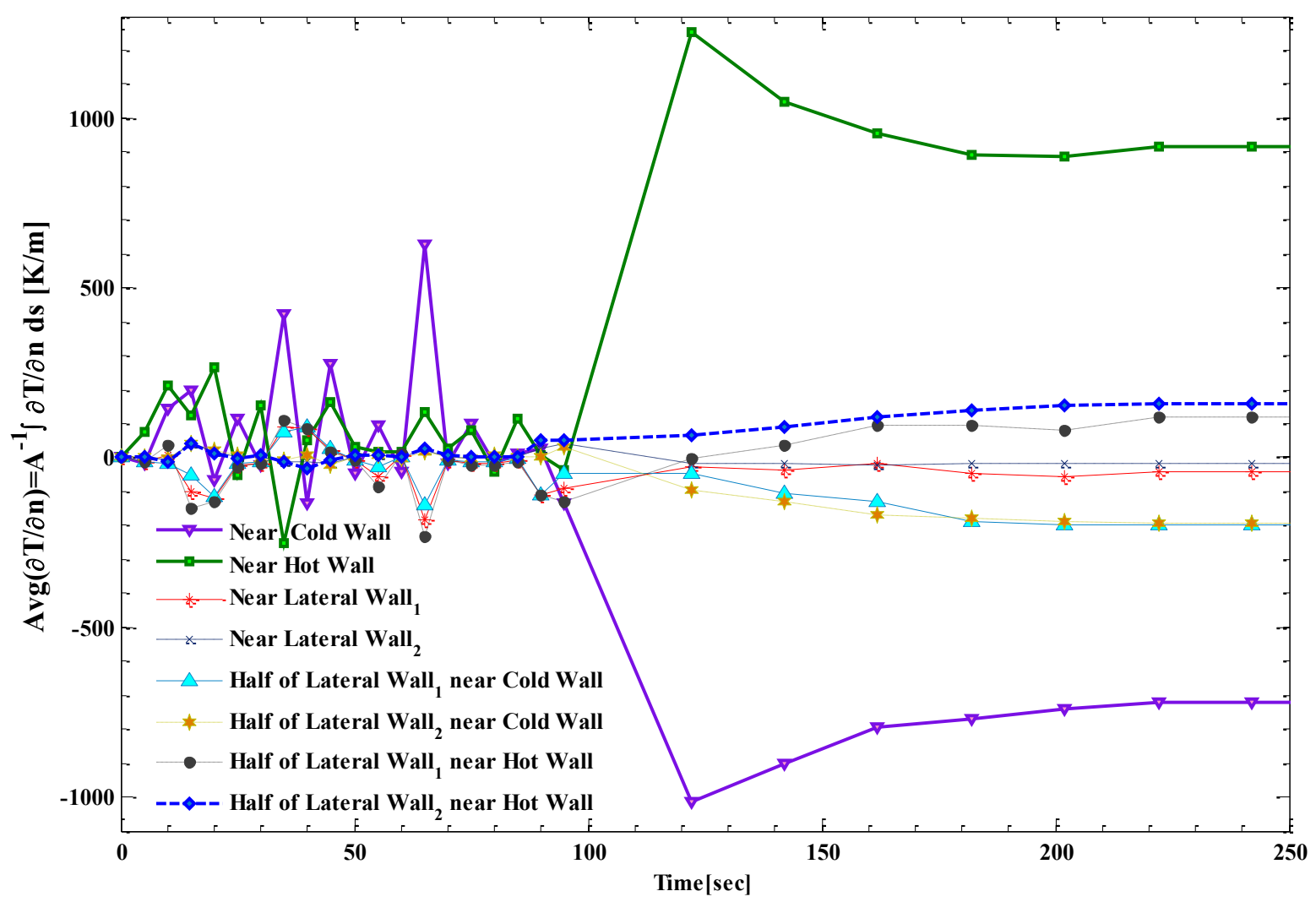

Figure 4-2: Transient variation of mean temperature gradient normal to the boundaries of the case 1 (IVIDIL-Run2R).

Thus, the temperature difference in the cropped area was evaluated and used in the calculations. The error between the temperature difference measured by the thermocouples and that reported by the Peltier elements directly affected the final values of Soret coefficient.

For instance, for the case that the thermal sensors showed a $5.00 \mathrm{~K}$ temperature difference between the hot and cold sides, it was measured optically at $4.45 \mathrm{~K}$ due to the cropping of the images. We should keep in mind that the temperature contrast factor does not change more than one percent from the mean temperature of $293.15 \mathrm{~K}$ to $303.15 \mathrm{~K} \mathrm{[124]}$ and that the maximum error in the contrast factor at the mean temperature of $298.15 \mathrm{~K}$ shows less than a $3 \%$ variation for different mixtures [125,126]. However, there is a difference of more than $11 \%$ between the recorded temperature using the thermal sensors and that obtained by optical methods. Because of the high accuracy of this optical method [125,126], we relied on the it over the sensors for the 
temperature difference used in the calculation of the Soret coefficient [27,112]. It must be noted that the effect of the materials in the cell itself on this measurement is negligible, since the temperature contrast factor of quartz is more than one order of magnitude smaller than the liquid contrast factors.

As indicated in Figure 4-1, the temperature variation and the rate of temperature change along lines $\mathrm{L}_{1}$ to $\mathrm{L}_{6}$ were studied. In addition, the temperature field was measured inside the illustrated plane in Figure 4-1. Moreover, for case 1 (IVIDIL case) with the presence of the second optical side view of the cell, temperature variation inside the additional plane (parallel to yz-plane in Figure 4-1) was also monitored. Eventually, the maximum temperature difference between $\mathrm{P}_{1}$ and $\mathrm{P}_{2}$ was also compared to the data measured by the thermocouples.

\subsubsection{Thermal Analysis of the IVIDIL Cell}

In this section, we discuss the thermal performance of the SODI cells during the $100 \mathrm{~s}$ before application of the temperature gradient (at a mean temperature of $298.15 \mathrm{~K}$ ) and then during the first $150 \mathrm{~s}$ of the thermal time (770 s). For that purpose, we have obtained the thermal gradient of each point on the boundaries from the processed data. The derivative of the temperature variation along the normal direction of each wall of the cell was calculated using a forward finite difference scheme as shown in below:

$\left.\frac{\partial T(x, y)}{\partial x}\right|_{x_{0}}=\frac{-T\left(x_{0}+4 \Delta x, y\right)+8 T\left(x_{0}+3 \Delta x, y\right)-8 T\left(x_{0}+\Delta x, y\right)+T\left(x_{0}, y\right)}{12 \Delta x}+\left.\frac{\Delta x^{4}}{30} \frac{\partial^{5} T(x, y)}{\partial x^{5}}\right|_{x_{0}}$

This equation gives the first derivative using four consecutive data points and has an approximate error on the order of $\Delta \mathrm{x}^{4}$ in which $\Delta \mathrm{x}$ is the length of each pixel. It must be noted that the first derivative using two or three consecutive data points has the higher errors on the order of $\Delta \mathrm{x}^{2}$ and $\Delta \mathrm{x}^{3}$. Consequently, we decided to calculate the first derivative using equation 42. Due to the resolution of the experiment and cropping size (700 pixels were monitored along each direction representing $9.5 \mathrm{~mm}$ each of the height and the length of the cell) each pixel was about $12.9 \mu \mathrm{m}$ in length. Figure 4-2 illustrates the average value of the thermal gradient versus time close to the walls of the cell for case 1 (assigned $\Delta \mathrm{T}=5 \mathrm{~K}$ ). The temperature difference was applied to the system at $\mathrm{t}=100 \mathrm{~s}$. 


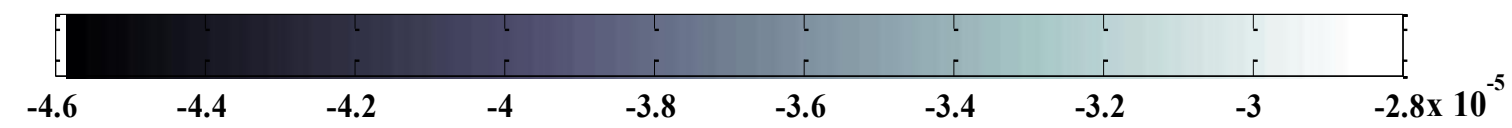

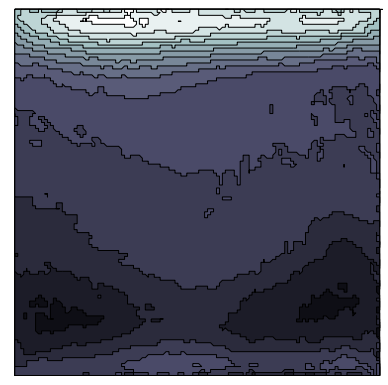

(a)

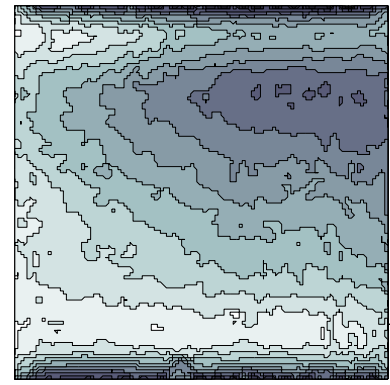

(d)

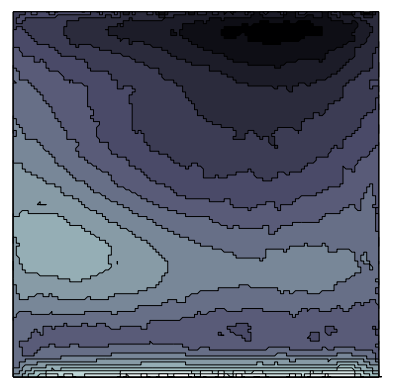

(g)

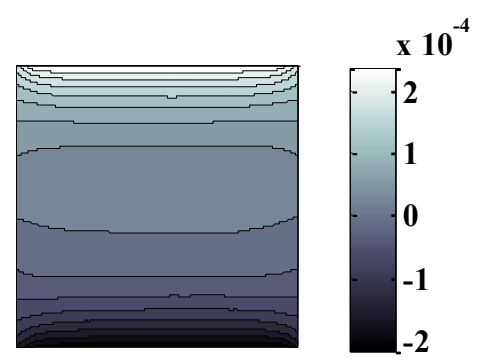

(j)

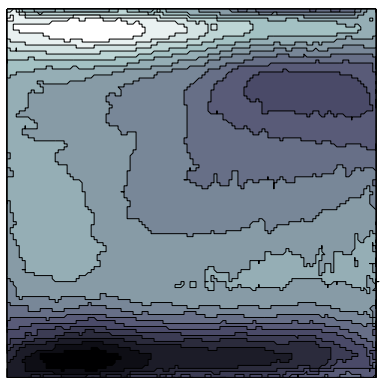

(b)

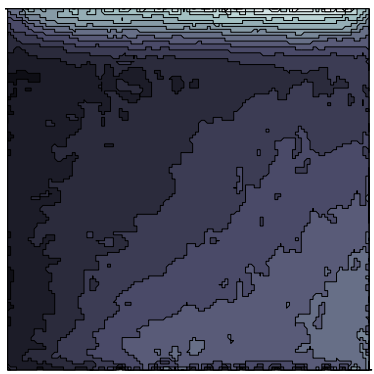

(e)

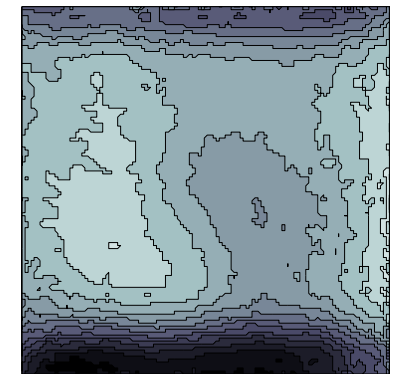

(h)

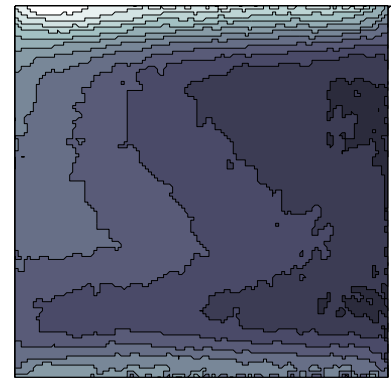

(c)

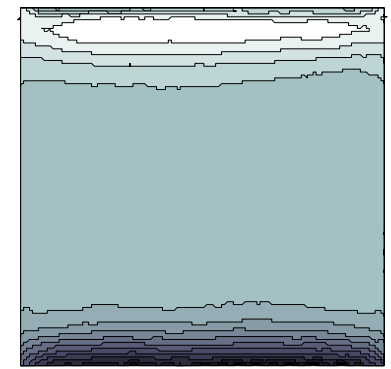

(f)

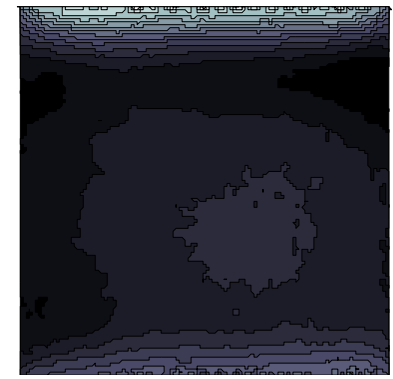

(i)

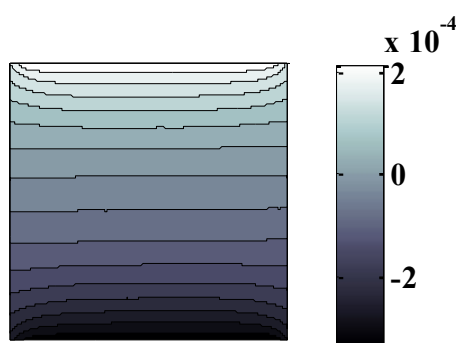

(k)

Figure 4-3: Refractive index of the front view of binary cell case 1 at (a to i) before applying temperature gradient $0<\mathrm{t}<100 \mathrm{~s},(\mathrm{j})$ at $\mathrm{t}=220 \mathrm{~s}$ and $(\mathrm{k})$ after the thermal time $(\mathrm{t}=770 \mathrm{~s})$ 


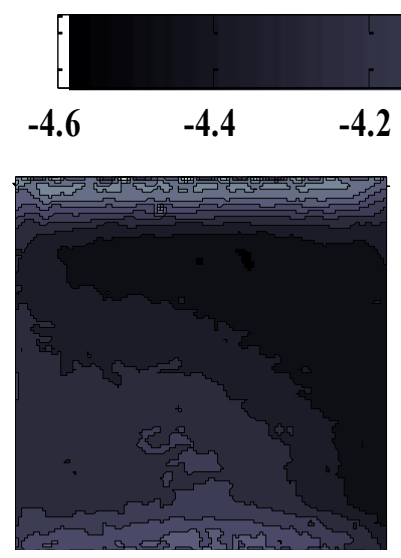

(a)

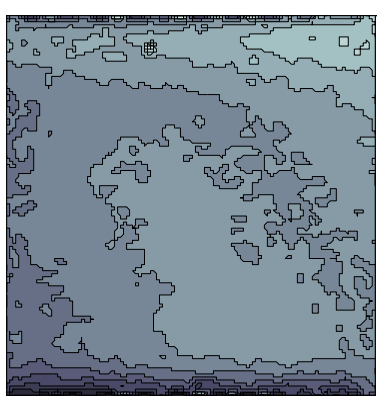

(d)

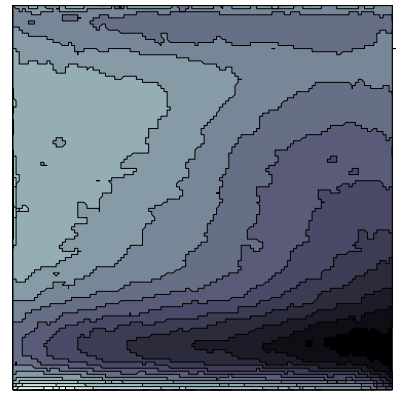

(g)

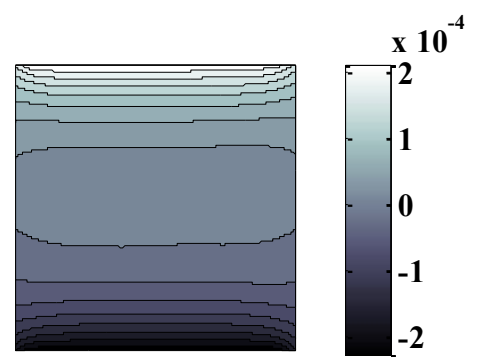

(j)

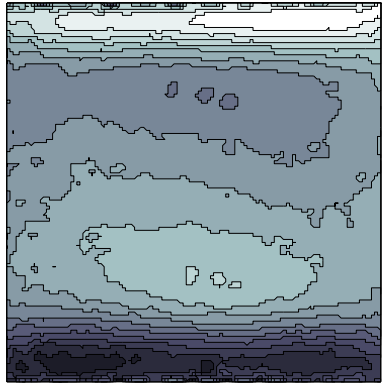

(b)

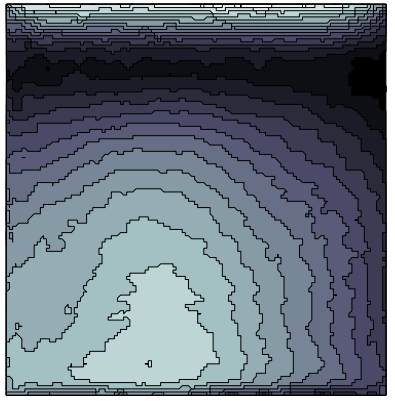

(e)

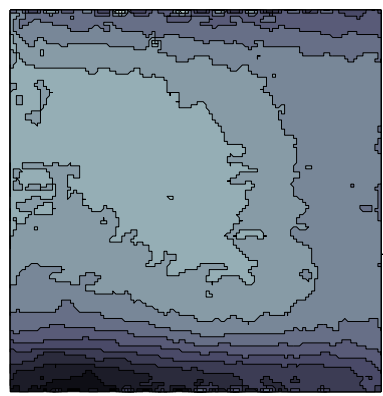

(h)

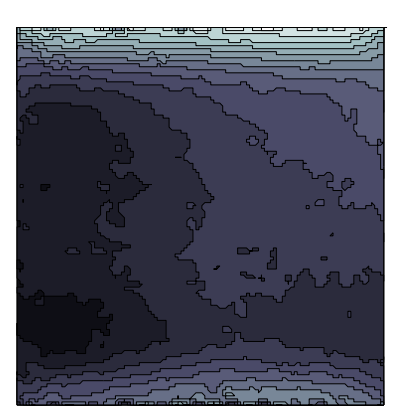

(c)

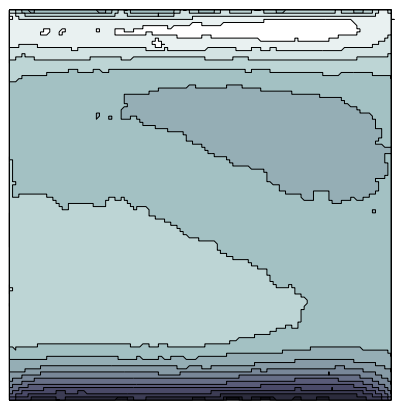

(f)

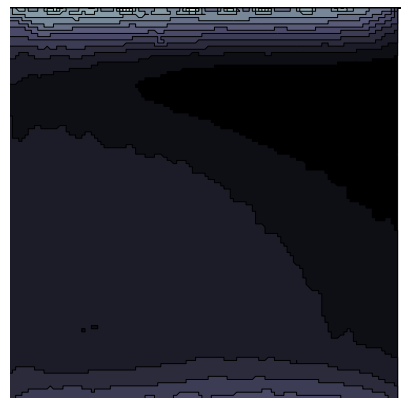

(i)

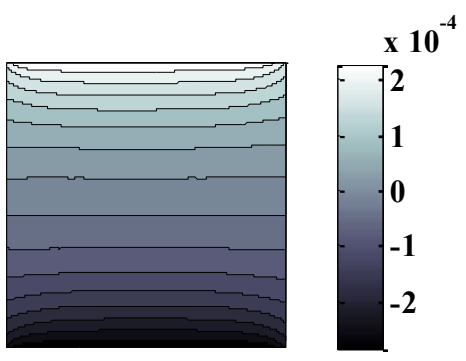

(k)

Figure 4-4: Refractive index of the side view of the binary cell case 1 at (a to $i$ before applying temperature gradient $0<\mathrm{t}<100 \mathrm{~s},(\mathrm{j})$ : at $\mathrm{t}=220 \mathrm{~s}$ and $(\mathrm{k})$ after the thermal time $(\mathrm{t}=770 \mathrm{~s})$ 
After 2 minutes of application of the heat to the system, a steady temperature gradient of $\sim 700$ $\mathrm{K} / \mathrm{m}$ was achieved in the system. It must be noted that this steady value for both the hot and cold walls was more than the predicted value; according to the theoretical heat transfer (reaching the linear variation of the temperature between hot and cold sides at steady state) in the liquid, the predicted value should be around $500 \mathrm{~K} / \mathrm{m}$. The extreme values are evident at the first data point after applying thermal gradient $(t=122 \mathrm{~s})$. After this point, the temperature gradient decreases to the steady state value at $(\mathrm{t}=250 \mathrm{~s})$. It is worth mentioning that controller overshoot is noticeable at $\mathrm{t}=170 \mathrm{~s}$. The average heat transfer close to the two lateral walls showed an approximately zero average flux along those walls, $q=k \frac{d T}{d x} \sim 0$ (dashed cross and solid star lines in Figure 4-2). These walls are meant to insulate the cell from lateral heat transfer. Although the value of average the temperature gradient was zero, it can be shown that there was a considerable leakage of heat through these walls. When the thermal gradient was taken for the half of the lateral walls close to either the heat or cold source, we found that not only was the heat transfer close to lateral walls not zero, but also that it had a value of $\sim 200 \mathrm{~K} / \mathrm{m}$ for each of the walls. It follows, then, that ideal insulation was not obtained around the cell, while the summation of the negative and the positive heat loses along lateral is zero. In fact, the heat was dissipated to the external environmental from the half of the lateral walls close to the hot wall (a positive thermal gradient value) and opposite to the direction of heat flux that was seen near to the cold side. Thus, the summation of the heat dissipation along each one of the lateral walls is zero. These heat losses at the boundaries may affect the thermodiffusion process close to the lateral walls, thus, this effect was considered during the processing of thermodiffusion experiments.

We also observed strong fluctuations in the average temperature gradient on the walls during the initial isothermal stage $(\Delta \mathrm{T}=0)$. Since copper blocks are attached to two sides of the cell, it is reasonable to have stronger fluctuations close to the hot and cold sides. The thermal controller reduces the magnitude of these temperature fluctuations as time passes towards the steady state. As shown in Figure 4-2, these fluctuations occurred at $t=60 \mathrm{~s}$. In addition, the strong fluctuation close to the hot and cold walls resulted in noticeable fluctuations close to the lateral walls at $\mathrm{t}=$ 15,35 , and $65 \mathrm{~s}$. 


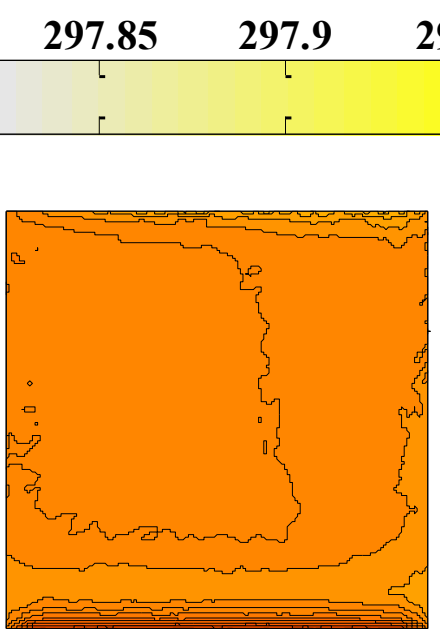

(a.1) $\mathrm{t}=25 \mathrm{~s}$

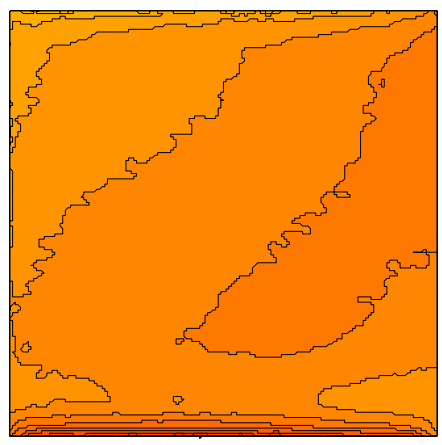

(a.2) $\mathrm{t}=25 \mathrm{~s}$

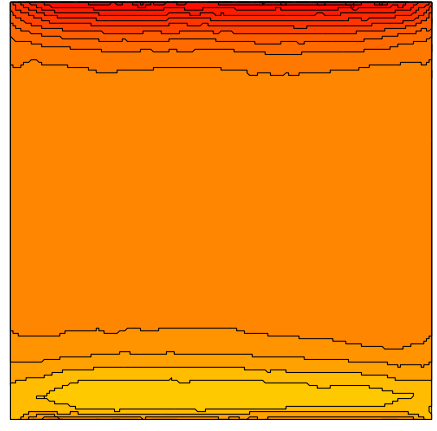

(b.1) $\mathrm{t}=65 \mathrm{~s}$

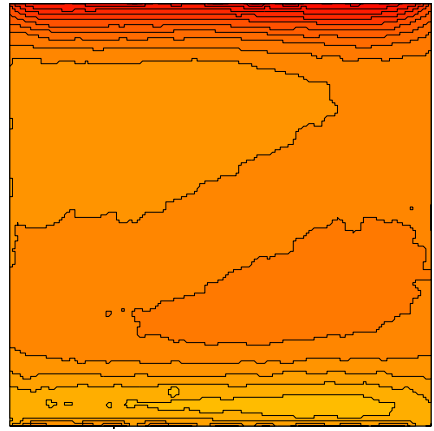

(b.2) $\mathrm{t}=65 \mathrm{~s}$

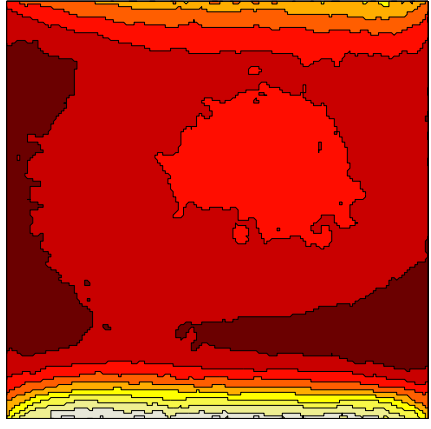

(c.1) $\mathrm{t}=90 \mathrm{~s}$

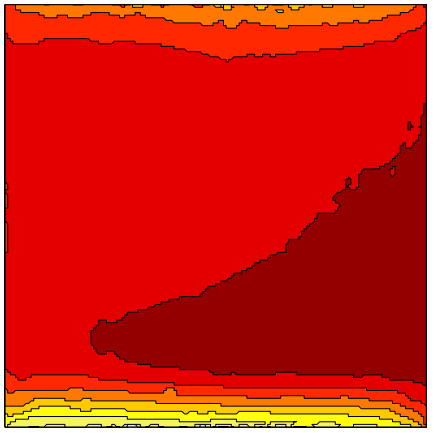

(c.2) $\mathrm{t}=90 \mathrm{~s}$

Figure 4-5: Temperature contours inside the cell during the initial isothermal stage One of the most important advantages of the MZI technique is to provide a transient view of the fluid field, which can be used for recalibration of the results or the measurement of experimental error in the system. Accordingly, even if there is a steady error in the measurement of temperature, it can be determined and removed from the final result. We analyzed the behavior of the measured refractive index variation in the field before application of the temperature gradient between cell sides. We expected to get close to zero average variation in the profile, as there was no source of heat to disturb the uniform distribution of refractive index in the system. In other words, the fluctuations in the profile during this period of time should represent the experimental errors in the system. However, in the next section we would like to prove that these fluctuations were not caused by the optical setup or by the post processing of the images. 
Figure 4-3 and Figure 4-4, respectively, illustrate the refractive index contours obtained from the front and side views of the IVIDIL cell for case 1. Parts a to $f$ in these figures show the refractive index field before application of the temperature gradient at six times, part $\mathrm{j}$ illustrates refractive index variation after $100 \mathrm{~s}$ of applying the temperature gradient, and part $\mathrm{k}$ shows this field at the end of the thermal time. While at first glance at $\Delta \mathrm{T}=0$, the contours seem to show random variation due to experimental error, a detailed look shows that each of these contours represents the trends in the system and was not caused by random errors in the optical set up. For instance, the curves in parts e, $\mathrm{f}$ and $\mathrm{g}$ are caused by a strong refractive index variation between the walls in the cells caused by the heating of the Peltier elements. There is always a gradient of the refractive index changes along the lateral walls ( $\mathrm{x}$ and $\mathrm{y}$ axis) caused by Peltier elements, but they are weaker signals in the rest of the contours.

In addition, comparing Figure 4-3 and Figure 4-4 shows that there were different 3D density fields inside the cell before applying the temperature gradient (particularly see Figure 4-3h and Figure 4-4b). This observation confirms our finding that these variations are not optical or post processing errors, due to the large magnitude in the variations. The refractive index variation before applying the temperature gradient $(\Delta \mathrm{T}=0 \mathrm{~K})$ is comparable with the refractive index variation after applying the temperature gradient. This means that there was a non-negligible variation of in the refractive index at $\Delta \mathrm{T}=0 \mathrm{~K}$. The thin gaps between the quartz cell and both the cold and hot walls of the cell cause a non-linearity of multiple contours that originates at the corners of the cavity [112].

Particularly, the internal side of the O-rings at the small gap between the copper and quartz is filled by the working liquid. On the other hand, the external side of the gap is filled by the surrounding gas that provides a large amount of heat transfer [65,66,109]. Thus, the largest thermal gradients arise at the corners of the cell, due to the high heat conductivity of the rubber seal and nearby regions (see Figure 4-3j,k and Figure 4-4j,k). Because we found three different kinds of temperature distributions before application of the temperature gradient, we selected three images from this time range $(t=25 \mathrm{~s}, \mathrm{t}=65 \mathrm{~s}$, and $\mathrm{t}=90 \mathrm{~s})$ that demonstrate these different conditions clearly. Figure 4-5a illustrates the first condition, which is a uniform relatively temperature (ideal case) in the system ( $298.15 \mathrm{~K})$. 


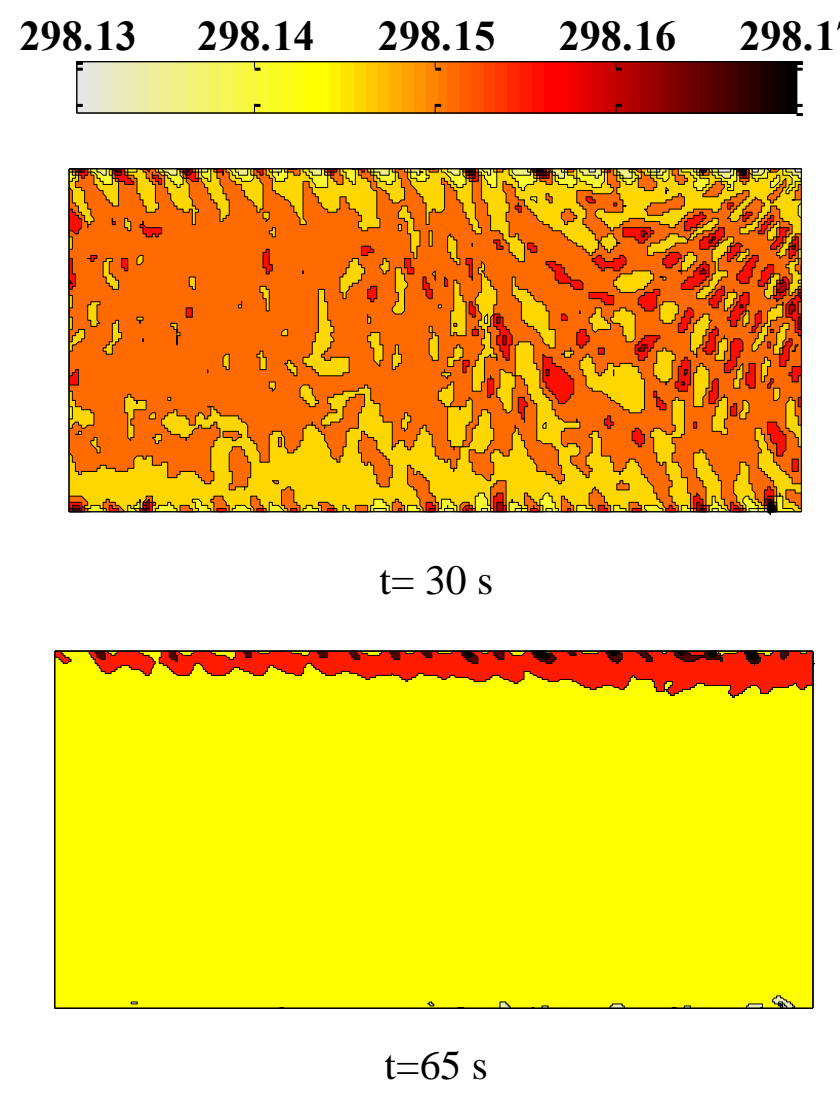

Figure 4-6: Temperature contours inside the cell during the initial isothermal stage for the case 2 with the $1[\mathrm{~cm}] \mathrm{x} 1[\mathrm{~cm}] \mathrm{x} 0.5[\mathrm{~cm}](\mathrm{x}, \mathrm{y}$, and $\mathrm{z})$ cell size

In this state, there is no heat flux coming from the copper blocks to the system, which reflects the condition in which the experiments should be run. The second condition occurred when noticeable temperature gradients close to walls were observed. In this condition, the cell was being cooled from one side and heated from the other side of the cell by the Peltier Elements to keep the temperature at $298.15 \mathrm{~K}$. In this condition, the maximum temperature difference close to walls was recorded $(0.25 \mathrm{~K})$. Here, we believe that the temperature controllers were overheating and/or overcooling the liquid using the Peltier elements. Because of this improper heating, the temperature of the system started deviating from the mean temperature until the changes could be detected by the thermal sensor. Thus, the temperature controller unit tries to return the liquid temperature to the mean temperature $(298.15 \mathrm{~K})$ by cooling or heating both sides. In this case (third condition), the maximum temperature deviation from the mean temperature in the domain can be seen at the center region of the cell and close to walls. This 
case represents the maximum temperature deviation from the ideal case (about $0.48 \mathrm{~K}$ as can be seen in Figure 4-5c).

\subsubsection{Thermal Analysis of the DSC Cell}

The results of the current case show that the thermal unit performance of the DSC facilities has been considerably improved. Temperature fields inside the cell 2 show a uniform temperature distribution before application of the temperature gradient to the system (see Figure 4-6). Figure 4-6a shows the very small fluctuations of the temperature $(<0.02 \mathrm{~K})$ inside cell 2 close to the walls, where the highest thermal deviations were expected; however, other images from the same time frame illustrate a quite smooth temperature distribution without any strong fluctuation (see Figure 4-6b). This demonstrates a good performance of the thermal controller unit of the DSC.

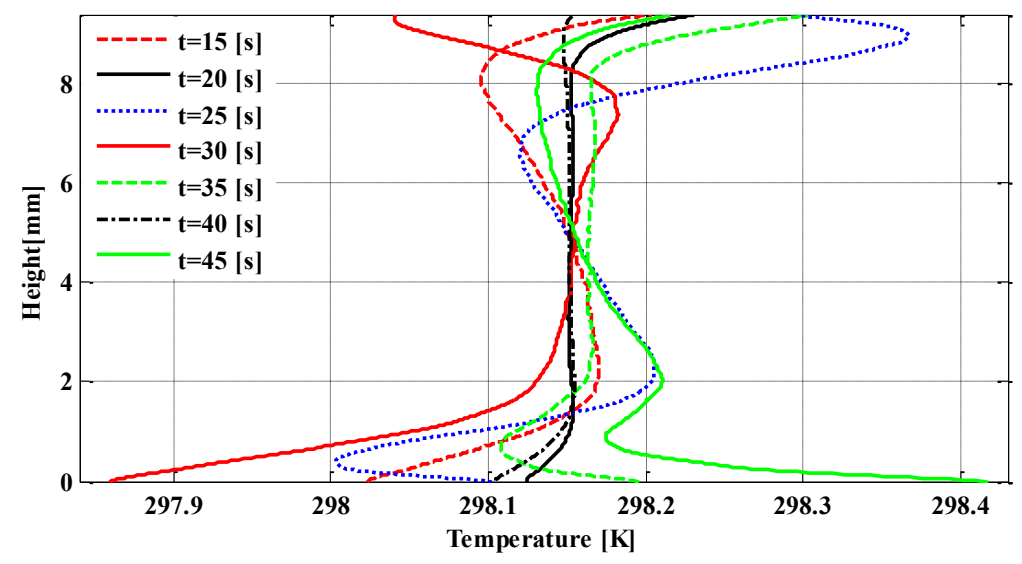

(a)Binay cell

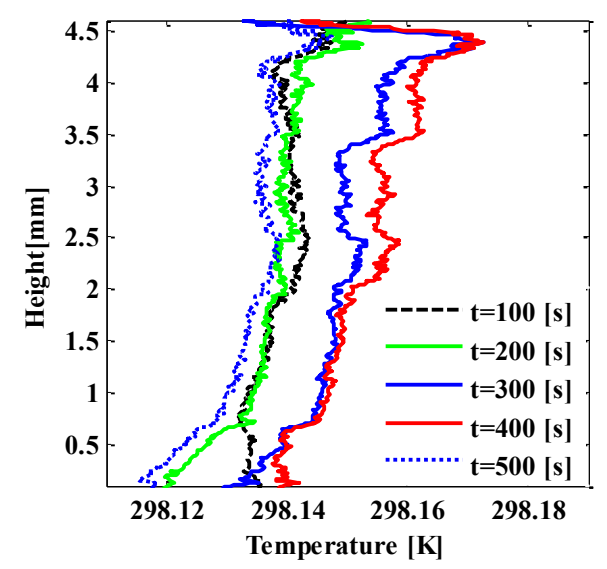

(b)Ternary cell

Figure 4-7: Temperature variation between the hot and cold walls

Temperature variations between the hot and cold walls at the center of the SODI cells at different times before the application of the temperature gradient are displayed in Figure 4-7. The temperature variation at the middle of cell 1 shows the existence of a large temperature gradient close to the walls; however, at the center of the cell $(z=2$ to $8 \mathrm{~mm})$, the variation of the temperature is kept below $0.05 \mathrm{~K}$. As mentioned earlier, the maximum variation from the mean temperature was observed at $t=30 \mathrm{~s}$. Figure $4-7 \mathrm{~b}$ shows the indicated temperature variation at the center of cell 2. The maximum fluctuation between both sides is less than $0.02 \mathrm{~K}$, and the 
fluctuation varies nearly linearly between both walls at different times. The sensitivity of the optical temperature measurement can be seen here as very small noise (order of $10^{-3} \mathrm{~K}$ ) along the plotted lines.
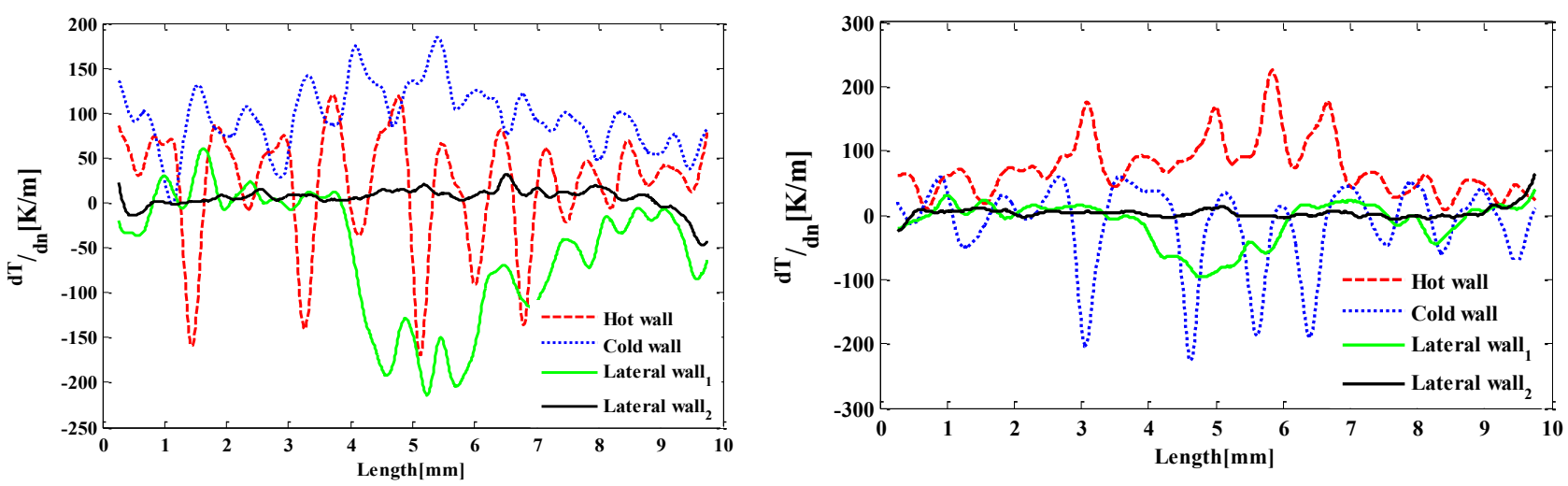

(a) before applying temperature gradient

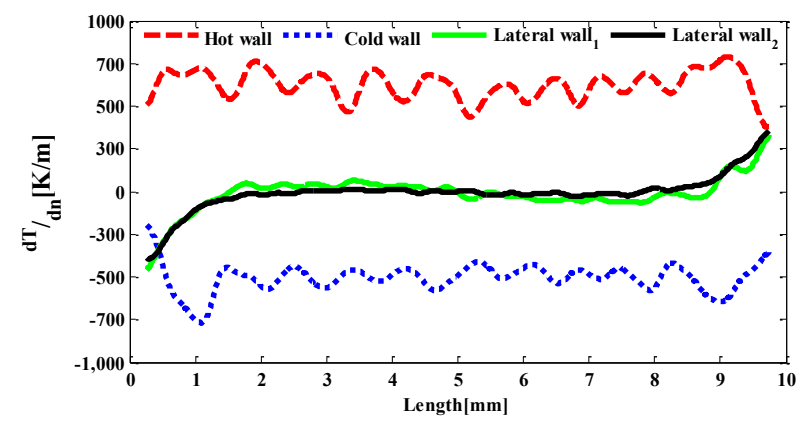

(c) during thermal time $\mathrm{t}=216 \mathrm{~s}$

(b) before applying temperature gradient

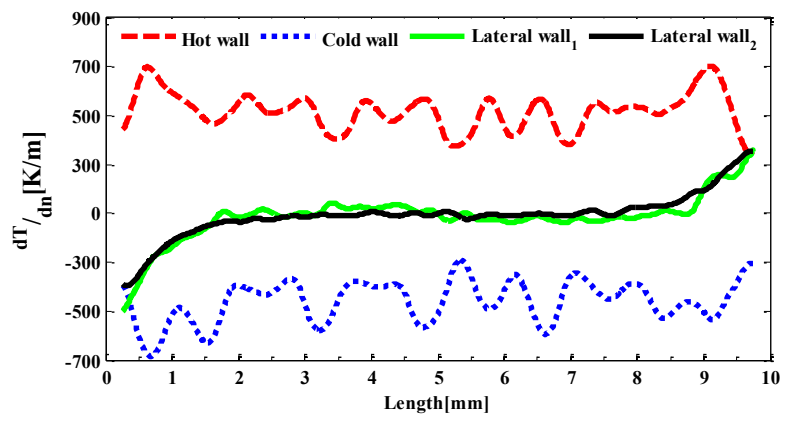

(d) at the end of thermal time $t=770 \mathrm{~s}$

Figure 4-8: Normal temperature gradient near boundaries of the case 1 with the $1[\mathrm{~cm}] \mathrm{x} 1[\mathrm{~cm}] \mathrm{x}[\mathrm{cm}]$ cell size

By comparing the performance of cell 2 with that of cell 1, we can determine that the temperature variation in case 1 was not due to optical or post-processing errors. It was found that the height of the cell contributed to the non-uniformity in the temperature profile. It must be noted that the only difference between the thermal set up of the IVIDIL and DSC facilities is the cell size. Therefore, we can conclude that using a cell with a smaller height in the present case (case 2; DSC experiment) and the combination of the thermal sensor and controller unit was more accurate than the case 1 with a greater distance between the thermal sensors. The smaller deviation of temperature from the targeted value at the central region of cell 1 supports this hypothesis (see Figure 4-7a). In addition, we believe that keeping both sides of the cell at same 
temperature is more sensitive task for the SODI thermal unit in comparison with keeping the specified thermal gradient between the cell walls for cell 1.

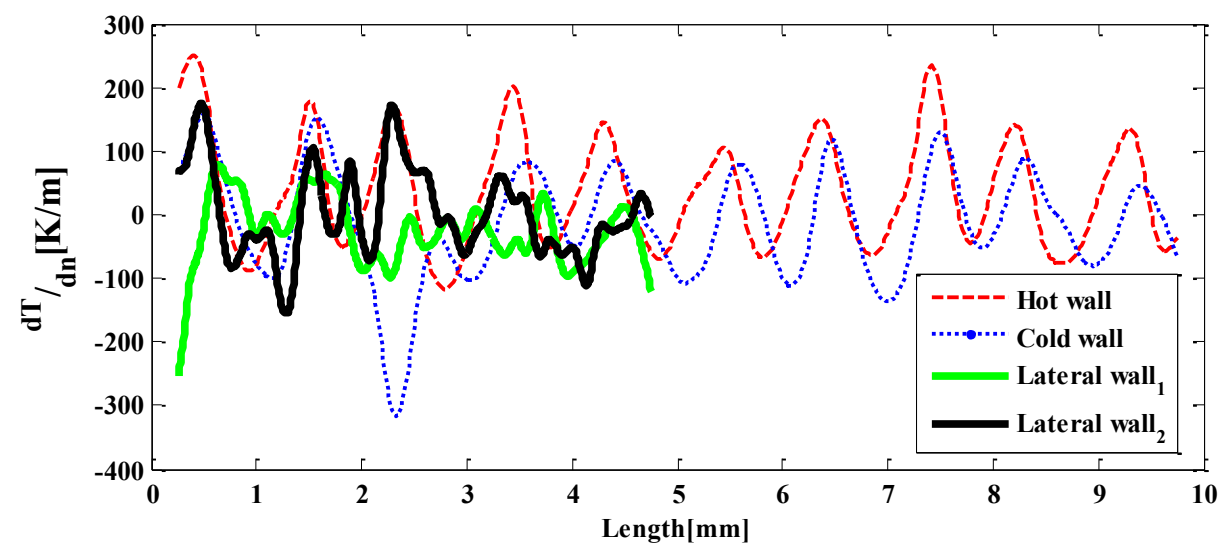

(a) before applying temperature gradient

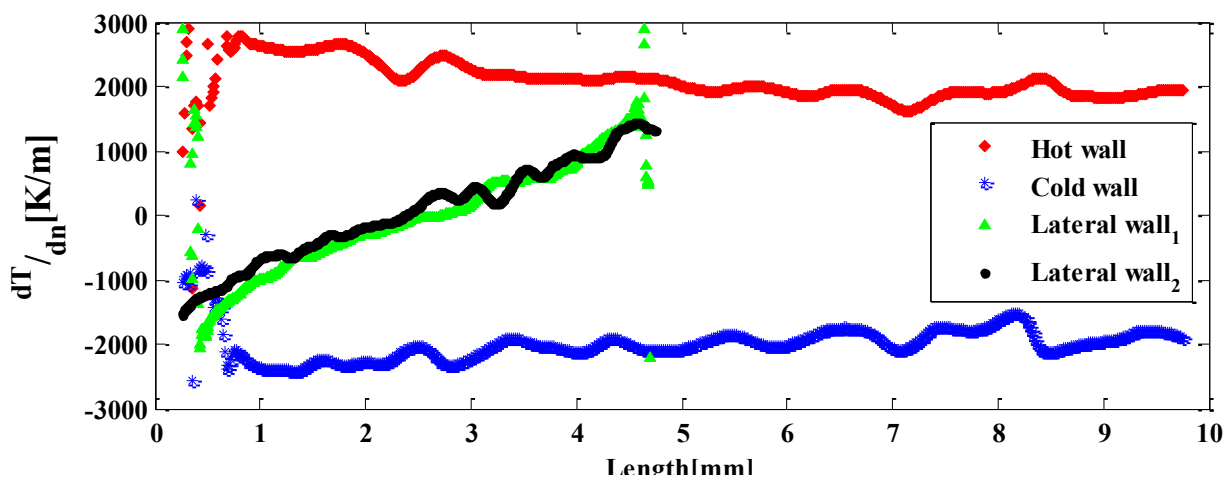

(b) at the end of thermal time $\mathrm{t}=330 \mathrm{~s}$

Figure 4-9: Normal temperature gradient near boundaries of the case 2 with the $1[\mathrm{~cm}] \mathrm{x} 1[\mathrm{~cm}] \mathrm{x} 0.5[\mathrm{~cm}](\mathrm{x}, \mathrm{y}$, and $\mathrm{z})$ cell size

As illustrated in Figure 4-1, the variations of the temperature close the lateral walls of the cell and along lines $\mathrm{L}_{2}$ and $\mathrm{L}_{3}$ are employed to study the heat transfer at the lateral walls. As shown in Figure 4-8a,b a noticeable, non-uniform temperature gradient existed prior to the heating of the cell. One can see a strong fluctuation the middle of the cavity and close to the lateral wall (the wall that is close to line $\left.\mathrm{L}_{2}\right)$ which may indicate a faulty insulation of the wall at that region $(\mathrm{z}=$ 3 to $6 \mathrm{~mm}$ ). At the other lateral wall (the wall 2 that is close to line $\mathrm{L}_{3}$ ), however, there is zero heat transfer at the middle range (except for a small variation near the hot and cold walls). Figure 4-8c,d show the normal temperature gradient during the heating process. The heat dissipation at the middle region of the lateral wall 1 was not easily observable after applying the 
temperature gradient, because the magnitude of the temperature gradient was higher during heating process. Figure $4-8 \mathrm{c}$ and $\mathrm{d}$ illustrate the temperature gradient in the middle of the cell during the first thermal time at $\mathrm{t}=216 \mathrm{~s}$ and right after the thermal time at $\mathrm{t}=770 \mathrm{~s}$. Noticeable heat dissipation at the lateral walls and close to the hot and cold walls were seen after application of the temperature gradient. When comparing the heat dissipation near the walls at two different times $(t=216 \mathrm{~s}$ and $t=770 \mathrm{~s})$, we notice that there is an increase in the heat dissipation with time at the corners until it reaches steady state at the end of the first thermal time. Consequently, after application of the temperature gradient, the most important source of unwanted heat transfer was that at the corners of the cells.

Figure 4-9 shows the average heat transfer along the laser path and close to the walls of cell 2 (DSC) at $\mathrm{t}=60 \mathrm{~s}$ (before heating the cell) and $\mathrm{t}=330 \mathrm{~s}$ (during the heating process). Similar fluctuations in the average heat transfer along all walls can be observed during the non-heating time (See Figure 4-9a). Although smooth fluctuations in the normal temperature were observed for case 2, there was large heat dissipation close to the lateral walls. These observations are in contrast to the findings from the square cavity of cell 1 . The magnitude of the heat dissipation at the lateral walls was about $2000 \mathrm{~K} / \mathrm{m}$. Thus, we believe that the linear heat dissipation from the hot to the cold walls of the cell 2 is indicative of poor thermal insulation in the system for this rectangular cavity as well. Having a higher assigned temperature difference between the heated walls in the case $2(\Delta \mathrm{T}=10 \mathrm{~K})$, and utilizing a cell with a smaller height have contributed to the larger heat dissipation for cell 2 at the lateral walls. Both of these factors should assist in increasing the absolute value of $\partial \mathrm{T} / \partial \mathrm{x}$ to $2000 \mathrm{~K} / \mathrm{m}(\Delta \mathrm{T} / \Delta \mathrm{x}=10 \mathrm{~K} / 5 \mathrm{~mm}=2000 \mathrm{~K} / \mathrm{m})$. These optically determined factors demonstrate the need to determine and remove the influence of this improper heat transfer from the measurement of the Soret effect. Accordingly, it must be noted that the region close to the lateral walls with the improper heat transfer and temperature variations should be removed from the calculation of the Soret coefficient and post processing must be repeated with resized and cropped images.

The difference between the lateral heat fluxes of the cells was elucidated by analyzing the unwrapped phase map when the temperature difference was applied, as shown in Figure 4-10. While parallel fringe patterns were detected inside cell 2 (except at the corners that the curvature 
of the fringes exist), we observed fully bended fringes close to the lateral sides of cell 2 . These bands should have been linear and parallel to the horizontal walls.

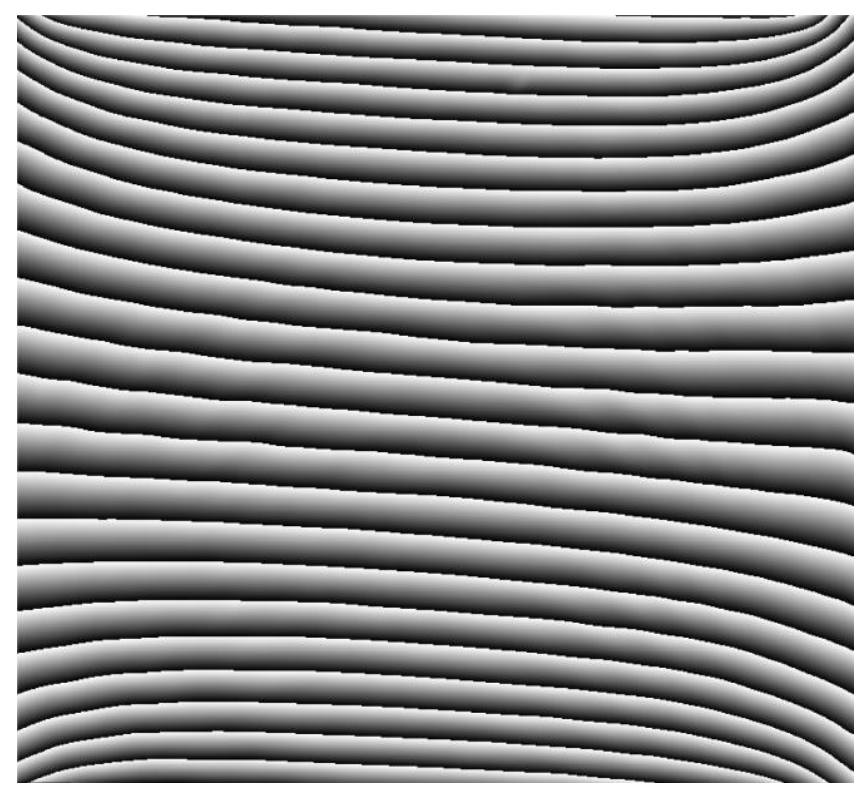

(a) case1 (IVIDIL-Run2R at $\mathrm{t}=700 \mathrm{~s}$ )

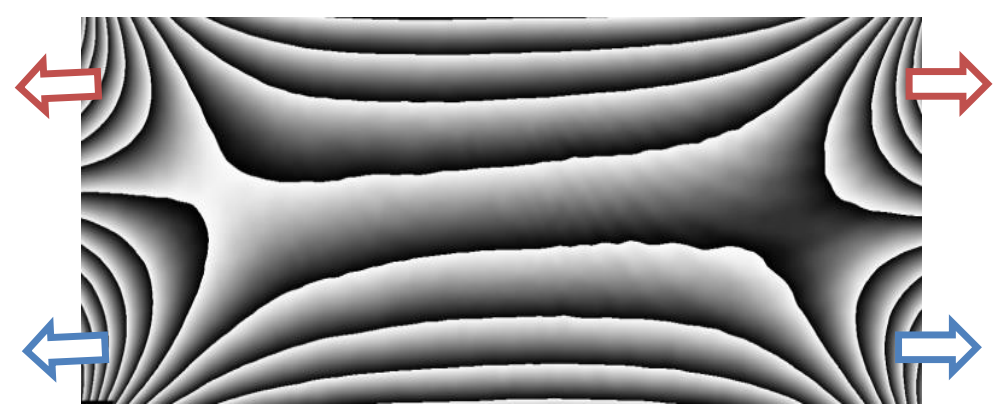

(b) case $2($ DSC-Run12 at $\mathrm{t}=130 \mathrm{~s})$

Figure 4-10: Wrapped phase distribution inside the SODI cells at the end of the thermal time

These curved bands can demonstrate the direction of the heat flux and reveal the pixels along the lateral walls where zero heat flux exists. Completely different patterns of heat transfer were observed at the bottom of the cavity close to the lateral walls, where the heat transfer moves from high to low from the quartz walls toward the middle of the cell. Moreover, these wrapped phases exhibit isolines of density that are directly affected by the temperature. As a result, the angles of these lines with respect to the wall surface indicate the strength of the heat flux. The zero-heatflux point (the only point along each lateral wall that shows a well-insulated condition) was close to the middle of the lateral walls where the isolines emerged perpendicular to the wall surface. It 
must be mentioned that these gradients were not strong enough to affect the entire cell; therefore, their effect on the separation process in the central region of the cell was minimal. As shown in the DSC phase map (Figure 4-10b), if the center region of cell ( $\mathrm{x} \sim 2.5$ to $7.5 \mathrm{~mm}$ ) along the $\mathrm{x}$ direction (see Figure 4-1) for all images was cropped and analyzed, the influence of lateral heat transfer from the system can be avoided. In this region, a purely thermo diffusive process can occur that should be used to measure the Soret coefficients of binary or ternary mixtures.

In order to assess the validity of these observations, we analyzed several more IVIDIL and DSC cases with a different assigned temperature difference $(\Delta \mathrm{T}=10 \mathrm{~K})$. The DSC cases always showed less than $0.02 \mathrm{~K}$ deviation from zero before application of the temperature gradient and then similar small fluctuations after application of the temperature gradient $\left(\Delta \mathrm{T}_{(\mathrm{t})}-\Delta \mathrm{T}_{\mathrm{st}}<0.02\right.$ $\mathrm{K})$. However for the cases of the IVIDIL cell (with a larger height), stronger fluctuations in the temperature gradient between the hot and cold sides were detected with different magnitudes when $\Delta \mathrm{T}=0$. We observed that the maximum temperature deviation differs for the various cases from $0.05 \mathrm{~K}$ to $0.40 \mathrm{~K}$. We also observed that although the IVIDIL temperature fluctuations were noticeable when the applied $\Delta \mathrm{T}$ was zero, in the presence of an applied temperature difference the thermal unit performed accurately. During the thermodiffusion phase, the temperature fluctuation was small enough be neglected. This shows the accuracy of the thermal part of the SODI apparatus after applying temperature gradient. It must be noted that even in this case, a smaller value of the steady temperature difference between the heated walls was found. This deviation must be considered in the Soret calculation.

The optical temperature measurement allows the sensitive performance of the controller unit before application of the temperature gradient; however, it also shows that the accuracy of the unit when $\Delta \mathrm{T}>0$ is good. It must be noted that in all cases the steady temperature difference between walls were less than the value assigned by the thermal controller unit. For example, the optically measured temperature difference was $4.45 \mathrm{~K}$ for case 1 with the applied $\Delta \mathrm{T}=5 \mathrm{~K}$, and for other cases with an applied $\Delta \mathrm{T}=10 \mathrm{~K}$ the measured temperature difference was between 9.15 and $9.30 \mathrm{~K}$ (Considering the entire height of the cells). We observed similar effects for the DSC cases with an applied $\Delta \mathrm{T}=10 \mathrm{~K}$. We believe that although the thermal conductivity of the copper blocks is high, the distance between the thermal sensors and the liquid surface causes the actual temperature difference sensed by the liquid at the inner sides of the cell to be less than the 
applied values. Furthermore, while the thermal conductivity of the copper was analyzed numerically inside the cell array to apply the assigned $\Delta \mathrm{T}[114]$, this behavior was not predicted. It is worthwhile to remark that the optically obtained temperature difference is for the cropped span. Nevertheless, distance that is lost due to cropping is small enough $(\sim 0.4 \mathrm{~mm})$ that it should not cause this magnitude of observed temperature difference.

Table 9: Summary of the thermal analysis results of the both IVIDIL and DSC cases

\begin{tabular}{|c|c|c|c|c|c|c|c|c|c|c|c|c|}
\hline \multirow{2}{*}{$\begin{array}{l}\text { 尺ी } \\
\text { है }\end{array}$} & \multirow{2}{*}{$\begin{array}{c}\text { Cell } \\
\text { size } \\
{\left[\mathrm{cm}^{3}\right]}\end{array}$} & \multirow[t]{2}{*}{$\begin{array}{l}\text { Peltier } \\
\Delta \mathrm{T}[\mathrm{K}]\end{array}$} & \multirow[t]{2}{*}{$\begin{array}{l}\text { Optical } \\
\Delta \mathrm{T}[\mathrm{K}]\end{array}$} & \multicolumn{8}{|c|}{$\begin{array}{l}\text { Average temperature gradient normal to the sides of the cell } \\
\qquad \pm 5[\mathrm{~K} / \mathrm{m}]\end{array}$} & \multirow[t]{2}{*}{$\begin{array}{c}\Delta C \\
{[-] / 10^{-3}}\end{array}$} \\
\hline & & & & $\begin{array}{l}\text { Hot } \\
\text { wall }\end{array}$ & $\begin{array}{l}\text { Cold } \\
\text { wall }\end{array}$ & $\begin{array}{c}\text { Lateral } \\
\text { wall }_{1}\end{array}$ & $\begin{array}{c}\text { Lateral } \\
\text { wall }_{2}\end{array}$ & $\begin{array}{r}\text { Half } \\
\text { nes }\end{array}$ & $\begin{array}{l}\text { valls } \\
\text { hot }\end{array}$ & $\begin{array}{r}\mathrm{Hal} \\
\mathrm{ar}\end{array}$ & $\begin{array}{l}\text { walls } \\
\text { old }\end{array}$ & \\
\hline 1 & $1 \times 1 \times 1$ & 0 & 0.30 & 98 & 131 & 51 & 11 & 41 & 10 & 63 & 15 & 0 \\
\hline 2 & $1 \times 0.5 \times 1$ & 0 & 0.02 & 35 & 32 & 5 & 8 & 4 & 4 & 5 & 4 & 0 \\
\hline 1 & $1 \times 1 \times 1$ & 5 & 4.23 & 687 & 643 & 18 & 29 & 101 & 89 & 94 & 87 & 3.17 \\
\hline 2 & $1 \times 0.5 \times 1$ & 10 & 8.37 & 2246 & 2020 & 20 & 34 & 540 & 567 & 520 & 578 & 6.20 \\
\hline
\end{tabular}

The summary of the thermal results of the IVIDIL cell(case 1) and the DSC cell (case 2) performed on board the ISS are reported in Table 9, both before application of the temperature gradient and at the end of the thermal time. At $\Delta \mathrm{T}=0$, zero heat flux must be obtained from all walls; however, as a result of lateral heat transfer a non-zero mean value close to the walls was seen for cell 1.

Heat leakage from the lateral walls, in general, was greater for cell 2 due to its smaller height (compared to cell 1). Interestingly, the average temperature gradient value close to one lateral wall of the IVIDIL cell was five times larger than the other lateral walls when the applied $\Delta \mathrm{T}=$ 0 . This could be caused by weaker insulation performance of this wall. The maximum temperature difference between the walls was measured as about 11 percent less than the applied $\Delta \mathrm{T}$ by the thermal controller unit. According to theory, in the presence of a thermal difference between walls, $\partial \mathrm{T} / \partial \mathrm{y}$ close to both the hot and cold walls should be $500 \mathrm{~K} / \mathrm{m}$ for case 1 and 2000 $\mathrm{K} / \mathrm{m}$ for case 2 . The observed values, however, are higher than the theoretical values. The main reason behind this deviation from theory is the faulty insulation at the lateral walls, which has a more pronounced effect at the corners of the cell. All of these findings were obtained optically and not by the thermocouple sensors. It must be noted that the measured temperature difference is less than the applied value, but the average temperature is greater. This behavior is because of the high heat transfer at the corners of the cell that affected only the average value. The detailed 
contour information that produced this interesting conclusion would not have been obtained only using the thermocouples. This shows the essential importance of using optical results to measure the temperature variation in the cell.

The lateral heat flux close to the thinner cell was more pronounced than that of the thicker cell, as the absolute value of $\partial \mathrm{T} / \partial \mathrm{x}$ had the same order of magnitude $(\sim 1100 \mathrm{~K} / \mathrm{m})$ as $\partial \mathrm{T} / \partial \mathrm{y}$ close to the hot or cold walls $(\sim 2100 \mathrm{~K} / \mathrm{m})$ of cell 2 . As the height of cell 2 is half that of cell 1 , and the assigned $\Delta \mathrm{T}$ is two times greater, the lateral heat fluxes in cell 2 were four times greater than those in cell 1. Owing to the capability of the MZI experiment, all of these parameters can be found and considered during the post processing, which is a valuable advantage of the optical measurement technique.

While the thermal controller unit of SODI provided accurate performance during the thermodiffusion step of the IVIDIL and DSC experiments when $\Delta \mathrm{T}>0$, it is essential to utilize optical methods to extract the accurate thermal results during the experiment, because they provide the valuable and detailed information of the thermal performance of the system. These results can be employed to adjust the processing method or recalibrate the system accordingly.

Table 10: Comparison of the Soret coefficient calculated using $\Delta \mathrm{T}$ measured optically and using thermal sensor

\begin{tabular}{|c|c|c|c|c|c|c|}
\hline & $\begin{array}{c}\text { Ref } \\
{[114]}\end{array}$ & Ref [109] & $\begin{array}{c}\text { Ref } \\
{[75,85]}\end{array}$ & $\begin{array}{c}\text { Benchmark } \\
\text { Value[75] }\end{array}$ & $\begin{array}{c}\text { Our work using } \Delta \mathrm{T} \\
\text { of Peltier Elements }\end{array}$ & $\begin{array}{c}\text { Our work using } \Delta \mathrm{T} \text { of } \\
\text { Optical Results }\end{array}$ \\
\hline $\mathrm{S}_{\mathrm{T}} / 10^{-3}\left[\mathrm{~K}^{-1}\right]$ & 7.08 & $6.77 \pm 0.11$ & 8.87 & $8.47 \pm 1.5$ & $7.16 \pm 0.2$ & $8.56 \pm 0.2$ \\
\hline
\end{tabular}

Other studies have used the thermal sensors data to measure the Soret coefficient of a water/IPA mixture with the initial mass fraction of $10 \%$ of IPA $[66,109]$. The $S_{T}$ values determined from those studies are reported in Table 10. However, the IVIDIL results using the thermal sensor elements underestimates the Soret coefficient compared to the results obtained when using the optical beam deflection of Koehler et al. [85] or the benchmark value of $S_{\mathrm{T}}$ for this mixture [75]. The measured $S_{T}$ from the optical temperature results provides a better match with the benchmark value and optical beam deflection results with less than $3 \%$ deviation. This shows the accuracy and necessity of using and considering the optically measured temperature in the post processing of MZI experimental images. This method can be used to improve the boundary conditions of numerical simulations by imposing a realistic heat loss on the lateral walls, rather than assuming a perfectly insulated boundary. 


\subsection{Conclusions}

We observed that the sensitivity of the temperature measurement using MZI for the SODI facilities is on the order of $10^{-3} \mathrm{~K}$ according to the current image processing techniques. This means that stronger fluctuations in the temperature in the profile were not caused by optical or post processing errors. Despite the relatively weaker performance of the IVIDIL thermal controller unit at $\Delta \mathrm{T}=0$, temperature fields inside cell 2 showed a uniform temperature field before the application of the temperature gradient consisting of temperature variations of less than $0.02 \mathrm{~K}$. The optical temperature measurement proved the sensitivity of the controller unit before applying the temperature gradient; however, it also showed the accuracy of unit when $\Delta \mathrm{T}$ $>0$. When the applied $\Delta \mathrm{T}=0$, the temperature variation at the middle of cell 1 causes a high temperature gradient close to the walls; however, at the central region of the cell, the variation of the temperature was less than $0.05 \mathrm{~K}$. For cell 2 (in the DSC experiment), the combination of the thermal sensor and controller unit was more accurate than in case 1 for the measurement with the larger distance between the thermal sensors. The heat flux close to the lateral walls of cell 2 was stronger than that in case 1 , because not only the insulation was not sufficient, but also a linear variation of heat fluxes from the cold wall to the hot wall was seen. Based on the capability of the MZI thermodiffusion experiment, temperature deviations from the ideal case can be measured and its effects may be removed during the post processing of the Soret experiment results. This advantage cannot easily be gained using other experimental methods. Thus, the MZI technique can provide an accurate transient view of the field that can be used to adjust the postprocessing procedure in a way to measure a precise value for the maximum temperature difference and the Soret coefficient.

In all cases, the steady temperature difference between the hot and cold sides, measured optically, differed between $6 \%$ and $11 \%$ from the value reported by the thermal controller unit. Moreover, it was demonstrated that the fringe pattern close to the lateral walls of cell 2 should be cropped before processing the results. The measured $S_{T}$ using the optical temperature results provides a better agreement with the benchmark value and the previously known optical beam deflection results within a $3 \%$ deviation; however, using the thermal sensor temperature difference provided about a $10 \%$ deviation from the benchmark value. The results of this study 
can also be used for numerical models of thermodiffusion experiments in which the boundary condition can be accurately simulated. Furthermore, we showed that it is not an accurate assumption to consider zero heat flux on the lateral walls of either of the cells. 


\section{Chapter 5: Heat and Mass Transport Phenomena under the Influence of Vibration Using a New Aided Image Processing Approach}

\subsection{Introduction}

Microgravity suggests conditions that remove convection that reduces the separation of components in a mixture. Thus, the IVIDI experiment was proposed to study the impact of force vibrations on the mass transfer in the system due to Soret effect. The numerical analysis of Srinivasan et al. [62] showed that forcing the type of vibrations seen on the ISS had a significant effect on the Soret phenomena. They observed the existence of a single convective flow cell. They claimed that such a convection cell, can remove the influence of Soret separation [62]. It must be noted that the level of g-jitter vibration that applied during each individual experiment was the most important parameter of that experiment. For example, when the ISS microvibration condition was normal $\left(\operatorname{Vib}_{\mathrm{RMS}}<10^{-5}\right)$, pure thermodiffusion occurred and as a result an accurate $S_{\mathrm{T}}$ close to the benchmark value was found. The $\mathrm{g}$-jitter vibrations during all runs were considered in this study, and, excluding a few short periods (less than $10 \mathrm{~s}$ ), the ISS provided a suitable condition for thermodiffusion experiments. It is worth noting that Gershuni and Zhukhovitskii performed a series of studies on the hydrodynamics and stability of fluid flow subjected to an oscillatory gravity field [44,127-130].

\subsection{Chapter Aims}

In this chapter, the influences of a wide range of Rayleigh vibrations on the measurement of thermodiffusion in a microgravity environment subjected to a constant temperature difference between two walls of cubic cavity are presented for the first time in detail. Particularly, the effects of different parameters of vibrational forces, such as frequency and amplitude, on thermodiffusion experiments are studied. The impact on the separation of the components of the mixture due to the change in the forced vibration from low to high Rayleigh vibration are investigated. Nine different runs of a water and isopropanol mixture with a negative Soret coefficient and the same temperature difference were chosen as test cases in this study. In this 
work, the collected data are analyzed by the use of fast Fourier transform (FFT) image processing. A curve-fitting method based on a genetic algorithm, which is aided by a fast robust version of discretized smoothing, is then used to calculate the Soret coefficient. Results show maximum separation and Soret coefficient for the case with minimum Rayleigh number; however, a linear relation between the Rayleigh vibration and the maximum separation was not detected.
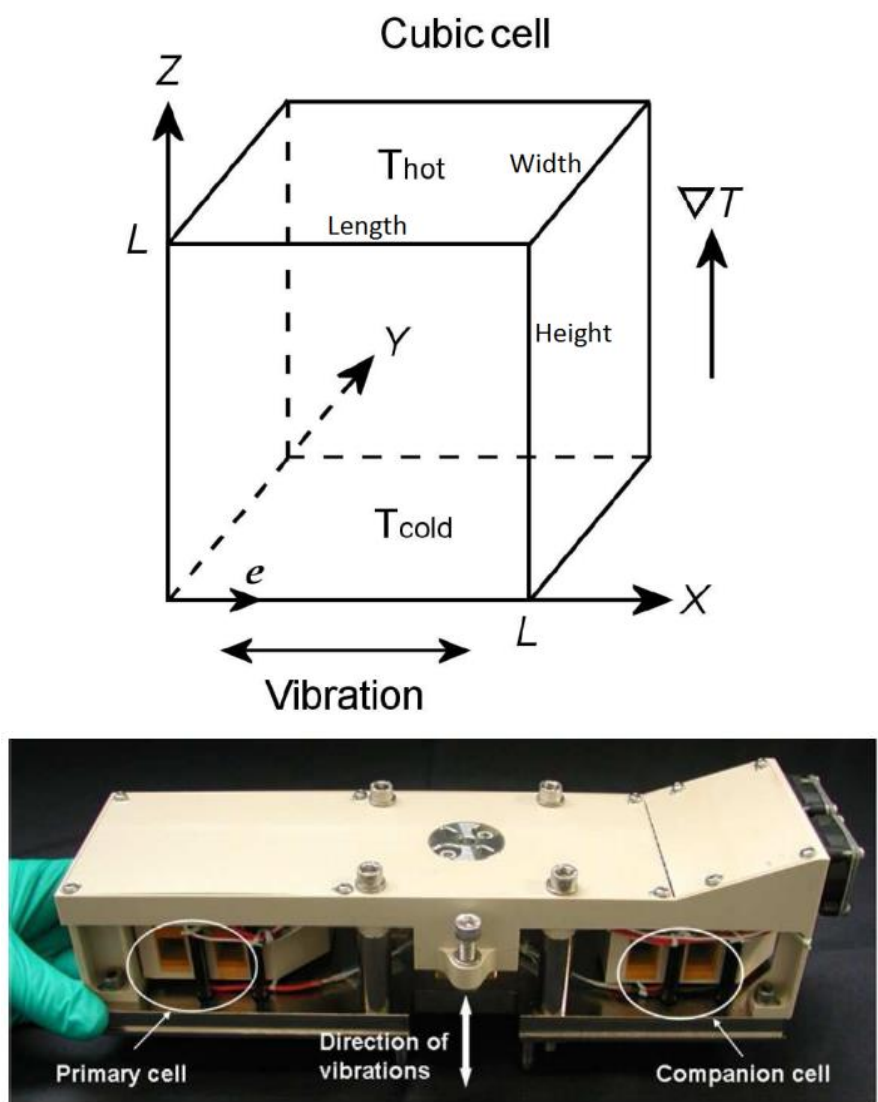

Figure 5-1: IVIDIL cell array and cell conditions [131]

The experimental mixture selected was water $(90 \% \mathrm{wt})$ and isopropanol $\left(\mathrm{C}_{3} \mathrm{H}_{7} \mathrm{OH}\right)$ subjected to a temperature gradient normal to the vibrational force. Figure 5-1 shows the directions of the temperature difference and of the control vibration applied to the cell array. Accordingly, the maximum concentration difference in the cell caused by Soret effect would be obtained from this process. Then the Soret coefficient is measured first by assuming the system has reached steady state and then using a genetic algorithm (GA) and curve-fitting procedure. 


\subsection{Experimental Setup}

SODI (Selectable Optical Diagnostics Instrument) was developed as a multi-purpose facility to perform various experiments such as IVIDIL, Diffusion Coefficients in Mixtures (DCMIX), and DSC. The first series of SODI experiments was IVIDIL and started on the $5^{\text {th }}$ of October 2009. Each run lasted 18 hours and was controlled via telescience. Each experimental run was conducted in two steps, one of 12 hours in the presence of a thermal gradient (thermodiffusion phase) and one of 6 hours at the mean temperature (diffusion phase). The forced vibrations with different amplitudes and frequencies were applied during these 18 hours. The level of on board g-jitter was recorded by SAMS (Space Acceleration Measurement System).

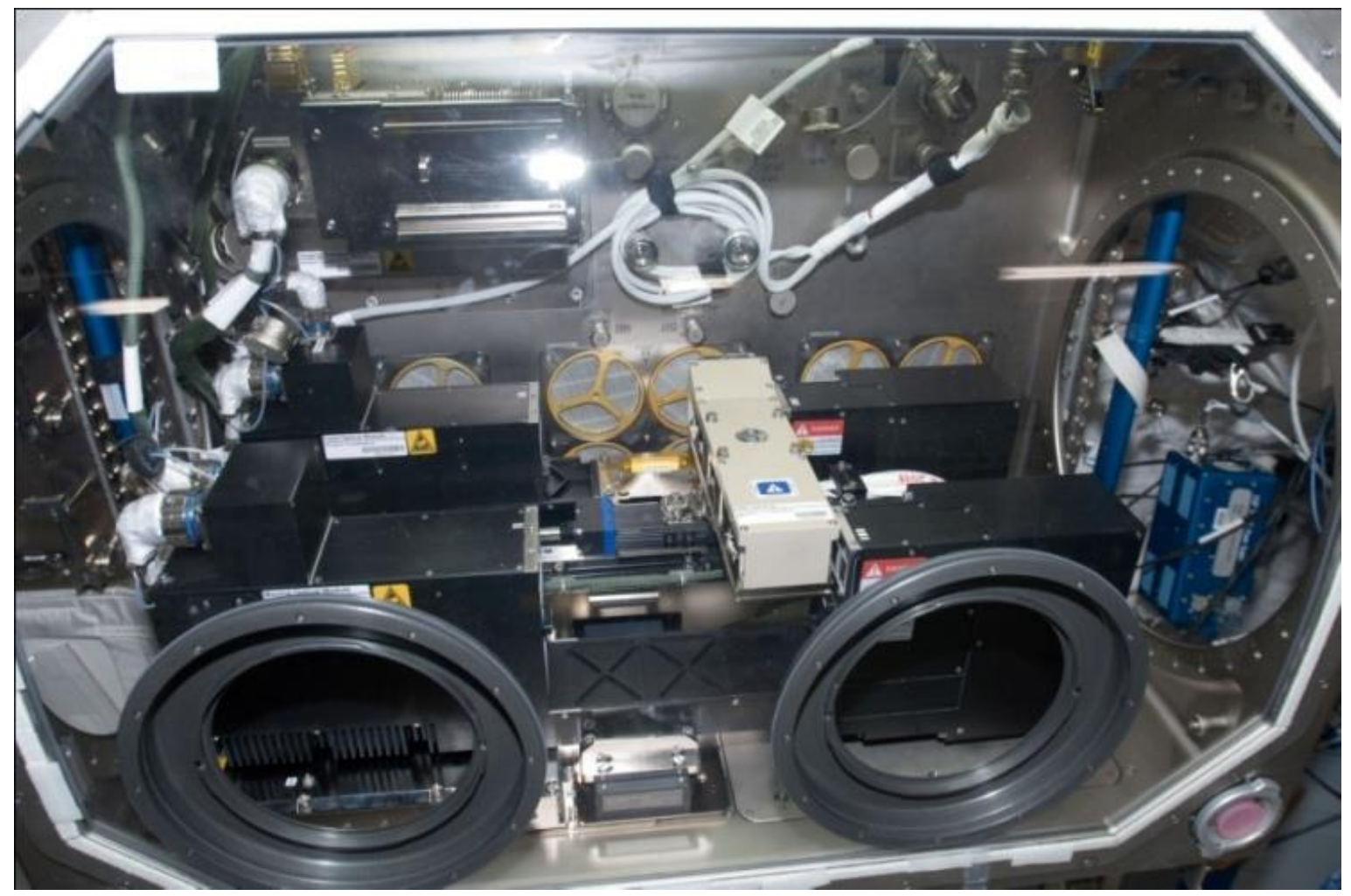

Figure 5-2: Thermodiffusion experimental setup [132]

Figure 5-2 shows the implemented version of the interferometer in combination with equipment for digitally recording the phase information and the experimental cell array that is installed inside of the glove box. The cell configuration was chosen according to optimization analysis of various aspect ratios of Soret cells [114]. Because of the vibrations and temperature gradient across the cell, the thermogravitational flow patterns in the liquid appear. 
These vibrational flows are characterized by the vibrational analogue of the Rayleigh number (Rayleigh vibration or Gershuni number) [127].

$$
R a_{v i b}=G s=\frac{\left(A \omega \beta_{T} \Delta T L\right)^{2}}{2 v \chi}
$$

where $\omega=2 \pi \mathrm{f}$ represents the angular frequency, A denotes the amplitude of the vibrations, $\mathrm{L}$ measures the characteristic size of the vibration, $\Delta \mathrm{T}$ is the applied temperature gradient, $v$ is the kinematic viscosity, $\beta_{\mathrm{T}}$ is the thermal expansion coefficient, and $\chi$ is the thermal diffusivity[127].

\subsection{Experimental Sequences}

The cavity was filled with a water and isopropanol (IPA) binary mixture with an identical composition for all cases, which provides a negative Soret coefficient. The first step was to initialize mixture characteristics by reaching a uniform concentration and temperature inside the cavity. For the first installation, to detach tracers that were stuck to the cell walls and ensure a homogeneous mixture, the cell array was shaken. After that, the cell and interferometer were stabilized at the desired mean temperature; this process lasts approximately 4 to 6 hours. After the mean temperature was established at $25^{\circ} \mathrm{C}$, a thermal gradient was applied across the cell.

A relaxation factor dictates the time required for the diffusion processes, such as the thermal time at the beginning of the set up. The thermodiffusion phase lasted $12 \mathrm{~h}$ during the IVIDIL experiments for the water/IPA mixtures, thus the diffusion time was assumed to be about $12 \mathrm{~h}$ for this experiment, which is smaller than the mentioned relation.

This is the time after which the separation of species is expected to become stabilized in the mixture. On the other hand, it is clear that $12 \mathrm{~h}$ is not enough to reach steady state for separation of this mixture, considering there was a noticeable amount of separation during the last hours of experiment on board the ISS, as well as the maximum separation for Run Ext1 which did not occur until after $22 \mathrm{~h}$ of the experiment had passed (c.f Figure 5-3). 


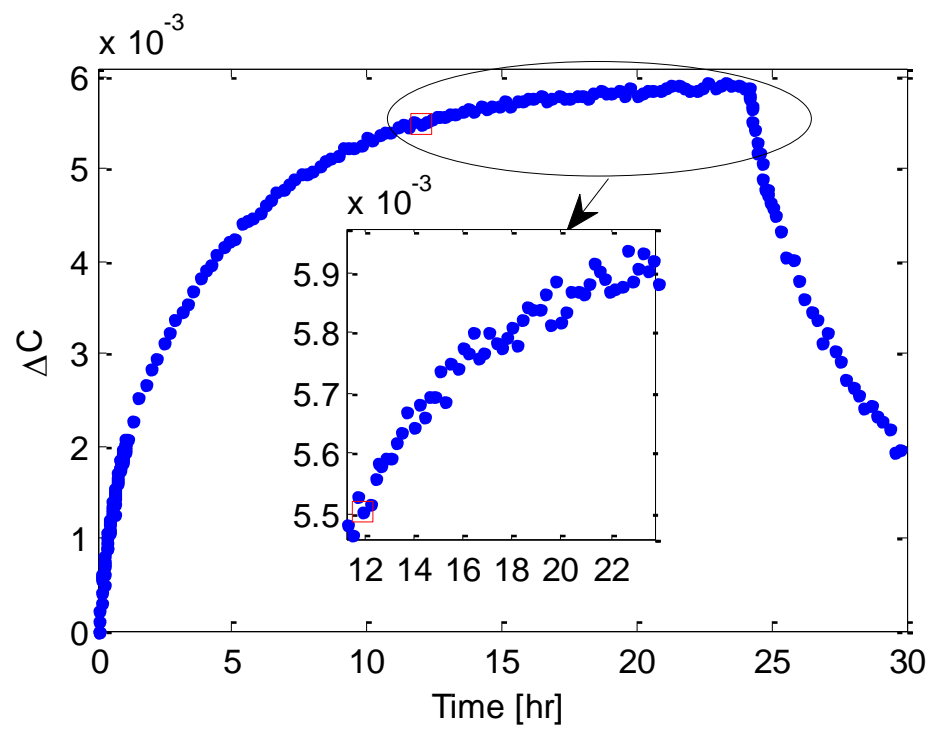

Figure 5-3: Separation versus time during entire thermodiffusion and diffusion phases of RunExt1

Consequently, in this study, a GA with fast robust discretized smoothing was implemented to find the best match curve with the experimental separation. GA results accurately estimate the Soret and diffusion coefficients simultaneously with a particular objective function.

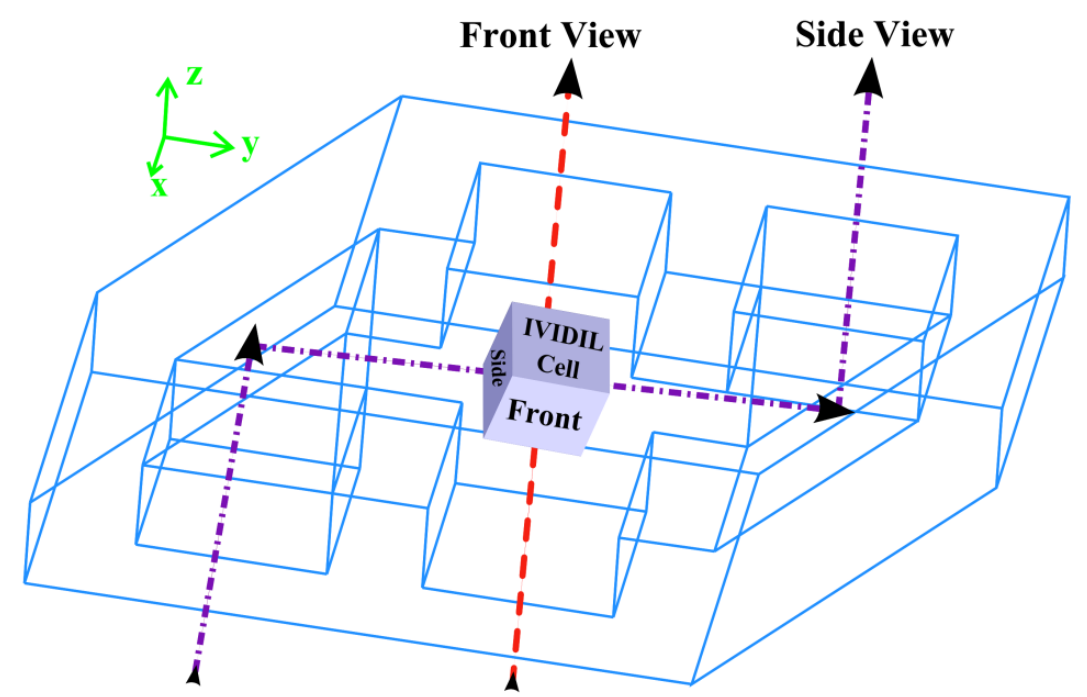

Figure 5-4: Sketch of two leaser paths inside the IVIDIL cell array

In this chapter, nine experimental runs (Run5 to Run12, inclusive, and RunExt1) according to Table 11 are investigated in detail. These experimental runs have similar $\Delta \mathrm{T}=10 \mathrm{~K}$, but vary 
from a low Rayleigh vibration of 1.3, which corresponds to Run12, to a high Rayleigh vibration value of 1392.1, which was in to Run11.

Table 11: Physical properties of the water-IPA mixture measured at T=298K $[65,109,132]$

\begin{tabular}{|c|c|c|c|c|c|c|c|c|c|}
\hline $\begin{array}{c}c_{o, I P A} \\
{[\mathrm{wt}-]}\end{array}$ & $\begin{array}{c}\mu \\
{[\mathrm{mPa} \cdot \mathrm{s}]}\end{array}$ & $\begin{array}{c}\beta_{T} / 10^{-4} \\
{\left[\mathrm{~K}^{-1}\right]}\end{array}$ & $\begin{array}{r}\beta_{C} / 10^{-1} \\
{[-]}\end{array}$ & $\begin{array}{r}\rho / 10^{2} \\
{\left[\mathrm{~kg} \cdot \mathrm{m}^{-3}\right]}\end{array}$ & $\begin{array}{r}(\partial n / \partial T)_{p, c} \\
/ 10^{-4}\left[\mathrm{~K}^{-1}\right]\end{array}$ & $\begin{array}{r}(\partial n / \partial c)_{p, T} \\
/ 10^{-2}[-]\end{array}$ & $\begin{array}{c}S_{T} / 10^{-3} \\
{\left[K^{-1}\right]}\end{array}$ & $\begin{array}{c}D / 10^{-10} \\
{\left[\mathrm{~m}^{2} \mathrm{~s}^{-1}\right]}\end{array}$ & $\begin{array}{c}D_{T} / 10^{-13} \\
{\left[\mathrm{~m}^{2} \mathrm{~s}^{-1} \mathrm{~K}^{-1}\right]}\end{array}$ \\
\hline $10 \%$ & 1.415 & 3.334 & 1.437 & 9.809 & 1.3427 & -9.2305 & $-8.47 \pm 1.5$ & $7.11 \pm 0.3$ & $-6.01 \pm 0.2$ \\
\hline
\end{tabular}

All of the above-mentioned procedures were used to estimate $S_{T}$; however, in this work forced vibration disturbed the pure diffusion pattern in the cavity. Moreover, $S_{\mathrm{T}}$ is one of the properties that should be measured in the convection less regime. In the bounds of this study, the measurements of the Soret coefficients are used to compare the strength of separation and the influence of the vibration during each run. In other words, this parameter is only used to have a precise comparison between runs that show similar characteristics during the experiment.

\subsection{Results and Discussion}

In this study, several parameters such as temperature and concentration fields in the cavity were studied. Digital optical interferometry provided a view over the entire cell and therefore allowed identification of the convection-free zones, temperature distribution, and concentration distribution in the cell at any location. In this study, $\Delta \mathrm{T}$ was kept at constant value of $10 \mathrm{~K}$ for all nine runs. Conversely, the frequency and amplitude of the applied vibration vary between each run. The main objective is to not only investigate any possible effect of vibration on the measurement of the Soret coefficient, but also to evaluate the sensitivity of the measurements to those effects. Thus, the behavior of a water-isopropanol mixture was studied under various Rayleigh vibration levels. The results were then compared with the analytical curve generated in the convection-free regime without any force or g-jitter vibration using benchmark values of the diffusion coefficients.

\subsubsection{Thermal Time and Temperature Profile}

A linear variation of the temperature is reached in the cavity at the end of thermal time. According to the given relation for thermal time stated in section 3, this parameter for a water/IPA mixture is close to $770 \mathrm{~s}$. Consequently, changes in the refractive index in this phase 
occur due to temperature changes in the cell. Liquid properties and contrast factors at the mean temperature of $298 \mathrm{~K}$, which can be found in Table 12, are used to determine the temperature field in the cell at each pixel of image, with error less than $0.1 \mathrm{~K}$.

Table 12: The IVIDIL experimental runs with 10K temperature gradient onboard ISS

\begin{tabular}{|c|c|c|c|c|}
\hline Run\# & $\mathrm{C}_{0 \text {-IPA }}$ & $\mathrm{F}[\mathrm{Hz}]$ & $\mathrm{A}[\mathrm{mm}]$ & $\mathrm{Ra}_{\text {vib }}$ \\
\hline Run 5 & $10 \%$ & 2.0 & 44 & 801.4 \\
\hline Run 6 & $10 \%$ & 2.8 & 25 & 507.1 \\
\hline Run 7 & $10 \%$ & 2.0 & 33 & 450.8 \\
\hline Run 8 & $10 \%$ & 1.0 & 70 & 507.1 \\
\hline Run 9 & $10 \%$ & 0.5 & 70 & 126.8 \\
\hline Run 10 & $10 \%$ & 0.2 & 70 & 20.3 \\
\hline Run 11 & $10 \%$ & 2.0 & 58 & 1392.5 \\
\hline Run 12 & $10 \%$ & 0.05 & 70 & 1.3 \\
\hline Run Ext1 & $10 \%$ & 1.0 & 70 & 507.1 \\
\hline
\end{tabular}

The separation of components began as soon as the thermal gradient was applied, and because separation is a slow process, neglecting a few minutes may not cause a noticeable influence. Figure 5-5 shows the maximum temperature difference from $100 \mathrm{~s}$ before application of the temperature gradient until the end of thermal time. The transient temperature phase is approximately finished after three minutes of applying the thermal gradient. Subsequently, the temperature behaves similar to the period that a linear temperature variation is established between the two walls, which occurs after thermal time.

When measuring the temperature field in the cavity experimentally, some interesting points were noticed. First of all, the maximum and minimum temperature variations between the two hot and cold walls were $8.65 \mathrm{~K}$ and $7.85 \mathrm{~K}$ in Run10 and Run11, respectively. This variation shows that a considerable change in the temperature variation among different runs that may affect the maximum separation. The reason is the direct effect of the temperature difference on the separation of the components. Second fact that may be extracted from Figure 5-5 is the maximum temperature difference value. It is supposed to be $10 \mathrm{~K}$ for this set of experiments; whereas, less than 9Kof temperature difference is detected for all runs. Consequently, substantial deviation of ideal thermodiffusion can be resulted by considering separations at the end of these runs due to $10 \mathrm{~K}$ temperature difference. Therefore, this fact shows the importance of measuring temperature in the cavity experimentally. 
The variation of temperature during first $100 \mathrm{~s}$ of experiment is less than $0.1 \mathrm{~K}$ for all runs that corresponds to isothermal condition. This small deviation of temperature shows a reliable operating condition of IVIDIL runs, which was caused by the thermal unit. The negative sign of the temperature difference in Figure 5-5 means the assigned hot wall was cooling the cell at that time. More studies are required to define an accurate thermal time for diffusion experiments. The reason is that even a small separation during this period may have small effects.

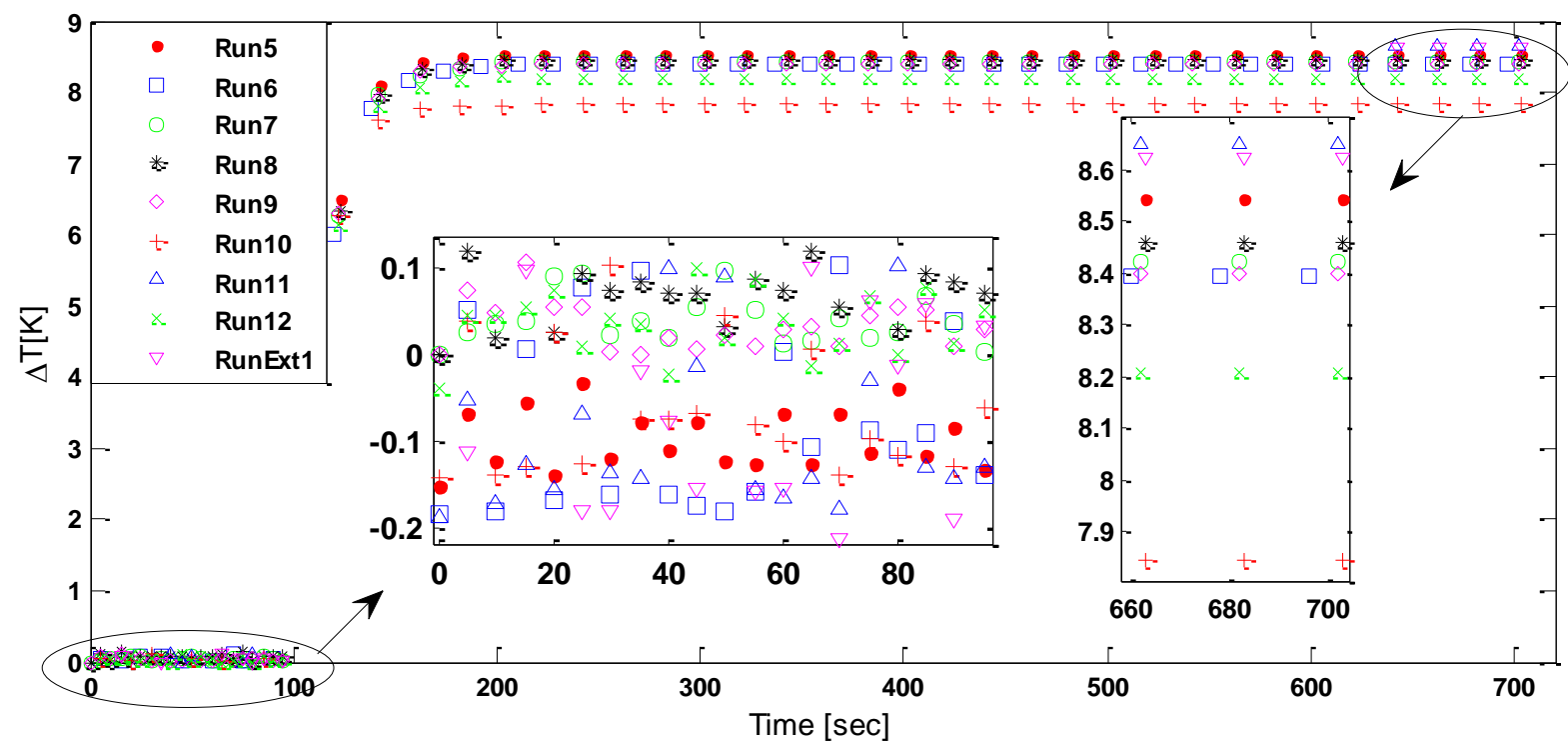

Figure 5-5: Temperature difference between hot and cold sides during thermal time

In Figure 5-6, the temperature variations along the height (thermal gradient direction) and at the middle of the cavity that correspond to different times during thermal time period are shown. The data for Run11 and Run12, which have the highest and the lowest Rayleigh vibration number, respectively. Although after the thermal time there is a noticeable curvature in the variation of temperature due to a single convection cell in the cavity, it is approximately linear after the thermal time for both runs. These trends show that a high Rayleigh vibration numbers, 1392 for example, does not cause a noticeable deviation from a case with a low Rayleigh vibration number, such as 1.3. Finally, after a short period of time, less than 2 minutes, and the temperature distribution is identical to the distribution at the end of thermal time. Figure 5-7 and Figure 5-8 illustrate the temperature field inside the IVIDIL cell during Run11 and Run12 at different times. Clearly, similar contours for both cases result from different times. In addition, an approximate linear variation in the temperature between the cold and hot sides was also seen. 


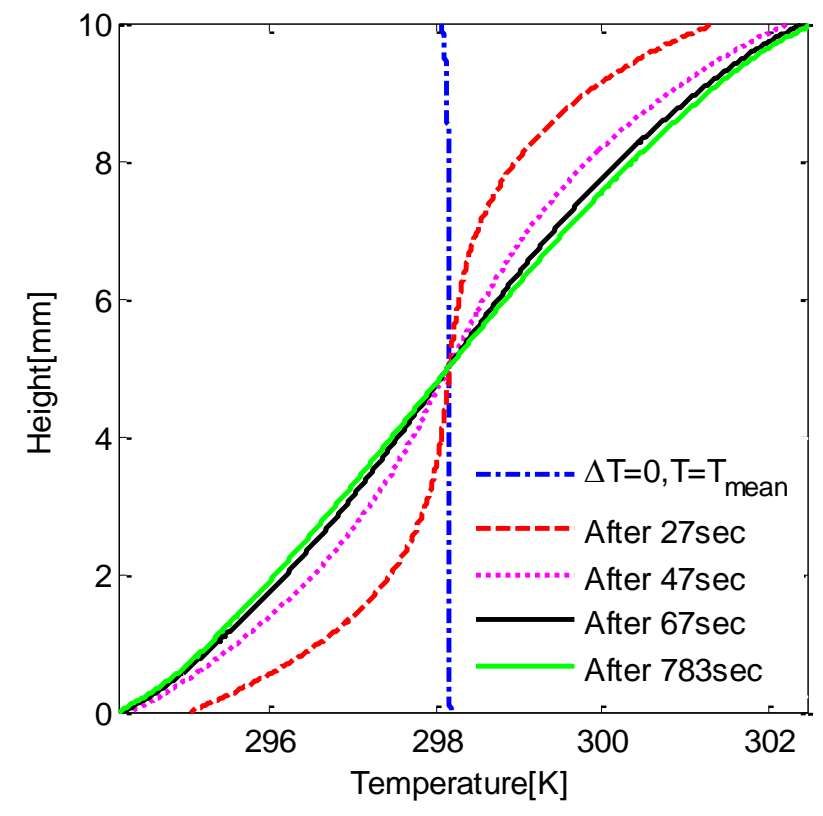

(a)

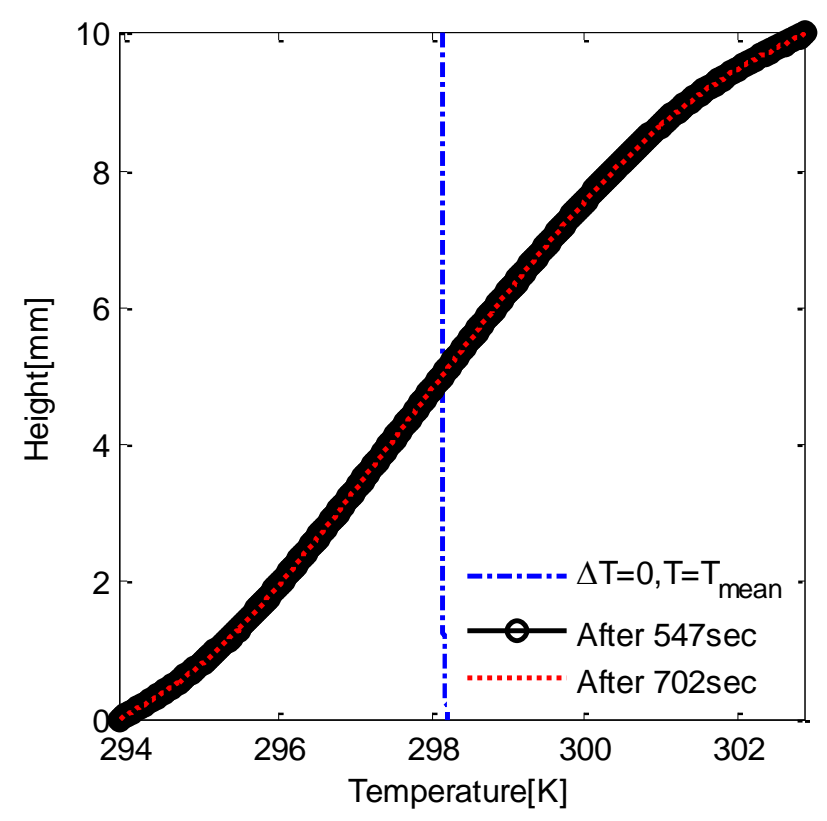

(b)

Figure 5-6: Temperature variation between hot and cold walls of a) Run12 and b) Run11

The thin gaps between the quartz glass and both the cold and hot walls of the cell set up cause a non-linearity of various contours originating at the corners of the cavity. Particularly, in the internal side of the O-rings, the small gap between copper and quartz is filled by the working liquid. On the other hand, the external side of the gap is filled by the surrounding gas that provides too large heat transfer $[66,109,131]$.
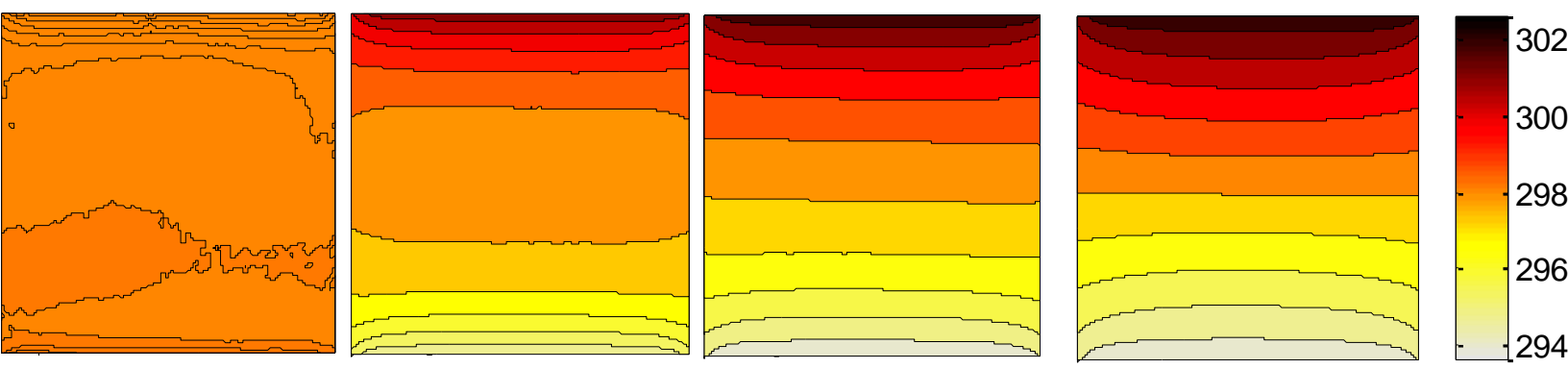

$\mathrm{t}=0[\mathrm{~s}]$

$\mathrm{t}=27[\mathrm{~s}]$ $\mathrm{t}=783[\mathrm{~s}]$

Figure 5-7: Temperature distribution in the cavity at different time during thermal time, Run12 Consequently, the largest thermal gradient arose at the corner of the cell due to the improper heat conductivity of the rubber seal and nearby regions (see Figure 5-7 and Figure 5-8). 


\subsubsection{Diffusion Time and Concentration Profile}

Figure 5-9 shows the concentration distribution of the test mixture versus time for all cases. Following from Figure 5-10, the concentration differences for all runs increased in a similar trend when the temperature stabilized.
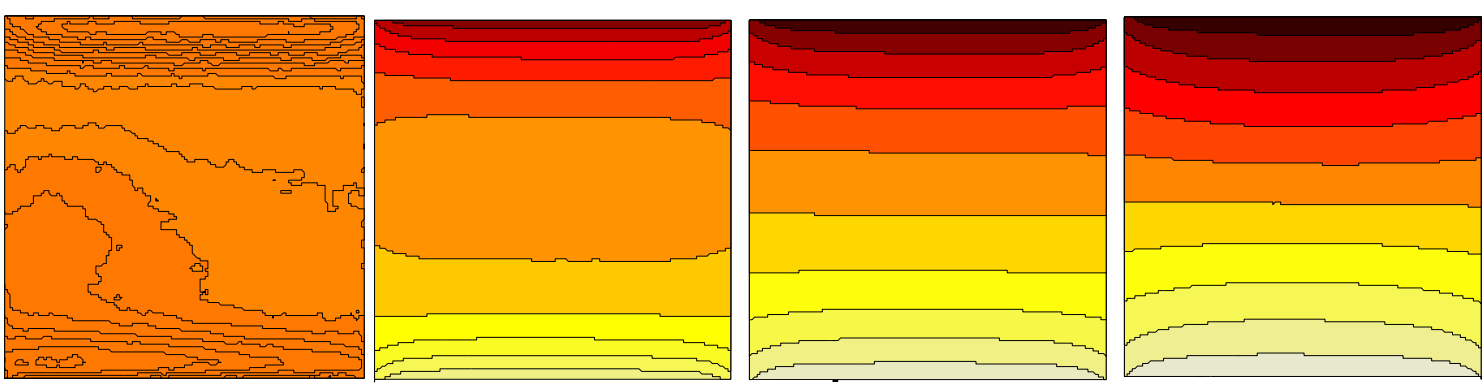

$\mathrm{t}=0[\mathrm{~s}]$

$\mathrm{t}=27[\mathrm{~s}]$

$\mathrm{t}=47[\mathrm{~s}]$

$\mathrm{t}=783[\mathrm{~s}]$

Figure 5-8: Temperature distribution in the cavity at different time during thermal time, Run11

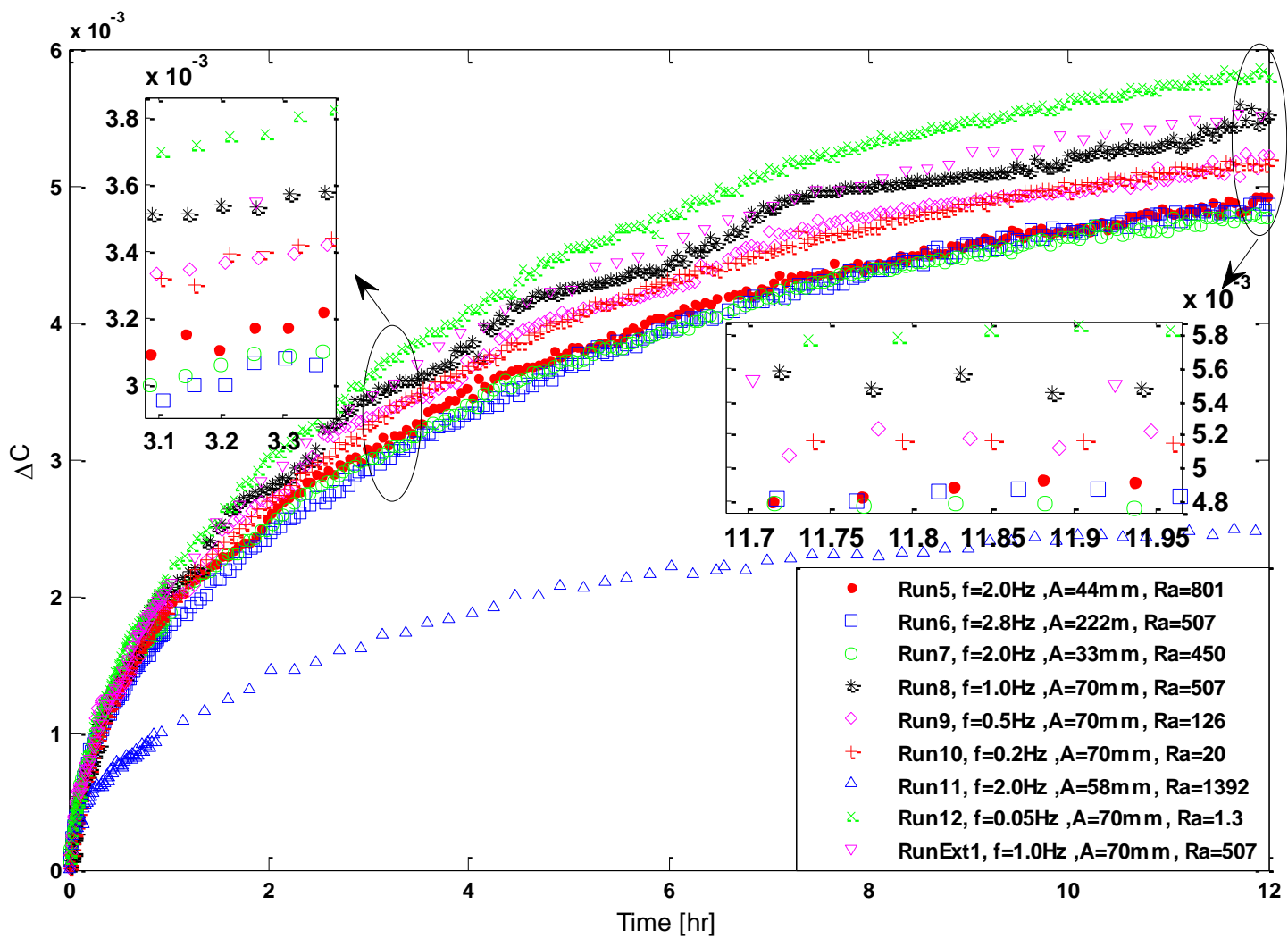

Figure 5-9:Separation between hot and cold sides during thermo-diffusion time 
The highest concentration difference or the Soret separation, corresponds to Run 12, which had the lowest vibration. Consistently, Run11, which had the highest vibration level, displayed the minimum Soret separation. A smooth pattern of separation versus time was recorded for all runs, and it corresponds well to the analytical solution in the convection-free regime. Nearly identical separations were observed for RunExt1 and Run8, which have distinct experimental conditions such as vibration characteristics and temperature gradients (The only difference is a longer period of thermodiffusion phase for RunExt1 that last $22 \mathrm{~h}$ ). This demonstrates the repeatability and accuracy of digital optical interferometry to calculate diffusion coefficients and to capture the smooth and precise trends of separation versus time. Generally, as the vibrational Rayleigh number increases the separation decreases. In other words, disturbances created due to higher Rayleigh vibrations caused less separation in the mixture than was observed for runs with lower Rayleigh vibrations.

Table 13: The IVIDIL experimental results with 10K temperature gradient onboard ISS

\begin{tabular}{|c|c|c|c|c|c|c|c|c|c|c|}
\hline RUN\# & $\begin{array}{c}\mathrm{c}_{\mathrm{o}} \\
\mathrm{IPA}\end{array}$ & $\begin{array}{c}\mathrm{f} \\
{[\mathrm{Hz}]}\end{array}$ & $\begin{array}{c}\mathrm{A} \\
{[\mathrm{mm}]}\end{array}$ & $\mathrm{Ra}_{\text {vib }}$ & $\mathrm{Ra}_{\text {vibexp }}$ & $\begin{array}{c}\Delta \mathrm{C}_{\max } \\
/ 10^{-3}\end{array}$ & $\begin{array}{c}\Delta \mathrm{T}_{\max } \\
{[\mathrm{K}]}\end{array}$ & $\begin{array}{c}\mathrm{S}_{\mathrm{T}, \mathrm{GA}} \\
/ 10^{-3} \\
{\left[\mathrm{~K}^{-1}\right]}\end{array}$ & $\begin{array}{c}D / 10^{-10} \\
{\left[\mathrm{~m}^{2} \mathrm{~s}^{-1}\right]}\end{array}$ & $\begin{array}{c}\mathrm{S}_{\mathrm{T}, \mathrm{st}} \\
/ 10^{-3} \\
{\left[\mathrm{~K}^{-1}\right]}\end{array}$ \\
\hline Ideal & $10 \%$ & --- & --- & 0 & 0 & $6.02 \pm 0.2$ & $8.30 \pm 0.1$ & $-8.47 \pm 1.5$ & $7.11 \pm 0.3$ & --- \\
\hline Run 12 & $10 \%$ & 0.05 & 70 & 1.3 & 0.9 & $5.87 \pm 0.2$ & $8.21 \pm 0.1$ & $-8.30 \pm 0.3$ & $6.71 \pm 0.3$ & 7.8 \\
\hline Run 10 & $10 \%$ & 0.2 & 70 & 20.3 & 12.5 & $5.23 \pm 0.2$ & $7.85 \pm 0.1$ & $-7.28 \pm 0.3$ & $7.38 \pm 0.3$ & 7.2 \\
\hline Run 9 & $10 \%$ & 0.5 & 70 & 126.8 & 89.4 & $5.23 \pm 0.2$ & $8.40 \pm 0.1$ & $-6.73 \pm 0.3$ & $7.03 \pm 0.3$ & 6.5 \\
\hline Run 7 & $10 \%$ & 2.0 & 33 & 450 & 319.6 & $4.81 \pm 0.2$ & $8.42 \pm 0.1$ & $-6.42 \pm 0.3$ & $7.26 \pm 0.3$ & 6.3 \\
\hline Run 8 & $10 \%$ & 1.0 & 70 & 507 & 378.5 & $5.53 \pm 0.2$ & $8.46 \pm 0.1$ & $-7.63 \pm 0.3$ & $5.64 \pm 0.3$ & 7.2 \\
\hline RunEx1 & $10 \%$ & 1.0 & 70 & 507 & 376.8 & $5.53 \pm 0.2$ & $8.62 \pm 0.1$ & $-7.64 \pm 0.3$ & $5.22 \pm 0.3$ & 6.2 \\
\hline Run 6 & $10 \%$ & 2.8 & 25 & 507 & 356.9 & $4.88 \pm 0.2$ & $8.39 \pm 0.1$ & $-6.55 \pm 0.3$ & $6.82 \pm 0.3$ & 6.3 \\
\hline Run 5 & $10 \%$ & 2.0 & 44 & 801 & 584.4 & $4.92 \pm 0.2$ & $8.54 \pm 0.1$ & $-6.59 \pm 0.3$ & $5.87 \pm 0.3$ & 6.4 \\
\hline Run 11 & $10 \%$ & 2.0 & 58 & 1392 & 1041.9 & $2.48 \pm 0.2$ & $8.65 \pm 0.1$ & $-3.44 \pm 0.3$ & $5.89 \pm 0.3$ & 3.1 \\
\hline
\end{tabular}

In order to compare the effect of Rayleigh vibrations, the concentration variations along the thermal gradient at the middle of the cavity during the diffusion period of the four runs were plotted in Figure 5-10.

Despite the small curvature that deviates from linear in Run12 after 12 h, (see Figure 5-10d), in general across all runs the linearity demonstrates that the ISS provides a suitable environment for this experiment. It must be added that in order to obtain a linear variation, the experiment needed more time than was given for separation phase in the condition of zero gravity when $g$-jitter vibrations are minimized during the experiment. 


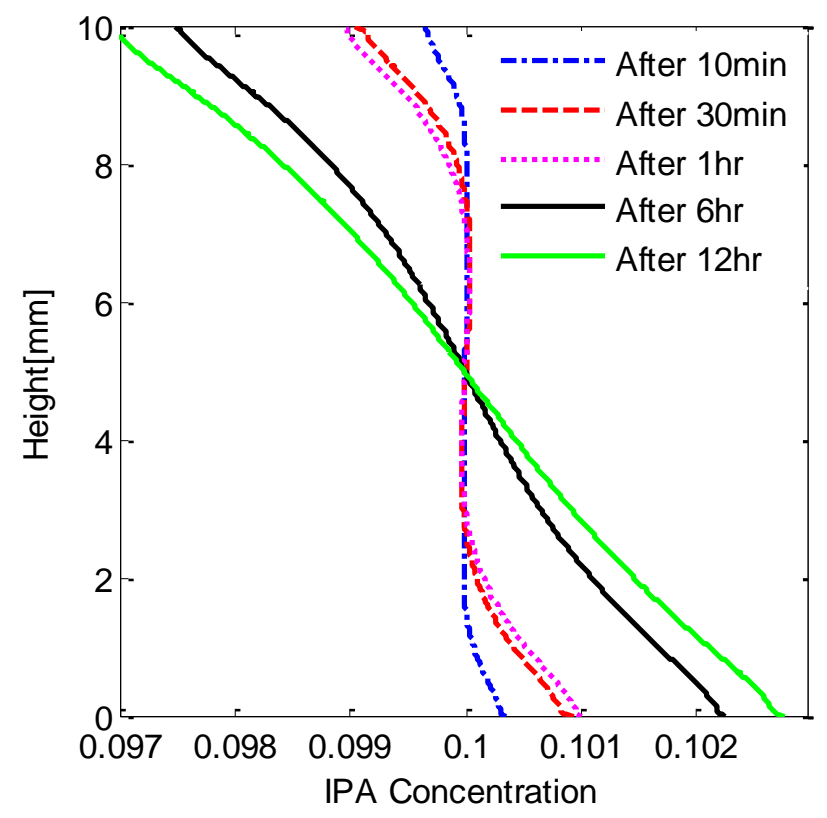

(a) Run12

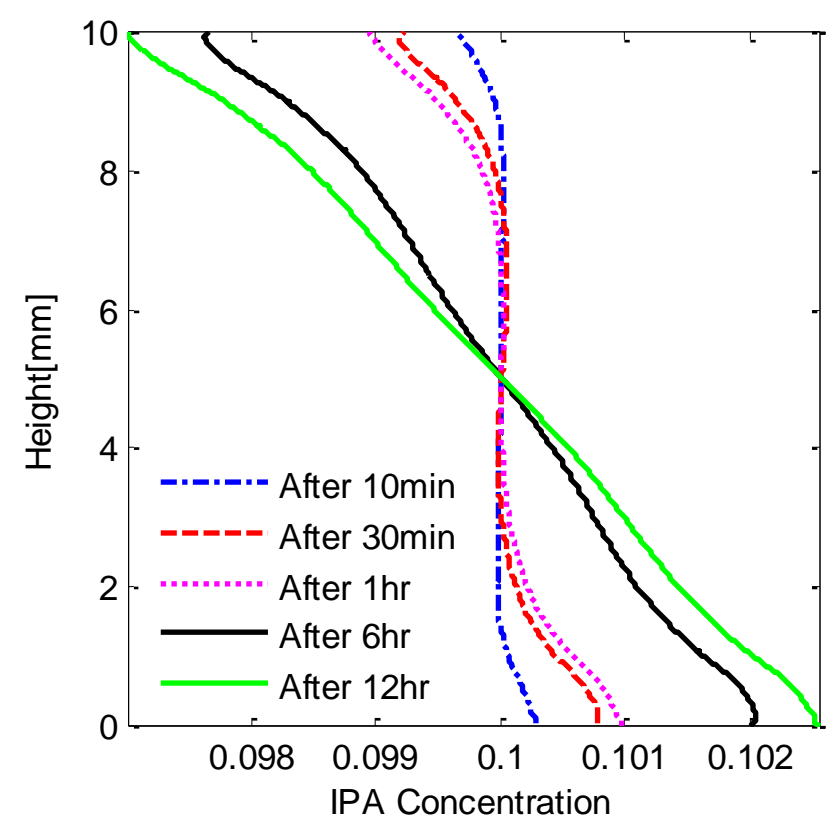

(c) Run8

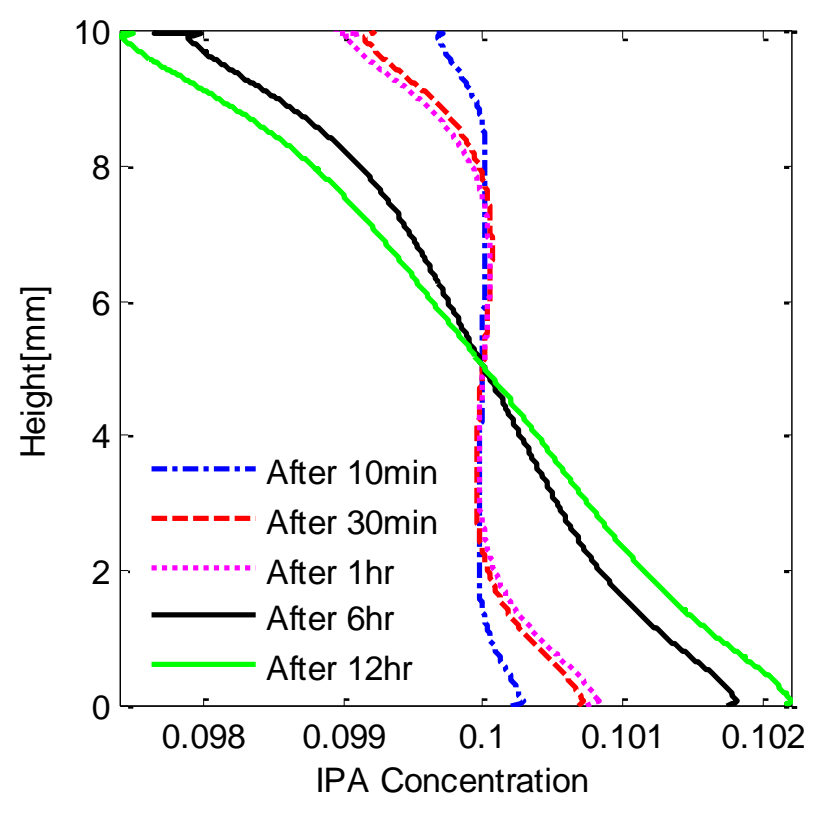

(b) Run5

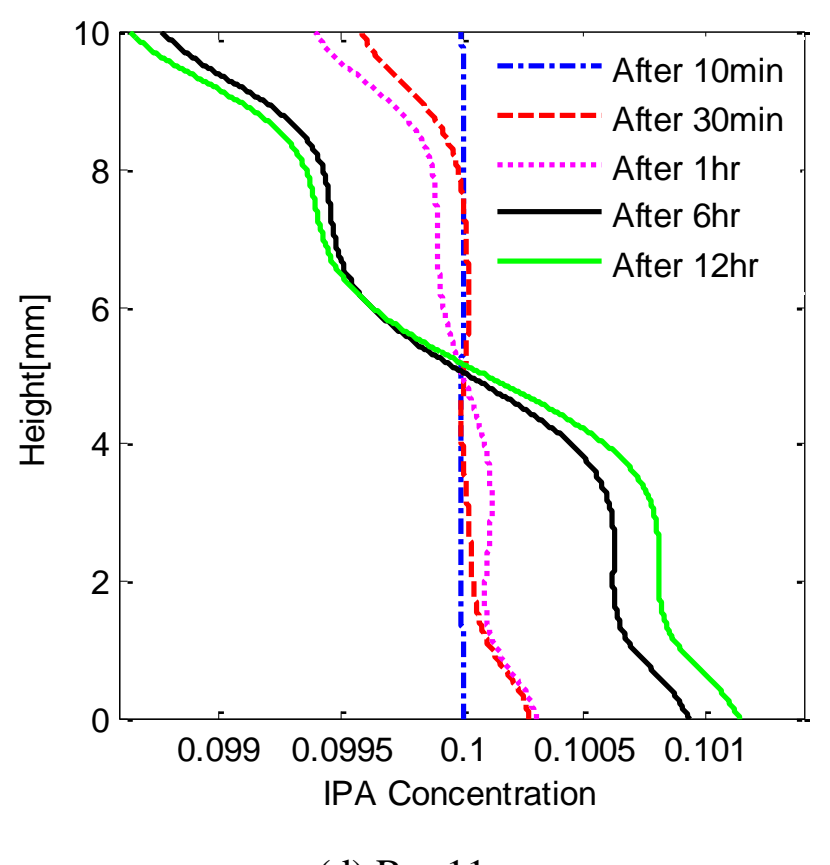

(d) Run11

Figure 5-10: IPA concentration variation between hot and cold walls at various times Approximate linear variations at the end of the diffusion time predict a smooth environment that behaves like a pure diffusion process. It is clear in all parts of Figure 5-10 that separation starts near both the cold and hot walls, while over time the separation also occurs far from the 
boundaries. When the Rayleigh vibration increases from 1.3 to 507 or 801 , the separation curvature increases (see Figure 5-10b, c) up to Run11 where it reaches the maximum curvature (see Figure 5-10d).

Another important observation is the large amount of separation (about 30 percent of the final separation) during the first $30 \mathrm{~min}$ of the thermal phase for most of the runs, with Run11 being an exception due to its high level of vibration. This behavior shows the importance of considering a precise estimate of the thermal time. In other words, an unsuitable value for thermal time may cause a large error in the measurement of the separation of components. As illustrated in Figure 5-10d, the separation trend of Run11 along the thermal gradient was different than the other cases. This difference suggests the existence of more than one strong convection cell in the cavity. These convection cells mix the liquid mixture inside the cavity and, as a result, homogeneous concentration regions can be observed along the cavity height. Thus, it must be concluded that while high Rayleigh vibration may not cause a deviation from linear temperature variation from the zero gravity condition, this vibration can overpower a pure separation pattern in the cavity.
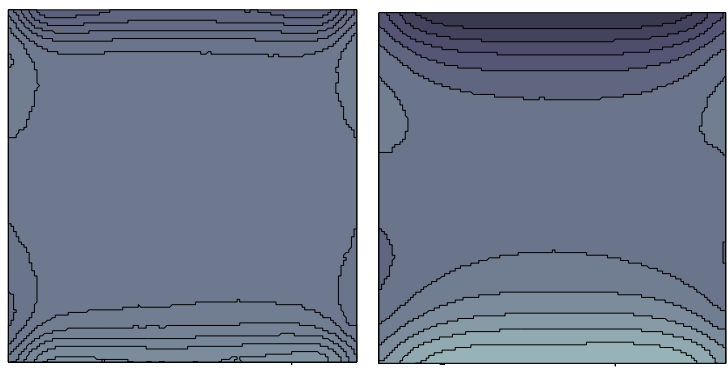

$\mathrm{t}=10[\mathrm{~min}]$

$\mathrm{t}=30[\mathrm{~min}]$

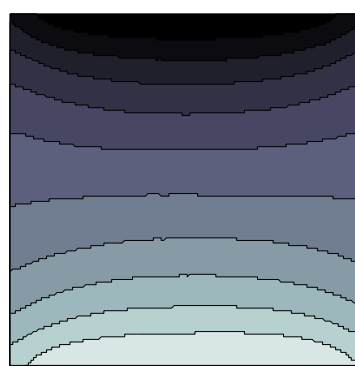

$\mathrm{t}=1[\mathrm{hr}]$
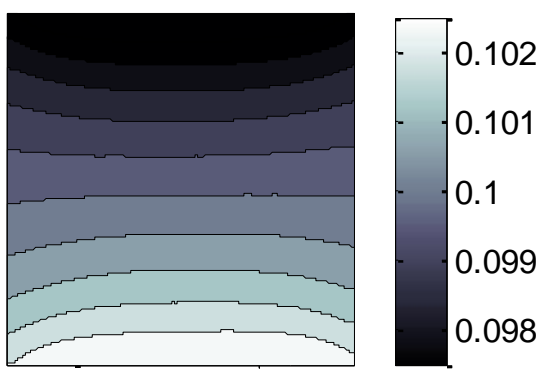

$$
\mathrm{t}=12[\mathrm{hr}]
$$

Figure 5-11: IPA concentration profile in the cavity at different time during diffusion time, Run12

Figure 5-11 and Figure 5-12 show the concentration contours inside the IVIDIL cell during Run12 at various times and at the end of the thermodiffusion phases of Run8, RunExt1, Run9, and Run11, respectively. Studying the remarkable effects of vibration is impossible if only considering the concentration variation along just one line at the middle of the cavity. Therefore, different contour cross-sections are required for studying the various cases. Though the 
separation originates close to both the hot and cold walls, it is interesting to note that the separation starts from two lateral walls as well. These walls are supposed to provide an adiabatic condition. This phenomenon can be seen during the first $30 \mathrm{~min}$ of the diffusion time (see Figure 5-11a). This occurred as a result of heat flux that existed close to the corners of the cavity due to a poorly designed isolation setup. While the separation in the domain becomes more pronounced after about one hour, it diminishes and then the concentration distribution follows the same pattern as the temperature contours. As mentioned in before, the temperature contours show a steep temperature gradient close to the corners of the cavity because of small gaps at the corners. Thus, these temperature variations affect the concentration pattern in the cavity mainly at the corners at the end of the thermodiffusion phase.
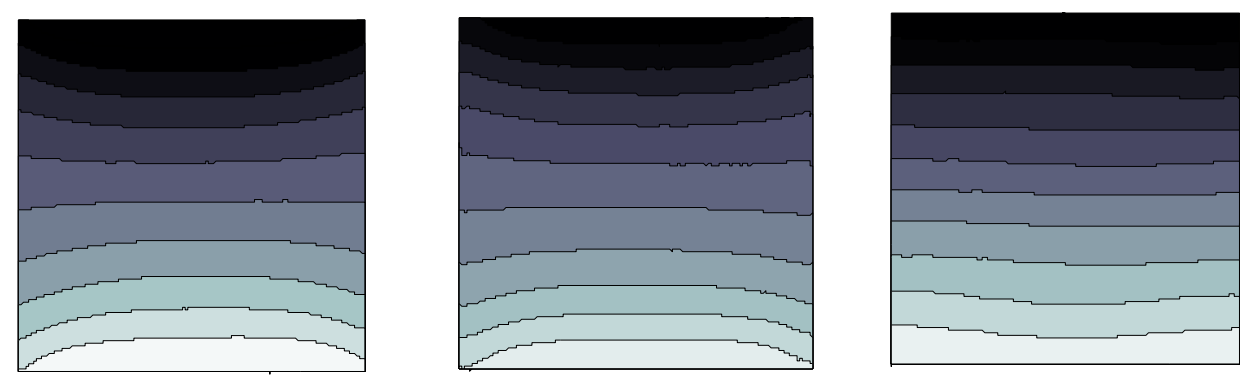

(a) Run12

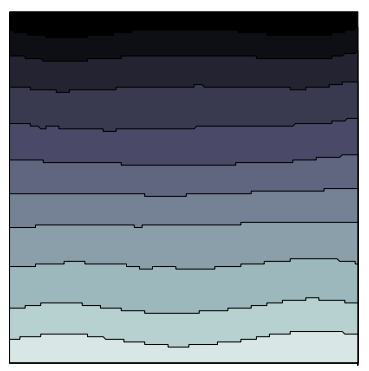

(b) Run9

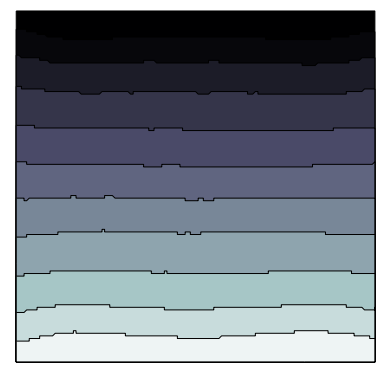

(e) Run5 (c) Run8

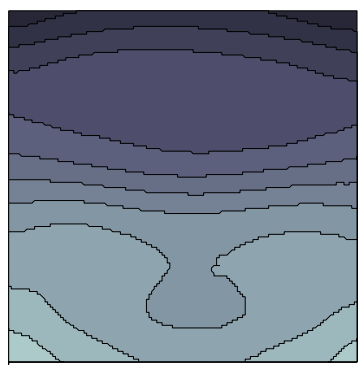

(f) Run11

(d) Run Ext1

Figure 5-12: IPA concentration profile in the cell for various runs at the end of thermodiffusion phase

It can be seen in Figure 5-12a that the similar temperature and concentration patterns between the hot and cold walls can be observed clearly in the domain for runs with low Rayleigh numbers 
(Run12, Run10 and Run9), which show higher separation at the middle of the cavity. Other cases that have higher levels of vibration, the vertical line with the maximum separation migrates towards the sides of the cavity, which means that close to the corners of the cell higher concentrations of components exist. The strong induced velocity field that was caused by at least one strong convection cell in the cavity mixed the liquid, at high $\mathrm{Re}_{\mathrm{vib}}$. This convection had its maximum effect on Run11, in which diffusion patterns were destroyed by more than one strong convection cell. These influences are compared versus various levels of controlled vibration in Figure 5-12.

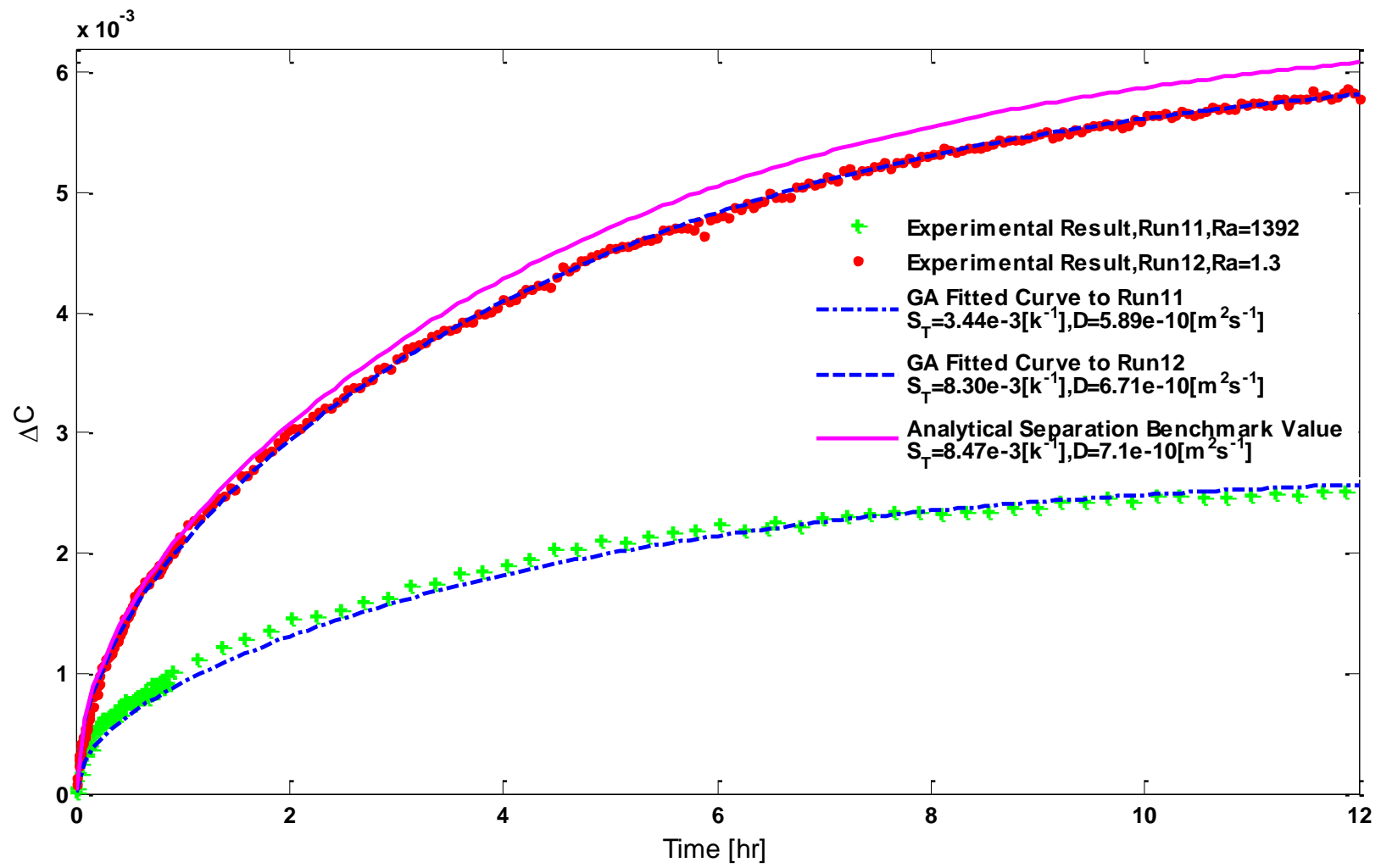

Figure 5-13: Analytical solution and maximum experimental separation during thermo-diffusion time, Runs11 and 12

\subsubsection{Soret Coefficient, $S_{T}$}

In contrast with cases without force vibrations, there are few analyses done in cases in which vibration is applied to the system. Nonetheless, Soret separations for cases with different Rayleigh vibrations were compared together with benchmark values of separation in this study. As described in Table 13, when the Rayleigh vibration number increases, the Soret seprations 
drops. Detailed discussion about the effects of different controlled vibrations requires having firm parameters for all cases that consider the influence of different temperature gradients and initial conditions. Otherwise, it would be hard to explain some of the exceptions that were recorded and correspond to the final separation after $12 \mathrm{~h}$. These behaviors are in contrast with the principle that shows the separation process decreases by increasing the level of controlled vibration as shown in Table 13.

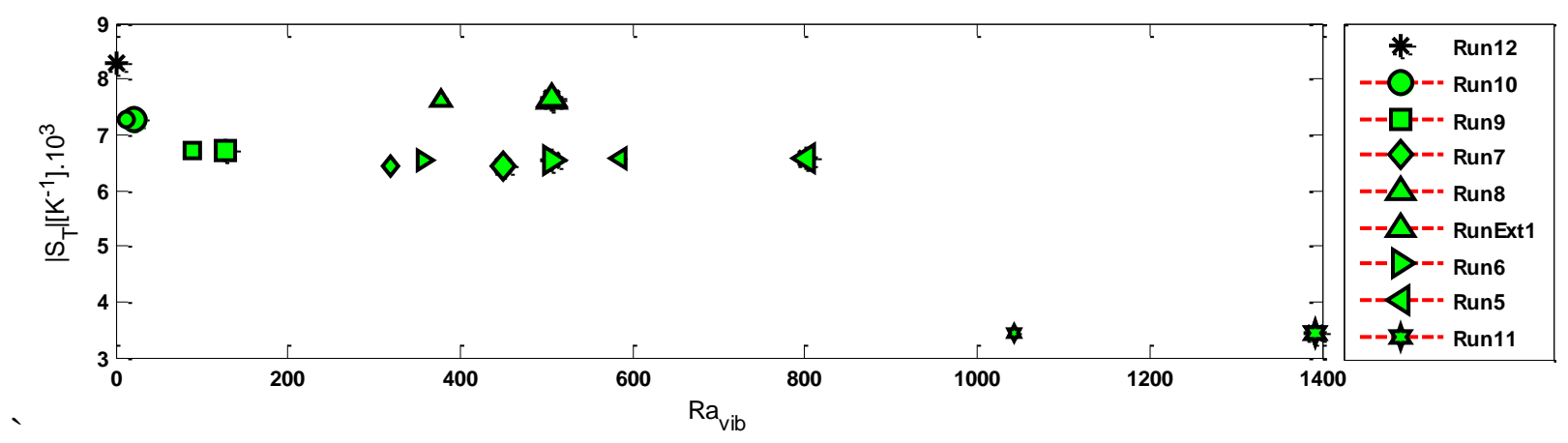

Figure 5-14: Soret coefficient versus Rayleigh number for all nine runs, the bigger symbols shows the results obtained by the thermocouple; while, the result of optical measurement illustrated by smaller symbols

There are few exceptions for this general behavior that need more discussion. For instance, two runs with different Rayleigh numbers that have similar separation. Run9, which was subjected to vibration of a Rayleigh number equal to 126, had the same value of separation as Run10, which had vibration with a Rayleigh number of 20 . The reason for this similarity, as summarized in Figure 5-5 and Table 13, depends on where the maximum temperature differences for these two runs occur. Run9, with higher Rayleigh number, had a larger temperature gradient causing more separation in the cavity. It is clear that a larger temperature gradient leads to greater separation at the end of the process, while it is difficult to explain the difference between the recorded maximum temperature gradient for each run. Consequently, considering only the maximum value of the separation after $12 \mathrm{~h}$ as the main parameter to evaluate the strength of separation between two runs is not sufficient. In the condition of zero gravity, comparing Soret coefficients is a precise parameter to show the strength of separation for runs with different characteristics. Thus the Soret coefficients, which are estimated for different runs, were used to compare with the theoretical value of the Soret coefficient for these mixtures, as shown in Table 11. 
According to the estimated Soret coefficients for Runs9 and Run10, which are presented in Table 13, a higher value of this coefficient for Run10 is strong evidence of higher level of separation in this run and a lower level of vibrational Rayleigh number compared to Run9. An exception to the negative effect of vibration on the diffusion process was seen in the experiments for Run8 and RunExt1, which manifest in a nonlinearity, as shown in Figure 5-14. As shown, despite the negative slope, which represents the negative effect of vibration on the separation, the data points of these two runs show a noticeably higher value of separation in comparison with other nearby data points. Another observation from this figure is the potential for employing the experimental temperature in calculating the Rayleigh vibration number. In Figure 5-14, dashed lines represent the nominal Rayleigh vibration number based on a $10 \mathrm{~K}$ temperature difference.

According to Gershuni theory, when the $\mathrm{Ra}_{\mathrm{vib}}$ is less than 10 the system is governed by pure diffusion. This behaviour can be seen for Run12 where $\mathrm{Ra}_{\text {vib }}=1.3$. As shown in Figure 5-13, the analytical solution using the benchmark value of $S_{T}$ for this mixture reveals a maximum variation of less than $5 \%$ between Run12 and the benchmark value. The analytical results of separation were found through a numerical approach for runs with forced vibration. Further numerical simulations and analyses of the effect of forced vibration can found in literature $[63,64,112]$. According to these numerical simulations, it was found that the magnitude of the induced velocity in such a cell is around 5 to $10 \mu \mathrm{m} / \mathrm{s}$, which corresponds to various g-jitter conditions. When $\mathrm{Ra}_{\text {vib }}$ is greater than 10 , for instance $\mathrm{Run} 10$ with $\mathrm{Ra}_{\mathrm{vib}}=20$, a sudden decrement was evident in the curve of the maximum separation versus $\mathrm{Ra}_{\text {vib}}$, as shown in Figure 5-14. After this jump and as $\mathrm{Ra}_{\text {vib }}$ increases, the maximum separation decreases with a low slope until the second jump close to $\mathrm{Ra}_{\mathrm{vib}}=1 \times 10^{3}$. These behaviours are in complete agreement with Gershuni theory in various vibrational regimes. As shown, the effect of vibration was negligible up to Run10 (Gs 20) in which $95 \%$ of the separation of zero-gravity condition was achieved. Further increase in Gershuni number affected the diffusion process as induced convection dominated the diffusion process. However, it was found that when the Gershuni number is below 10, the process is completely governed by diffusion. Further increase of the Gershuni number triggers mixing effects [128,129]. Figure 5-13 represents the theoretical separation of the mixture versus the experimental separations measured for Run12 and Run11, which have the minimum and maximum Rayleigh numbers, respectively. As explained earlier, 
GA was used to calculate the Soret and diffusion coefficients for all runs. It was found that these curves coincide with their experimental data points, especially for Run12, which has a similar pattern to pure diffusion because of its low Rayleigh vibration number. These matched curves demonstrate the good performance of this method to estimate diffusion coefficients. It must be noted again that Run12, with very small vibrational Rayleigh number, behaves close to theory. In addition, the estimated Soret coefficient for this run is three percent less than its benchmark value, which indicates only a small influence on the separation at this low Raleigh vibration number and follows the vibration instability theories in the literature $[128,129]$.

It can be concluded from these two close values of the experimental and theoretical Soret coefficients that the results of the IVIDIL experiments are reliable and precise. As an exception, the separation for Run11 is more than $59 \%$ off from the analytical separation. This deviation is likely due to a destruction of the diffusion pattern by induced convection due to the high level of forced vibration. This methodology of estimating Soret coefficient was used for other runs and the results can be found in Table 3. It must be noted that the pattern of separations of Run5, Run6, and Run7 were close to each other. This similarity may have occurred because of the close vibration level ranges of these runs. While it was observed that Run5 had a higher separation than that of Run6 and Run7, which was expected as its vibration was highest of the three, this phenomenon requires more investigation as it may also have occurred as a result of an existing

high level of g-jitter vibration on board the ISS during Run6 and Run7. In all cases with Rayleigh vibrational numbers more than 126 , the concentration contours strongly deviate from a pure diffusion pattern because of high Rayleigh vibrations.

\subsection{Conclusion}

The goal of this research was achieved by determining the impacts of various selected forced vibration levels and fixed temperature gradients on the measurement of thermodiffusion. For this purpose, nine different runs of water and isopropanol with a negative Soret coefficient and consistent temperature differential were tested in a microgravity condition. Mathematical analyses by means of fast Fourier transform image processing and an advanced curve-fitting method using GA were employed to obtain the most accurate results. 
The results were compared with the analytical trends in the convection-free regime without any forced or g-jitter vibration using benchmark values of diffusion coefficients. Results revealed maximum separation for the case with the minimum Rayleigh number; however, a linear relationship between vibrational Rayleigh number and maximum separation was not found. The findings of this research can be summarized as follows: First, measurement of the temperature difference experimentally had a direct effect on the final results. This is seen by the considerable change of the temperature variation among different runs. In addition, substantial deviation of the thermodiffusion coefficient shows the importance of measuring temperature in the cavity experimentally. It was found that 12 hours is not enough to reach steady state for the separation of components in this particular mixture, as shown by the large amount of separation during the last hours of the experiments on board the ISS.

Second, as the Rayleigh vibration number increases, the separation decreases in most of the cases. The significance of considering the precise thermal time as a result of the high ratio of separation was discussed. Third, the Soret coefficient was found to decrease as the Rayleigh vibration number increases. It is worth noting that considering only the maximum value of the separation after 12 hours as a main parameter to evaluate the strength of separation between two runs is not sufficient when dealing with the small differences in temperature variations of the various runs in these experiments. In short, the ISS provides a suitable environment for this experiment, and the results of the IVIDIL experiments are reliable and can be used as reference material for ground based experiments. 


\section{Chapter 6: Experimental Study of the Impacts of Forced Vibration on Thermodiffusion Phenomenon}

\subsection{Introduction}

Different sources such as experiment operation, aerodynamic drag, gravity gradient, life-support systems, equipment operation, crew activities, and rotational effects create microgravity vibration on board vehicles in microgravity environments [64,111,133]. This microgravity vibration is one of the most important factors that can affect thermodiffusion experiments on board the ISS. It has been argued that controlled vibrations or g-jitter vibrations in a reduced gravity condition can reduce the separation of components in a mixture [7,32,134,135]. For this reason, the IVIDIL experiment investigated the influences of the force oscillatory vibrations on thermodiffusion phenomenon.

The uncertainties of the IVIDIL experiments have been discussed in different investigations. For instance, the effects of this vibration, which are recorded on board the ISS were studied in detail by Ahadi et al. $[63,64]$ and Shevtsova et al $[65,66]$ and it was found that at the specifics microgravity perturbations that are higher than critical value, the results of the experiment would be affected and would be deviated from the theoretical predicted results.

\subsection{Chapter Aims}

In the present chapter, the effect of forced vibration on the separation pattern using the experimental data of the IVIDIL project is investigated. Here, the eight experimental runs with a similar temperature difference and a mean temperature of $298 \mathrm{~K}$ were compared to determine the effects of vibration on the separation of the liquid mixtures. The experimental mixture was water (90\% wt.) and isopropanol $\left(\mathrm{C}_{3} \mathrm{H}_{7} \mathrm{OH}\right)$.

Data were received in the form of binary data. The received images must be processed and analyzed by different image processing techniques to extract the phase map. Next section briefly presents the experimental setup and the experimental procedure. The results and discussion are presented in the last section followed by the conclusions and remarks. In this chapter, the effects 
on a thermodiffusion experiment of increasing the Gershuni number (Gs) from zero to Gs $\sim 3 \mathrm{x}$ $10^{3}$ with a constant temperature gradient of $15 \mathrm{~K}$ were studied for the first time. While many investigations have been done studying thermodiffusion phenomena in a micro-gravity condition, and preliminary Discussion on IVIDIL objectives have been proposed, only a few works can be found in the literature that address the result of this project. However, results indicate a maximum separation and Soret coefficient for the case with a minimum Gershuni number, and different concentration profiles and separation patterns were observed. Remarkably, when Gs $=1.5 \times 10^{3}$, the maximum separation may not occur at the end of diffusion time.

Here, nine experimental runs were considered and analyzed in detail, summarized. These experimental runs were performed under identical temperature gradients $(\Delta T=15 \mathrm{~K})$, but different Gershuni numbers, from the lowest of 678 which corresponds to Run17, to the highest value of 3465 in Run15.

\subsection{Results and Discussion}

\subsubsection{Thermal Time and Temperature Profile}

Numerically, it has been shown that the low frequency vibrations produce a stronger impact on the flow and the maximal value of the temperature field from linear profile [136]. Thus, it has been derived that external vibrations that have a lower frequency than $0.1 \mathrm{~Hz}$ have a stronger effect on all quantities of the flow.

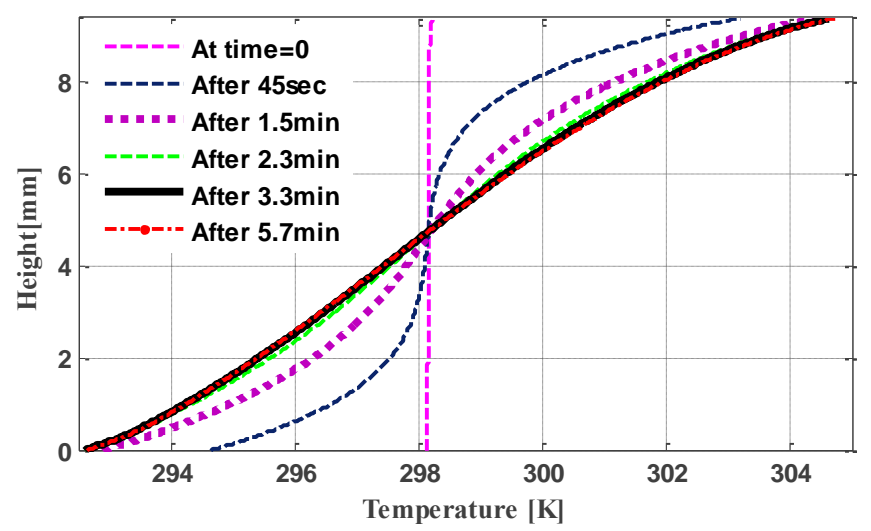

Figure 6-1: Temperature variation between hot and cold walls 
In this study, the minimum frequency is $1 \mathrm{~Hz}$ and most of the runs have a frequency of $2 \mathrm{~Hz}$, thus the actual temperature fluctuation should be less than $1 \times 10^{-5} \mathrm{~K}$. This means after thermal time it is accurate to assume a constant temperature profile in the cell. The properties of the liquids and their contrast factors at the mean temperature of $298 \mathrm{~K}$ can be found in Figure 6-1 for this mixture. These values were used in the calculation of the temperature field in the cavity at each pixel of the images, to produce with an error less than $0.05 \mathrm{~K}[110,112]$. A linear variation of the thermal gradient was reached in the cavity at the end of thermal time. According to the given relation for thermal time stated earlier, this parameter for the water-IPA mixture is more than 12 min. Figure 6-1 illustrates the temperature variations along the height (the thermal gradient direction) and at the middle of the cavity at different times during thermal time for Run15. Although after the thermal time the temperature variation along the temperature gradient direction can be observed as a result of heat fluxes at the walls. Note that according to Figure 6-1, after 3 min of applying the thermal gradient, $\Delta \mathrm{T}$ between the hot and cold walls had its maximum and/or steady value. While $\Delta \mathrm{T}$ between the heights of $2.5 \mathrm{~mm}$ and $7.5 \mathrm{~mm}$ showed a small change of temperature (less than $0.1 \mathrm{~K}$ ) during last 9 min of thermal time.

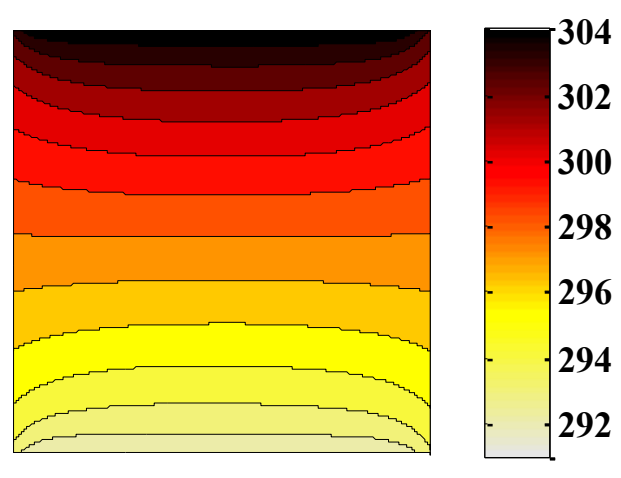

Figure 6-2: Temperature contour inside cell after thermal time

Figure 6-2 demonstrates the temperature contour inside the binary cell during Run15, which had the largest level of forced vibration. The similar temperature contour between this case and the run without vibration (Run2) suggests that the forced vibration in these cases does not affect the temperature variation in the domain. Because of the small thin gaps between the quartz glass and both the cold and hot walls of the cell setup, the non-linearity of temperature started from the corners of the cavity[110,112]. 


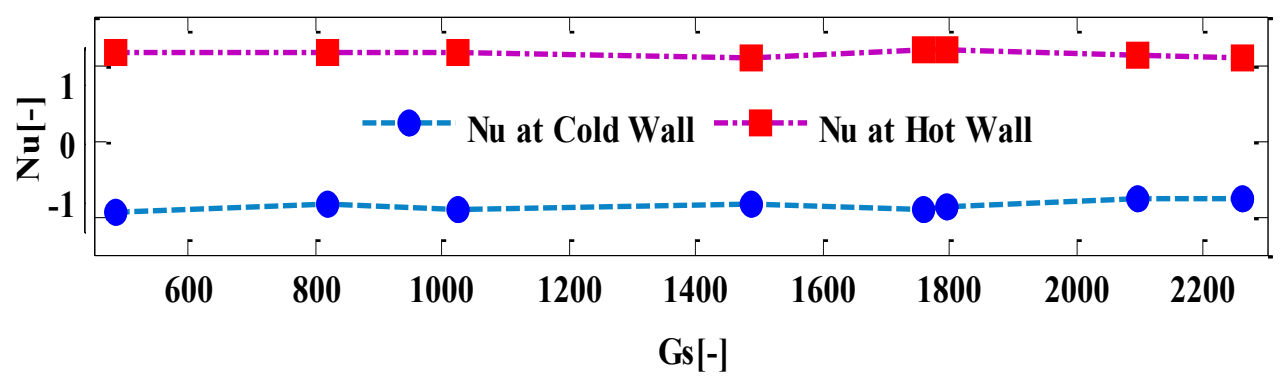

Figure 6-3: Average Nusselt number at the cold and hot walls

The maximum temperature differences of all runs between the two hot and cold walls are summarized in Figure 6-3. It is of note that that while more than $90 \%$ of the height of the cavity is considered for processing, the maximum and minimum temperature differences are $12.4 \mathrm{~K}$ and 12.7 K in Run15 and Run16, respectively. Although the temperature difference is supposed to be 15 , even in the case of considering our cropping size the temperature difference should still be larger than $13.5 \mathrm{~K}$. Consequently, the substantial deviation of ideal thermodiffusion noticed at the end of these runs, which was caused by the difference between the predicted $15 \mathrm{~K}$ temperature difference and the actual one (lower measured temperature difference than $15 \mathrm{~K}$ ). This shows the importance of measuring temperature in the cavity experimentally.

Finally, Figure 6-3 shows that the heat fluxes close to the hot and cold walls are similar in different directions (using the Nusselt number that is defined as Equation 6-1. However, as the vibration level increases, the convection becomes stronger, which causes a slight increase in the heat transfer at the walls. Although in this range of Gs, the heat transfer at the wall does not change significantly with an increase in Gs. The Nusselt number is slightly greater than one $(\sim 1.15)$ that points to a convection regime.

$$
N u=\frac{L}{2 \Delta T} \oint_{\Gamma}\left|\frac{\partial T}{\partial n}\right| d S
$$

\subsubsection{Diffusion Time and Concentration Profile}

The effect of convection that can be seen in Figure 6-4:, is that it causes the system to reach a steady-state condition faster. For example, Run17, which is the run with the weakest vibration, needed more than $12 \mathrm{~h}$ to reach steady state, while the time for reaching steady state for the run 
with the second weakest vibration (Run16) and the strongest vibration (Run15) decreased to10 $\mathrm{h}$ and $6 \mathrm{~h}$, respectively. It is also evident in Figure 6-4 : that the mass transport process for Run17 and Run16 were dominated by thermodiffusion, and for the other runs it is the convection in the system that dominated.

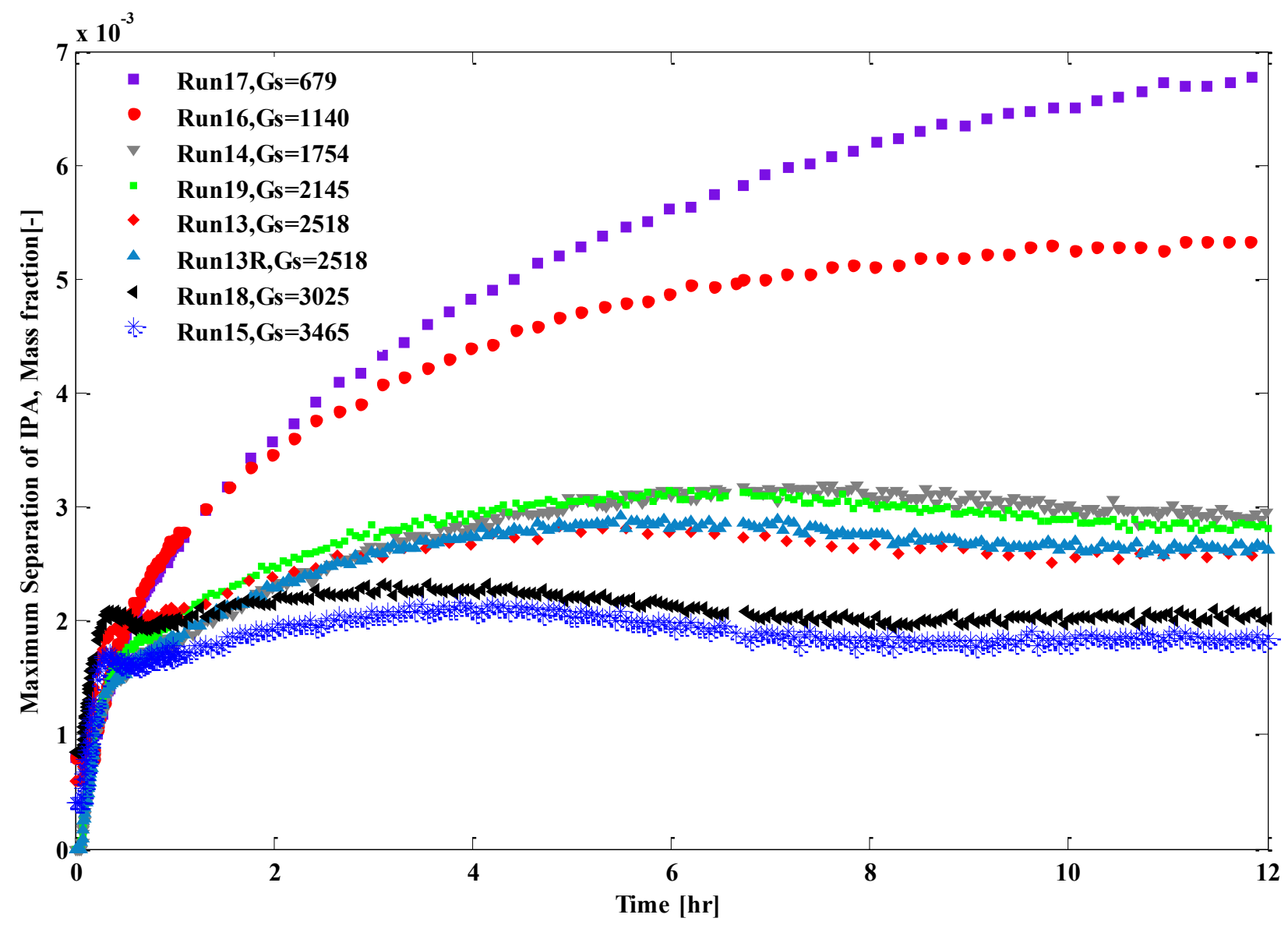

Figure 6-4 :Maximum Soret separation between walls

Figure 6-5: shows concentration variations along the thermal gradient direction at the middle of the cavity at several times during the diffusion period for four runs. Despite of the small curvature of Run17 after $12 \mathrm{~h}$, which deviated from the linear variation from the top to the bottom of the cavity (see Figure 6-5:a), the trend is completely non-linear for other runs. This remains true even for Run16 (shown in Figure 6-5:b), which has the closest vibration level to Run17. The influence of convection is evident in the concentration distribution in Run14; moreover, Run15 (Figure 6-5:d), which has the highest vibration, showed the strongest convection cell, which mixes the entire fluid inside the cavity. 


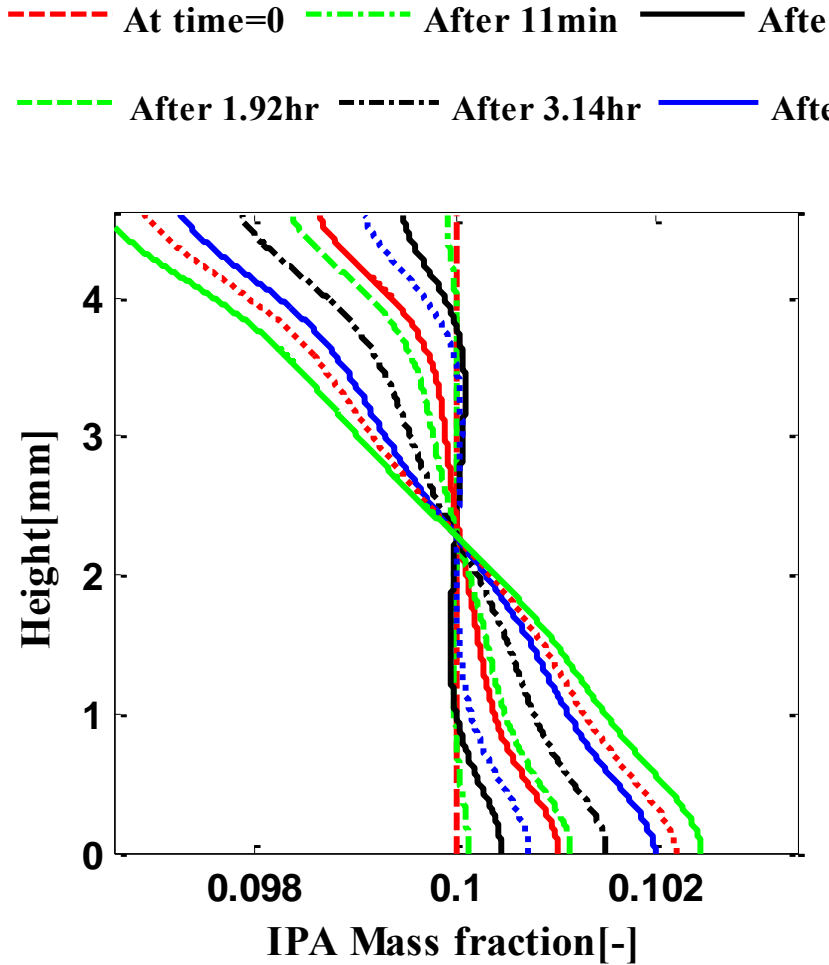

(a) Run17

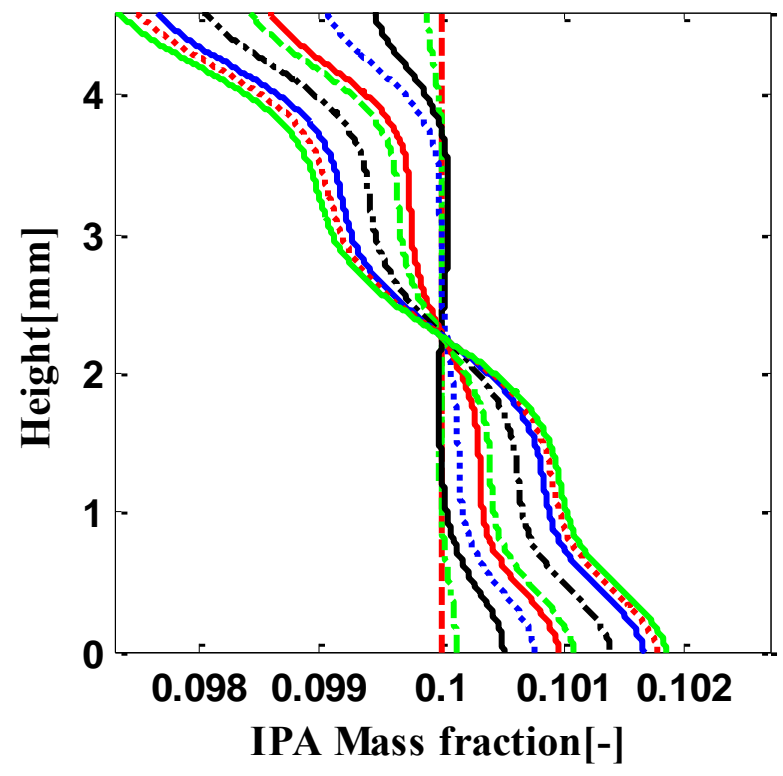

(b) Run16

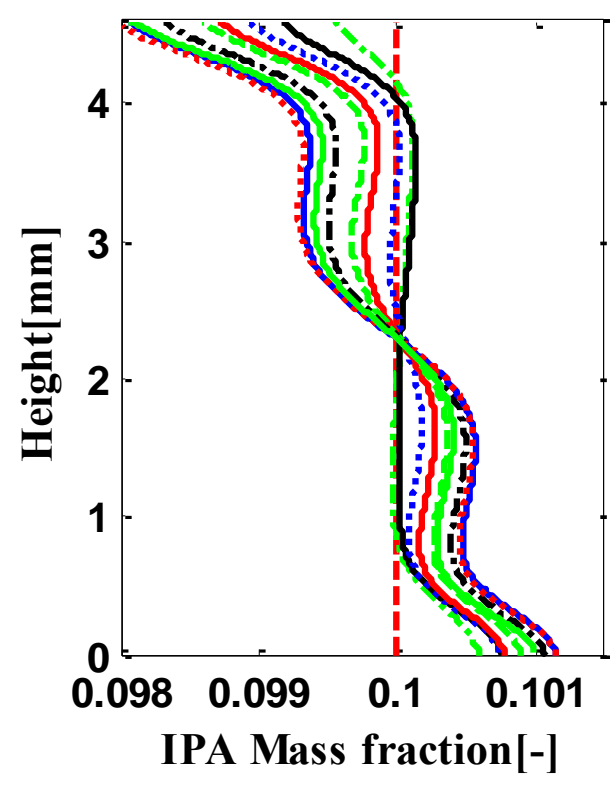

(c) Run14

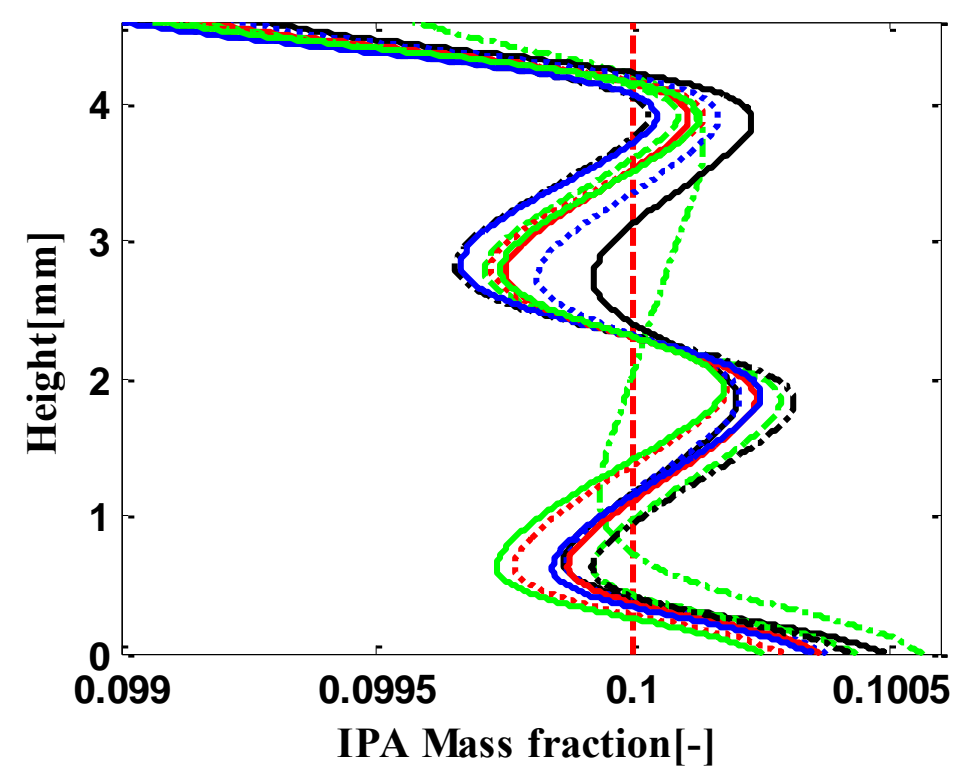

(d) Run15

Figure 6-5:IPA concentration variation at the middle and along temperature gradient direction at various times 


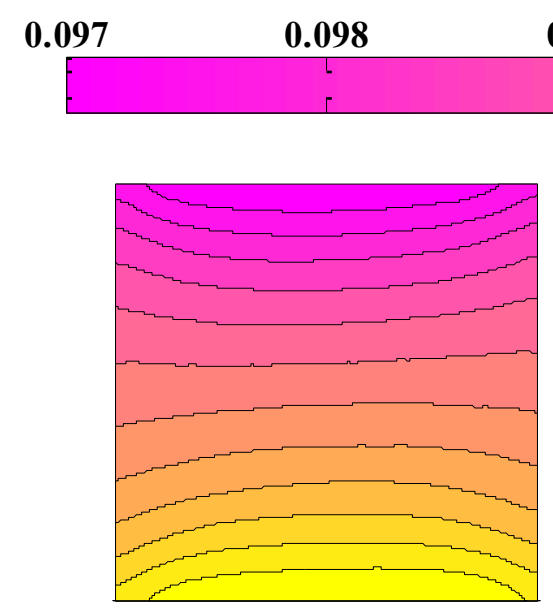

(a) Run12

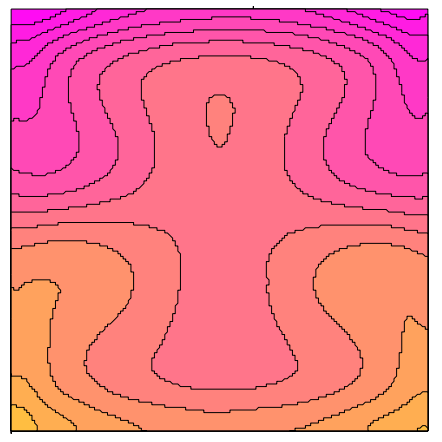

(d) Run14

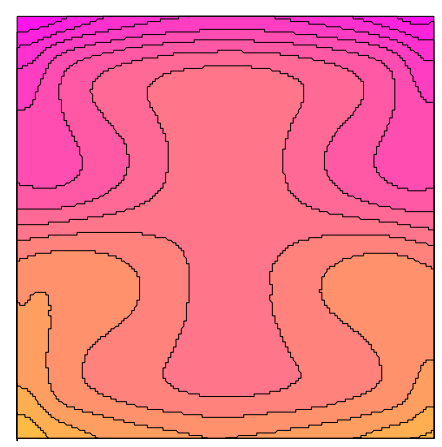

(g) Run13R

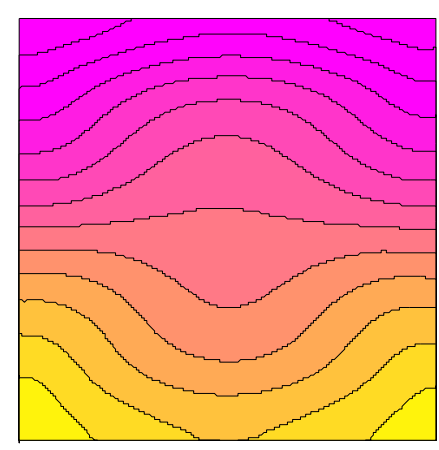

(b) Run17

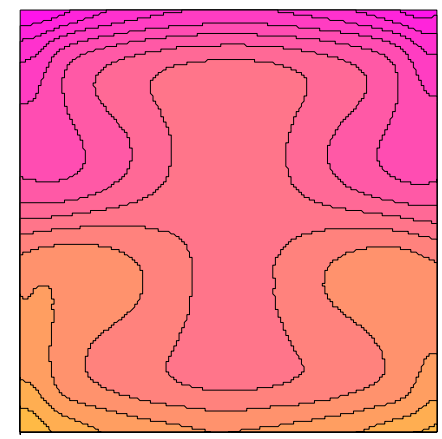

(e) Run19

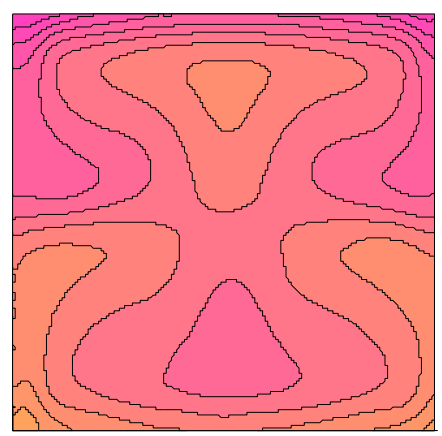

(h) Run 18

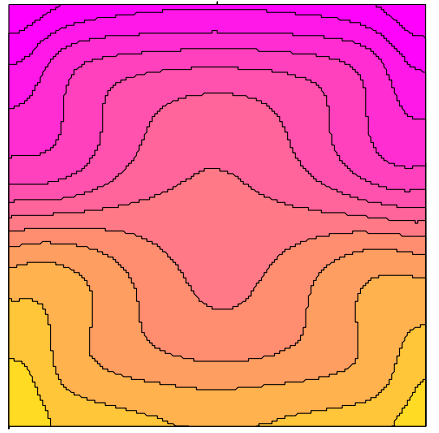

(c) Run16

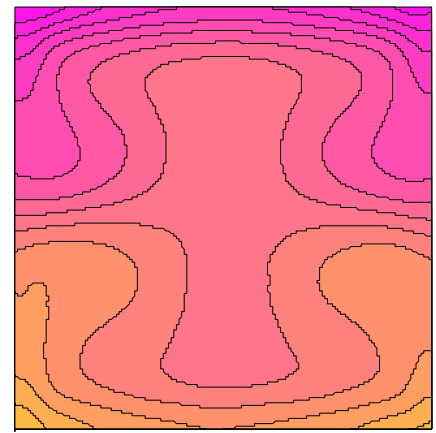

(f) Run13

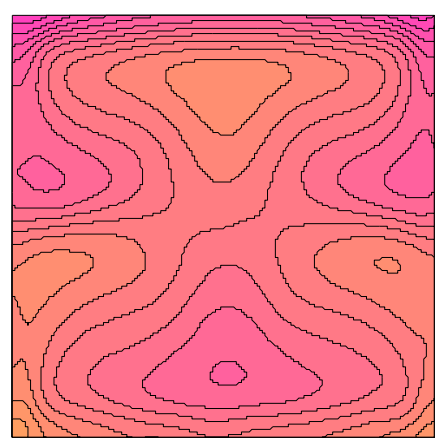

(i) Run15

Figure 6-6: IPA concentration profile inside cell for various runs after 12 hours 
It is remarkable that while points close to the hot and cold walls show a higher concentration of IPA and water, respectively, points nearby to these point at $\mathrm{z}=2 \mathrm{~mm}$ and $\mathrm{z}=8 \mathrm{~mm}$ show the opposite direction of separation with the same magnitude.

Studying the remarkable effects of vibration is impossible by considering concentration variation along just one line at the middle of the cavity. Thus, Figure 6-6 illustrates the concentration contours inside the primary IVIDIL cell during Run12, Run17, Run16, Run14, Run19, Run13, Run13R, Run18, and Run15 at the end of the thermodiffusion phase. While Figure 6-6a shows Run12 with a very small vibration as $\mathrm{Gs}=1.3$, it produced the same concentration contour as the temperature contour pattern. The curvature occurs as a result of heat flux that exists close to the corners of the cavity due to inadequate heat isolation in the equipment $[110,112]$.

Thus, the non-linear temperature profile affects the concentration pattern in the cavity mainly nearby the corners at the end of thermodiffusion phase. While for other cases with higher levels of vibration, the vertical line that contains the maximum separation of species migrates to the two corners of the cavity. It demonstrates that the strong, induced convection cells formed as vibration level increased. This observation has its maximum effect in runs15 and 18, in which the diffusion pattern was destroyed by more than one strong convection cell (four convection cells). These influences can be compared against various levels of controlled vibration in Figure 6-6. While strong mixing is apparent in all cases (except Run12), it can be seen that the Soret effect is dominant in the first three cases of Figure 6-6. Parts d, e, f, and g of Figure 6-6 illustrate a homogeneous concentration region at the center of the cell, and the last two parts of this figure show regions of high concentration of IPA close to the hot wall that occurs due to critical mixing in the cavity.

Note that the concentration profile is almost homogeneous for the last case, except at the walls where a high gradient of separation is noticeable. The same observations were found numerically [94]. With an increase in the vibration level the local maximum occurred earlier in time and its relative value was lower. This can be seen in Figure 6-7 and Figure 6-8. As illustrated in Figure 6-8, the steady-state values of the separations may be presented as a function of the oscillatory acceleration level. Moreover, Figure 6-7 shows the different mechanisms in mass transport in comparison with the pure thermodiffusion. A weak dependence of separation on 
vibration level was detected for Gs > 1000, which means that the mixing caused by vibration was at its maximum strength in this regime. Moreover, the relation between time of maximum separation and Gs number is shown in Figure 6-8 .

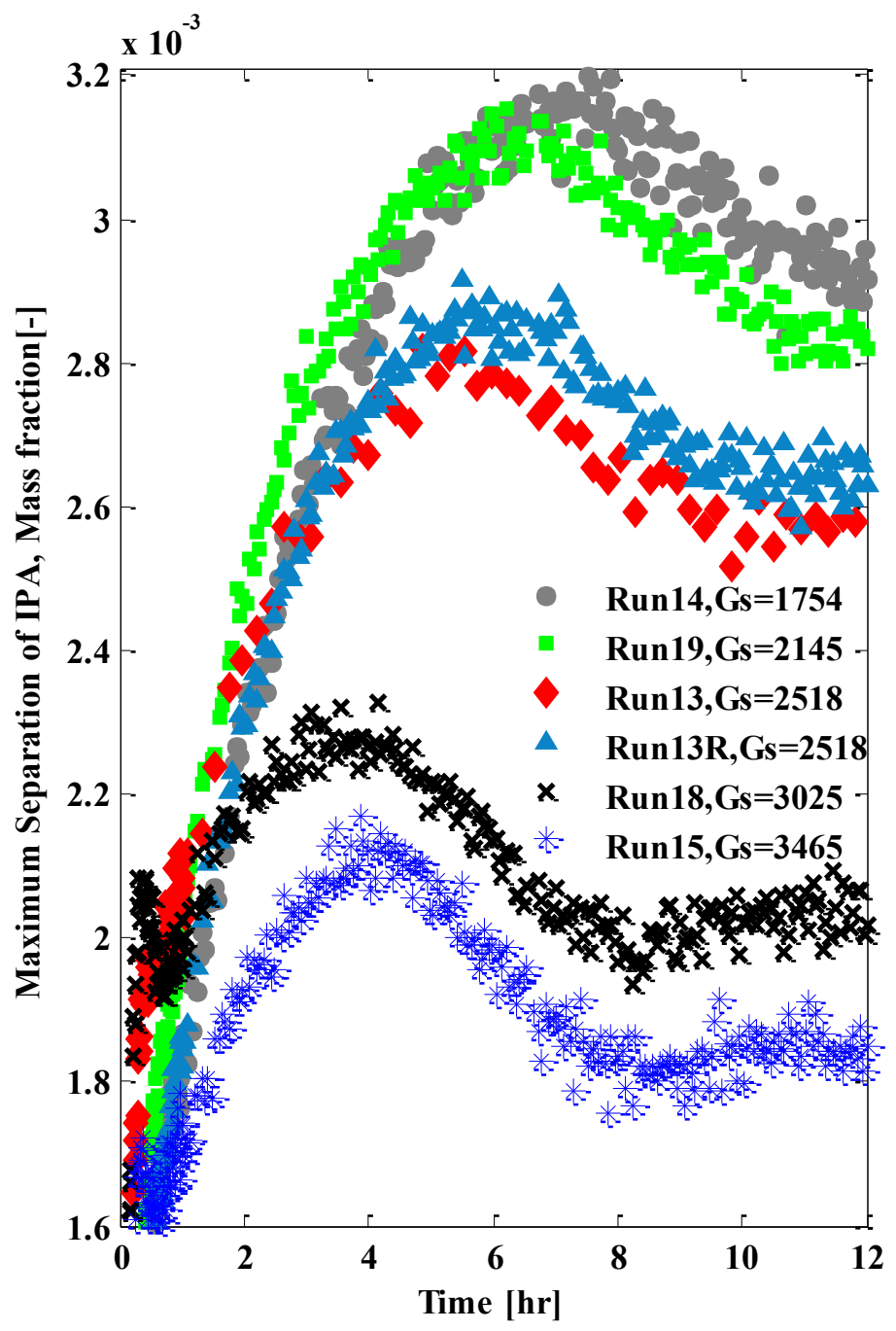

Figure 6-7 : Maximum separation of the various runs with the expanded y-axis and contracted $x-$ axis during thermodiffusion time

It indicates that from zero gravity to this critical Gershuni number the maximum separation occurred at the diffusion time. This shows that diffusion plays the dominant role in component separation at low Gershuni numbers.

Note, the $\mathrm{x}$-axes in Figure 6-8 are a calculated Gershuni number based on the temperature difference of the Peltier elements $(15 \mathrm{~K}$, lower axis) and optical temperature difference (upper 
axis). We believe that the upper axis must be used in this kind of study because the accurate temperature difference between the hot and cold walls can be measured optically. It must be noted that calculations were done with the vibrational acceleration $\left(\mathrm{g}_{\mathrm{os}}=\mathrm{A} \omega^{2}\right.$ where A represents the vibration amplitude and $\omega$ frequency of the force vibration) to investigate the possible relation of the acceleration of the vibration to the separation in the domain. While generally as a decrease in the acceleration increases the separation, no firm pattern, or relation can be found between acceleration and the separation in the cell. Thus, it can be concluded that the Gs number provides better performance to predict the effect of vibration on the mixing and induced velocity in the system.

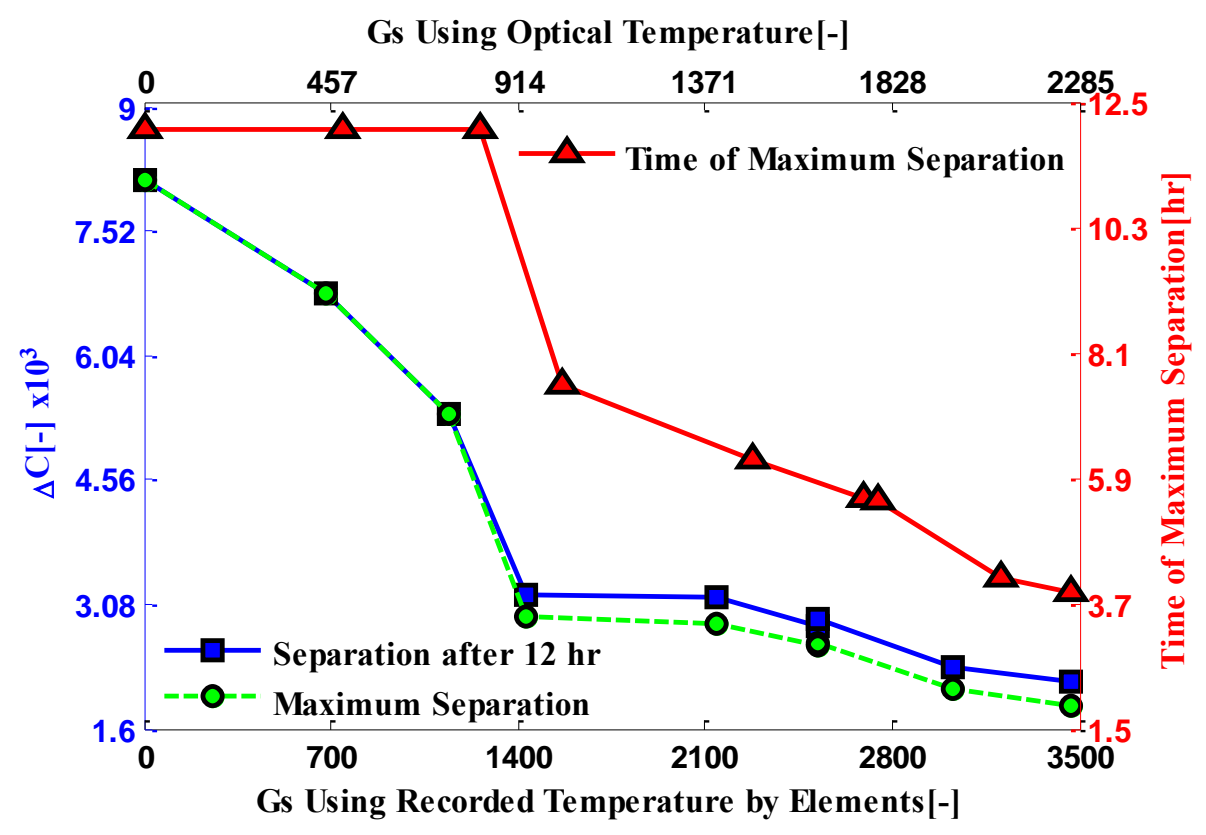

Figure 6-8 Maximum separation and the time of maximum separation versus Gershuni number for all runs.

As shown, the influence of vibration is not negligible for any run studied herein because the Gs is greater than 20. According to Gershuni method and theory, when Gs 20, 95\% of the separation of a zero gravity environment is achieved. Further increase in the Gershuni number seriously affects the diffusion process as induced convection dominates the diffusion process. However, it was found that when the Gershuni number was below 10, the process was completely governed by pure thermodiffusion. Further increase in the Gershuni number triggers mixing effects $[114,136,137]$. 


\subsection{Conclusions}

In order to determine the impact of controlled forced vibration on flow patterns, eight runs with a maximum temperature difference of $15 \mathrm{~K}$ and a negative Soret coefficient were selected and analyzed using Mach-Zehnder interferometer for the first time. Mathematical analysis by means of a Fourier transform image processing was performed. Although theoretically and according to the records of the Peltier elements, the temperature gradient was $15 \mathrm{~K}$; considering the cropped size of the images, the maximum measured gradient across the cell was less than $13.5 \mathrm{~K}$. Consequently, substantial deviation from ideal thermodiffusion resulted by considering the separation at the end of the runs due to the temperature difference recorded by the Peltier elements. In addition, using the optical result can provide an accurate measurement of Soret coefficient in this experiment as shown here.

The obtained results were compared with the analytical separation in the convection-free regime using benchmark values of diffusion coefficients from literature. Results revealed a maximum separation for the case with the minimum Gershuni number; however, a linear relationship between Gs and the maximum separation was not detected for the entire range. For Gs higher than a critical value, the effect of an increase in the vibration level decreased, and a linear decrement in separation was detected. The final results of this research are in good agreement with pervious numerical studies. While most of the runs reached a steady-state condition before the end of diffusion time, the run with the smallest Gs required more time to reach this condition. Finally, it was shown that the ISS provides a suitable environment for this experiment, and the results of the IVIDIL experiments are consistent and can be used as a reference to validate experiments in the conditions of earth gravity. 


\section{Chapter 7: New Experimental Method to Measure Pure and Cross Diffusion Coefficients}

\subsection{Introduction}

In this chapter, a digital Mach-Zehnder interferometer (MZI) that is equipped with two lasers of different wavelengths was used to conduct high-resolution measurements of concentration profiles of a ternary mixture inside a diffusion cell. An advanced image processing method was employed to extract the phase image from fringe images $[119,138]$. According to the measured refractive index variations inside the cell, the concentration profiles of the two components were measured. There exists a well-known analytical solution for the mass transfer of species in binary mixtures, it also is known that the cross diffusion coefficients of ternary mixtures are about one order of the magnitude smaller than the pure diffusion coefficients in these systems. Because of this magnitude difference, in most of the diffusion measurements of ternary mixtures the cross diffusion coefficients were neglected, and the analytical solution for binary mixtures was used to determine the pure diffusion coefficients. Here, for the first time, the refractive indices inside a ternary system measured by two lasers were coupled with the analytical solution of mass transfer in the system for use in a an evolutionary algorithm (EA) known as genetic algorithm (GA) based on Fick's law were employed to simultaneously measure the pure and cross diffusion coefficients of a transparent ternary mixture. In order to validate the proposed measurement method, the experimental results of the DSC experiments on board the International Space Station (ISS) were analyzed using this technique, and the obtained results were compared with the currently used analysis techniques. Then, the proposed approach is explained in detail, which includes a mathematical description of the problem for binary and ternary mixtures, the experimental procedures, and the optimization method and in the last section provides the experimental results gained by the proposed approach.

\subsection{Proposed Approach to Measure Diffusion Coefficients}

The proposed approach can be described by four sub-sections: 1) Mathematical differential equations that demonstrate the mass transfer in the ternary mixture, 2) analytical solutions for the 
ternary system with specific initial conditions, 3) the concepts of the MZI experiment equipped with two lasers with different wavelengths for monitoring the change of refractive index in the cross section of the cell, and 4) the optimization method, which is based on an EA.

\subsubsection{Mathematical Description of the Diffusion in a Ternary Mixture}

Diffusive mass transport in a ternary mixture in an isothermal condition is described by two independent mass fluxes according to the first Fick's law [52,53]. There is no need to define the third mass flux for the last component because the final closing relation describes the third mass flux completely. Given, i =1,2, and 3, the mass fluxes are given by Equations 7-1. to 7-3:

$$
\begin{aligned}
& \vec{J}_{1}=-\rho\left(D_{11} \nabla c_{1}+D_{12} \nabla c_{2}\right) \\
& \vec{J}_{2}=-\rho\left(D_{21} \nabla c_{1}+D_{22} \nabla c_{2}\right) \\
& \sum_{i=1}^{3} \vec{J}_{i}=0
\end{aligned}
$$

Mass conservation equations, with previous equations and the assumption of a convectionless regime, allow writing the following equations that govern the time evolution of the component concentration along with the general boundary and initial conditions (the second Fick's law):

$$
\begin{aligned}
& \frac{\partial c_{1}}{\partial t}=-\rho\left(D_{11} \nabla^{2} c_{1}+D_{12} \nabla^{2} c_{2}\right), \quad c_{1}(z, 0)=\frac{\Delta c_{1,0}}{L} z+\frac{2 c_{1,0}-\Delta c_{1,0}}{2}, \quad c_{1}(z, \infty)=c_{1,0} \\
& \frac{\partial c_{2}}{\partial t}=-\rho\left(D_{21} \nabla^{2} c_{1}+D_{22} \nabla^{2} c_{2}\right), \quad c_{2}(z, 0)=\frac{\Delta c_{2,0}}{L} z+\frac{2 c_{2,0}-\Delta c_{2,0}}{2}, \quad c_{2}(z, \infty)=c_{2,0} \\
& \sum_{i=1}^{3} \frac{\partial c_{i}}{\partial t}=0
\end{aligned}
$$

\subsubsection{Analytical Solutions for Mass Transfer}

When one assumes that the cross diffusion coefficients are negligible in comparison with the pure diffusion coefficients, Equations 7-5 and 7-6 can be simplified. The simplified forms of those equations are mentioned below: 
$\frac{\partial c_{1}(z, t)}{\partial t}=-\rho D_{11} \frac{\partial^{2} c_{1}(z, t)}{\partial z^{2}}$

$\frac{\partial c_{2}(z, t)}{\partial t}=-\rho D_{22} \frac{\partial^{2} c_{2}(z, t)}{\partial z^{2}}$

where $c_{i, 0}$ is the initial mass fraction of the $i^{\text {th }}$ component, $\Delta c_{i, 0}$ represents the initial concentration gradient of the $\mathrm{i}^{\text {th }}$ component, and $\mathrm{L}$ is the distance between the points that define the boundaries to $\Delta \mathrm{c}_{\mathrm{i}, 0}$ at the beginning of diffusion process (or the length of the cell in this experimental method). The temporal concentration variation in the system can be expressed analytically as a function of diffusion time:

$\Delta C_{1}(z, t)=C_{0,1}-\Delta C_{0,1}\left[\frac{4}{\pi^{2}} \sum_{n, o d d}^{\infty} \frac{1}{n^{2}} \cos \left(\frac{n \pi z}{L}\right) \exp \left(-n^{2} \frac{t}{\tau_{D_{11}}}\right)\right]$
$\Delta C_{2}(z, t)=C_{0,2}-\Delta C_{0,2}\left[\frac{4}{\pi^{2}} \sum_{n, o d d}^{\infty} \frac{1}{n^{2}} \cos \left(\frac{n \pi z}{L}\right) \exp \left(-n^{2} \frac{t}{\tau_{D_{22}}}\right)\right]$
$\sum_{i=1}^{3} C_{i}=1$

where $\tau_{D_{11}}=\frac{L^{2}}{\pi^{2} D_{11}}, \tau_{D_{22}}=\frac{L^{2}}{\pi^{2} D_{22}}$ that demonstrate the diffusion times for components one and two, respectively, in the ternary mixture. In previous measurements of diffusion coefficients, either for binary or ternary mixtures, Equations 7-9 and 7-10 were used to estimate the pure diffusion coefficients of the mixture. Consequently, this method was not able to measure the cross diffusion coefficients of ternary mixtures. However, in this study we did not ignore the cross diffusion coefficients, since it has been shown that in some cases the magnitudes of the cross diffusion coefficients are comparable to those of the pure diffusion coefficients. In these cases, neglecting the cross diffusion coefficients is not an accurate assumption. Consequently, in order to develop a general method to measure diffusion coefficients of ternary mixtures, all diffusion coefficients must be considered; therefore, Equations 7-5 and 7-6 should be solved with no simplification. 
Equations 7-5 and 7-6 can be rewritten as follows for the diffusion of any ternary mixture:

$\frac{\partial}{\partial t}\left[\begin{array}{l}\rho c_{1} \\ \rho c_{2}\end{array}\right]=\left[\begin{array}{ll}D_{11} & D_{12} \\ D_{21} & D_{22}\end{array}\right] \frac{\partial}{\partial z}\left(\rho \frac{\partial}{\partial z}\left[\begin{array}{l}c_{1} \\ c_{2}\end{array}\right]\right)$

The only possible method to solve this system of differential equations is by decoupling the two equations and then applying the results of binary mixture to the decoupled equations. A similar method has been used before for the same equations but with different initial conditions $[9,139,140]$; however, for the specified initial conditions mentioned in Equations 7-5 and 7-6, this the first time that the analytical solution has been derived. The diffusion matrix D can be diagonalized as:

$D=P E P^{-1}$

In which E represents the eigenvalue matrix and the matrix $\mathrm{P}$ is a function of the eigenvector

$$
\begin{aligned}
& E=\left[\begin{array}{ll}
\lambda_{1} & 0 \\
0 & \lambda_{2}
\end{array}\right] \\
& P=\left[\begin{array}{cc}
1 & \frac{D_{12}}{\lambda_{2}-D_{11}} \\
\frac{\lambda_{1}-D_{11}}{D_{12}} & 1
\end{array}\right]=\left[\begin{array}{cc}
1 & \frac{\lambda_{2}-D_{22}}{D_{21}} \\
\frac{D_{21}}{\lambda_{1}-D_{22}} & 1
\end{array}\right] \\
& P^{-1}=\frac{\lambda_{2}-D_{11}}{\lambda_{2}-\lambda_{1}}\left[\begin{array}{cc}
1 & \frac{D_{12}}{D_{11}-\lambda_{2}} \\
\frac{D_{11}-\lambda_{1}}{D_{12}} & 1
\end{array}\right]=\frac{\lambda_{2}-D_{11}}{\lambda_{2}-\lambda_{1}}\left[\begin{array}{cc}
1 & \frac{D_{22}-\lambda_{2}}{D_{21}} \\
\frac{D_{21}}{D_{22}-\lambda_{1}} & 1
\end{array}\right] \\
& \lambda_{1}=\frac{D_{11}+D_{22}+\sqrt{\left(D_{11}-D_{22}\right)^{2}+4 D_{12} D_{21}}}{2} \\
& \lambda_{2}=\frac{D_{11}+D_{22}-\sqrt{\left(D_{11}-D_{22}\right)^{2}+4 D_{12} D_{21}}}{2}
\end{aligned}
$$

Consequently, the analytical solution has the same form as the general solution of the binary as

$$
\Delta \phi_{1}(z, t)=\phi_{0,1}-\Delta \phi_{0,1}\left[\frac{4}{\pi^{2}} \sum_{n, \text { odd }}^{\infty} \frac{1}{n^{2}} \cos \left(\frac{n \pi z}{L}\right) \exp \left(-n^{2} \frac{\pi^{2} \lambda_{1} t}{L^{2}}\right)\right]
$$


$\Delta \phi_{2}(z, t)=\phi_{0,2}-\Delta \phi_{0,2}\left[\frac{4}{\pi^{2}} \sum_{n, \text { odd }}^{\infty} \frac{1}{n^{2}} \cos \left(\frac{n \pi z}{L}\right) \exp \left(-n^{2} \frac{\pi^{2} \lambda_{2} t}{L^{2}}\right)\right]$

where $\vec{\phi}_{0}=P^{-1} \vec{c}_{0}$ and $\Delta \vec{\phi}_{0}=P^{-1} \Delta \vec{c}_{0}$, and $\lambda_{i}$ and $\mathrm{P}$, are as defined above [140]. Since the solution for ternary mixtures is a general solution for mass transfer due to diffusion, mass transfer in a binary mixture can also be expressed by these formulas when $D_{11}=D_{22}$ and $D_{12}=D_{21}=0$. It must be noted that a numerical discretized form of Equations 7-19 and 7-20 can be solved numerically versus time for various estimated parameters using an iterative method rather than an analytical solution. However, the accuracy of the numerical solution must be validated versus the analytical solution of the ternary mixture.

\subsection{Application of MZI to Visualize Mass Transfer}

Equation 7-21 can be used to define the relationship between the refractive index and concentration change for binary mixtures.

$$
\Delta n(x, y)=\left(\frac{\partial n}{\partial c_{1}}\right)_{T_{0}, P, \lambda_{1}} \Delta C_{1}(x, y)
$$

Thus, if the refractive index variation respect to the homogeneous condition can be measured, the concentration profile of one of the components can be calculated. It must be noted that Equation 7-21 is only applicable when the concentration change is caused due to change of concentration alone. In other words, there should not be any local temperature gradient or change of the temperature with respect to time. For the ternary system, there are two sources of change of refractive index caused by two components, as:

$$
\Delta n_{1}(x, y)=\left(\frac{\partial n_{1}}{\partial c_{1}}\right)_{T_{0}, C_{2}, \lambda_{1}} \Delta C_{1}(x, y)+\left(\frac{\partial n_{1}}{\partial c_{2}}\right)_{T_{0}, C_{1}, \lambda_{1}} \Delta C_{2}(x, y)
$$

There are two contrast factors in the above equation that relate to the concentration change of components one and two. The refractive index $(\Delta n)$ from one source (one laser) for a ternary system cannot provide enough information to determine both $\Delta \mathrm{c}_{1}$ and $\Delta \mathrm{c}_{2}$. On the other hand, the refractive index of a material varies with the wavelength (and frequency) of light. As a result of 
this, if another laser with a different wavelength is used (simultaneously along with the first laser), two equations would result that makes the measurement of $\Delta \mathrm{c}_{1}$ and $\Delta \mathrm{c}_{2}$ possible.

$$
\Delta n_{2}(x, y)=\left(\frac{\partial n_{2}}{\partial c_{1}}\right)_{T_{0}, C_{2}, \lambda_{2}} \Delta C_{1}(x, y)+\left(\frac{\partial n_{2}}{\partial c_{2}}\right)_{T_{0}, C_{1}, \lambda_{2}} \Delta C_{2}(x, y)
$$

It must be noted that the contrast factors are functions of both composition and temperature [141-143]; however, they can be assumed constant when the change of the concentration or temperature is small.

\subsubsection{Experimental Procedures}

The first step to making a measurement was the thermalization of the sample at the mean temperature to initialize the mixture characteristics by reaching uniform concentration and temperature distributions inside the cavity. Then, the temperature gradient was established over the next 6 to 8 hours. Because of the Soret effect, the linear concentration variations between the walls were also created by the presence of the thermal gradient[110,112,144,145].

After the completion of this step, the temperature difference was removed, and the cell was brought back to the mean temperature within three minutes. The data for the active cell were then acquired for 4 to 6 hours. After each run, the system was brought to the initial isothermal and homogeneous concentration conditions. Finally, images are analyzed by using one of the phase extraction methods like Fourier transform.

\subsubsection{Advanced Image Processing Aided with an Evolutionary Algorithm}

A sample of the fringe pattern obtained by the interferometer on board the ISS, its phase map, and the unwrapped images are shown in Figure 7-1. The arrows inside the fringe pattern (Figure 7-1a) point out various sources of noises in the image processing procedure that were caused by the optical system. By using a proper image processing, phase extraction, and an advanced unwrapping method, the negative impacts of this noise can be removed from the profile. As is shown in Figure 7-1b and c, the noise effects are completely removed from the phase and the unwrapped image $[119,146]$. 


$$
\phi_{\text {continous }}\left(x_{j}, y_{i}\right)=\phi\left(x_{j}, y_{i}\right)+2 \pi n\left(x_{j}, y_{i}\right), \quad i, j=1,2, \ldots
$$

In general, a smooth two-dimensional profile of phase change can be obtained over the entire domain using equation 7-24 in which $\mathrm{n}$ can be obtained from Equations 7-25 to 7-27. The first equation gives the phase unwrapping in one dimension, and the second one expands it along the second dimension. Basically, during this procedure, the wrapped phase of a pixel was compared with a neighboring pixel that was previously validated. If the difference between two neighbor pixels was less than $\pi, n$ remained unchanged. The phase was changed to $2 \pi$ plus its wrapped phase if the difference was less than $-\pi$. Finally, if the difference between the two pixels was more than $\pi$, the phase was equal to its wrapped phase minus $2 \pi$. It must be noted that unlike most other unwrapping techniques, the unwrapping process started from the pixel that was located at the center of the image, moving to both edges to avoid expanding any effects of noise close to the boundaries.

$n\left(x_{m / 2}, y_{n / 2}\right)=0$

$n\left(x_{m / 2}, y_{i}\right)= \begin{cases}n\left(x_{m / 2}, y_{i-1}\right) & \text { if }\left|\phi\left(x_{m / 2}, y_{i}\right)-\phi\left(x_{m / 2}, y_{i-1}\right)\right|<\pi \\ n\left(x_{m / 2}, y_{i-1}\right)+1 & \text { if } \phi\left(x_{m / 2}, y_{i}\right)-\phi\left(x_{m / 2}, y_{i-1}\right) \leq-\pi \\ n\left(x_{m / 2}, y_{i-1}\right)-1 & \text { if } \phi\left(x_{m / 2}, y_{i}\right)-\phi\left(x_{m / 2}, y_{i-1}\right) \geq \pi\end{cases}$

where $j=\frac{m}{2}, \frac{m+2}{2}, \frac{m+4}{2}, \ldots, m$ and $j=\frac{m}{2}, \frac{m-2}{2}, \frac{m-4}{2}, \ldots, 1$.

$$
n\left(x_{j}, y_{i}\right)= \begin{cases}n\left(x_{j-1}, y_{i}\right) & \text { if }\left|\phi\left(x_{j}, y_{i}\right)-\phi\left(x_{j-1}, y_{i}\right)\right|<\pi \\ n\left(x_{j-1}, y_{i}\right)+1 & \text { if } \phi\left(x_{j}, y_{i}\right)-\phi\left(x_{j-1}, y_{i}\right) \leq-\pi \\ n\left(x_{j-1}, y_{i}\right)-1 & \text { if } \phi\left(x_{j}, y_{i}\right)-\phi\left(x_{j-1}, y_{i}\right) \geq \pi\end{cases}
$$

where $j=\frac{n}{2}, \frac{n+2}{2}, \frac{n+4}{2}, \ldots, n$ and $j=\frac{n}{2}, \frac{n-2}{2}, \frac{n-4}{2}, \ldots, 1$ and also where $\mathrm{i}$ and $\mathrm{j}$ represent different coordinate indices of pixels in the $\mathrm{x}$ and $\mathrm{y}$ directions, respectively, and $\mathrm{m}$ and $\mathrm{n}$ are the number of pixels in the $\mathrm{x}$ and $\mathrm{y}$ directions, respectively [112,113,119]. 


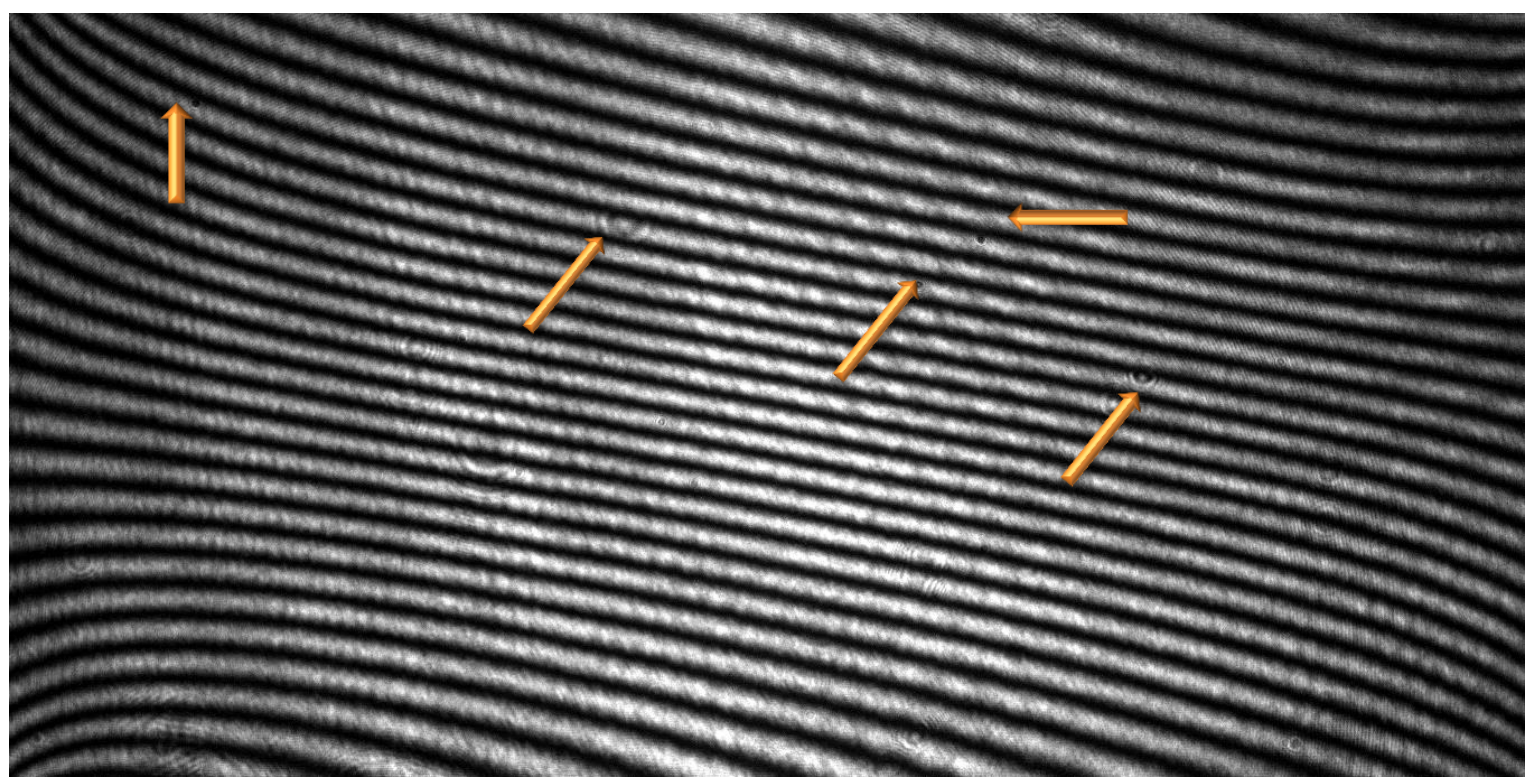

(a) A sample of fringe pattern

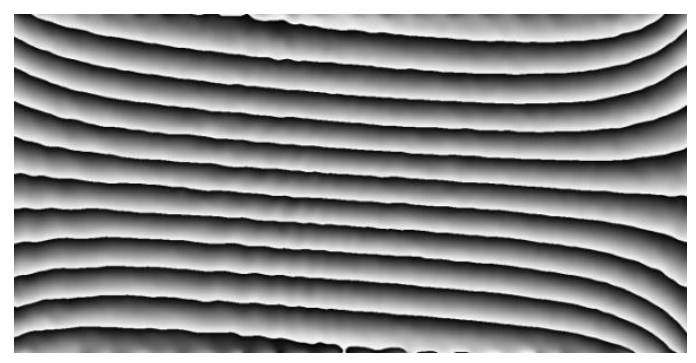

(b) its phase image

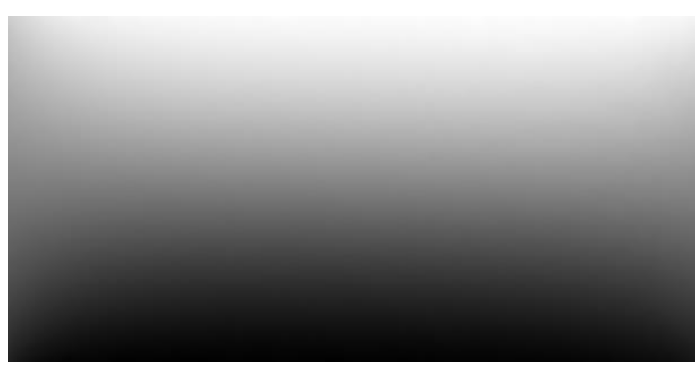

(c) unwrapped phase image

Figure 7-1: Filteration of noise in the fringe pattern by image processing

The phase differences obtained by the described unwrapping process for both of the lasers were used to measure refractive index profiles, $\Delta \mathrm{n}(\mathrm{x}, \mathrm{y})$. The profiles then allowed the measurement of the concentration profiles in the cell, and as a result the maximum $\Delta \mathrm{C}$ in the cavity. The relationship between phase difference $\Delta \phi$ and $\Delta \mathrm{n}$ is described below:

$\Delta n_{i}(x, y)=n_{i}(x, y)-n_{i, r e f}(x, y)=\frac{\lambda_{i}}{2 \pi L} \Delta \phi_{i}(x, y)$

A general sample of the change of refractive index between the walls during the entire experiment is plotted in Figure 7-2. At first, there is no change of refractive index. Then, there is a sudden jump in the refractive index caused by the application of the temperature gradient. 
Gradually, the concentration gradient built up between the walls. Subsequently, the temperature gradient was removed and in the last step, the pure diffusion process occurs.

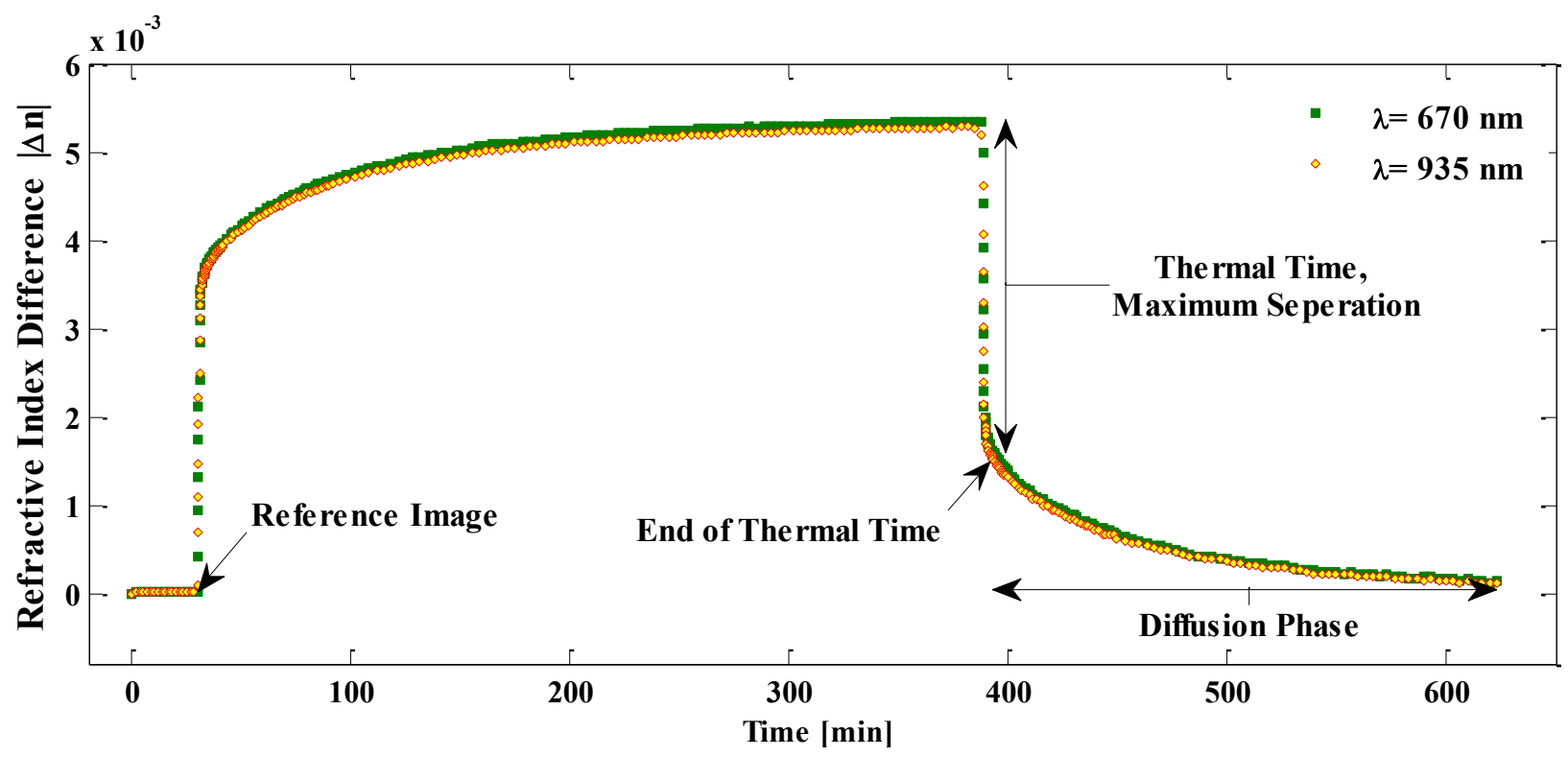

Figure 7-2: Maximum refractive index difference between the hot and cold walls during the entire duration of the MZI experiment.

The concentration profiles can then be calculated combining Equations 7-22 and 7-23, when the refractive index profiles and the concentration contrast factors of the mixture are known. The maximum refractive index variations measured by both lasers during the diffusion phase for the test mixture in this study is illustrated in Figure 7-3 (more detail on the experimental results are provided in the next section).

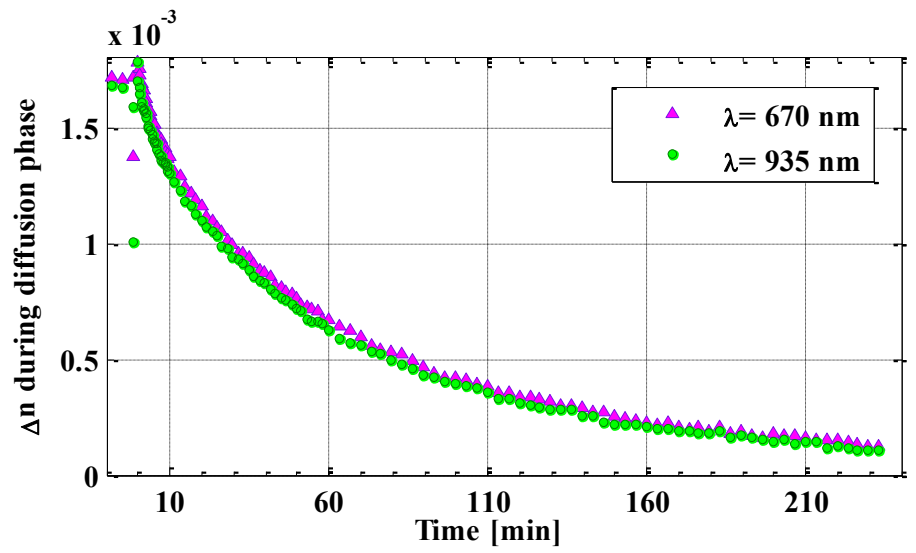

Figure 7-3: Maximum refractive index variations of both lasers with different wavelengths during the diffusion phase. 
So, the mass fraction of the first two components can be calculated as follows:

$\left[\begin{array}{l}\Delta C_{1}(x, y) \\ \Delta C_{2}(x, y)\end{array}\right]=\left[\begin{array}{ll}\frac{\partial n_{1}}{\partial c_{1}} & \frac{\partial n_{1}}{\partial c_{2}} \\ \frac{\partial n_{2}}{\partial c_{1}} & \frac{\partial n_{2}}{\partial c_{2}}\end{array}\right]_{T_{0}, P_{0}}^{-1}\left[\begin{array}{l}\Delta n_{1}(x, y) \\ \Delta n_{2}(x, y)\end{array}\right]$

When the separation profiles of two of the components are measured, the third profile can be found based on the conservation of mass in the system. Thus, the temporal concentration profile of each mixture was obtained. These profiles were used to extract the diffusion matrix. The aided genetic algorithm (GA) with fast robust discretized smoothing was implemented to find the curve that provided the best fit to the experimental concentration difference.

\subsubsection{Genetic algorithm and implementation of robust smoothing}

In any experiment, it can be shown that interpolation is unsatisfactory because it fails to account for the errors or uncertainty in the data, and the uncertainty, error, and rough data must be removed from the temporal results. We have instead opted to use data smoothing techniques, which eliminate noise and extract real trends and patterns [108,147-149]. Basically, signals are assumed to vary smoothly, perhaps with a few abrupt shifts [108].In the proposed method here, a fast robust version of a discretized smoothing spline was used $[108,150]$ based on a discrete cosine transform (DCT). The accuracy of this model has been illustrated in a few cases using MATLAB [108]. The applications of the robust smoothing DCT on the real experimental results are shown with the solid and the dashed lines in Figure 7-4.

After applying the robust smoothing method to the experimental data points, the analytical solution for the mass diffusion in ternary mixtures must be used to find the best fit to experimental results (or the best smooth curve of experimental results). The best fit lines can estimate the diffusion matrix, which includes four unknowns: $D_{11}, D_{22}, D_{12}$ and $D_{21}$. In the case that the number of free parameters is more than the number equations, those parameters can be evaluated and corrected during each iteration using the curve-fitting problem with an EA approach. In this case, for any pair of images taken by the lasers during the diffusion experiment, there were two equations with four free parameters that needed to be evaluated. Moreover, there 
were at least four constraints to be considered in order to find an optimal solution that satisfies the physics of the problem.

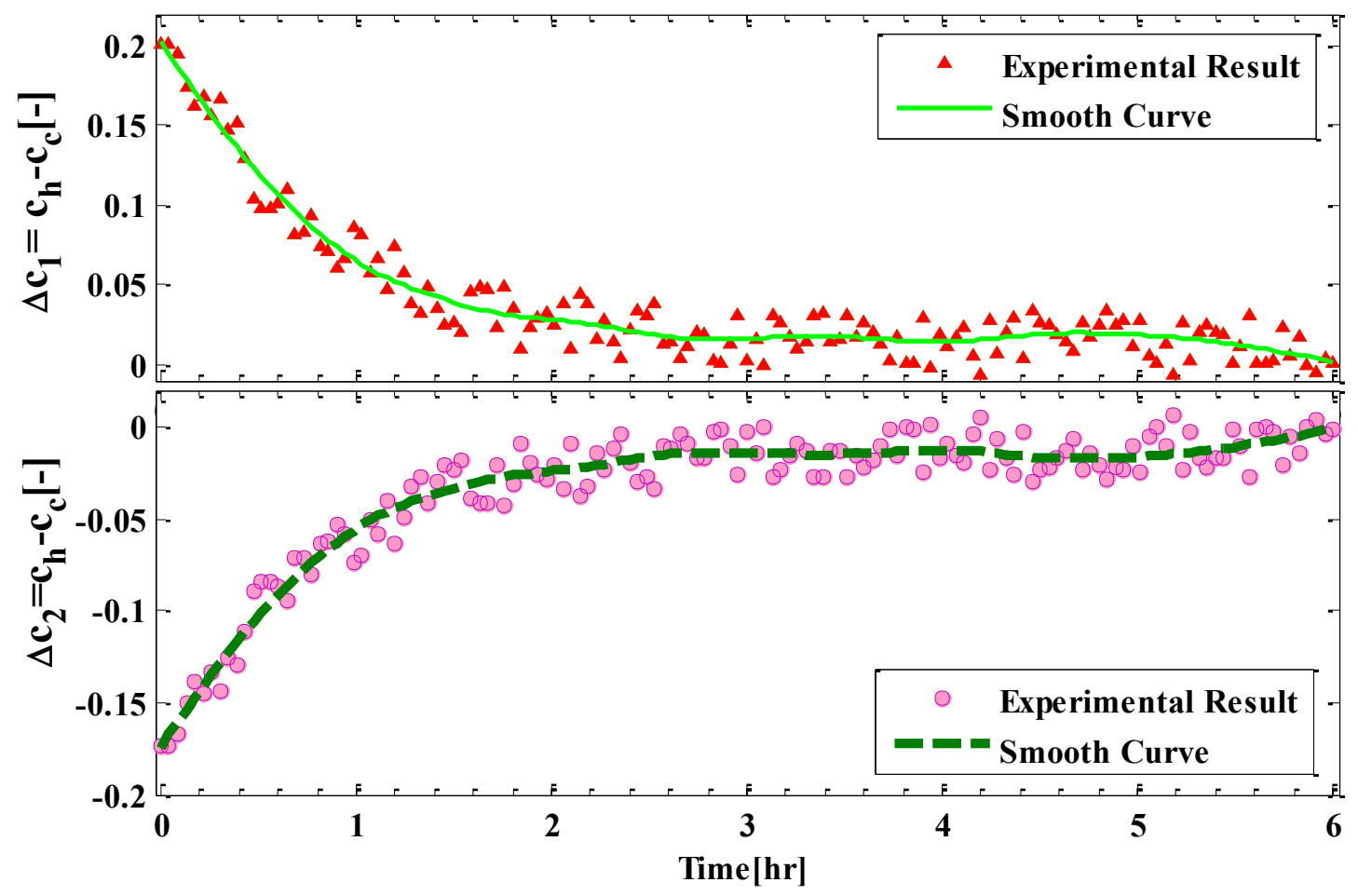

Figure 7-4: Application of robust smoothing discrete cosine transform on diffusion experiment. These constraints were introduced in the literature [140] as:

$D_{11}>0$

$D_{22}>0$

$D_{11} D_{22}-D_{12} D_{21}>0$

$\left(D_{11}-D_{22}\right)^{2}+4 D_{12} D_{21} \geq 0$

In addition to the constraints mentioned above, finding the optimal solution in the 4-dimensional domain using a gradient base method has been shown to be impossible[151]. Evolutionary 
methods (such as GA) are faster and more accurate than gradient-based methods for such this problem, because these methods do not become trapped in local optimal points, and they can consistently report the global solution. Therefore, the application of a GA for such nonlinear problems is highly recommended. The objective function must be defined in a way to minimize the difference between the predicted analytical curve and the experimental results (or smoothed experimental curve). Moreover, as time passed during the experiment, the concentration difference between the walls decreased, because of the diffusion process. When the concentration variation was small the influence of the noise and errors were more pronounced. Since at the end of the diffusion process (last hour of the diffusion process) the $\Delta \mathrm{C}$ between the walls was very small (less than $10^{-4}$ ), the influence of noise caused fluctuations in the curve of separation versus time. These fluctuations can cause noticeable variation between the experimental and fitted curves; variation that increases the objective function and has a negative impact on the final optimum solution. Thus, the objective function must be a function of time in the form of a least square error function, and it must be minimized during the optimization procedure. The suggested form of the objective function is introduced here as:

Err $=\sum_{i=1}^{n}\left[\frac{1}{n}\left(\tau_{D}-t\right)\left(C_{\text {exp-smooth }}-C_{\text {fit-cure }}\right)^{2}\right]$

where $\mathrm{n}$ is the number of images obtained by each of the lasers, and $\mathrm{t}$ is the time used to intensify the influence of each data point. Use of the the diffusion time $\tau_{D}$ in an objective function caused a linear variation between the first and the last data points in a way that the effect on the objective function of the first image was two times larger than the effect of the last one.

The results of the optimization procedure satisfied the constraints of the problem, which are mentioned in Equations 7-30 to 7-33. The first two constraints were automatically be satisfied when the lower search band of the pure diffusion coefficients were assigned zero. However, the last two constraints were applied to the optimization procedure using a penalty method. In the case that all constraints in Equation 7-30 to 7-33 were not satisfied, the objective function in Equation 7-34 was increased by a factor of three plus a constant positive value. Doing this assured that the optimized solution satisfied the constraints. The specific parameters of the 
genetic algorithm that were recommended for the estimation of the pure and the cross diffusion coefficients are provided in Table 14. These bands, limitations, and populations may change from case to case depending on the properties of the mixture and the experimental conditions.

Table 14: Genetic Algorithm Specification

\begin{tabular}{|c|c|c|c|c|c|}
\hline & & \multicolumn{2}{|c|}{$\begin{array}{c}\text { Estimation of Pure diffusion } \\
\text { coefficient }\end{array}$} & \multicolumn{2}{c|}{$\begin{array}{c}\text { Estimation of cross diffusion } \\
\text { coefficient }\end{array}$} \\
\hline Iteration & Population & Lower band & Upper band & Lower band & Upper band \\
\hline 300 & 500 & 0 & $1 \times 10^{-9}$ & $-5 \times 10^{-10}$ & $5 \times 10^{-10}$ \\
\hline
\end{tabular}

\subsubsection{Applications of Proposed Method for Space Experiments}

A mixture of tetrahydronaphthalene-isobutylbenzene-dodecane (THN 45\%[wt], IBB 10\%[wt], $\mathrm{C}_{12} 45 \%[\mathrm{wt}]$ ) was hosted on board the ISS. The liquid mixture was contained in a $10 \mathrm{~mm} \times 10$ $\mathrm{mm} \times 5 \mathrm{~mm}(\mathrm{w}, 1, \mathrm{~h})$ cell and was monitored by a MZI setup. The experiment was performed at a mean temperature of $298 \mathrm{~K}$. The optical properties of this ternary mixture at this specific composition can be found in literature [95].

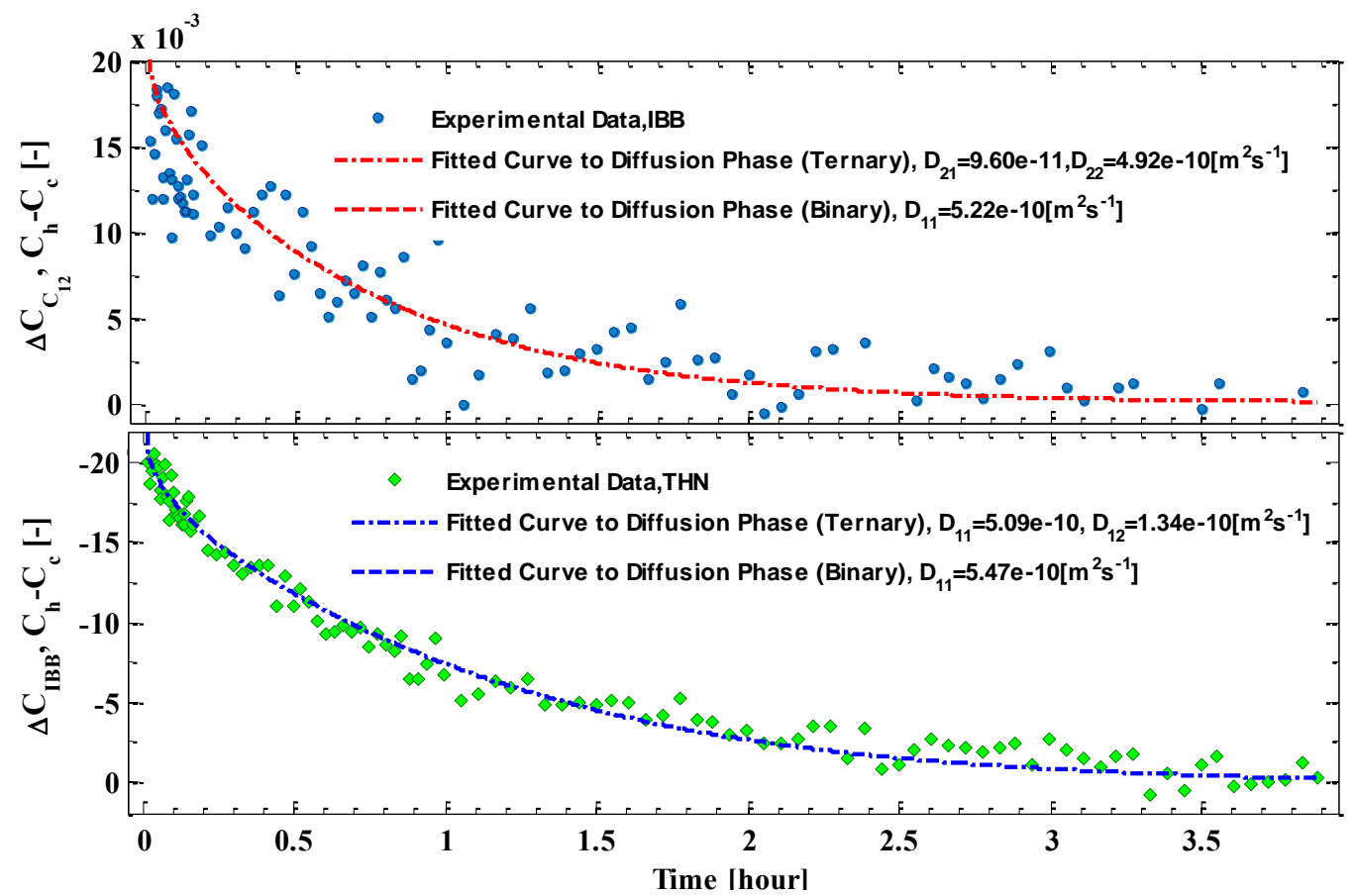

Figure 7-5: Temporal concentration variation between the walls during the space experiment and the fitted curves obtained based on the analytical solution of the binary and ternary mixtures.

The maximum concentration difference, $\Delta \mathrm{C}$, for the test mixture was extracted from the chart shown in Figure 7-3. The calculated concentration variations between walls are illustrated in Figure 7-5 for $\mathrm{C}_{12}$ (at the top) and IBB (at the bottom). The smooth patterns of concentration 
differences of the components (especially for IBB) can be observed in Figure 7-5. The separation magnitudes of both $\mathrm{C}_{12}$ and IBB were similar, which means that for this case the measurement of the diffusion coefficient of THN is not easily made, because the maximum concentration gradient of THN at the beginning of the diffusion experiment is not noticeable.

The GA was applied to the obtained concentration variations for the diffusion phase of the experiment, which lasted 4 hours. In addition to this procedure, the analytical solution for the binary mixture was used to find the best fit to the experimental results. Interestingly, both fitted curves (one obtained by the analytical solution of the ternary mixture and the other one from the binary mixtures) overlapped each other completely (see Figure 7-5). This shows that both procedures provide the same accuracy to estimate the best curve to fit the experimental results. However, the cross diffusion coefficients in the binary mixture case were ignored. If one compares the values of $\mathrm{D}_{11}$ obtained by both (see Figure 7-5), then the small difference between these two pure diffusion coefficients is observed. It must be added that the pure diffusion coefficient estimated by the proposed method in this study has a smaller value than that estimated by the other method. This difference can be explained by considering the cross diffusion coefficient that is also estimated by this technique. When the analytical solution of the binary mixture is being used instead of the ternary one, pure diffusion represents the summation of the effects of both the pure and cross diffusion coefficients. Using the proposed technique, on the other hand, distinguishes the impact of these two diffusion coefficients and reports them as independent values.

\subsubsection{Advantages and Limitations of the Proposed Method}

The proposed method is applicable to most of the ternary mixtures and has an important advantage that other methods do not have. To measure the diffusion coefficients, there must be a local concentration gradient for at least one of the components of the mixture at the beginning of the procedure $(\mathrm{t}=0)$. Most experimental methods propose two mixtures containing similar components but different initial concentrations of the components in contact of each other at the beginning of the experiment. Proposing these two mixtures at $t=0$ introduces many technical difficulties to the experimentalists, and it also requires direct contact between the fluid media. This connection can disturb the pure diffusion procedure and may have an influence on the 
measurement of the diffusion coefficient. On the other hand, in the method proposed here, the Soret effect is used as the driving force to induce a linear variation of the concentration gradient inside the domain. This method does not need any contact with the medium and also provides a linear variation of the concentration as the initial condition of the problem.

Despite this benefit provided by the Soret effect, using this phenomenon to create the concentration gradient has its limitations. In order to have a large enough concentration gradient $\left(\sim 8 \times 10^{-3}[\mathrm{wt}]\right)$ to enable clear measurements and detection of the diffusion process, the liquid mixture should have a noticeable tendency to separate in the presence of a thermal gradient. In other words, this technique does not work for mixtures with small Soret coefficients $\left(<10^{-3}\right)$. This also depends on the other characteristics of the experiment, such as $\Delta \mathrm{T}$, the initial concentration gradient, and the cell size.

One may suggest applying a higher temperature gradient for the cases with low values of Soret coefficient in order to compensate for this limitation. In the presence of higher temperature differences, higher concentration variations will be caused for any binary or ternary mixture. However, there are two points that must be considered. First, there is a constraint to increases in $\Delta \mathrm{T}$. The maximum temperature difference applied to the system must be in a region that the contrast factors can be assumed constant. Since the contrast factors are a weak function of temperature, the maximum value for $\Delta \mathrm{T}$ has an approximate upper band at $20 \mathrm{~K}$. The second limitation is only true for ternary mixtures and can be explained as follows. If the separation of one of the components in comparison with the two others is small in the ternary mixture, it is not possible to measure the diffusion coefficients for that component. However, it must be noted that even in this case the diffusion coefficients of the two other components can be easily measured using this method.

The quality of the images, the number of fringes, the angle of mirror, and the length of the path must all be optimized in the MZI setup to obtain an accurate and precise result. The contrast factor matrix in Equation 7-29 is the key point to relate the refractive index variation to the concentration. Since the concentration contrast factors of a specific mixture at various wavelengths are remarkably close, and also $\Delta \mathrm{n}$ measured from the experiment on board the ISS by both lasers are quite similar (see Figure 7-3 that shows the similar behavior of both lasers for 
the test mixture), a small change in the contrast factor will change the final result. It should be emphasized that this is a general limitation of MZI to measure the concentration profiles for ternary mixtures. In order to minimize this effect, two lasers that are used in the experiment must have the maximum possible wavelength difference. For instance instead of using lasers with $\lambda=$ 670 and 935 [nm], it is recommended to use two lasers that have $\lambda=450$ and 935 [nm].

\subsection{Conclusions}

A new experimental method to measure the pure and cross diffusion coefficients of any transparent liquid mixture using Mach-Zehnder interferometry has been proposed. The essential post-processing procedure that was required to extract the diffusion matrix from the fringe images is provided in detail. The Soret phenomenon was used as the driving force to initiate the concentration variation at the beginning of the diffusion process. The analytical solution of the diffusion mass transfer in a ternary system along with an optimization method using an evolutionary algorithm was employed to estimate the values of the diffusion matrix. The purpose of this technique was examined versus the experimental results obtained on board the International Space Station. The comparison between the measured diffusion matrix found by the proposed method and the previous technique (using the analytical solution for a binary mixture) proves the accuracy of the proposed technique. Furthermore, this technique can in fact provide more details. Eventually, the advantages and limitations of this technique were discussed and the essential recommendations and suggestions were made to minimize the influences of the mentioned limitations. 


\section{Chapter 8: Detail Analysis of DSC Experiment to Measure the Soret coefficients for a ternary hydrocarbon mixture}

\subsection{Introduction}

The gap of experimental data for mixtures more than two components has a negative impact of the development of the multi-component thermodiffusion models $[35,50]$. Although the theory for binary mixtures is well established, there is some uncertainty related to the ternary system. Accordingly, there was an essential requirement for an accurate ternary experiment to confirm ternary or multi-component models.

While the IVIDIL experiment was proposed to study the impact of oscillatory acceleration on the thermo separation of binary mixtures [67,152-154], the main purpose of DSC (Diffusion and Soret Coefficient) was the measurement of diffusion coefficients of some ternary mixtures, in the reduced gravity environment available on board the ISS [44,132].

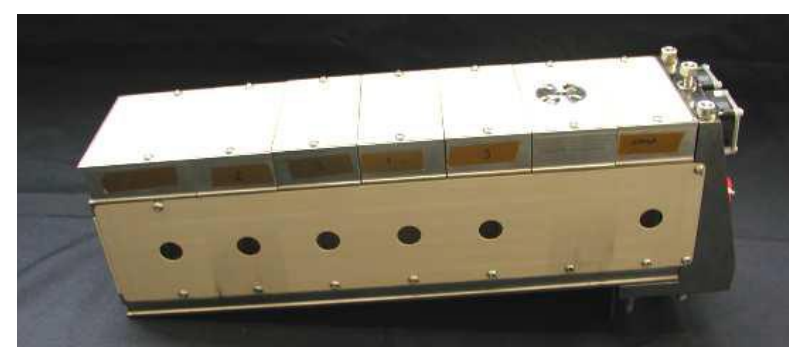

Figure 8-1: SODI cells arrays $[111,133,152]$

Accordingly, it is expected that this experiment may deliver high precision benchmark measurements on scientifically and industrially relevant multi-component mixtures. The sample was contained in a cell $10 \mathrm{~mm} \times 10 \mathrm{~mm} \times 5 \mathrm{~mm}(\mathrm{w}, 1, \mathrm{~h})$ and is monitored by a Mach-Zehnder interferometer at two wavelengths. Mixtures of tetrahydronaphthalene-isobutylbenzenedodecane (THN-IBB- $\mathrm{C}_{12}$ ) at five different compositions were hosted in the DSC cell array.

\subsection{Chapter Aims}

In this chapter, two selected DSC experiment runs, which were performed on board the International Space Station, were investigated in detail for the first time. The ternary sample was 
contained in a cell $10 \mathrm{~mm}$ x $10 \mathrm{~mm}$ x $5 \mathrm{~mm}(\mathrm{w}, 1, \mathrm{~h})$ and was monitored using a Mach-Zehnder interferometer at two wavelengths. Mixtures of THN-IBB-C $\mathrm{C}_{12}$ at five different compositions were hosted in a cell array. The experimental results of these experiments correspond to the second cell of the DSC array of the a mixture of $80 \%$ mass fraction of IBB, and $10 \%$ mass fraction of THN, and $10 \%$ mass fraction of $\mathrm{C}_{12}$ is presented in this work. Thus, Run7 and Run12 according to DSC timetable with mean temperature of $298 \mathrm{~K}$ are investigated in this work. This series of images needed to be processed and analyzed by means of different Fourier transformations or phase shifting methods. Accordingly, temperature and concentration differences in the cavity caused refractive index variations in the domain that must be obtained from this process. Then, the Soret coefficient is obtained with two different points of view: 1) by assuming the system has reached steady state and then 2) by curve fitting using the genetic algorithm. These two results prove that unlike the IVIDIL experiment for which steady-state condition was not reached at the end of the thermodiffusion phase, the DSC experiment provided enough time for this phase of experiment to reach steady state. While perfect thermal isolation near the lateral walls was not obtained, separation of the components in the mixture was measured in the middle of the cavity, a region in which the mixture behaves in the same manner as pure diffusion. Section 2 explains the optical setup and Section 3 presents the experimental procedure.

\subsection{Optical Experimental Equipment for Ternary System}

The proposed experiments in space were implemented in the SODI facility. The main feature of the SODI apparatus is located in the MSG (Microgravity Science Glovebox), shown in Figure 8-2. In the modular approach, where the experiment module is the central element, it is possible to change some parts of the SODI without any significant disruption to the diagnostic instruments or having to take out the facility from the MSG. For example, switching from one experiment to another requires only changing the experimental cells or cell arrays as mentioned earlier and shown in Figure 8-2. The mixture components have been chosen because they are representative of a hydrocarbon reservoir mixture [35]. THN represents the family of the naphthenic compounds, while IBB presents the aromatic compounds, and finally $\mathrm{C}_{12}$ provides the aliphatic compounds' behavior. The chemical structures, properties, and their molecular 
shapes are provided in Table 15. The decision for the selected fluid depended on two parameters: first, the ground-based experimental results, and the possibility of being investigated using SODI and the feasibility of data analysis.

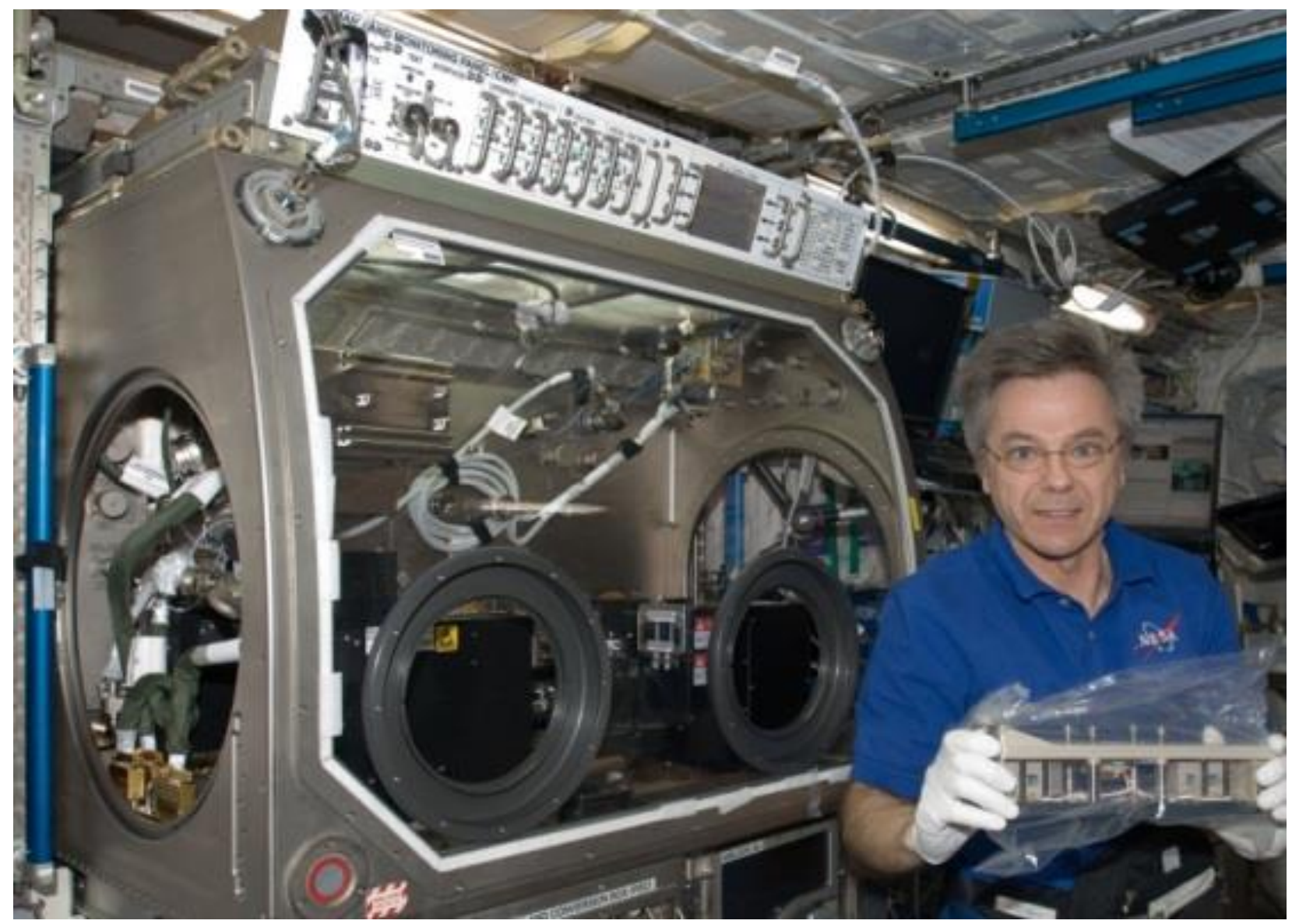

Figure 8-2: Glovebox and CSA astronaut Thirsk, onboard ISS before installing SODI array inside that box

Figure 8-2a and Figure 8-3a show the actual apparatus and schematic setup, respectively, of the MZI instrument, while Figure 8-2b and Figure 8-3b illustrate the experimental and schematic setup, respectively, of the particle image velocimetry experiment used in this experiment. The fixed optical module is used to perform velocimetry on the DSC and IVIDIL companion cells.

Five different compositions of the THN-IBB-C 12 mixture are considered in the first cell array of DSC, as mentioned in detail in Table 15, while the second cell array is filled with different compositions of mixtures of cyclohexane-toluene-methanol. The last part of Figure 8-2 and 
Figure 8-3 shows the assembled apparatus. There are two temperature controllers to adjust the temperature at the lower and upper sides of the cells.

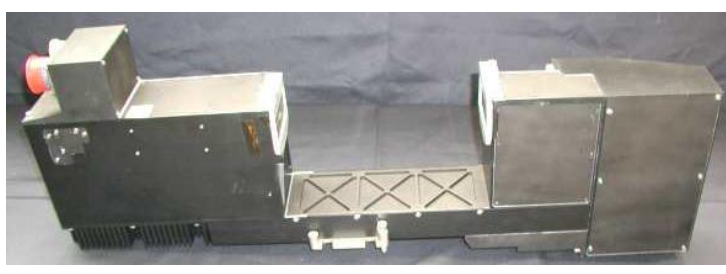

(a) Interferometer moving bridge

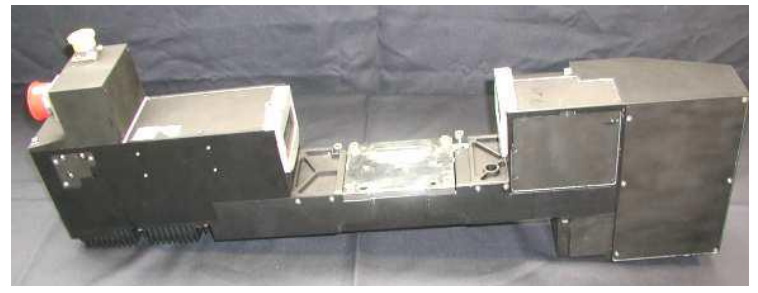

(b) Interferometer fixed bridge

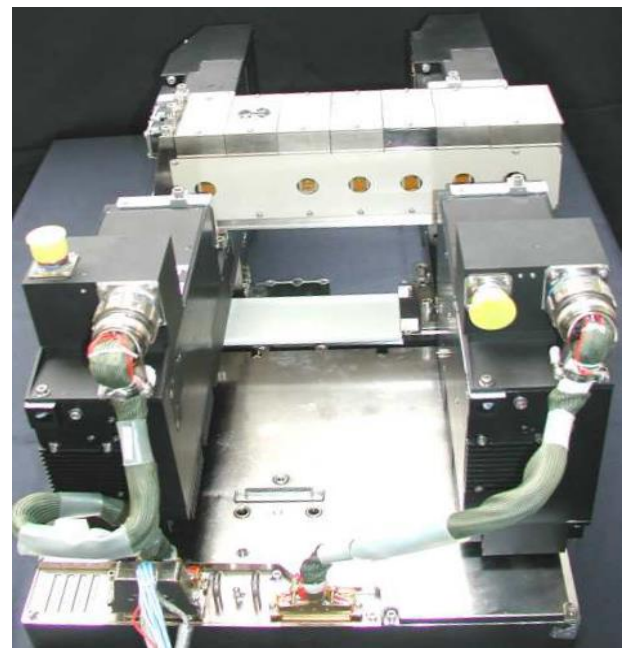

(c) Assembled SODI facility

Figure 8-3 The actual SODI apparatus $[111,155]$

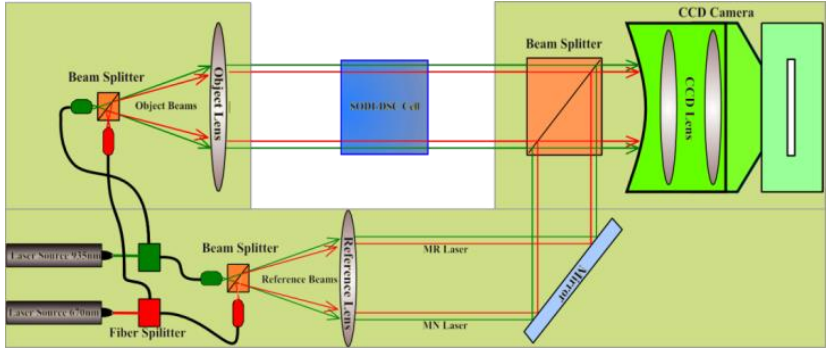

(a) Interferometer moving bridge

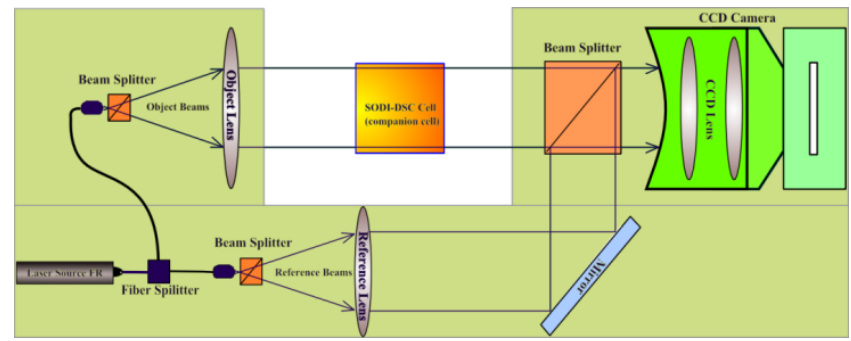

(b) Interferometer fixed bridge

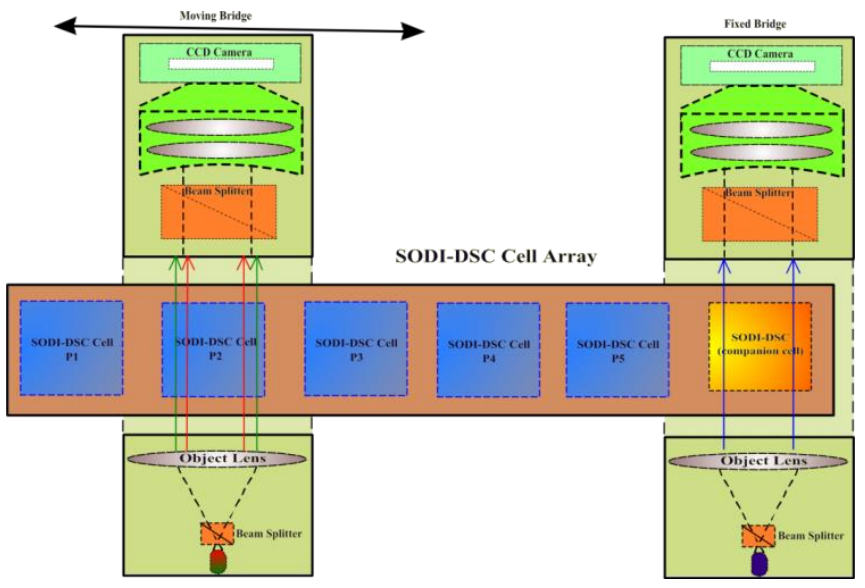

(c) Assembled SODI facility

Figure 8-4 The scheme of the SODI apparatus

These two regions are covered by two copper blocks that homogenize the temperature throughout the liquid side instantly as a result of its high thermal conductivity. The temperatures of these regions, which yield the temperature gradient for the liquid volume, are controlled. This unit includes a calibrated thermal sensor for temperature reading with a high precision value of 
$0.01 \mathrm{~K}$ as well as the Peltier element air circulation channel. In addition to these, there is a PID controller system, which sends and receives the temperature reading to a computer. An optimal PID tuner is used to propose the optimal action in the operative feedback controller loop.

Table 15: Chemical properties of three components of the test mixtures

\begin{tabular}{|c|c|c|c|}
\hline $\begin{array}{c}\text { EPA Substance Registry } \\
\text { System }\end{array}$ & \begin{tabular}{c} 
1,2,3,4-Tetrahydronaphthalene \\
\hline Abbreviation name
\end{tabular} & Isobutyl benzene & n-Dodecane \\
\hline Molecular Formula & THN & IBB \\
\hline Molecular Structure & & \\
& & \\
& & \\
\hline
\end{tabular}

The cell configuration was selected based on optimization analysis of different aspect ratios of the Soret cell, and it is made of quartz Suprasil [131]. Mialdun et al. have discussed cell size and shape optimization, and the importance of its thermal design in their recent work. The new cell is designed for this series of the thermodiffusion experiment on board the ISS due to some technical problems of heat transfer in the system.

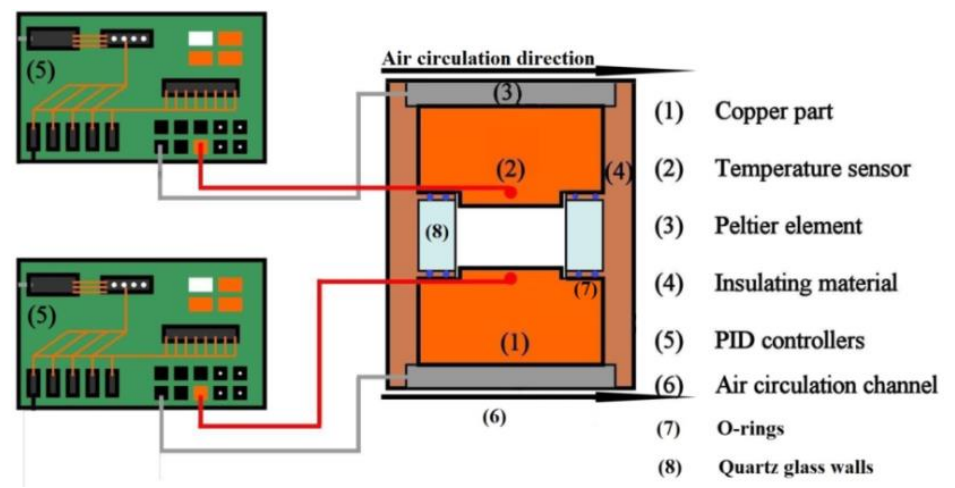

Figure 8-5: Experimental cell and temperature controller sketch[95]

\subsubsection{Experimental procedure for ternary system}

The sample is monitored using a Mach-Zehnder interferometer at two wavelengths. Two laser beams of constant frequency with wavelengths $\lambda=670 \mathrm{~nm}$ and $\lambda=935 \mathrm{~nm}$ are expanded and 
divided into two beams. One beams then travels through the cell holder and the experimental cell while the other bypasses the cell and is used as reference. Figure 8-6a and b show two different paths inside the cell holder. One of the paths follows the beam through the front of the cell (in the direction perpendicular to the thermal gradient) and the other one passes through the void area to be used as the reference beam. The first step to a measurement was thermalization of the sample at the mean temperature to initialize the mixture characteristics by reaching uniform concentration and temperature inside the cavity. The mean temperature that was achieved between the walls of the cell was $25^{\circ} \mathrm{C}$ for half of the DSC experimental runs (which are considered in this study) and $40^{\circ} \mathrm{C}$ for the other half. During the first ninety seconds of this phase, no images were acquired. This step was performed while the experimental run on the previous cell was still ongoing in order to reduce experiment duration.

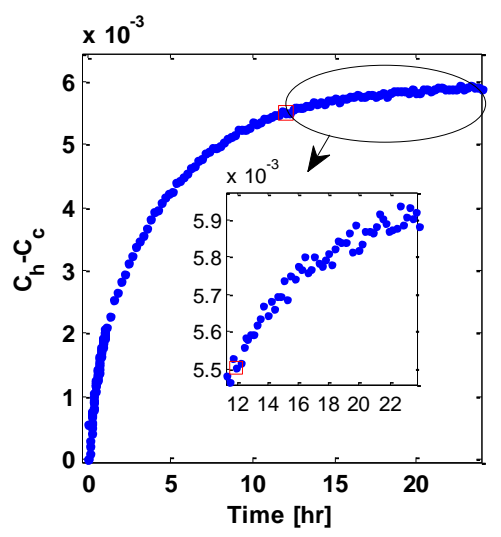

(a) IVIDIL, Ext1-water

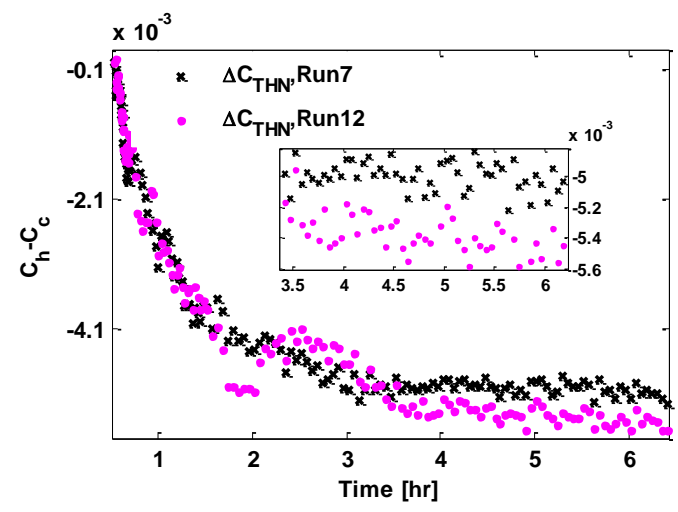

(b) DSC-Run7 and 12-THN

Figure 8-6: Separation versus time during thermodiffusion phase

Then, with temperature gradient buildup, a temperature difference was established across the sample. The process lasted 6 to 8 hours depending on whether the run was normal or extended, respectively. The set point temperature reached the prescribed stability within three minutes (first thermal time) from the imposition of the temperature difference. MZI images and the Peltier elements' temperature corresponding to this phase were acquired. After thermodiffusion was completed, the temperature difference was removed, and the cell was brought back to the mean temperature within three minutes (second thermal time). Then, the data for the active cell were acquired for 4 to 6 hours. After the completion of each run, the system was brought to the initial isothermal and homogeneous concentration condition. If needed, the run could be repeated at a 
different temperature difference. Then, the moveable optics was relocated to another sample and the entire cycle was repeated.

During the thermal time period, the influence of diffusion due to thermal gradients on the refractive index is negligible because the effect of the thermal contrast factor is two orders of magnitude larger than that of the concentration contrast factor $[131,152,153]$. In addition, there is a characteristic diffusion time that is similar to the thermal time as it is also a function of the cell length: $\tau_{C} \propto \frac{L^{2}}{D}$, where $\mathrm{D}$ is the smallest eigenvalue of the diffusion matrix. By definition, at the end of the diffusion time the linear separation of species has been reached. Although 12 hours of thermodiffusion time was not enough for an alcohol mixture of water-isopropanol to reach steady state, the diffusion time in this series of experiments was at most 6 hours, as shown in Figure 8-6. The first part of this figure illustrates the separation of the alcohol mixture that shows noticeable separation and slope of concentration variation at the end of the diffusion time. One can compare the value of the separation after $12 \mathrm{~h}$ and $24 \mathrm{~h}$, which shows noticeable underestimation of the Soret coefficient at $12 \mathrm{~h}$, implying that it had not yet reached steady state. However, the second part demonstrates intensive small fluctuations during the last 2 hours of the separation phase that are caused by optical measurement error sources.

\subsection{Equations Development to Study the Ternary System}

The diffusive mass transport in a ternary mixture in the presence of a temperature gradient is described by three fluxes: one as a result of the temperature difference and two independent mass fluxes. There is no need to define the third mass flux for the last component because the final closing relation describes the third mass flux completely. Given, $\mathrm{i}=1,2$, and 3, the mass fluxes along the direction of the thermal gradient are given by the equations in below:

$$
\begin{aligned}
& \vec{J}_{1}=\rho\left(D_{11} \nabla c_{1}+D_{12} \nabla c_{2}+D_{T 1} c_{1}\left(c_{2}+c_{3}\right) \nabla T\right) \\
& \vec{J}_{2}=\rho\left(D_{21} \nabla c_{1}+D_{22} \nabla c_{2}+D_{T 2} c_{2}\left(c_{3}+c_{1}\right) \nabla T\right) \\
& \sum_{i=1}^{3} \vec{J}_{i}=0
\end{aligned}
$$


where $\rho$ donates the density of the mixture, $c_{i}$ shows the mass fraction of the $i^{\text {th }}$ component, $D_{i i}$ is the pure diffusion coefficients, with $\mathrm{i} \neq \mathrm{j}$ the cross diffusion coefficients in porous media and $D_{T, i}$ represents the thermodiffusion coefficients of the components in the ternary mixture. Mass conservation equations, with previous phenomenological equations as well as the assumption of the convection less regime, allow writing of the following equations that govern the time evolution of the component concentration along the thermal gradient:

$\frac{\partial c_{1}}{\partial t}=\rho\left(D_{11} \nabla^{2} c_{1}+D_{12} \nabla^{2} c_{2}+D_{T 1} c_{1}\left(c_{2}+c_{3}\right) \nabla^{2} T\right)$

$\frac{\partial c_{2}}{\partial t}=\rho\left(D_{21} \nabla^{2} c_{1}+D_{22} \nabla^{2} c_{2}+D_{T 2} c_{2}\left(c_{3}+c_{1}\right) \nabla^{2} T\right)$

$\sum_{i=1}^{3} \frac{\partial c_{i}}{\partial t}=0$

If one neglects the cross-diffusion coefficients in comparison with the pure diffusion coefficients, the concentration difference between the hot and cold sides of each component can be simplified and rewritten as a function of time, as given below:

$$
\begin{aligned}
& \Delta C_{1}(t)=C_{1}(t, L)-C_{1}(t, 0)=S_{T 1} c_{1}\left(c_{2}+c_{3}\right) \Delta T\left[1-\frac{8}{\pi^{2}} \sum_{n, \text { odd }}^{\infty} \frac{1}{n^{2}} \exp \left(-n^{2} \frac{t}{\tau_{D_{11}}}\right)\right] \\
& \Delta C_{2}(t)=C_{2}(t, L)-C_{2}(t, 0)=S_{T 2} c_{2}\left(c_{3}+c_{1}\right) \Delta T\left[1-\frac{8}{\pi^{2}} \sum_{n, o d d}^{\infty} \frac{1}{n^{2}} \exp \left(-n^{2} \frac{t}{\tau_{D_{22}}}\right)\right] \\
& \sum_{i=1}^{3} C_{i}=1
\end{aligned}
$$

where $\mathrm{L}$ is the length of diffusion. $S_{T 1}=\frac{D_{T 1}}{D_{11}}$ and $S_{T 2}=\frac{D_{T 2}}{D_{22}}$ represent Soret coefficients of the ternary mixture. Using only one laser source provides one equation, as below, that has two unknowns during the diffusion time: $\Delta C_{1}$ and $\Delta C_{2}$.

$$
\Delta n_{1}(x, y)=\left(\frac{\partial n_{1}}{\partial T}\right)_{T_{0}, C_{0}, \lambda_{1}} \Delta T(x, y)+\left(\frac{\partial n_{1}}{\partial c_{1}}\right)_{T_{0}, C_{2}, \lambda_{1}} \Delta C_{1}(x, y)+\left(\frac{\partial n_{1}}{\partial c_{2}}\right)_{T_{0}, C_{1}, \lambda_{1}} \Delta C_{2}(x, y)
$$


where, $\Delta T(x, y)$ and $\Delta C_{1}(x, y)$ are the temperature and concentration changes at the point $(\mathrm{x}, \mathrm{y})$, respectively. $(\partial n / \partial T)$, which is generally two orders of magnitude smaller than the concentration contrast factor.

For example, $\left(\partial n_{2} / \partial c_{1}\right)_{p_{0}, T_{0}, C_{3}, x_{2}}$ means the rate of change of the refractive index with a change in the concentration of the first component in the condition of initial pressure, temperature, and constant mass fraction of the third component while the second laser is being used. Consequently, it is almost impossible to solve two unknowns with one equation. Because of this limitation, a second laser source of different wavelength, with specific kinetics for each wavelength, makes it possible to obtain $\Delta C_{1}$ and $\Delta C_{2}$. It is noted that $\Delta C$ in this work represents the maximum separation between hot and cold sides.

$\Delta n_{2}(x, y)=\left(\frac{\partial n_{2}}{\partial T}\right)_{T_{0}, C_{0}, \lambda_{2}} \Delta T(x, y)+\left(\frac{\partial n_{2}}{\partial c_{1}}\right)_{T_{0}, C_{2}, \lambda_{2}} \Delta C_{1}(x, y)+\left(\frac{\partial n_{2}}{\partial c_{2}}\right)_{T_{0}, C_{1}, \lambda_{2}} \Delta C_{2}(x, y)$

By adjusting the amplitudes of the each laser and combining this obtained data with the values of the pure diffusion coefficients, it is be possible to calculate $D_{T 1}$ and $D_{T 2}$. In this case, because the diffusion matrix of the mixture with this specific composition may not be found in literature, these coefficients had to be estimated using the same experiment itself. Either the results of the second phase of the experiment in an isothermal condition or curve fitting using the analytical solutions could be used to calculate the coefficients. Thus, the calculation of the diffusion coefficients deals with at least four to six unknown parameters that need to be estimated using curve-fitting methods.

\subsection{Data Processing Methodology for Ternary System}

The governing principle that is used in this study is a combination of different Fourier Transform techniques. As illustrated in Figure 8-7, the procedure established for image processing is as follows: after downloading the raw data and converting both MR and MN binary files to digital bitmap images (see Figure 8-7a,b), each image is first subjected to resizing, cropping and then applying the phase extraction method. 


\subsubsection{Image Processing Procedure}

As described earlier, there are two lasers with different wavelengths (670 nm and $935 \mathrm{~nm})$ inside the moving bridge. The images that are taken by the laser with $\lambda=670 \mathrm{~nm}$ are called MR and those with $\lambda=935 \mathrm{~nm}$ are called MN. In the case of ternary systems, in each loop of image processing, four images must be considered simultaneously.

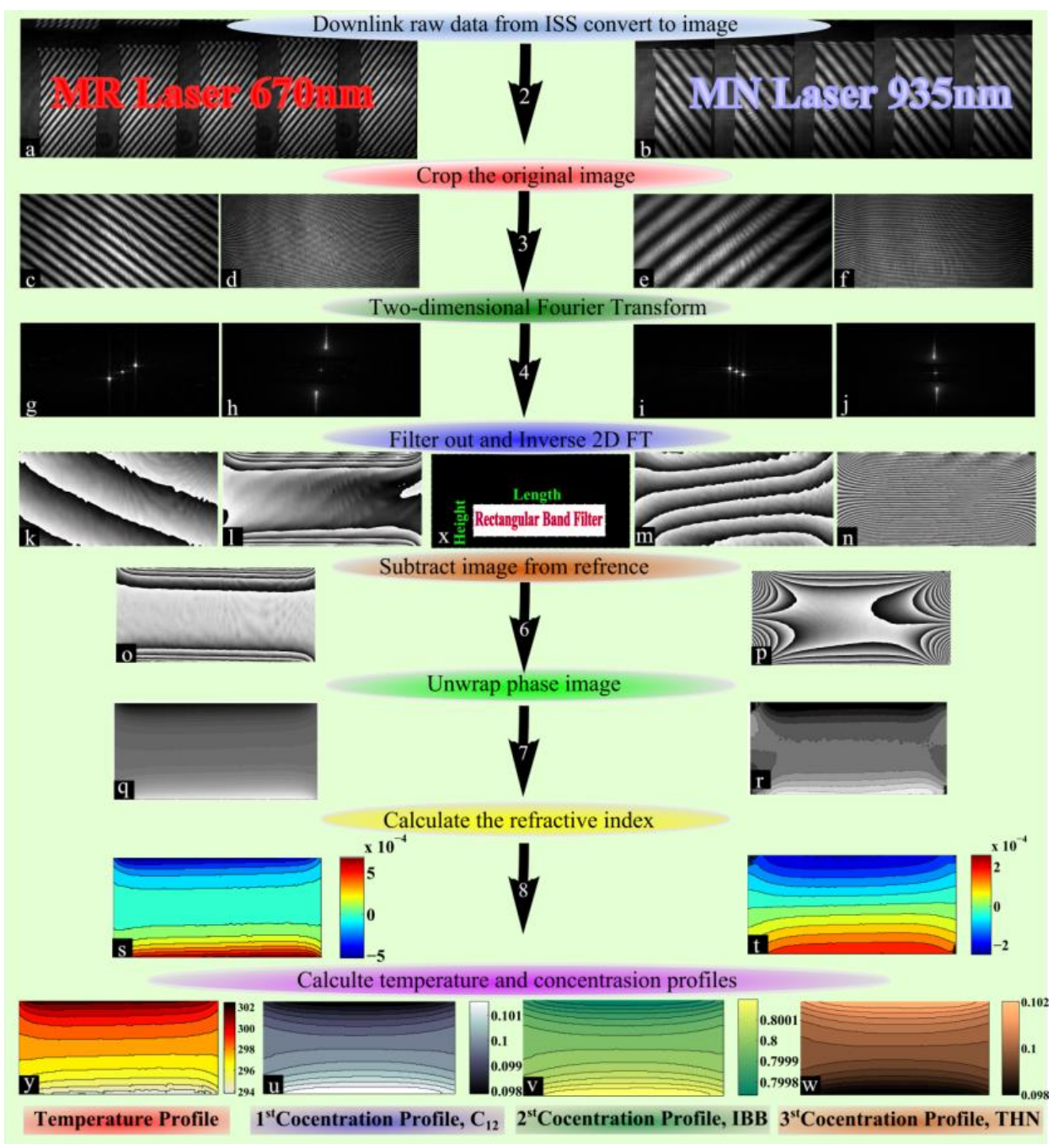

Figure 8-7: Principle scheme of Fourier Transform image processing technique to analyze optical digital interferometry of a ternary system $(\mathrm{a}, \mathrm{b})$ Converted binary MR and MN lasers data, (c,e) Cropped MR and MN reference images, (d,f) Cropped current MR and MN images, (g,h,i,j) Two dimensional FFT magnitude of image in parts $c$, d,e and $\mathrm{f},(\mathrm{k}, 1, \mathrm{~m}, \mathrm{n})$ Inversed 2D FFT magnitude of filtered image in parts g,h,i and j, (o) Subtracted MR and MN images from reference images, $(\mathrm{q}, \mathrm{r})$ Unwrapped phase images, (s,t: Refractive index profiles of MR and MN lasers, $(\mathrm{u}, \mathrm{v}, \mathrm{w})$ Concentration contours of $\mathrm{C}_{12}$, IBB and THN respectively, (x) Filter mask, (y) Temperature contour. 
Two references that remain without any change until the end of the process are employed on normalizing MR and MN images separately. See Figure 8-7c,e which shows representative MR and $\mathrm{MN}$ reference images, respectively. In addition to these two reference images, one MR and one MN image are processed in each loop as shown in Figure 8-7d,f, respectively. Then the twodimensional Fourier transform has been applied to all of the fringe image, as illustrated in Figure $8-7 \mathrm{~g}, \mathrm{~h}, \mathrm{i}, \mathrm{j}$. A basic schematic sketch of the rectangular band filter is demonstrated in Figure 8-7x. A similar procedure is applied to the reference images to evaluate $\phi_{r e f}\left(x, y, t_{\text {ref }}\right)$. Next, reference images are used by subtracting the concurrent MR or MN image from its reference image, which results a wrapped phase difference map. Thus, the phase distribution can expressed as follows by the subtraction of the phase of the $\mathrm{i}^{\text {th }}$ image from the reference image:

$\Delta \phi\left(x, y, t_{i}\right)=\phi\left(x, y, t_{i}\right)-\phi_{\text {ref }}\left(x, y, t_{\text {ref }}\right)$

\subsubsection{Calculation of the Change in Refractive Index}

The refractive index distribution leads to a calculation of temperature and concentration variation in the cell as well as the difference between the hot and cold sides. The relation between phase difference, $\Delta \phi$, and $\Delta \mathrm{n}$ is illustrated below:

$\Delta n(x, y)=n(x, y)-n_{r e f}(x, y)=\frac{\lambda}{2 \pi L} \Delta \phi(x, y)$

When separation in the mixture has reached a stationary point, in order to have an accurate result in this study, the aided Genetic Algorithm (GA) with Fast Robust Discretized Smoothing was implemented to find the curve that best fit that of the experimental separation. Results from GA accurately estimated the Soret and diffusion coefficients simultaneously with a defined objective function. In a steady-state regime, an ideal case where time goes to infinity or reaches the diffusion time, the complex differential transient equations of separation can be simplified and the Soret coefficient can be calculated as follows:

$S_{T i}=\frac{\Delta C_{i, s t}}{C_{i, 0}\left(1-C_{i, 0}\right) \Delta T}$

Both runs lasted $6 \mathrm{~h}$ while possessing a thermal gradient. 


\subsubsection{Experimental accuracy, calculation of temperature and concentration}

\subsubsection{Accuracy of experiment}

During the first phase of the experiment when there is no temperature difference, ideally, no refractive index variation should be observed; however, because of inherent errors in the optical system, some fluctuations with a mean value of zero are recorded. These fluctuations present a possible error in the measurement.

As shown in Figure 8-8, the maximum refractive index variation between the hot and cold walls is less than $2 \times 10^{-5}$ for the MN laser and $1 \times 10^{-5}$ for the MR laser during the isothermal condition. This means that the maximum error of measuring the temperature variation is less than $0.08 \mathrm{~K}$ for the $\mathrm{MN}$ laser, while the MR laser shows a better and more accurate performance with maximum error less than $0.04 \mathrm{~K}$.

\subsubsection{Temperature profile}

During thermal time, that is, less than seven minutes for most hydrocarbon mixtures, it might be an accurate assumption that the change in refractive index represents the temperature change in the domain and therefore the effect of component movement in the mixture can be neglected. Nevertheless, because thermal time is intensively smaller than diffusion time (about six hours in this case), ignoring two or three minutes may not change the final concentration result at the end of diffusion time. Consequently, during thermal time, Equations 8-10 and 8-11 might be rewritten as follows:

$$
\begin{aligned}
& \Delta T(x, y)=\frac{\Delta n_{1}(x, y)}{\left(\frac{\partial n_{1}}{\partial T}\right)_{T_{0}, C_{0}, \lambda_{1}}} \\
& \Delta T(x, y)=\frac{\Delta n_{2}(x, y)}{\left(\frac{\partial n_{2}}{\partial T}\right)_{T_{0}, C_{0}, \lambda_{2}}}
\end{aligned}
$$

Accordingly, the temperature profile in the system can be measured with both lasers separately. These two temperature profiles can be compared to show identical variation of temperature profiles captured by the optical system. 


\subsubsection{Concentration profile}

Initially, the system is in a homogeneous condition and after applying, a temperature gradient the homogeneous condition disturbs. From the images recorded during the first thermal time, the temperature field can be determined, and according to the theory of heat transfer in the liquid system, the temperature profile will not change after thermal time. Consequently, any derivative of temperature is equal to zero and Equations 8-10 and 8-11 can be rewritten as follows:

$$
\begin{aligned}
& \Delta n_{1}(x, y)=\left(\frac{\partial n_{1}}{\partial c_{1}}\right)_{T_{0}, C_{2}, \lambda_{1}} \Delta C_{1}(x, y)+\left(\frac{\partial n_{1}}{\partial c_{2}}\right)_{T_{0}, C_{1}, \lambda_{1}} \Delta C_{2}(x, y) \\
& \Delta n_{2}(x, y)=\left(\frac{\partial n_{2}}{\partial c_{1}}\right)_{T_{0}, C_{2}, \lambda_{2}} \Delta C_{1}(x, y)+\left(\frac{\partial n_{2}}{\partial c_{2}}\right)_{T_{0}, C_{1}, \lambda_{2}} \Delta C_{2}(x, y)
\end{aligned}
$$

So, the mass fraction of the first two components can be calculated as follows:

$$
\left(\begin{array}{l}
\Delta C_{1}(x, y) \\
\Delta C_{2}(x, y)
\end{array}\right)=\left(\begin{array}{ll}
\left(\frac{\partial n_{1}}{\partial c_{1}}\right)_{T_{0}, C_{2}, \lambda_{1}} & \left(\frac{\partial n_{1}}{\partial c_{2}}\right)_{T_{0}, C_{1}, \lambda_{1}} \\
\left(\frac{\partial n_{2}}{\partial c_{1}}\right)_{T_{0}, C_{2}, \lambda_{2}} & \left(\frac{\partial n_{2}}{\partial c_{2}}\right)_{T_{0}, C 1, \lambda_{2}}
\end{array}\right)^{-1}\left(\begin{array}{l}
\Delta n_{1}(x, y) \\
\Delta n_{2}(x, y)
\end{array}\right)
$$

\subsection{Results and Discussion}

The main objective in this chapter is not only to investigate the separation of the components toward different walls and measure the Soret coefficients, but also to study the quality of the SODI apparatus during the thermal and diffusion time to determine the accuracy of the measurement of the thermodiffusion phenomenon using the SODI facilities. For this purpose, the behavior of the mixture is studied inside the cavity domain at different locations and as a function of time close to both the hot and cold walls.

\subsubsection{Refractive index variation}

Figure 8-8 shows the maximum refractive index variation between the hot and cold walls. The most important fact that can be deduced from this figure is the similar smooth behavior of the $\mathrm{MN}$ and MR lasers. In addition to this similarity, if one considers the slight differences in the contrast factors between these two lasers, differences found in the analysis of the data provide a 
sensitive measurement of thermodiffusion separation in the ternary system. In other words, using two lasers must result in two independent equations, with both equations requiring an accurate measurement to distinguish the slight difference between MR and MN beams precisely.

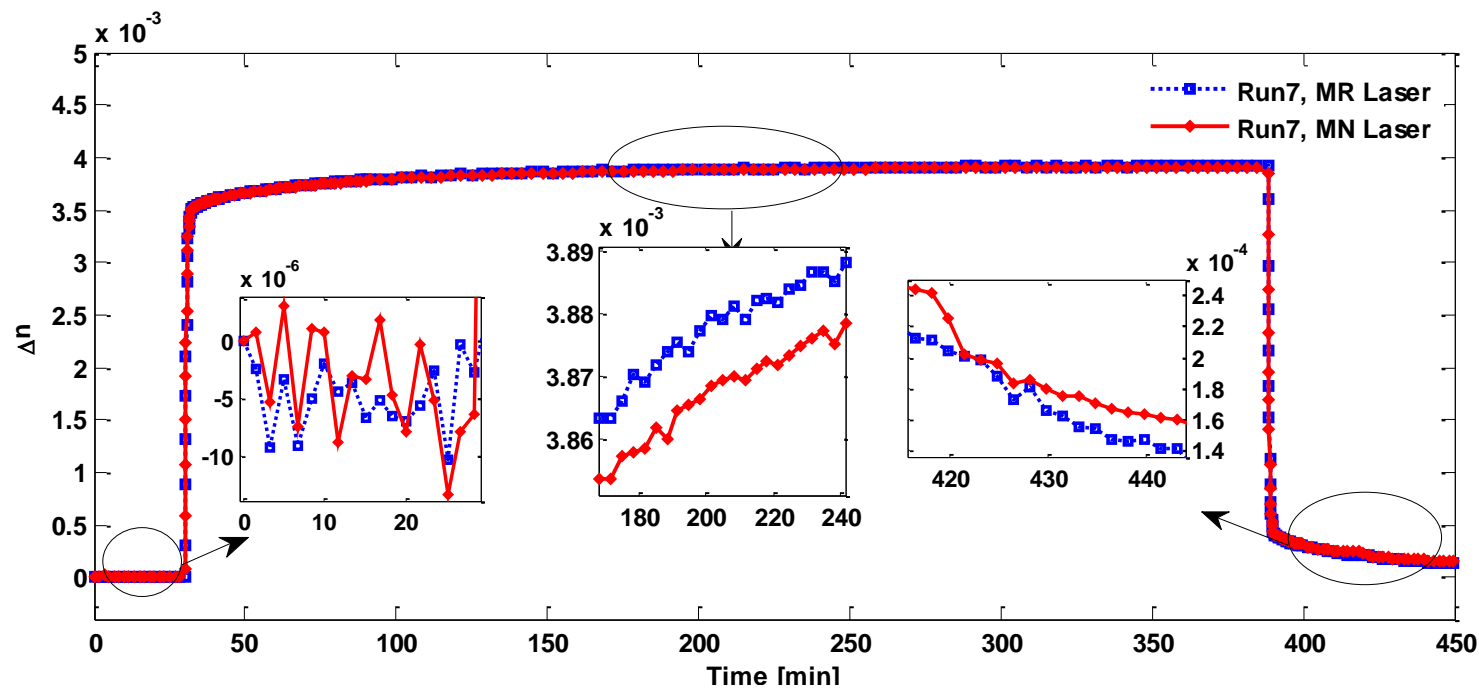

Figure 8-8: Refractive index difference between hot and cold walls versus time during isothermal, thermal and diffusion conditions

Nevertheless, because of using the precision application, which has been developed to analyze the thermodiffusion experiment by means of MZI images and a combination of different Fourier methods, the difference between refractive indices is detected (see magnified plots at the center of Figure 8-8). It must be noted that although this difference is well identified during the thermodiffusion phase, after removing the temperature gradient there are more fluctuations that prevent distinction between the two curves of refractive index variations from each other, especially at several special times such as the period between $420 \mathrm{~min}$ and $430 \mathrm{~min}$. This conflict results in large variations in the concentration calculation in the domain

\subsubsection{Temperature variation}

A linear variation in the thermal gradient is reached in the cavity at the end of thermal time. Liquid properties and the contrast factor at the mean temperature of $298 \mathrm{~K}$ are used to measure the temperature field in the cavity at each pixel of the image. The image yields more than one million data points across the cavity, which has a side area of $0.5 \mathrm{~cm}^{2}$. It is vital to consider the separation as soon as a linear temperature gradient is achieved between the two walls. Figure 8-9 shows the temperature near the top and bottom walls at $120 \mathrm{~s}$, before applying the temperature 
gradient, until the end of thermal time at $200 \mathrm{~s}$. When calculating the temperature field in the cavity experimentally, there were a number of notable points. First, the maximum temperature variation between the hot and cold walls was $8.3 \mathrm{~K}$ in both Run7 and Run12, but Peltier elements inside the copper plates of the cell array recorded a $10 \mathrm{~K}$ difference using the thermal controller system of the apparatus.

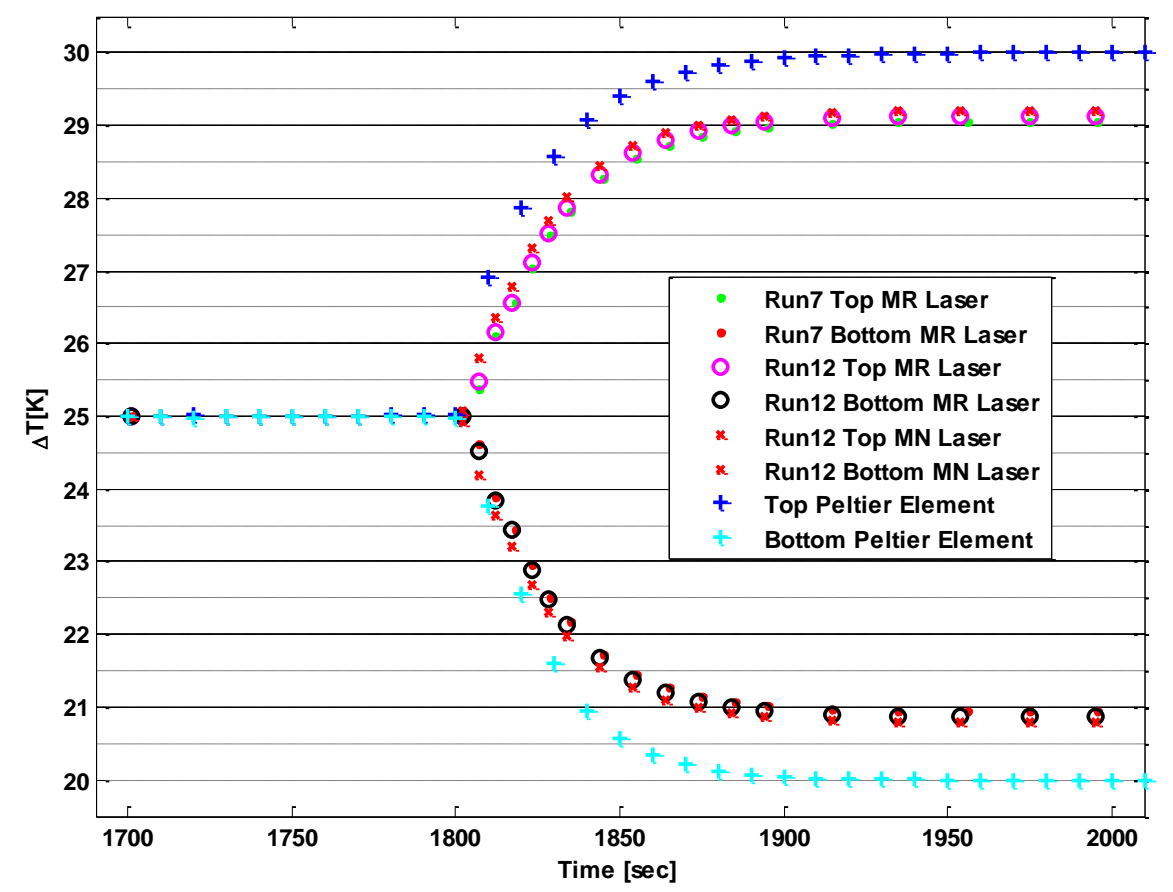

Figure 8-9:Temperature difference between hot and cold sides during thermal time

This difference results from cropping of the images. Cropping the initial images removes the regions where the liquid is in contact with the copper blocks. Inside these areas, optical signals are damaged by a defective alignment of the experimental cell with the optical axis of the interferometer, yielding the reflection of the laser beam from the metal surface [95]. In this study, five percent of pixels that were close to the top and bottom sides of the cavity were removed. However, 90 percent of the height of the cavity with a linear variation of temperature at the end of thermal time should achieve a temperature difference close to $9 \mathrm{~K}$. But, measuring the temperature difference experimentally shows that the maximum difference between the hot and cold walls is less than $8.5 \mathrm{~K}$. Thus, using the Peltier elements to measure the Soret effect might provide underestimated Soret coefficients, because maximum separation is a strong function of the temperature gradient. Moreover, it can be observed that both MR and MN lasers 
in both cases provide their temperature results versus time with less than a $0.07 \mathrm{~K}$ difference. This shows a high accuracy and repeatability of the thermal part of the DSC experiments if all properties of the mixture are measured optically. The maximum error in measurement of the temperature variation is less than $0.1 \mathrm{~K}$ for both wavelengths. This shows the accuracy of this experiment for in both the stability of the temperature variation and its measurement.

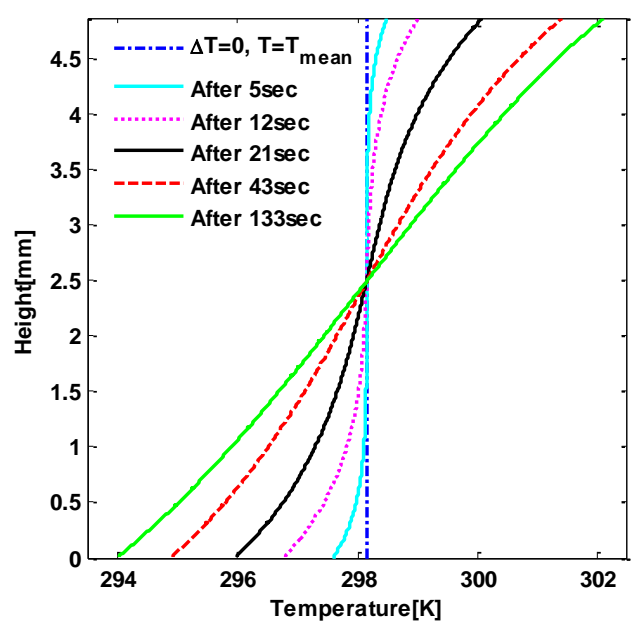

(a) MR laser

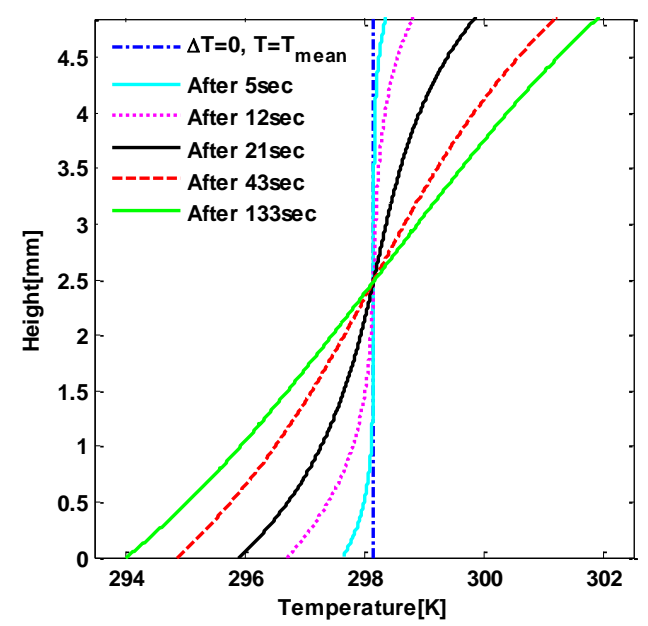

(b) MN laser

Figure 8-10: Temperature variation between hot and cold walls of Run7

Figure 8-10 shows that temperature variations along the direction of the thermal gradient and at the middle of the cavity occur at different times during the thermal-time period. After the thermal time, the curvature of temperature variation along the temperature gradient direction reaches a linear distribution. The curves show identical behaviors of the temperature variation occur at different times for both runs. Figure 8-11 illustrates the temperature field inside the cell at different times.

The approximate linear variation of the temperature in the cell between the cold and hot sides can also be perceived. When the temperature difference is applied to the cell, important deformations of the interference pattern are observed, as shown in Figure 8-11. The temperature field in the liquid measured optically, shows that the thermal controller of the setup induces the development of convection in the liquid as long as a temperature gradient exits across the cell. This convection occurs mainly because the heat fluxes close to the glass walls of the cell where the heat transfer occurs is supposed to insulate the wall. This phenomenon can be recorded by 
observing the phase image after thermal time in general or by subtracting two continuous phase images immediately after thermal time (three minutes after applying temperature) Figure 8-12.

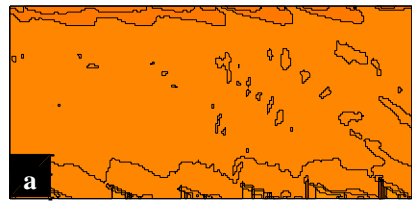

(a) after $1 \mathrm{sec}$

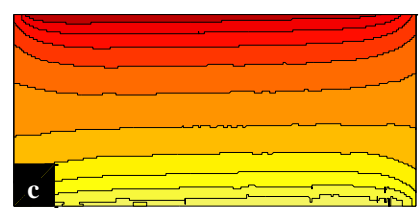

(c) after $41 \mathrm{sec}$
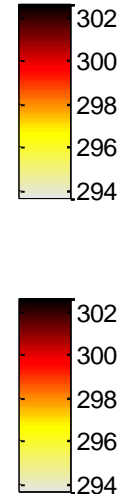

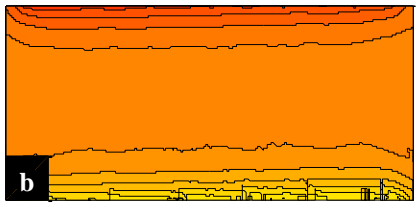

(b) after $5 \mathrm{sec}$

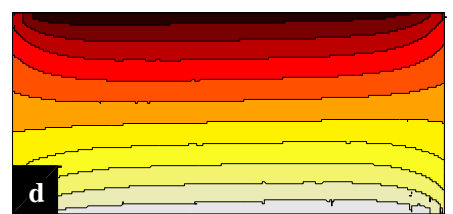

(d) after $133 \mathrm{sec}$

Figure 8-11: Temperature profiles at different times during thermal time, Run12

This interesting observation is clearly shown in Figure 8-12a,b, where numerous bent $2 \pi$ bands are visible close to the lateral walls. These bands should be linear and parallel to the horizontal walls; however, as a result of heat fluxes close to lateral walls the parallel phase map is diminished. These curved bands can change the direction of the heat flux and the point along the lateral walls where the zero heat flux exits. Along the top half of the bands that are close to both lateral walls, it is apparent that each band near the wall has a lower amount of heat transfer (darker shade in Figure 8-12) and toward the inside of the cell there is a higher amount of heat transfer (lighter shade in Figure 8-12).

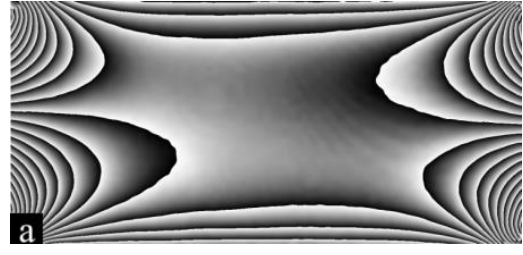

(a) Phase after $1 \mathrm{sec}$

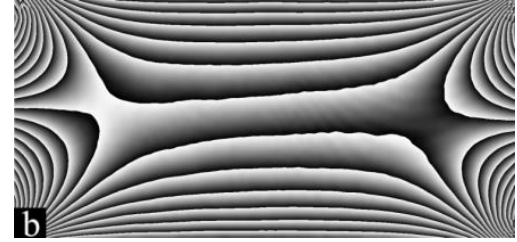

(b) Phase after $6 \mathrm{hr}$

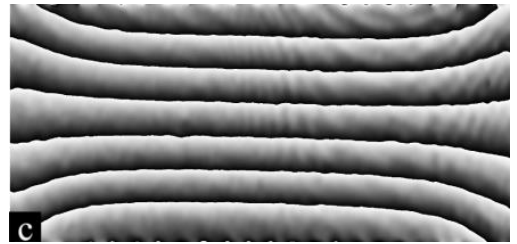

(c) Phase difference of first and last phase

Figure 8-12: Phase and phase difference distributions in the cavity at different times during thermal time, Run12

In other words, there is a gradient in the amount of heat transfer from lower to higher close to the lateral wall in the upper half of the cell. Moreover, a completely different pattern of heat transfer can be observed at the bottom half of the cavity close to the lateral wall, where the heat transfer 
gradient moves from high to low from the glass walls toward the middle of the cell. This means heat transfers from outside the cell near the lateral boundaries, toward the middle of the cavity. Moreover, the point along the vertical walls where the gradient behavior from low to high changes to high to low is the only point with zero heat flux on the lateral walls. While these facts do not change the thermal performance of the apparatus greatly, it is important not to consider a completely insulated condition close to the lateral walls for further experimental or numerical analysis. In order to measure the separation or concentration contour, it is required to crop and consider the region far from the areas where heat fluxes induce small convection cells near the lateral boundaries.

Thermal conductivity of the O-rings that are shown in Figure 8-5 causes the slight lateral deformation of the temperature field visible in Figure 8-12. This effect had been observed in previous studies of the SODI facilities [114,120,152]. From the internal side of the O-rings, the small gap between the copper and quartz was filled by the working liquid. On the external side, the gap was filled by surrounding gas that acts as a poor heat conductor $[114,120,152]$. Consequently, the largest thermal gradient arises at the corners, due to the relatively high heat conductivity of the rubber sealing and surrounding regions. This is shown in detail in Figure 8-11d.

\subsubsection{Diffusion Time and Concentration Profile}

Despite the nonlinear temperature variation at the corners, taking the reference image at the end of thermal time (Figure 8-12a) and subtracting the image at the end of the thermodiffusion phase (Figure 8-12b), the influence of heat fluxes could be removed from the domain (see Figure 8-12c). On the other hand, because of the nonlinear temperature distribution at the corners, a nonlinear phase map resulted, as shown in Figure 8-12c.

This map illustrates some deviance from pure separation close to the lateral walls. In order to ignore this part of the cell in the Soret coefficient measurements, all images were re-cropped and processed such that only the middle part of the cell is considered for the estimation. The maximum concentration difference corresponds to both cases can be extracted from the chart shown in Figure 8-13. The smooth pattern of separation of THN toward the cold side of the cell versus time is characteristic of a positive Soret effect and is consistent in both Run7 and Run12. 
There was almost identical separation observed for IBB, although it separates toward the hot wall and shows a negative Soret effect. While there are some fluctuations in the separation of IBB in Run7 after two hours of the thermodiffusion phase, the separation of $\mathrm{C}_{12}$ behaves identically for both runs.

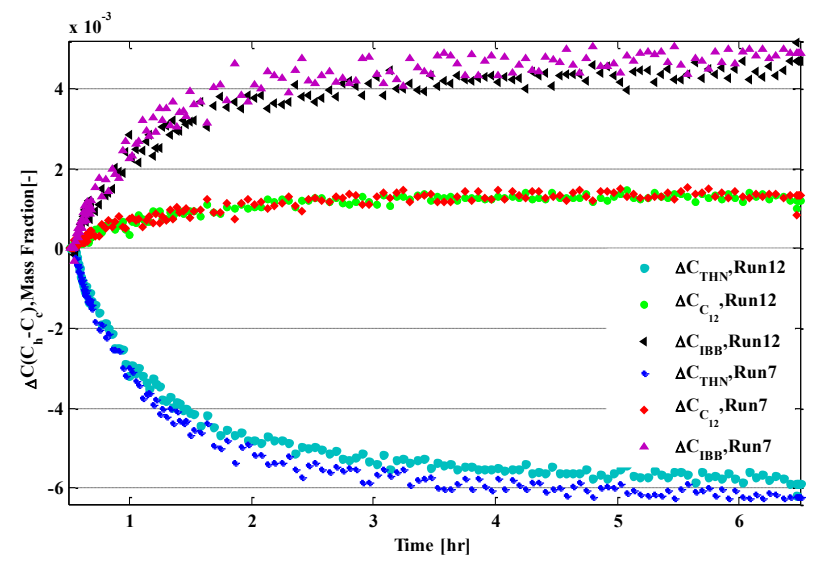

Figure 8-13: Separation between hot and cold sides during thermo-diffusion time

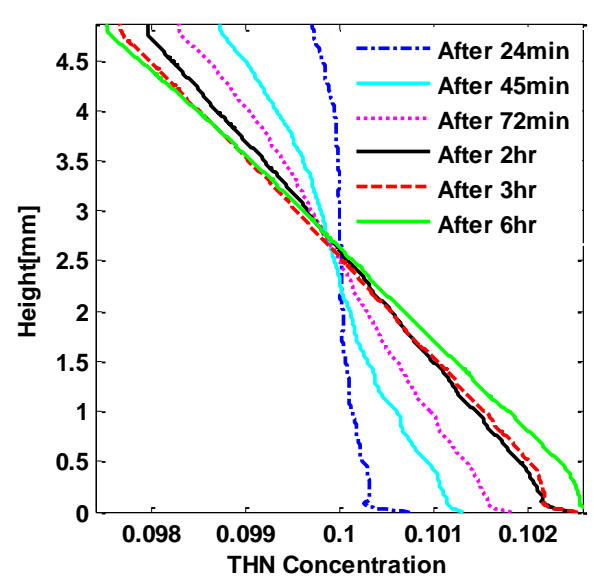

(a) Run7

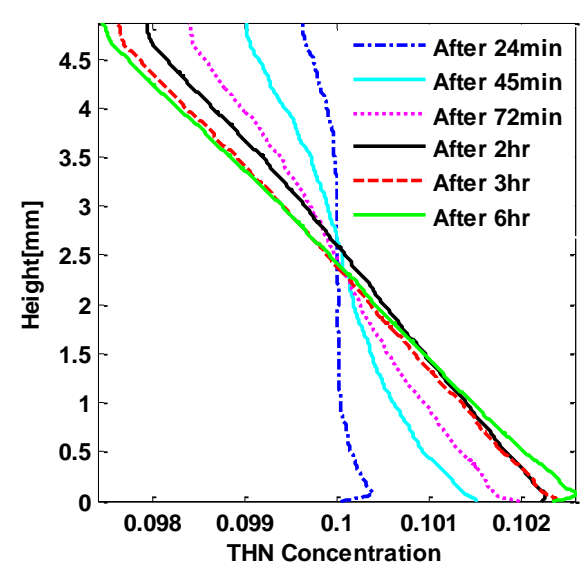

(b) Run12

Figure 8-14: THN concentration variation between hot and cold walls at various times

The separation magnitude of both THN and IBB are similar, while a closer examination of the results in Table 16 reveals that the separation of THN is stronger. This means that the third component moves toward the hot side weakly. Despite this weaker separation of $\mathrm{C}_{12}$, which can be barely detected by MZI, $\mathrm{C}_{12}$ shows a negative thermodiffusion coefficient in this mixture at a 
mean temperature of $298 \mathrm{~K}$. The similar the separation of the components for both runs demonstrates the repeatability and accuracy of digital optical interferometry not only to calculate Soret coefficients for binary systems, but also to measure them in ternary systems.Figure 8-14 and Figure 8-15 show the concentration variations of THN and IBB, respectively, along the direction of the thermal gradient at the middle of the cavity at several times during the diffusion period corresponding to four runs.

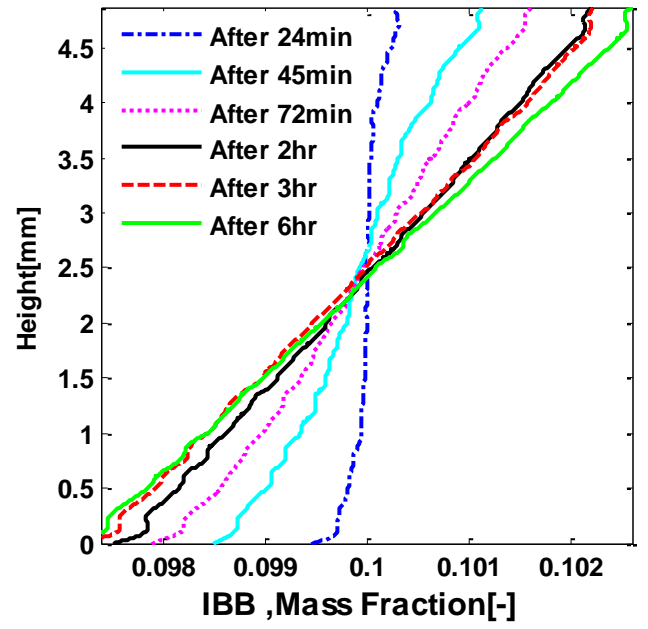

(a) Run7

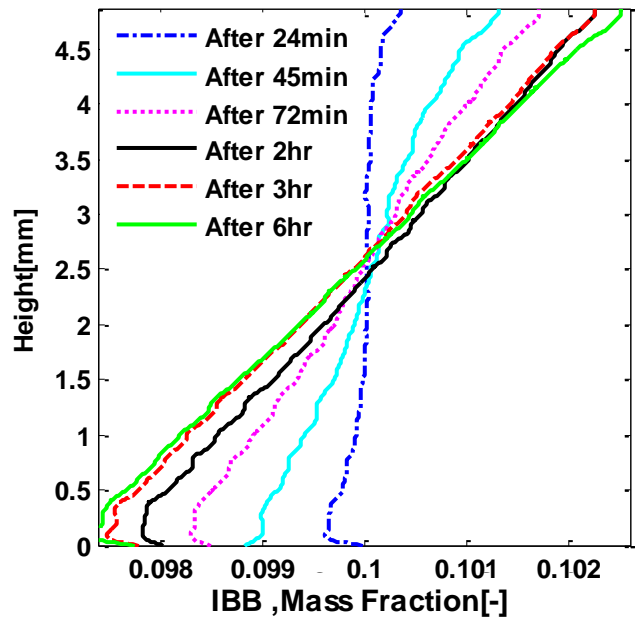

(b) Run12

Figure 8-15: IBB concentration variation between hot and cold walls at various times

This figure compares the quality of separation inside the cell far from the boundaries at various times. Despite the small amount of separation during the first hour of the thermodiffusion phase, the separation starts close to the hot and cold walls with a linear separation variation from the top to the bottom of the cavity. This is observed in both runs. It can be concluded that the ISS provides a suitable environment for this experiment.

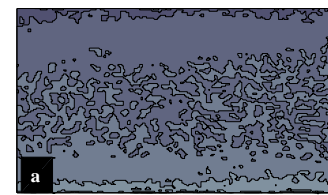

(a) after 30min

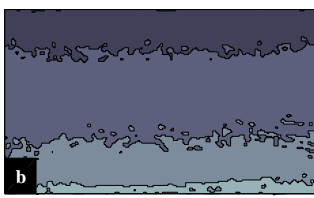

(b)after $75 \mathrm{~min}$

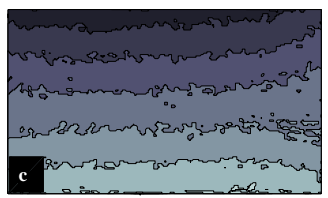

(c) after $3.5 \mathrm{~h}$

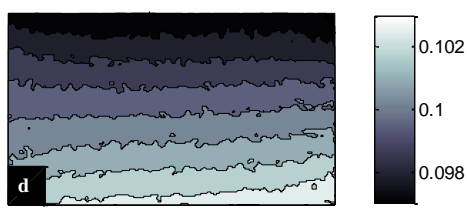

(d) after $6 \mathrm{~h}$

Figure 8-16: THN concentration at different times, Run12 
According to Figure 8-14 and Figure 8-15, after four to five hours at steady state, a stable separation is achieved. Thus, as shown in Figure 8-6, in contrast with the IVIDIL experiments, which needed more than $22 \mathrm{~h}$ to attain a linear variation of separation at a steady-state condition and $12 \mathrm{~h}$ devoted for thermodiffusion, enough time was provide for the thermodiffusion phase in the present study. The measurement of ternary mixture via MZI is highly sensitive to errors for a few reasons. First is the weak separation of one of the components. As a result of this small separation, a small processing error always arises. The second reason is the close performance and contrast factors of the MN and MR laser for this test mixture, which required the use of a sensitive image processing procedure.

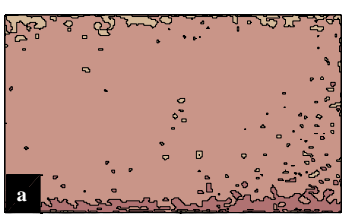

(a) after 30min

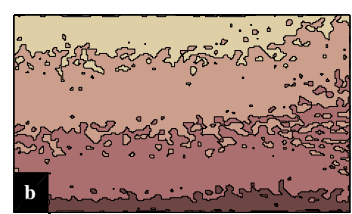

(b)after $75 \mathrm{~min}$

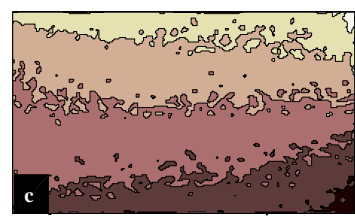

(c) after $3.5 \mathrm{~h}$

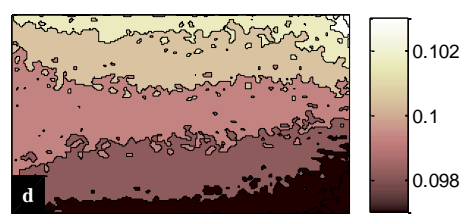

(d) after $6 \mathrm{~h}$

Figure 8-17: IBB concentration profiles in the cavity at different times during diffusion time, Run12

The measurement of ternary mixture via MZI is highly sensitive to errors for a few reasons. First is the weak separation of one of the components. As a result of this small separation, a small processing error always arises. The second reason is the close performance and contrast factors of the MN and MR laser for this test mixture, which required the use of a sensitive image processing procedure.

The third is the image processing calculation error. In this study, one the most accurate processing methods was employed to analyze the experiment, but it is impossible to remove the calculation error for these runs when the separation is close to zero. The last reason is the quality of the images, which are not as good as those from the IVIDIL experiment, as a result of having dust on the front glass that disturbs a clear view of the cell. Figure 8-16 and Figure 8-17 show concentration contours of THN and IBB, respectively, during thermodiffusion time. Visible separation of THN is evident after $30 \mathrm{~min}$; however, as Figure 8-16a shows, there is a mostly uniform concentration of IBB in the domain. Thus, a noticeable IBB separation starts with a delay in comparison with THN. As discussed earlier, the separation starts near the cold and hot 
walls, while after some time separation occurs far from the boundaries and dominates the separation close to the walls.

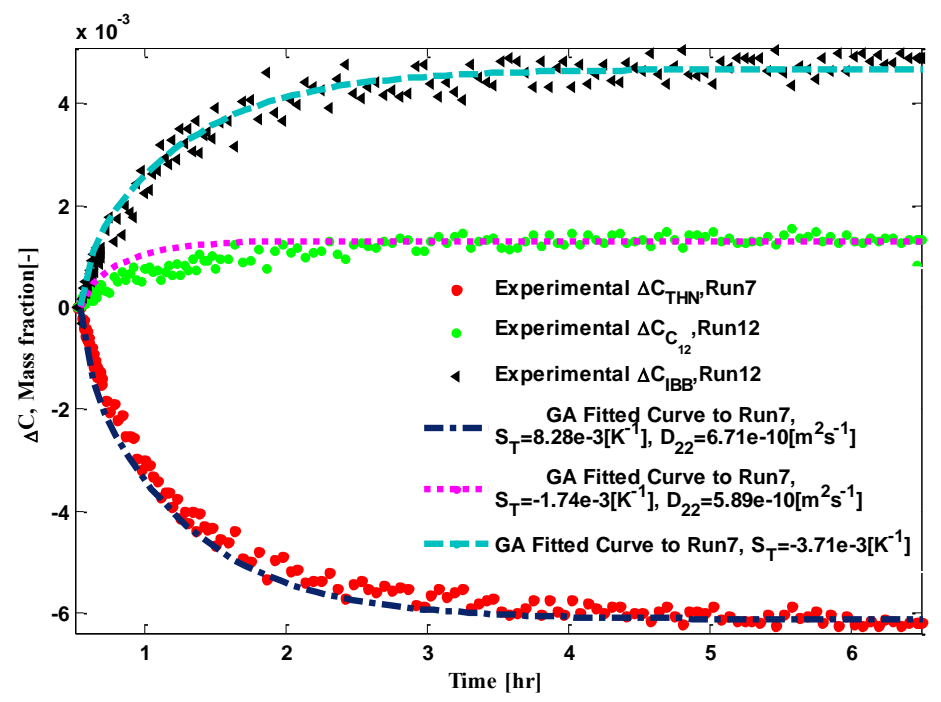

Figure 8-18: Transient experimental separations of Run12, associated with best fitted curve Regardless of the weak amount of separation of THN and IBB during the first two hours of the experiments, after this time there is noticeable separation of these two components, as shown in Figure 8-16d and Figure 8-17b. As shown in Table 15, the lighter component moves toward the cold side. While the molecular mass of THN and IBB are close, the heavier one, IBB, experienced a separation toward the hot side. Finally, THN, which has an intermediate molecular mass, strongly separated toward the cold wall.

\subsubsection{Soret Coefficient, $S_{T}$}

In contrast with binary mixtures, there are limited analyses done in cases in which multicomponent mixtures are considered. According to the literature, this is the first time that Soret coefficients of this mixture with this specific composition have been reported. Thus the Soret coefficients that were estimated with the GA algorithm for both runs are compared to the experimental.

The values of the Soret coefficients, maximum separations, and other information on Run7 and Run12 for this test mixture can be found in Table 16. The values in this table show that both runs have similar results with only some small differences. 
Table 16: DSC Runs7 and 12 experimental results with 10K temperature gradient onboard ISS

\begin{tabular}{|c|c|c|c|c|c|c|c|c|c|c|c|}
\hline & \multicolumn{3}{|c|}{$\begin{array}{c}\Delta \mathrm{C}_{\text {max }} \times 10^{3} \\
\left(c_{h}-c_{c}\right)\end{array}$} & \multicolumn{2}{c|}{$\frac{\Delta \mathrm{c} \times 10^{3}}{\mathrm{c}_{0}\left(1-\mathrm{c}_{0}\right) \Delta \mathrm{T}} \mid\left[\mathrm{K}_{\mathrm{t}=6 \mathrm{hr}}^{-1}\right]$} & \multicolumn{2}{c|}{ Fitted $S_{T} \times 10^{3}\left[K^{-1}\right]$ and } \\
$D \times 10^{10}\left[\mathrm{~m}^{2} \mathrm{~s}^{-1}\right]$ via GA & \multicolumn{2}{|c|}{$\Delta \mathrm{T}_{\max }[\mathrm{K}]$} \\
\hline RUN\# & THN & IBB & $\mathrm{C}_{12}$ & $\mathrm{THN}$ & $\mathrm{IBB}$ & $\mathrm{C}_{12}$ & $\mathrm{THN}$ & $\mathrm{IBB}$ & $\mathrm{C}_{12}$ & $\mathrm{MR}$ & $\mathrm{MN}$ \\
\hline Run 7 & -6.20 & 4.89 & 1.18 & 8.26 & -3.65 & -1.75 & 8.28 & -3.71 & -1.74 & 8.29 & 8.34 \\
\hline Run12 & -5.89 & 4.70 & 1.32 & 7.86 & -3.52 & -1.79 & 8.19 & -3.68 & -1.82 & 8.33 & 8.37 \\
\hline
\end{tabular}

According to the estimated Soret coefficients in both cases, which are presented in Table 16, similar values for $\mathrm{S}_{\mathrm{T}}$ were found for all components at both six hours into steady state and using the curve-fitting method. However, the values of GA show a slightly greater value of $S_{T}$, which means that although the steady-state condition is reached; there is still a small ratio of separation that separates the components in the system. The average values of Soret coefficients of THN, $\mathrm{C}_{12}$, and IBB are reported as $-8.24 \times 10^{-3},-1.88 \times 10^{-3}$ and $-3.69 \times 10^{-4}$, respectively.

Comparing the GA results with experimental data was performed and the results are shown in Figure 8-18. Moreover, the best GA solutions corresponding to these two experimental runs are shown plotted versus time in Figure 8-18. It can be seen that these curves coincide well, especially for THN and $\mathrm{C}_{12}$, which have a noticeable separation trend. These matched curves show the good performance of this method to estimate diffusion coefficients.

\subsection{Conclusions}

The main goal of this chapter, which was to determine the Soret coefficients of THN-IBB-C 12 mixture with initial mass fractions of $0.10,0.80$, and 0.10 , respectively, was achieved. The accuracy and problems with the quality of the SODI apparatus on board the ISS have been discussed. For this purpose, Run7 and Run12, according to the DSC timetable, with a predicted $10 \mathrm{~K}$ temperature difference were analyzed in detail. Mathematical analysis by means of Fourier transform image processing and a curve-fitting method using GA are employed to obtain results that show an accurate fitting result.

The collected experimental results of DSC showed a linear temperature field inside the cell, particularly in the central section, while slight deformations of the temperature field at the walls of the cell were observed. These distortions are caused by several convective cells being 
generated. These convection cells exist because of heat fluxes close to the lateral walls, which should have been insulated in order to prevent the formation of convection cells. It was found that measuring the temperature difference experimentally and with MZI has a direct effect on the calculated Soret coefficients. Although there are some nonlinear behaviors in the temperature gradient at the corners of the domain, they do not affect the linear temperature and separation variation at the center of the cell and along the thermal gradient.

It is shown that despite the IVIDIL experiment, six hours of thermodiffusion time provides enough time to reach steady state in the separation of this particular mixture, including a barely noticeable amount of separation during the last three hours of the experiment. The correspondence between the experimental data and the fitted curves from the genetic algorithm that estimated four unknowns simultaneously is observed. Finally, THN with an intermediate molecular mass strongly separated toward the cold wall. This proves that the MZI not only allows measuring the diffusion coefficients in binary systems with high precision, but it also can be used to analyze multicomponent systems. Reaching this goal requires employing different lasers not only with different wavelengths but also with different contrast factors on the test mixture. Using two lasers with mostly similar properties corresponding to the test mixture was the only point that provided difficulties in processing the results. Moreover, the entire calculation is a function of the accurate measurement of the contrast factors. In short, the ISS provides a suitable environment for this experiment and results of the DSC cell number two were found to be clear and reliable. 


\section{Chapter 9: Soret and Diffusion Coefficients Measurement of Ternary Mixtures of Dodecane, Isobutylbenzene and 1,2,3,4-Tetrahydronaphthalene}

\subsection{Introduction}

Thermodiffusion or Soret effect is a heat and mass transfer phenomenon in a non-isothermal liquid, gas or even solid mixture due to a local temperature gradient $[2,63,64]$. This phenomenon is more pronounced in oil fields, usually due to the porous environment $[4,5,41,156]$. Oil exploration models and simulations, work on million-years scale while improved oil recovery models work on decades scale[9]. According to this fact, the impacts of a weak or slow mass transfer phenomenon such as thermal diffusion or mass diffusion must be considered in the case of thermal gradient existence along an oil reservoir. Thus an accurate and advanced modeling of mass diffusion is vital in oil exploration and optimal oil recovery.

A precise and better understanding of the thermodiffusion phenomena in multi-component mixtures results in a more accurate modeling of oil reservoirs and it may reduce the number of required wells, which approximately costs about $\$ 20$ million/well. Before this observation, Soret coefficient values were usually ignored in oil recovery investigation [27]. Thermodiffusion also has critical applications in environment discovery [11], microfluidic applications [12,13], particle motion in microfluid devices[14] and life science sector in the field of DNA studies[1517].

\subsection{Chapter Aims:}

In current chapter, we reported the results of thermodiffusion experiment for a ternary mixture of THN-IBB-C12 for five different compositions. The results of this experiment can be used as references for the ground base experiments since the level of gravity and induced convection inside the cell were minimized and pure thermodiffusion level has been achieved. Twenty cases taking more than $200 \mathrm{hr}$ of space experiment are considered here for five different compositions. 
While the assigned temperature gradient for all cases was $10 \mathrm{~K}$ the mean temperature for half of the cases was $25 \mathrm{C}$ and for the rest it was $40 \mathrm{C}$.

\subsection{Mass Fluxes in Ternary Mixture in the Presence of $\Delta \mathbf{T}$}

The diffusive mass transport in a ternary mixture along with the application of temperature gradient and at constant distribution can be explained by three fluxes: two independent mass fluxes (there exits the third component of mass flux but it depends on the first two fluxes) and one owing to the temperature difference. The mass flux of any component can be represented using three sources of mass flux as follow:

$\vec{J}_{1}=-\rho\left(D_{11} \nabla c_{1}+D_{12} \nabla c_{2}+D_{T 1} c_{1}\left(c_{2}+c_{3}\right) \nabla T\right)$

$\vec{J}_{2}=-\rho\left(D_{21} \nabla c_{1}+D_{22} \nabla c_{2}+D_{T 2} c_{2}\left(c_{3}+c_{1}\right) \nabla T\right)$

$\sum_{i=1}^{3} \vec{J}_{i}=0$

Accordingly, the concentration difference between the hot and cold sides for each component can be simplified functions of time, diffusion and Soret coefficient, as follows[27]:

$$
\begin{aligned}
& \Delta C_{1}(t)=C_{1}(t, L)-C_{1}(t, 0)=S_{T 1} c_{1}\left(c_{2}+c_{3}\right) \Delta T\left[1-\frac{8}{\pi^{2}} \sum_{n, \text { odd }}^{\infty} \frac{1}{n^{2}} \exp \left(-n^{2} \frac{t}{\tau_{D_{11}}}\right)\right] \\
& \Delta C_{2}(t)=C_{2}(t, L)-C_{2}(t, 0)=S_{T 2} c_{2}\left(c_{3}+c_{1}\right) \Delta T\left[1-\frac{8}{\pi^{2}} \sum_{n, \text { odd }}^{\infty} \frac{1}{n^{2}} \exp \left(-n^{2} \frac{t}{\tau_{D_{22}}}\right)\right] \\
& \sum_{i=1}^{3} C_{i}=1
\end{aligned}
$$

where $\tau_{\mathrm{Dii}}$ is diffusion time. $\mathrm{S}_{\mathrm{T}, \mathrm{i}}$ represents the Soret coefficient of the $\mathrm{i}^{\text {th }}$ component in the ternary mixture.

\subsection{Results and Discussion}

As shown in Figure 9-1, the cell array on-board ISS has six cells. Five of these cells contained ternary mixtures at various compositions that are considered and analyzed here and the last cell 
was assigned for a binary mixture. The percentage of compositions in each cell can be seen in Table 17.

The refractive index, temperature, and concentration variations have been measured inside the cells at various locations using Mach-Zehnder Interferometry that provides a large view over the whole cell. The goal of this chapter is only to measure the Soret and pure diffusion coefficients of these ternary mixtures at various mean temperatures. It is worth noting that comprehensive studies on the quality of the SODI apparatus during the thermal and mass diffusion times to determine the accuracy of their measurement of thermodiffusion have been done in previous chapter. Figure 9-1 illustrates the schedule of the assembled apparatus, which consists of three parts.

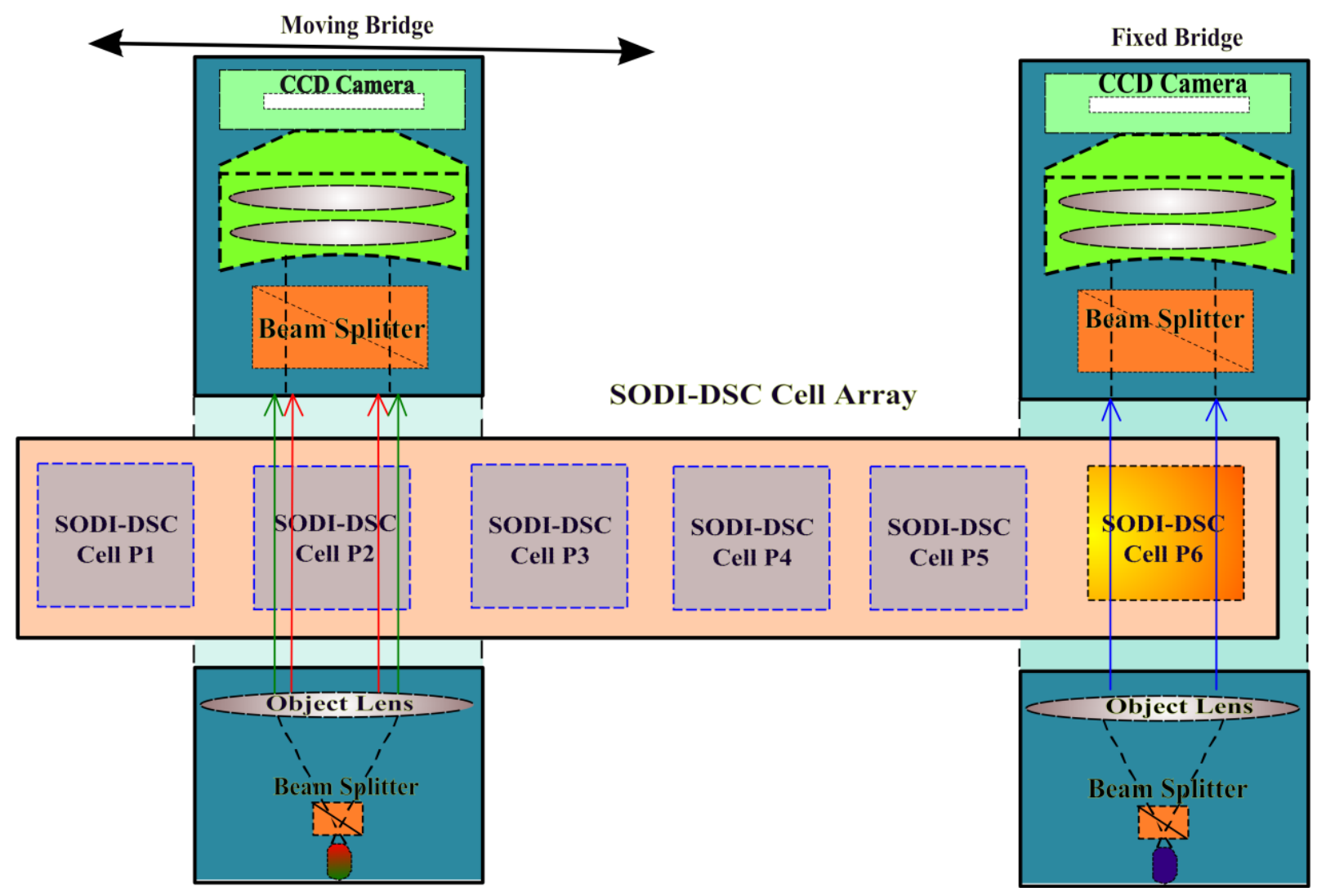

Figure 9-1: Scheme of the assembled equipment along with moving and fix bridge and the DSC cell array

The cell array is designed to minimize the heat transfer from lateral sides of the cells; however, detailed experimental analysis showed that there exists a strong heat transfer from each lateral 
side of the cells[27,144]. Thus, it is concluded that in order to obtain an accurate measurement of Soret effect the region close to lateral walls must be removed from the post processing of the result.

Table 17: Compositions of the ternary mixture in different cells inside the cell array

\begin{tabular}{|c|c|c|c|}
\hline \multirow{2}{*}{ Cell Number } & \multicolumn{3}{|c|}{ Initial mass fraction [wt] } \\
\cline { 2 - 4 } & THN & IBB & $\mathrm{C}_{12}$ \\
\hline 1 & $10 \%$ & $10 \%$ & $80 \%$ \\
\hline 2 & $10 \%$ & $80 \%$ & $10 \%$ \\
\hline 3 & $80 \%$ & $10 \%$ & $10 \%$ \\
\hline 4 & $45 \%$ & $10 \%$ & $45 \%$ \\
\hline 5 & $40 \%$ & $20 \%$ & $40 \%$ \\
\hline
\end{tabular}

Consequently, only 50 percent of the length of the cell $(\sim 5 \mathrm{~mm})$ and at the middle region of the cell is considered in this study; see Figure 9-2 for more details in which it illustrates the effect of lateral heat transfer during first thermal time.

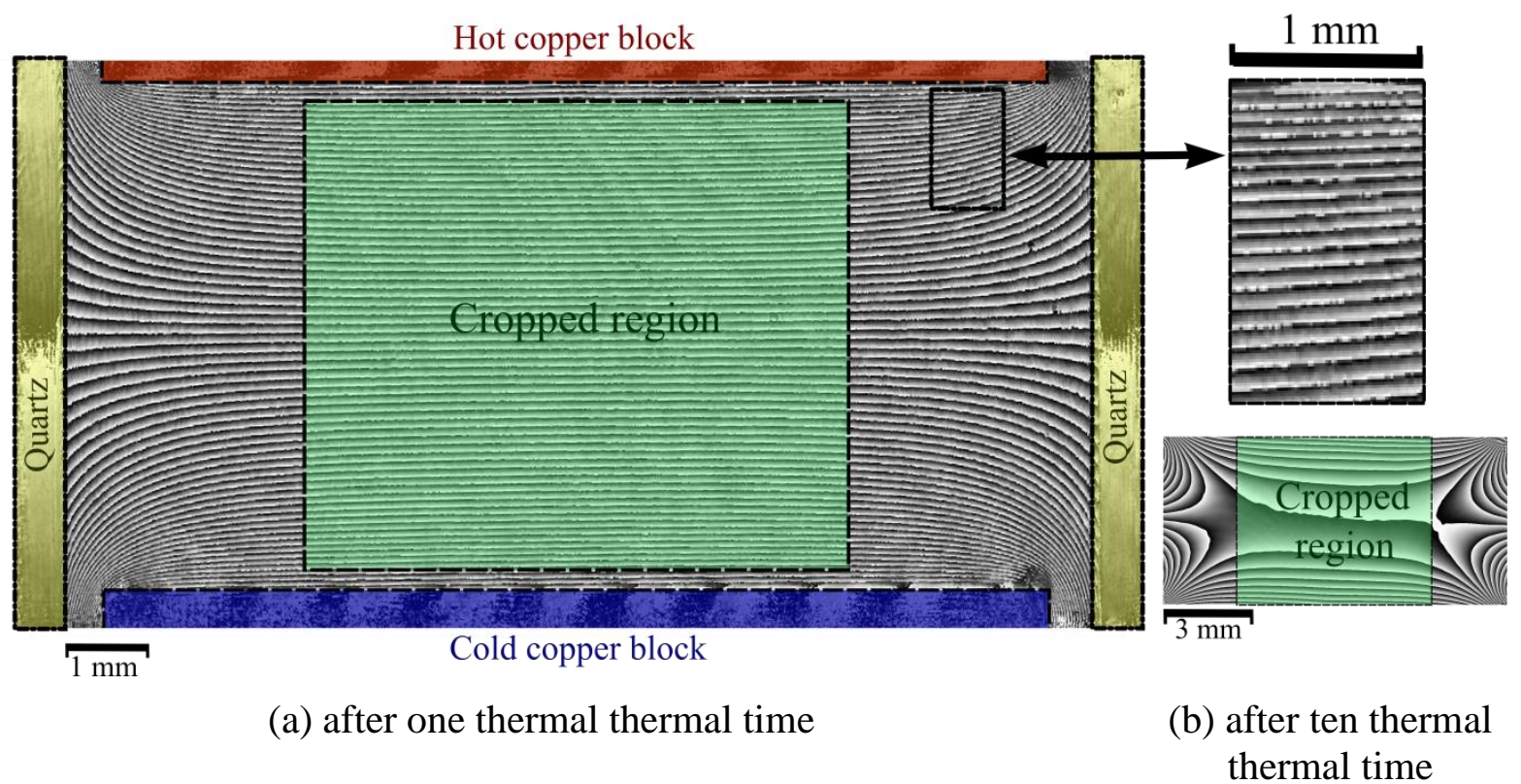

Figure 9-2: General phase maps demonstrating the front view of the cell and also the cropped region of the cell

In addition to cropping from the lateral sides of the image, 90 to $95 \%$ of the height of the cell at the center of the cell is analyzed. It is because there is a stronger temperature gradient at the boundaries; while, having a linear temperature gradient is essential to obtain a pure linear variation of concentration. Only in this condition, the Soret coefficient can be measured 
accurately. Furthermore, the reflection of the glass and copper block caused wiggly fringes close to the boundaries.

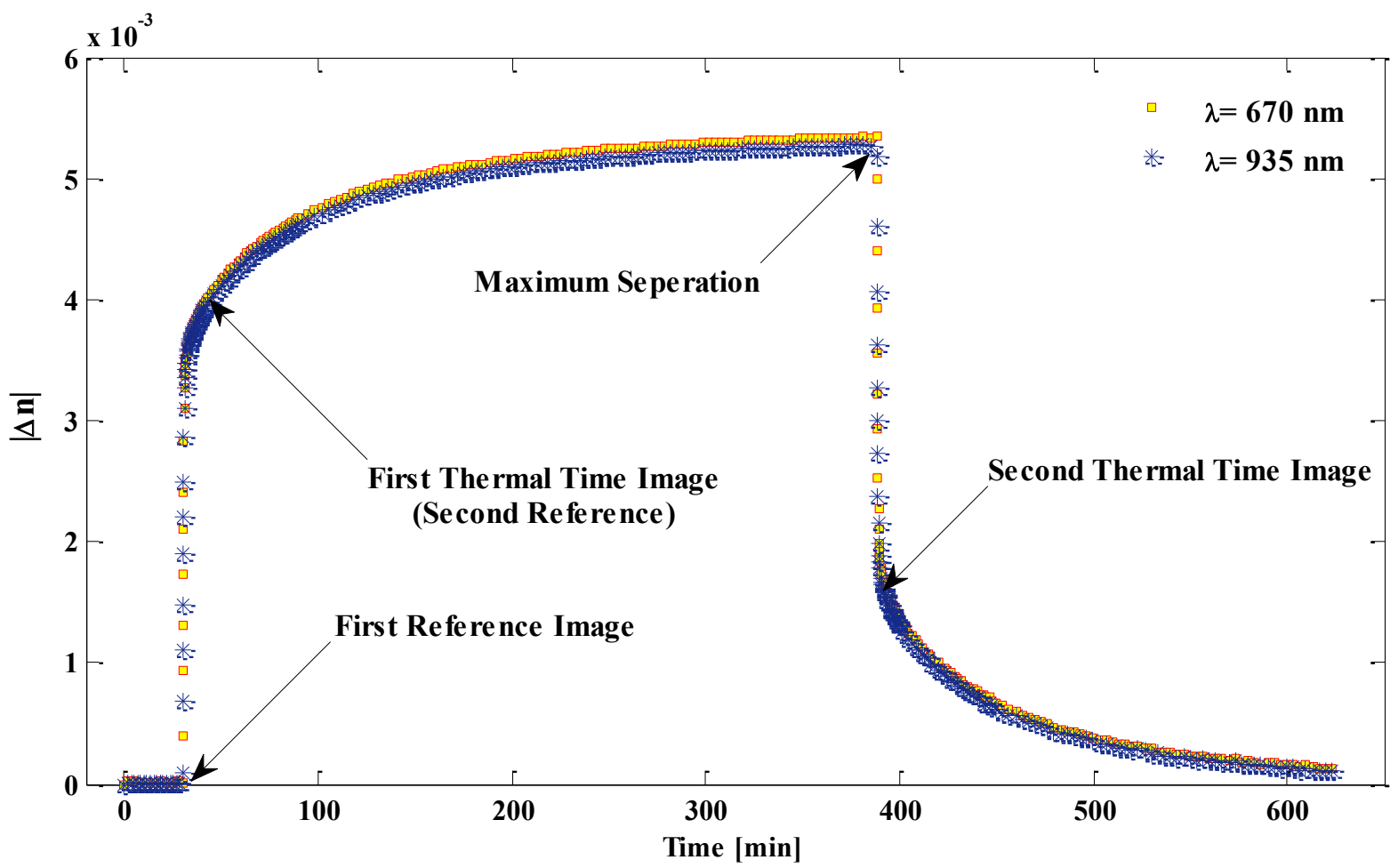

Figure 9-3: Refractive index difference between cell walls during the entire experiment for cell 4

Here, the fringe pattern can be seen inside the liquid region and the glass parts. In the magnified part of Figure 9-2, the stronger temperature gradient close to the cold and hot copper block is evident. While the bigger figure map (part a) shows the phase distribution after one thermal time; the small figure map (part c at the right and bottom) illustrates the subtracted phase map between the reference image (the image which is taken right after thermal time) and phase image taken after ten thermal times. The impact of heat transfer from the lateral side is evident in this subplot and based on heat losses the green region must be cropped and processed for the entire image to measure the concentration variation caused by the Soret effect.

\subsubsection{Refractive Index Measurement}

Figure 9-3 shows the refractive index difference between the hot and cold walls for an experimental case of cell 4 with $\Delta \mathrm{T}=10 \mathrm{~K}$. While the refractive index measured using both lasers shows similar behavior, slight differences exist in their results. For instance, the value of $\Delta \mathrm{n}$ 
measured by laser with the wavelength of $670 \mathrm{~nm}$ always gives a higher value. This can be observed in Figure 9-3 sorely.
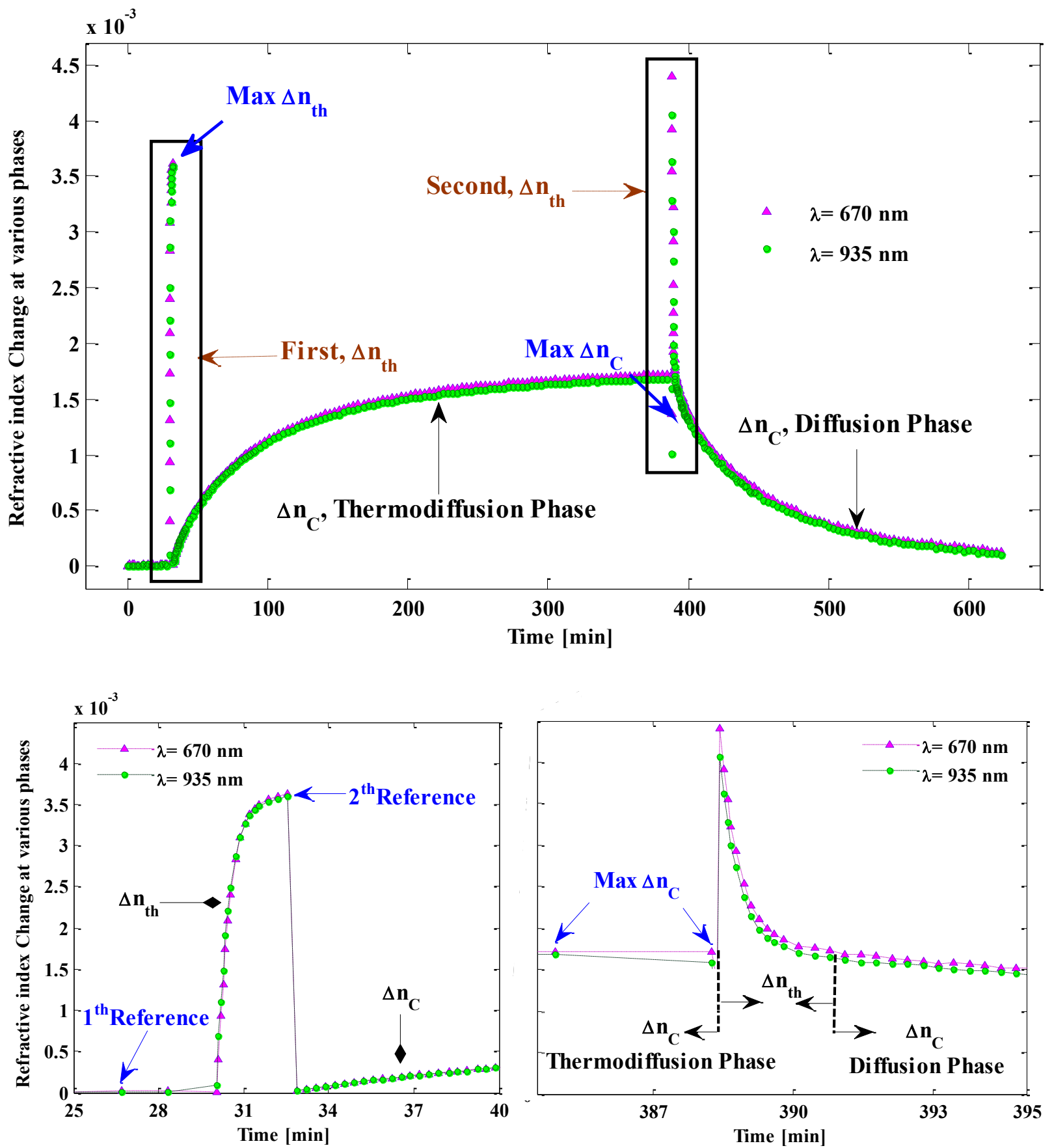

Figure 9-4: Refractive index difference during five different steps of the experiment which can be used for measurement of the concentration and temperature and experimental error depending on various periods. 
Using this small different behaviors of the lasers along with the slight variation of contrast factors between these two lasers, the separation in the ternary system for all three components can be extracted. It is worthy to note that sometimes these small variations of the laser behaviors and contrast factors of the mixture cause sensitive measurement of the Soret coefficient for the cases with small amount of separation $\left(>4 \times 10^{-4}\right)$.

In order to obtain the change of refractive index caused by change of temperature and concentration, a reference image was selected before application of the thermal gradient and then it is subtracted from all images. In other words, subtracting a reference image removes the error caused by various sources other than temperature or concentrating variation. To separate the changes due to temperature variation from the concentration variation the second reference image was chosen at the end of thermal time (180 s). At the end of the thermodiffusion phase, the maximum refractive index difference between walls can be seen owing to the maximum separation in the domain. By removing the temperature gradient after six hours, another thermal time must be considered for the system. All of these pointes (or images) are demonstrated in Figure 9-3. After subtracting the first and second references from the result, Figure 9-4 is obtained.

According to Figure 9-4, the experiment duration can be divided into five phaes. First is the duration between the first reference and applying temperature gradient ( $\mathrm{t}=25$ to $30 \mathrm{~min}$ ). Result of this step ideally should be zero for the entire points in the domain; however, this result represents the experimental error of the refractive index measurement using MZI. After applying temperature gradient and until the first thermal time is second phase ( $\mathrm{t}=30$ to $33 \mathrm{~min}$ ). The results here were used for temperature measurement in the domain. The results between the first thermal time and the second one demonstrate the concentration change ( $t=33$ to $388 \mathrm{~min}$ ), then there is a phase for second thermal time $(\mathrm{t}=388$ to $391 \mathrm{~min})$.

Eventually, the fifth phase would be diffusion phase where there is no temperature gradient. It can be seen that the variation during the first phase is at least three orders of magnitude smaller than temperature measurement value (phases 2 and 4) and two orders of magnitude smaller than thermodiffusion and diffusion steps (phases 3 and 5). This proves the accuracy of the measurement on-board ISS. 


\subsubsection{Linear Variation of Temperature along the Height of the Cell}

A linear variation of the temperature along the height of the cell is an essential condition that should be examined for the cropped area. In addition, it is vital to consider starting the separation as soon as a linear temperature gradient is achieved between the two walls. Theoretically, the separation starts when temperature gradient applies. However, since the thermodiffusion process is very slow and based on the principles of MZI for measurement of coupled heat and mass transfer, this is the best possible time to be considered for starting of the thermodiffusion phase.

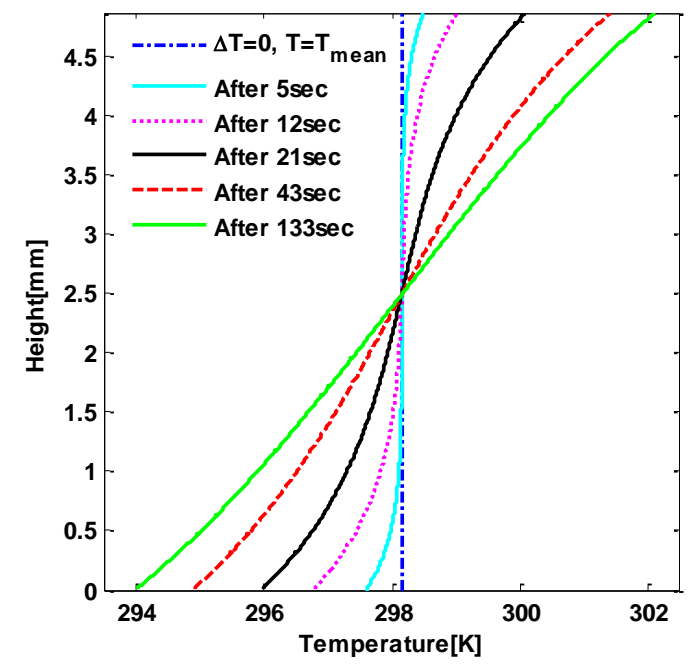

(a) laser $\lambda=670 \mathrm{~nm}$

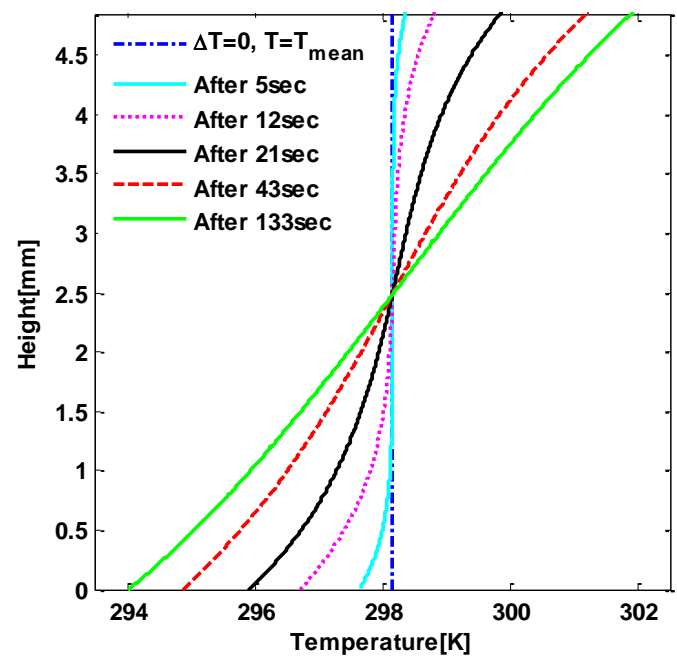

(b) laser $\lambda=935 \mathrm{~nm}$

Figure 9-5: Temperature along the height of the cell

Figure 9-5 shows the temperature variation along the height of the cell measured by both laser. While both lasers represent identical measurement of the temperature, due to considering $90 \%$ of the height of the cell lower temperature gradient than $10 \mathrm{~K}$ is recorded. Nevertheless, the most important fact is the linear variation of the temperature inside the cropped area that can be seen in these plots (green lines).

It can be realized that the thermal time for this experiment would not exceed more than $150 \mathrm{~s}$ or at most 3 minutes. Nonetheless, it can be observed that both lasers provided similar temperature variation results versus time at the middle of the cell with less than a $0.07 \mathrm{~K}$ difference. This proves the high accuracy of the thermal part of the apparatus. It must be mentioned that the 
maximum experimental error in the temperature measurement is less than $0.04 \mathrm{~K}$ for both wavelengths.

\subsubsection{Temporal Concentration Variation during Thermodiffusion and Diffusing phases}

The maximum concentration difference of two components can be measured using the results of Figure 9-4. The separation results of IBB and THN for cell 4 with $\Delta \mathrm{T}=10 \mathrm{~K}$ are plotted in parts Figure 9-6 a and b, respectively.

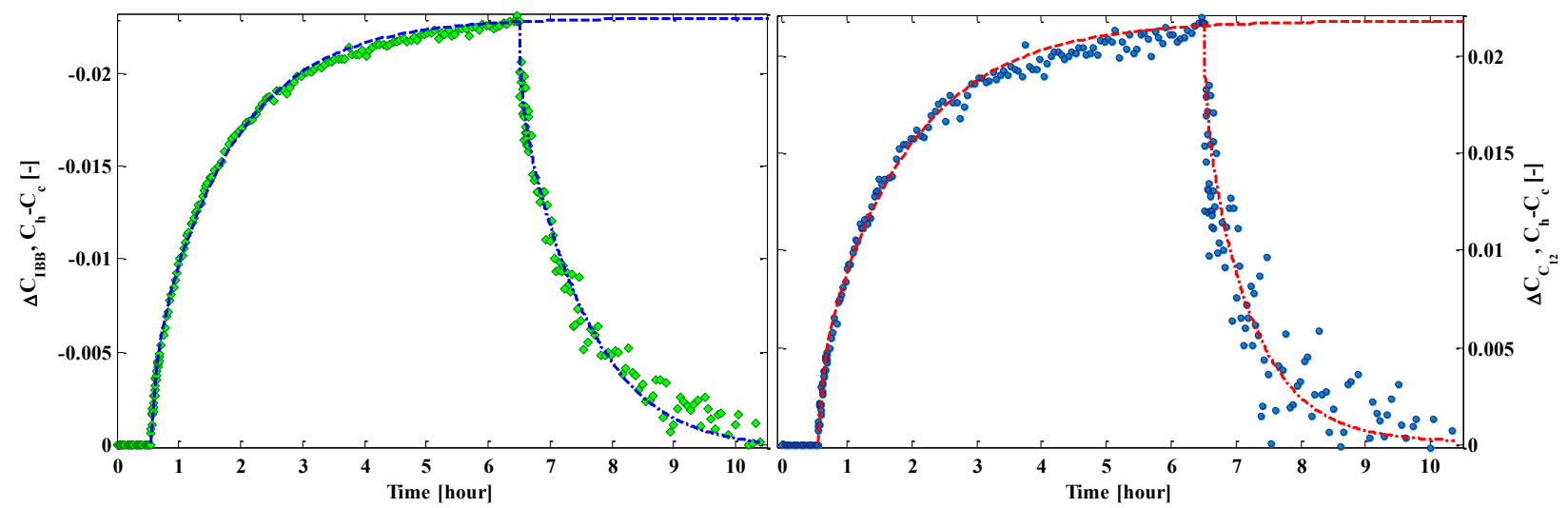

(a) $\mathrm{C}_{12}$

(b) IBB

Figure 9-6: Concentration difference between the hot and cold sides for two of the components versus time during thermodiffusion and diffusion phases for cell 4

It is evident that THN has smoothly separated toward the cold side of the cell versus time. It results in a positive Soret effect and is consistent for most of the cases that is discussed in detail in the next section. There was similar separation for IBB, although it moved toward the hot wall and showed a negative Soret effect for all cases. The separation magnitude of both THN and IBB were similar, with the separation of THN being slightly stronger. This means that the third component separated toward the hot side weakly.

Despite this weaker separation of $\mathrm{C}_{12}$, which can be barely detected for cells 4 and $5, \mathrm{C}_{12}$ shows a negative thermodiffusion coefficient in this mixture at a mean temperature of $298 \mathrm{~K}$ (compare maximum separation value of THN and $\mathrm{C}_{12}$ in Figure 9-6 and Figure 9-7), the same observation has been reported for cell 2 in the previous study [27]. 


\subsubsection{Soret and Diffusion Coefficients Measurement using GA Curve Fitting Method}

Equations 9-4 and 9-5 can be used to estimate $\mathrm{S}_{\mathrm{T}}$ and $\mathrm{D}$ simultaneously by implementing the GA. For the ternary mixture, there are four free parameters during the thermodiffusion phase (two Soret coefficients and two diffusion coefficients) and also four free parameters during diffusion phase (two Soret coefficients and two diffusion coefficients) to be evaluated at each iteration of solving the curve-fitting problem.

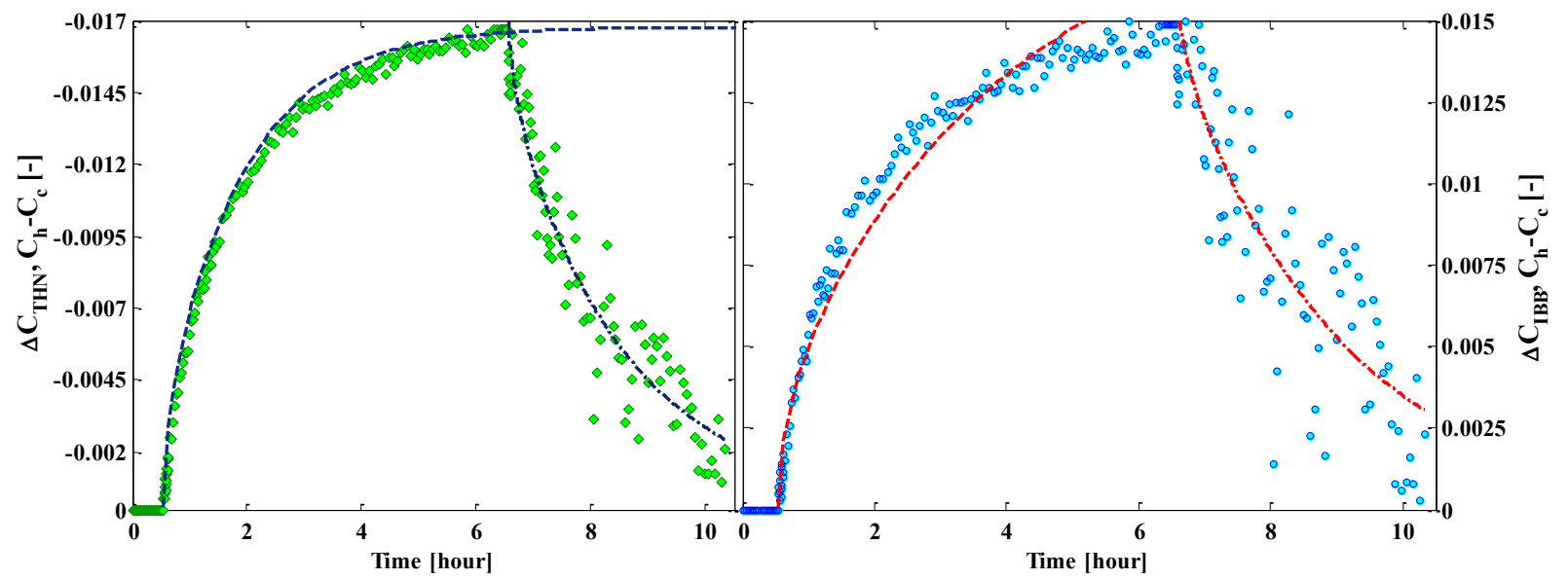

(a)THN

(b)IBB

Figure 9-7: Concentration difference between the hot and cold side for two of the components versus time during thermodiffusion and diffusion phases for cell 3

The following objective function is used for this purpose;

$$
\text { Err }=\sum_{i=1}^{n}\left[\frac{1}{n}\left(t+\tau_{D}\right)\left(C_{\exp }-C_{\text {curve }}\right)^{2}\right]
$$

where $\mathrm{n}$ denotes the number of images, and $\mathrm{t}$ represents the time used to intensify the influence of each data point according to the time at which the data is recorded and eventually, $\mathrm{C}_{\mathrm{exp}}$ and $\mathrm{C}_{\text {curve }}$ are the experimental concentration and the current fitted curve, respectively. While, Figure 9-6 and Figure 9-7 demonstrate the separation of THN and IBB for cell 4 and with $\Delta T=10$ K during thermodiffusion and diffusion phases; Figure 9-8 shows the concentration difference of all three components between the hot and cold sides during thermodiffusion phases for cell 2. 


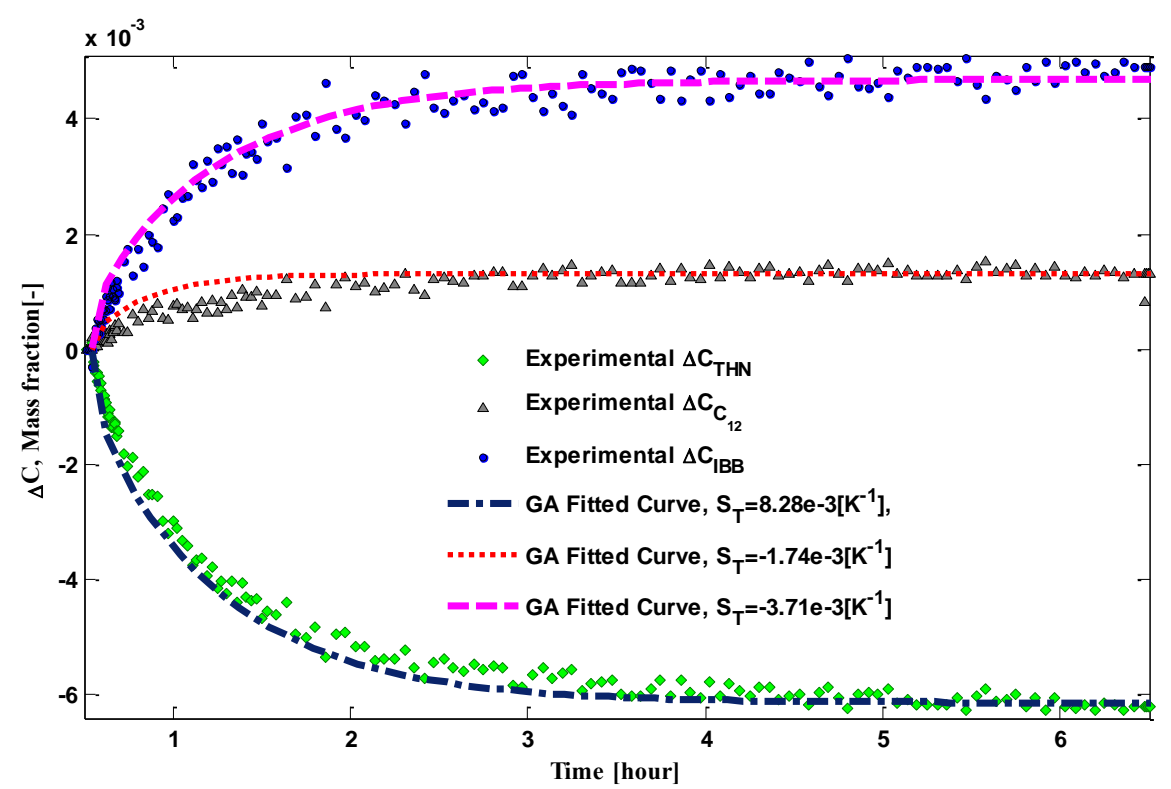

Figure 9-8: Concentration difference of all three components between the hot and cold sides during thermodiffusion phases for cell 2

The result of curve fitting procedure is also plotted in these figures. Comparing these results with temporal experimental data was performed showing good match with experimental trends especially for THN and IBB. According to Figure 9-8, after four to five hours, steady-state separation was achieved so there is no need of using GA fitting curve; however, Figure 9-6 and Figure 9-7 show the noticeable slope of the diagram representing more separation at the end of thermodiffusion time. Thus, in general, using a fitting method for estimation of various diffusion coefficients is essential for this specific problem.

\subsubsection{Separation of Non-associating Ternary Mixture along the Temperature Difference}

Based on best of our knowledge, this is the first time that Soret coefficients of this mixture with these specific compositions have been measured (except for composition of cell 2). In addition to that, this is a unique study that took advantage of the reduced gravity environment to measure Soret coefficient for ternary non-associating mixtures. It can be seen in Figure 9-9, compositions are chosen for this series of experiments systematically to present the behavior of Soret separation mostly in the regions that the summation of the initial concentration of one or two components include more than 80 percent of the final compositions. This means the central region in Figure 9-9 must be analyzed separately. The numbers in this figure illustrate the 
assigned cell number. We can claim that the cases identified with red number should mostly compare together and then with cases with green number.

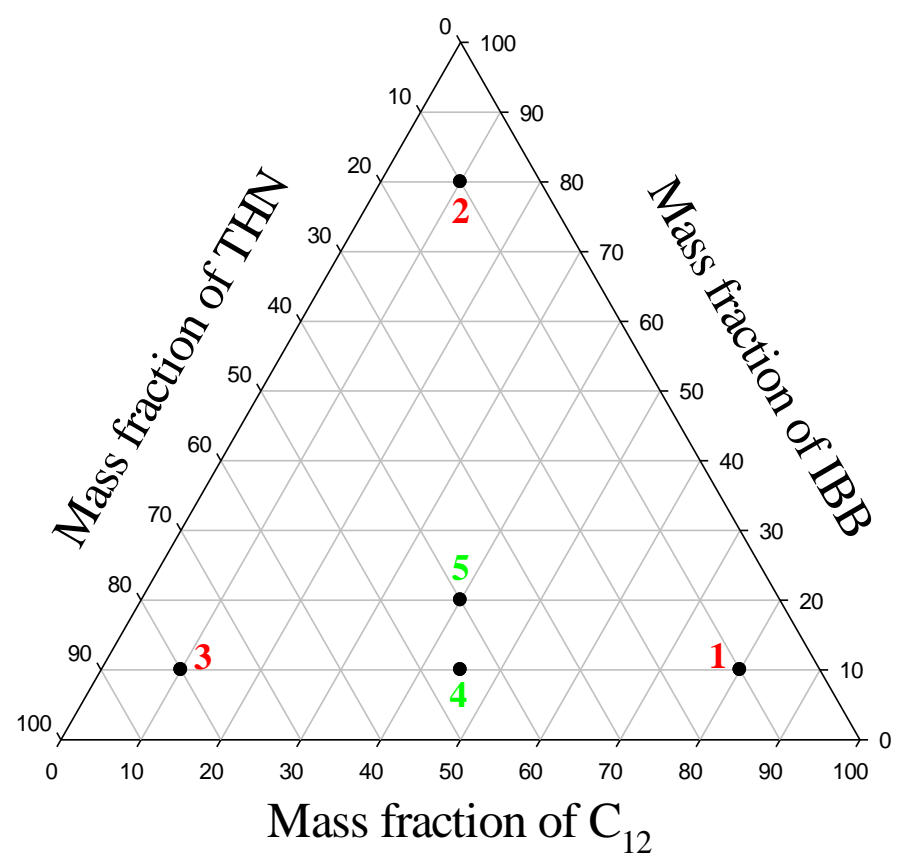

Figure 9-9: Ternary map of mass fraction of ternary mixtures tested onboard ISS, numbers represent the cell number

As mentioned before, the measurement of concentration (and not temperature) for any ternary system is highly sensitive to measurement of the concentration contrast factors. This fact is not true for binary mixture; since the mathematical procedure for calculation of the concentration is the same as temperature measurement for binary mixture. For binary mixtures, error in the contrast factor measurement has direct effect on measurement of temperature and concentration; while, for ternary system two coupled equations should be solved using the contrast factors. In this condition, only 10 percent inaccuracy of the measurement of concentration contrast factors may drastically affect the final results. This inaccuracy may cause up to 100 percent variation in Soret coefficients measurement. Thus, nowadays more and more various precise optical methods are being developed to measure contrast factors. On the other hand, there is no doubt in accuracy of refractive index measurement inside the cells using MZI owing to the advance image processing technique that we used here (WFT) and high precision of the MZI results. Consequently, in addition to maximum $\Delta \mathrm{T}, \Delta \mathrm{C}_{\mathrm{THN}}, \Delta \mathrm{C}_{\mathrm{IBB}}, \Delta \mathrm{C}_{\mathrm{C} 12}$ and Soret coefficients of the 
components, we have reported the maximum $\Delta \mathrm{n}_{\text {th }}$ and $\Delta \mathrm{n}_{\mathrm{C}}$ for both lasers, this data points are demonstrated in Figure 9-4.

Table 18: Detail experimental results of thermodiffusion of the ternary mixture onboard ISS

\begin{tabular}{|c|c|c|c|c|c|c|c|c|c|c|}
\hline $\begin{array}{c}\text { Case } \\
\#\end{array}$ & $\begin{array}{c}\text { Cell } \\
\#\end{array}$ & $\begin{array}{c}\mathrm{T}_{\text {mean }} \\
{[\mathrm{C}]}\end{array}$ & $\begin{array}{c}\text { Cropped } \\
\text { height } \\
{[\mathrm{mm}]}\end{array}$ & $\begin{array}{c}\lambda_{1}=670 \mathrm{~nm} \\
\operatorname{Max} \\
\Delta \mathrm{n}_{\mathrm{T}} \times 10^{3}\end{array}$ & $\begin{array}{c}\lambda_{1}=670 \mathrm{~nm} \\
\operatorname{Max} \\
\Delta \mathrm{n}_{\mathrm{C}} \times 10^{4}\end{array}$ & $\begin{array}{c}\lambda_{2}=935 \mathrm{~nm} \\
\operatorname{Max} \\
\Delta \mathrm{n}_{\mathrm{T}} \times 10^{3}\end{array}$ & $\begin{array}{c}\lambda_{2}=935 \mathrm{~nm} \\
\operatorname{Max} \\
\Delta \mathrm{n}_{\mathrm{C}} \times 10^{4}\end{array}$ & $\begin{array}{c}\Delta \mathrm{C}_{\mathrm{THN}} \\
\times 10^{3} \\
{[-]}\end{array}$ & $\begin{array}{c}\Delta \mathrm{C}_{\mathrm{IBB}} \\
\times 10^{3} \\
{[-]}\end{array}$ & $\begin{array}{c}\Delta \mathrm{C}_{\mathrm{C} 12} \\
\times 10^{3}[-]\end{array}$ \\
\hline 1 & \multirow{4}{*}{1} & 25 & 4.53 & 3.54 & 5.10 & 3.50 & 4.93 & 6.29 & -0.30 & $\begin{array}{l}-5.99 \\
\end{array}$ \\
\hline 2 & & 25 & 4.49 & 3.53 & 5.12 & 3.51 & 4.92 & 6.63 & -0.53 & -6.10 \\
\hline 3 & & 40 & 4.48 & 3.41 & 4.54 & 3.37 & 4.39 & 5.29 & -0.28 & -5.01 \\
\hline 4 & & 40 & 4.43 & 3.32 & 4.55 & 3.30 & 4.40 & 5.86 & -0.90 & -4.96 \\
\hline 5 & \multirow{4}{*}{2} & 25 & 4.59 & 3.80 & 4.32 & 3.74 & 4.19 & 6.84 & -5.57 & -1.27 \\
\hline 6 & & 25 & 4.73 & 4.16 & 4.53 & 4.10 & 4.38 & 6.22 & -4.73 & -1.49 \\
\hline 7 & & 40 & 4.80 & 4.24 & 4.10 & 4.22 & 3.93 & 6.76 & -5.26 & -1.50 \\
\hline 8 & & 40 & 4.63 & 4.04 & 4.04 & 3.99 & 3.87 & 6.81 & -5.36 & -1.45 \\
\hline 9 & \multirow{4}{*}{3} & 25 & 4.68 & 3.97 & 10.99 & 3.93 & 10.69 & 19.40 & -17.30 & -2.10 \\
\hline 10 & & 25 & 4.44 & 3.75 & 9.15 & 3.69 & 8.90 & 16.49 & -14.36 & -2.13 \\
\hline 11 & & 40 & 4.42 & 3.83 & 9.77 & 3.75 & 9.49 & 17.20 & -15.70 & -1.50 \\
\hline 12 & & 40 & 4.59 & 3.97 & 9.66 & 3.91 & 9.50 & 20.60 & -18.74 & -1.86 \\
\hline 13 & \multirow{4}{*}{4} & 25 & 4.49 & 3.98 & 18.42 & 3.95 & 17.96 & -1.10 & 22.80 & -21.70 \\
\hline 14 & & 40 & 4.43 & 3.47 & 16.87 & 3.44 & 16.38 & -1.73 & 22.72 & -20.99 \\
\hline 15 & & 40 & 4.73 & 3.99 & 16.59 & 3.95 & 16.11 & 1.76 & 18.93 & -20.69 \\
\hline 16 & & 40 & 4.59 & 3.91 & 16.39 & 3.87 & 15.91 & 1.95 & 18.45 & -20.40 \\
\hline 17 & \multirow{4}{*}{5} & 25 & 4.59 & 3.85 & 16.94 & 3.84 & 16.38 & 2.00 & 22.20 & -24.20 \\
\hline 18 & & 25 & 4.59 & 3.84 & 16.56 & 3.82 & 16.13 & 0.30 & 22.70 & -23.00 \\
\hline 19 & & 40 & 4.63 & 4.11 & 13.06 & 4.10 & 12.64 & 1.14 & 16.88 & -18.02 \\
\hline 20 & & 40 & 4.79 & 4.15 & 14.80 & 4.13 & 14.33 & 1.36 & 20.20 & -21.56 \\
\hline
\end{tabular}

These four values along with contrast factors can be used to calculate the maximum concentration and temperature difference between the cold and hot walls. Thus for the case of more accurate measurement of contrast factors in future, the Soret measurement of these ternary mixture can be easily adjusted. In this study the contrast factor measured by Galand[95] has been used. Eventually, all of the aforementioned parameters are listed in Table 18 for all cases. It should be noted that the experiment has been repeated more than four times at each specific condition to ensure the repeatability of the experimental results. Here, we reported the results of twenty cases presenting ten different conditions. All the measured temperature gradients were below the assigned temperature $(\Delta \mathrm{T}=10 \mathrm{~K})$, it is mainly because of cropping the fringe domain and considering about $4.5 \mathrm{~mm}$ of the height. The considered height of the cell is also showed in 
Table 18. One can see that the results of the similar cases are very close that proves the repeatability of the experiment results.

As indicated earlier, comparing the results of first three cells proves a good understating of the separation in multicomponent mixture, when the majority of the cell is filled with only one of the components. The first observation for these cases is that the magnitude of the separation for two components is noticeably greater than the third one. It may represent that two components mostly two of the three components have separated and third one has moved toward the hot or cold wall strongly.

Table 19: Mass transport coefficients of the ternary system measured in microgravity environment

\begin{tabular}{|c|c|c|c|c|c|c|c|c|c|c|}
\hline $\begin{array}{c}\text { Cell } \\
\#\end{array}$ & THN & IBB & $\begin{array}{c}\mathrm{C} 1 \\
2 \\
\end{array}$ & $\begin{array}{c}\text { Tme } \\
\text { an }\end{array}$ & $\begin{array}{c}\mathrm{ST}, \\
\mathrm{THN} \times 103\end{array}$ & $\begin{array}{c}\mathrm{ST}, \\
\mathrm{IBB} \times 103\end{array}$ & $\begin{array}{c}\mathrm{ST}, \\
\mathrm{C} 12 \times 103\end{array}$ & $\begin{array}{c}\text { DTHN } \times 1 \\
010\end{array}$ & $\begin{array}{c}\text { DIBB } \\
\times 1010 \\
\end{array}$ & $\begin{array}{r}\text { DC12 } \\
\times 1010 \\
\end{array}$ \\
\hline 1 & 10 & 10 & 80 & 25 & $8.33 \pm 0.4$ & $-0.42 \pm 0.2$ & $-4.37 \pm 0.2$ & \multirow{2}{*}{$6.72 \pm 0.6$} & \multirow{2}{*}{$1.98 \pm 0.6$} & \multirow{2}{*}{$9.86 \pm 0.6$} \\
\hline 1 & 10 & 10 & 80 & 40 & $7.68 \pm 0.4$ & $-0.82 \pm 0.3$ & $-3.86 \pm 0.3$ & & & \\
\hline 2 & 10 & 80 & 10 & 25 & $8.57 \pm 0.6$ & $-4.85 \pm 0.5$ & $-1.78 \pm 0.4$ & \multirow{2}{*}{$6.71 \pm 0.6$} & \multirow{2}{*}{$5.89 \pm 0.6$} & \multirow{2}{*}{$2.17 \pm 0.6$} \\
\hline 2 & 10 & 80 & 10 & 40 & $8.43 \pm 0.3$ & $-3.74 \pm 0.2$ & $-1.83 \pm 0.2$ & & & \\
\hline 3 & 80 & 10 & 10 & 25 & $12.78 \pm 0.9$ & $-20.12 \pm 1.2$ & $-2.76 \pm 0.6$ & \multirow{2}{*}{$3.91 \pm 0.6$} & \multirow{2}{*}{$1.93 \pm 0.6$} & \multirow{2}{*}{$\begin{array}{c}0.968 \pm 0 \\
6\end{array}$} \\
\hline 3 & 80 & 10 & 10 & 40 & $15.07 \pm 1.1$ & $-24.11 \pm 1.9$ & $-2.37 \pm 0.5$ & & & \\
\hline 4 & 45 & 10 & 45 & 25 & $-0.71 \pm 0.6$ & $30.3 \pm 0.4$ & $-10.32 \pm 0.2$ & \multirow{2}{*}{$1.345 \pm 0.6$} & \multirow{2}{*}{$5.22 \pm 0.6$} & \multirow{2}{*}{$5.47 \pm 0.6$} \\
\hline 4 & 45 & 10 & 45 & 40 & $0.88 \pm 0.2$ & $24.01 \pm 0.3$ & $-9.57 \pm 0.2$ & & & \\
\hline 5 & 40 & 20 & 40 & 25 & $0.64 \pm 0.4$ & $16.27 \pm 0.3$ & $-11.45 \pm 0.4$ & \multirow{2}{*}{$5.59 \pm 0.6$} & \multirow{2}{*}{$4.8 \pm 0.6$} & \multirow{2}{*}{$1.06 \pm 0.6$} \\
\hline 5 & 40 & 20 & 40 & 40 & $0.61 \pm 0.3$ & $11.78 \pm 0.3$ & $-9.86 \pm 0.5$ & & & \\
\hline
\end{tabular}

The second fact can be express as follows: the component with maximum initial mass fraction is always one of the components that has separated strongly. In all cases, THN separated toward the cold side of the cell versus time, which is characteristic of a positive Soret effect. On the other hand, IBB and $\mathrm{C}_{12}$ always present negative Soret as they moved toward the hot sides. The magnitude of separation for cells 1 and 2 are similar and remarkably lower than cell 3 that contained $80 \%$ THN. It can be interpreted as strong tendency of the THN to separate when the temperature gradient is applied to the mixture. In cell 1 that has maximum mass fraction of $\mathrm{C}_{12}$; IBB showed minimum separation and for cell 2 , which contained $80 \%$ IBB, $\mathrm{C}_{12}$ showed minimum separation. While, results of cell 3 can be used to compare the tendency of separation of IBB versus $\mathrm{C}_{12}$; because both of these components have same minor initial mass fractions of $10 \%$. In addition to that, both of IBB and $\mathrm{C}_{12}$ showed negative Soret effect in the result of cells 1 and 2. The results of case 3 show that IBB and THN have been separated strongly. The 
molecular mass of THN and IBB are close; however, the heavier IBB, experienced a separation toward the hot side and THN, which has an intermediate molecular mass, strongly separated toward the cold wall. Finally, it can be concluded that the lighter component moved toward the cold side. The compositions of the ternary mixture for cell 4 and 5 are similar as mentioned by green number in Figure 9-9, consequently the final separation results for both case are similar as well; while, it can be seen that by decreasing the initial mass fraction of IBB, the separation of the components is more pronounced. A critical observation here is small THN separation toward the hot side, in contrast IBB has moved toward the cold side. THN always has separated toward the cold wall except for cell at mean temperature of $298 \mathrm{~K}$ in which THN weakly separated toward the hot side. This observation somehow can be explained using the fact that the molecular mass of THN and IBB are close. Consequently, because of this similarity in their physical properties, they swapped their role in separation of cell 4 and 5, in which IBB migrated toward the cold wall and THN experienced an intensive weak separation. Nevertheless, $\mathrm{C}_{12}$ always shows positive Soret coefficient.

\subsubsection{Mass Transport Coefficients of THN, IBB and $\mathrm{C}_{12}$ Mixtures}

According to the results that are provided in Figure 9-6, Figure 9-7 and Figure 9-8 and using curve-fitting method, Soret coefficient of the components were measured and reported in Table 19. It should be noted that the values of curve-fitting method using genetic algorithm showed a

slightly greater value of $S_{\mathrm{T}}$ comparing to the Soret coefficient that is calculated using steady state equation. This means that although the steady-state condition is visible after 6 to 8 hours of thermodiffusion phase; there still is a small ratio of separation of the components in the system (especially for result of cell 3 that have smaller values for D).

Two last columns of Table 19 represent the pure diffusion coefficients of the ternary mixture and not cross diffusion coefficient. The diffusion coefficients in Table 19 show smaller value of D for the component that experienced a weak separation. In other words, it can be paraphrased as follows: the separation of the component with minimum $\Delta \mathrm{C}$ started later than separation of other components and has a slower speed of separation. In addition, one can see weaker mass diffusion coefficient for IBB in comparison with IBB and THN. By comparing the mass diffusion coefficients of different mixtures, it is evident that cell 3 with maximum initial mass fraction of 
$80 \%$ THN has the lowest values for D. In contrast, cell 2 that contained $80 \% \mathrm{C}_{12}$ has greater values of $\mathrm{D}$. This fact is also observable by comparing Figure 9-6 and Figure 9-7.

\subsubsection{Temperature Dependence on Soret Coefficient}

The Soret coefficients of these mixtures were determined in the experimental mean temperature of $298.15 \mathrm{~K}$ and $313.15 \mathrm{~K}$ by means of Mach Zehnder Interferometry. It can be seen that changing mean temperature from $298.15 \mathrm{~K}$ to $313.15 \mathrm{~K}$ does not have a noticeable impact on the Soret coefficients. However, few differences can be observed when the mean temperature is changed. For instance, the sign of the Soret coefficients for all components has not changed except for the mixture in cell 4 (mass fractions of $0.45,0.10$, and 0.45 with respect to THN, IBB and $\mathrm{C}_{12}$ ). In this case the separation of THN was weak. In addition to that, while the impact of change of mean temperature on Soret coefficient of THN and $\mathrm{C}_{12}$ is marginal, Soret Coefficients of IBB showed a stronger dependency to the mean temperature.

\subsection{Conclusion}

The results of space experiment (DSC experiment that was proposed to analyze the Soret effect in the non-associating ternary mixture) are reported in this study. Here, the Soret coefficients and pure molecular diffusion coefficients of THN-IBB-C12 mixture for five different initial mass fractions have been determined. In order to ensure the quality and repeatability of the experiment for each individual case, two repeated cases are provided and processed. The Windowed Fourier transform image processing aided with a curve-fitting method and GA were employed to obtain the mass transfer coefficients.

The linear variation of the temperature along the height of the cells has been proved for the cropped (or analyzed) region of the fringes. It was shown that six hours of thermodiffusion time are sufficient to reach steady-state separation of two cells and other needed more time to get to that condition. Requiring more time for separation means smaller value of mass diffusion coefficient, which is observed, for the case with $80 \%$ initial mass fraction of IBB. On the other hand, cell 1 with $80 \%$ mass fraction of $\mathrm{C}_{12}$ showed a very fast diffusion process. The values of refractive index measurement at the end of each step of the experiment are reported, so the Soret coefficient can be re-calculated according to any other measurement of contrast factors. 
The separation results provoked that heavier component (C12) always moved toward the hot side and THN with an intermediate molecular mass separated toward the cold wall for most of the cases (except for cell 4 at mean temperature of 298 K). Since the molecular mass of THN and IBB are close, IBB in some occasions experienced the separation toward the hot side and in other cases; it went through the cold sides (Cell 4 and 5). In the condition that IBB moved toward the cold side the separation of THN was relatively weak and close to zero.

In this ternary mixture, it is observed that the component that filled the majority of the cell has separated strongly. In all cases, the magnitude of the separation of two components was similar and noticeably higher than the third component. Finally, the impact of mean temperature of the Soret coefficient of the mixture has been analyzed. We observed no noticeable change of Soret and diffusion coefficient due to change of mean temperature from $298.15 \mathrm{~K}$ to $313.15 \mathrm{~K}$ for these non-associating hydrocarbon mixtures. Nevertheless, it should be noted that only for THN at the condition of low separation and IBB, a slight change of Soret coefficients was detected. 


\section{Chapter 10: Conclusions, and Future Works}

In this thesis, the Soret diffusion coefficients and diffusion coefficients of two binary mixtures and five ternary mixtures have been measured. In order to conduct this measurement, an advance image processing technique has been developed. New experimental models were proposed to measure the experimental thermal time for the MZI experiment and to measure the diffusion coefficients of ternary mixture.

\subsection{Thesis Contributions}

The contributions of this research can be summarized as followings:

1) Enhancement of the 2-D Fourier and 2-D phase unwrapping analysis for interferometry experiments to study thermodiffusion phenomenon.

2) Implementation of the windowed Fourier transform method, Genetic algorithm and fast robust smoothing function to analyze the optical digital interferometry experiments that deals with heat and mass transfer thermodiffusion interferometry experiments for the first time. It has a robust methodology to calculate mass transfer coefficients. The developed software incorporates analytical solutions and CFD code of this problem.

3) Development of an application for processing the MZI results for windows and MAC users along with a graphical user interface (GUI). While similar applications can be found, this is the first application, which is specified for analyzing the heat and mass transfer in binary or ternary mixtures.

4) Design and development of a robust methodology to estimate, calculate and measure the accurate thermal time for the MZI experiments. In this method, the EA method and fast robust smoothing method are used for the first time to analyze the results of the Soret experiment.

5) Design and development of a robust methodology to estimate, calculate and measure the Soret and diffusion coefficients for binary and ternary mixtures based on MZI experiments. 
6) Visualizing and measuring the impacts of the force vibration on the mass transfer due to Soret effect. Since the SODI apparatus provide two different views of the cell, the temperature and concentration profiles inside the cell can be obtained for two perpendicular plates (views). This means the ability to visualize the convection cells and the flow pattern in the cell caused by external vibrations.

7) Detailed heat and mass transfer analysis for the two series of the experiments on-board international space station (ISS) under SODI facilities and measurement of the Soret and diffusion coefficients of the binary and ternary mixtures.

\subsection{Thesis Conclusions}

\subsubsection{Development of a Model to Study the Thermodiffusion using MZI}

A new formulation to calculate the thermal relaxation time for the Soret effect experiment by means of Mach-Zehnder interferometry was proposed for the negative Soret effect, and an experimental approach was provided for measurement in the case of the positive Soret effect. For both negative and positive Soret effects, it was demonstrated that the precise thermal time for MZI must not exceed more than $40 \%$ of $\mathrm{L}^{2} / \chi$. It was proved that for a thermal time equal to $\mathrm{L}^{2} /$ $\chi$, about $10 \%$ underestimation in the measurement of the Soret is observed. We also introduced an iterative optimization method to correct the final values of the Soret coefficient and the maximum separation when $\mathrm{L}^{2} / \chi$ is assumed as the thermal time for MZI thermodiffusion experiments.

A new experimental method to measure the pure and cross diffusion coefficients of any transparent liquid mixture using Mach-Zehnder interferometry is proposed. The essential postprocessing procedure that was required to extract the diffusion matrix from the fringe images is provided in detail. The Soret phenomenon was used as the driving force to initiate the concentration variation at the beginning of the diffusion process. The analytical solution of the diffusion mass transfer in a ternary system along with an optimization method using an evolutionary algorithm was employed to estimate the values of the diffusion matrix. The purpose of this technique was examined versus the experimental results obtained on-board ISS. Finally, 
the advantages and limitations of this technique were discussed and the essential recommendations were made to minimize the influences of the limitations that were pointed out.

The application of the two-dimensional Windowed Fourier Transform (WFT) for thermodiffusion interferometry experiments were investigated for the first time. It is shown that if WFT parameters are properly chosen, it can significantly reduce the wiggles from the fringe pattern of an MZI thermodiffusion experiment. The FFT method gives a reliable measurement of temperature for such a problem when a large thermal gradient $(\sim 5 \mathrm{~K})$ exists between the cold and hot walls and when it is not essential to record precisely the temperature variations at different points in the domain. Results show that the WFT noticeably improves the measurement of concentration. This improvement is more evident when dealing with very small variations in ternary systems. More precisely, the FFT fails to extract a reasonable concentration profile for the ternary system. It is concluded that when the maximum separation in the domain is less than 0.003 [wt], results of the FFT method are not reliable and may introduce more than $40 \%$ error in the measurements.

\subsubsection{Heat Transfer Analysis of the MZI Experiment for Space Experiment}

The sensitivity of the temperature measurement using MZI for these experiments is approximately $10^{-3} \mathrm{~K}$ according to the current image processing techniques. This means that stronger fluctuations in temperature profile were not triggered by optical or post-processing errors. Despite relatively inferior performance of the IVIDIL thermal controller unit at $\Delta \mathrm{T}=0$, temperature fields inside cell 2 showed a uniform temperature field after the application of the temperature gradient (consisting of temperature variations of less than $0.02 \mathrm{~K}$ ). The optical temperature measurement proved the sensitivity of the controller unit before applying the temperature gradient; however, it also showed the accuracy of unit when $\Delta \mathrm{T}>0$. When $\Delta \mathrm{T}=0$, the temperature variation at the middle of cell 1 causes a high temperature gradient close to the walls; however, at the central region of the cell, the variation of the temperature was less than $0.05 \mathrm{~K}$. For cell 2 (in the DSC experiment), the combination of the thermal sensor and controller unit was more accurate than in case 1 for the measurement with the larger distance between the thermal sensors. The heat flux close to the lateral walls of the DSC cell was stronger than that the 
IVIDIL case, because in addition to insufficient insulation, a linear variation of heat fluxes from cold wall to hot wall was not observed.

\subsubsection{Experimental Study on Impacts of Thermogravitational Flow on Heat and Mass Transfer}

The steady temperature difference between the hot and cold sides, measured optically, differed between $6 \%$ and $11 \%$ from the value reported by the thermal controller unit. Moreover, it was demonstrated that the fringe pattern close to the lateral walls of cell 2 should be cropped before processing the results. The measured $\mathrm{S}_{\mathrm{T}}$ using the optical temperature results provides a better agreement with the benchmark value and the previously known optical beam deflection results within a $3 \%$ deviation; however, using the thermal sensor temperature difference provided about $10 \%$ deviation from the benchmark value.

The impacts of various selective forced vibration levels and fixed temperature gradients on the measurement of thermodiffusion are determined. The results were compared with the analytical trends in the convection-free regime without any forced or g-jitter vibration using benchmark values of diffusion coefficients. Results revealed maximum separation for the case with the minimum Rayleigh number; however, a linear relationship between vibrational Rayleigh number and maximum separation was not found. The influence of vibrations on flow patterns caused completely different separation profiles at high Gershuni numbers. For Gs higher than a critical value, the effect of an increase in the vibration level decreased, and a linear decrement in separation was detected. While most of the runs reached a steady-state condition before the end of diffusion time, the run with the smallest Gs required more time to reach this condition. Finally, it was shown that the ISS provides a suitable environment for this experiment, and the results of the IVIDIL experiments are consistent and can be used as a reference to validate experiments under the Earth gravity existence conditions.

\subsubsection{Measurement of the Mass transfer Coefficients of Binary and Ternary Mixtures}

The Soret coefficients and pure molecular diffusion coefficients of THN-IBB-C12 mixture at five different initial mass fractions have been determined. In order to ensure the quality and 
repeatability of the experiment for each individual case two repeated cases are provided and processed.

The linear variation of the temperature along the height of the cells has been observed for the cropped (or analyzed) region of the fringes. It was shown that six hours of thermodiffusion time is sufficient to reach steady-state separation of two cells while others needed more time to get to the same condition. Requiring longer time for separation means smaller value of mass diffusion coefficient, which is observed for the case with $80 \%$ initial mass fraction of IBB. On the other hand, cell 1 with $80 \%$ mass fraction of $\mathrm{C} 12$ showed a very fast diffusion process. The values of refractive index measurement at the end of each step of the experiment are reported, so the Soret coefficient can be re-calculated according to any other measurement of contrast factors.

The separation results provoked that heavier component (C12) always moved toward the hot side and THN with an intermediate molecular mass separated toward the cold wall for most of the cases (except for cell 4 at mean temperature of $298 \mathrm{~K}$ ). Since the molecular mass of THN and IBB are close, IBB in some occasions experienced the separation toward the hot side and in others, it went through the cold sides (Cell 4 and 5). In the condition that IBB moved toward the cold side the separation of THN was relatively weak and close to zero.

In this ternary mixture, it is observed that the component that filled the majority of the cell has separated strongly. In all cases, the magnitude of the separation of two components was similar and noticeably higher than the third component. Finally, the impact of mean temperature of the Soret coefficient of the mixture has been analyzed. We observed no noticeable change of Soret and diffusion coefficients due to change of mean temperature from $298.15 \mathrm{~K}$ to $313.15 \mathrm{~K}$ for this non-associating hydrocarbon mixtures. Nevertheless, it should be noted that only for THN at the condition of low separation and IBB a slight change of Soret coefficients has been detected.

\subsection{Future Work}

While in this study, for the first time the mass transport coefficients of a ternary are measured using Mach Zehnder interferometry setup in reduced gravity environment; this experiment must be repeated for other non-associating and associating ternary mixtures to have enough 
experimental data. Having these experimental results guides to develop a theoretical model, this fairly predicts the mass transfer coefficient for binary and ternary mixtures.

Many numerical analyses have studied the impact of force vibration on the heat and mass transfer in the presence of the Soret effect; however, no direct comparison between the experimental results in the rescued gravity environment and the direct numerical simulation of the problem has been conducted.

The only available model to study the impact of force vibration on thermodiffusion experiment is introduced by Gershuni. He proposed a non-dimensional number based on Rayleigh number, which represents the strength of the induced convection caused by temperature gradient and oscillatory vibration (thermogravitational flow). Nonetheless, this number is not function of any mass transfer properties of the mixture and it cannot implicitly represent the impact of force vibration on the mass transfer due to the Soret effect. Consequently, a new model must be developed that describes the impact of the oscillatory vibration/acceleration on mass transfer due to thermodiffusion phenomenon.

Numerous studies have claimed the optical impact of glass is one of the main sources of uncertainly in the MZI experiment; while, this effect must be determined accurately with a firm model that can be applied for other experimental cases.

In this work along with many other studies, the uncertainties of this optical experiment have been addressed in general. While no specific work is dedicated to the uncertainties of the MZI experiment for the thermodiffusion experiment. 


\section{Appendix A: Publications}

\section{Accepted journal papers:}

1-Ahadi, Amirhossein, Saghir, M. Z. Theoretical and Experimental Determination of Thermal Time Using Mach-Zehnder Interferometry to Study Heat and Mass Transfer Phenomena, Journal of Applied Thermal Engnieering (2014). IF=2.47

2-Khoshnevis, Ahmad, Ahadi, Amirhossein, \& Saghir, M. Z. On the influence of prevailing residual accelerations and disturbances on board International Space Station on diffusion process in liquids. International Journal of Applied thermal engineering, (2014). IF=2.12

3- Ahadi, Amirhossein, Saghir, M. Z. New Experimental Method to Measure Pure and Cross Diffusion Coefficients of Transparent Ternary Mixtures Using Mach-Zehnder Interferometry, Optics and Lasers in Engineering (2014). IF=2.12

4-Ahadi, Amirhossein, Giraudet, C., Jawad, H., Croccolo, F., Bataller, H., \& Saghir, M. Z. Experimental, Theoretical and numerical investigation of Solute separation for a binary hydrocarbon mixture in porous medium. International Journal of Thermal Sciences (2014). $\mathrm{IF}=2.47$

5-Ahadi, Amirhossein, \& Saghir, M. Z. An Extensive Heat Transfer Analysis using Mach Zehnder Interferometry during Thermodiffusion Experiment on board the International Space Station. International Journal of Applied thermal engineering, (2013). IF=2.12

6-Ahadi, Amirhossein, \& Saghir, M. Z. Experimental Study of the Impacts of Forced Vibration on Thermodiffusion Phenomenon in Microgravity Environment. International Journal of Applied thermal engineering, (2013). $\mathrm{IF}=2.12$

7-Ahadi, Amirhossein, Varenbergh, S. Van, \& Saghir, M. Z. Measurement of Soret Coefficients for a Ternary Hydrocarbon Mixture in Low Gravity Environment. J.Chem.Phys, (2013), IF=3.33

8-Ahadi, Amirhossein, Khoshnevis, A., \& Saghir, M. Z. Windowed Fourier Transform as an Essential Digital Interferometry Tool to Study Coupled Heat and Mass Transfer. Optics \& Laser Technology, (2013). IF=1.58

9- Ahadi, Amirhossein, Kianian, A., \& Saghir, M. Z. Heat and mass transport phenomena under influence of vibration using a new aided image processing approach (2013). International Journal of Thermal Sciences. IF=2.47

10- Ahadi, Amirhossein, \& Saghir, M. Z. Transient effect of micro vibration from two space vehicles on mixture during thermodiffusion experiment (2013). Microgravity Science and Technology. IF=0.67 
11-Khoshnevis, A., Ahadi, Amirhossein, \& Saghir, M. Z. Influence of static and oscillatory gravity fields on thermodiffusion of a binary mixture. (2013) International Journal of Thermal Sciences, $1-12$. IF $=2.47$

12-Kianian, A., Ahadi, Amirhossein, \& Saghir, M. Z. Experimental evidence of low Rayleigh vibration on mixture during thermodiffusion experiment(2012). The Canadian Journal of Chemical Engineering. IF=1.00

13- Ahadi, Amirhossein, Yousefi, T., \& Saghir, M. Z. Double diffusive convection and thermodiffusion of fullerene-toluenenanofluid in a porous cavity(2013). The Canadian Journal of Chemical Engineering, IF=1.00

14- Ahadi, Amirhossein, \& Saghir, M. Z. Quasi steady state effect of micro vibration from two space vehicles on mixture during thermodiffusion experiment(2012). FDMP; Fluid Dynamics and Material Processing, 287. $\mathrm{IF}=0.87$

\section{Submitted Journal Paper:}

15- Ahadi, Amirhossein, Varenbergh, S. Van, Galand, Q., \& Saghir, M. Z., Soret and Diffusion Coefficients Measurement of Ternary Mixtures of Dodecane, Isobutylbenzene and 1,2,3,4Tetrahydronaphthalene on-board International Space Station. J.Chem.Phys

\section{Conference Proceedings}

1-Ahadi, Amirhossein, Q. Galand, S. V. Varenbergh and M. Z. Saghir (2013). Soret Diffusion Measurement of the Benchmark Mixtures onboard ISS. 64th Internationa IAC conference, beijing China.

2- Ahadi, Amirhossein, Khoshnevis, A., Dejmek, M., \& Saghir, M. Z. (2012). Influence of Low and High Frequrncy G-jitter Vibrations on Mixture During Thermodiffsuion Experiment. CASI ASTRO in Quebec City April 23rd - 26th Quebec City (p. 12).

3- Khoshnevis, A., Ahadi, Amirhossein, Dejmek, M., \& Saghir, M. Z. (2012). Sensitivity of Gjitter Vibrations on Thermodiffusion Experiment onboard International Space Station. CASI ASTRO in Quebec City April 23rd - 26th Quebec City.

4-Kianian, A., Saghir, M. Z., \& Ahadi, Amirhossein. (2012). Experimental Evidence of Low Rayleigh Vibrations on Mixture During Thermodiffsuion Experiment. CASI ASTRO in Quebec City April 23rd - 26th Quebec City (p. 33) 


\section{References}

[1] Z. Liu, S.-L. Chen, X. Ge, P. Dong, J. Gao, Z. Xu, Measurement of Diffusion Coefficient of Heavy Oil in Fluidized Catalytic Cracking (FCC) Catalysts, Energy \& Fuels. 24 (2010) $2825-2829$.

[2] S. Hartmann, W. Kohler, K.I. Morozov, The isotope Soret effect in molecular liquids: a quantum effect at room temperatures, Soft Matter. 8 (2012) 1355-1360.

[3] S. Srinivasan, M.Z. Saghir, Significant Equation of State and Viscosity on the Thermodiffusion Coeffcients of a Ternary Hydrocarbon Mixture., High Temp. High Press. 39 (2010) 65-81.

[4] A. Ahadi, C. Giraudet, H. Jawad, F. Croccolo, H. Bataller, M.Z. Saghir, Experimental, Theoretical and numerical investigation of Solute separation for a binary hydrocarbon mixture in porous medium, Int. J. Therm. Sci. (n.d.).

[5] M. Mojtabi, Y. Razi, K. Maliwan, A. Mojtabi, Influence of vibrations on Soret-driven convection in porous media, Numer. Heat Transf. Part A Appl. 46 (2004) 981-993.

[6] A. Mahidjiba, M. Mamou, P. Vasseur, Onset of double-diffusive convection in a rectangular porous cavity subject to mixed boundary conditions, 43 (2000) 1505-1522.

[7] M.Z. Saghir, C.G. Jiang, M. Chacha, Y. Yan, M. Khawaja, S. Pan, Transport Phenomena in Porous Media III, Elsevier, 2005.

[8] K. Ghorayeb, A. Firoozabadi, Ghorayeb, K. and Firoozabadi , A., 2000, "Modeling multicomponent diffusion and convection in porous media, Soc. Pet. Eng. J. 5 (2000) 158.

[9] K. Ghorayeb, A. Firoozabadi, Interpretation of the Unusual Fluid Distribution in the Yufutsu Gas-Condensate Field, SPE Journal,Reservoir Eng. Res. Inst.; Toshiyuki Anraku, Japan Pet. Explor. Co. 8 (2003) 114-123.

[10] K. Ghorayeb, T. Anraku, A. Firoozabadi, Interpretation of the fluid distribution and GOR behavior in the Yufutsu fractured gas-condensate field, in: SPE Asia Pacific Conf. Integr. Model. Asset Manag., 2000: pp. 1-10.

[11] J.R. Petit, J. Jouzel, D. Raynaud, N.I. Barkov, J.-M. Barnola, I. Basile, et al., Climate and atmospheric history of the past 420,000 years from the Vostok ice core, Antarctica, Nature. 399 (1999) 429-436.

[12] R. Piazza, Thermophoresis: moving particles with thermal gradients, Soft Matter. 4 (2008) 1740-1744. 
[13] R. Piazza, A. Parola, Thermophoresis in colloidal suspensions, J. Phys. Condens. Matter. 20 (2008) 153102.

[14] D. Vigolo, R. Rusconi, H.A. Stone, R. Piazza, Thermophoresis: microfluidics characterization and separation, Soft Matter. 6 (2010) 3489-3493.

[15] S. Duhr, D. Braun, Optothermal Molecule Trap, arXiv Prepr. Physics/0606121. 97 (2006) 38103.

[16] S. Duhr, D. Braun, Optothermal Molecule Trapping by Opposing Fluid Flow with Thermophoretic Drift, Phys. Rev. Lett. 97 (2006) 38103.

[17] S. Duhr, D. Braun, Why molecules move along a temperature gradient, Proc. Natl. Acad. Sci. (2011) 19678-19682,103(53).

[18] K.J. Zhang, M.E. Briggs, R.W. Gammon, J. V. Sengers, Optical measurement of the Soret coefficient and the diffusion coefficient of liquid mixtures, J. Chem. Phys. 104 (1996) 6881-6892.

[19] J.K. Platten, M.M. Bou-Ali, J.F. Dutrieux, Precise determination of the Soret, thermodiffusion and isothermal diffusion coefficients of binary mixtures of dodecane, isobutylbenzene and 1,2,3,4-tetrahydronaphthalene (contribution of the University of Mons to the benchmark test), Philos. Mag. 83 (2003) 2001-2010.

[20] M. Eslamian, M.Z. Saghir, Dynamic thermodiffusion model for binary liquid mixtures., Phys. Rev. E. Stat. Nonlin. Soft Matter Phys. 80 (2009) 011201.

[21] A. Kianian, A. Ahadi, M.Z. Saghir, Experimental evidence of low Rayleigh vibration on mixture during thermodiffusion experiment, in: Can. J. Chem. Eng., 2012: pp. 1-12.

[22] J.K. Platten, M.M. Bou-Ali, P. Costeseque, J.F. Dutrieux, W. Kohler, C. Leppla, et al., Benchmark values for the Soret, thermal diffusion and diffusion coefficients of three binary organic liquid mixtures, 83 (2003).

[23] G. Wittko, W. Koehler, Precise determination of the Soret, thermal diffusion and mass diffusion coefficients of binary mixtures of dodecane, isobutylbenzene and 1,2,3,4tetrahydronaphthalene by a holographic grating technique, Philos. Mag. 83 (2003) 19731987.

[24] J.F. Dutrieux, J.K. Platten, G. Chavepeyer, M.M. Bou-Ali, D.J. F., P.J. K., et al., On the Measurement of Positive Soret Coefficients, J. Phys. Chem. B. 106 (2002) 11.

[25] P. Blanco, M.M. Bou-Ali, J.K. Platten, D.A. de Mezquia, J.A. Madariaga, C. Santamaria, Thermodiffusion coefficients of binary and ternary hydrocarbon mixtures, J. Chem. Phys. 132 (2010) 114506. 
[26] A. Koniger, H. Wunderlich, W. Koehler, Measurement of diffusion and thermal diffusion in ternary fluid mixtures using a two-color optical beam deflection technique, J. Chem. Phys. 132 (2010) 174506.

[27] A. Ahadi, S. Van Varenbergh, M.Z. Saghir, Measurement of the Soret coefficients for a ternary hydrocarbon mixture in low gravity environment., J. Chem. Phys. 138 (2013) 204201.

[28] M. Eslamian, M.Z. Saghir, M.M. Bou-Ali, Investigation of the Soret effect in binary, ternary and quaternary hydrocarbon mixtures: New expressions for thermodiffusion factors in quaternary mixtures, Int. J. Therm. Sci. 49 (2010) 2128-2137.

[29] S. Sharidan, N. Amin, I. Pop, G-jitter fully developed combined heat and mass transfer by mixed convection flow in a vertical channel, Int. Commun. Heat Mass Transf. 32 (2005) $657-665$.

[30] R. Savino, R. Monti, Convection induced by residual-g and g-jitters in diffusion experiments, Int. J. Heat Mass Transf. 42 (1999) 111-126.

[31] Y. Yan, K. Jules, M.Z. Saghir, A comparative study of G-jitter effect on thermal diffusion board the International Space Station., Fluid Dyn. Mater. Process. J. 3 (3) (2008) 231-245.

[32] Y. Yan, V. Shevtsova, M.Z. Saghir, Numerical study of low frequency g-jitter effect on thermal diffusion, FDMP Fluid Dyn. Mater. Process. 1 (2005) 315-328.

[33] Y. Yan, S. Pan, K. Jules, M.Z. Saghir, Vibrational effect on thermal diffusion under different microgravity environments., Microgravity Sci. Technol. 19b (2007) 12-25.

[34] H.P. A. Ryskin, H.-W. Muller, A. Ryskin, H. Pleiner, Thermodiffusion effects in convection of ferrofluids, MAGNETOHYDRODYNAMICS. 39 (2003) 51-55.

[35] S. Srinivasan, M.Z. Saghir, Experimental approaches to study thermodiffusion - A review, Int. J. Therm. Sci. 50 (2011) 1125-1137.

[36] S. Srinivasan, M.Z. Saghir, Measurements on thermodiffusion in ternary hydrocarbon mixtures at high pressure, J. Chem. Phys. 131 (2009) 124508.

[37] S. Srinivasan, M. Dejmek, M.Z. Saghir, Thermo-solutal-diffusion in high pressure liquid mixtures in the presence of micro-vibrations, Int. J. Therm. Sci. 49 (2010) 1613.

[38] C.G. Jiang, T.J. Jaber, H. Battaler, Z.M. Saghir, Simulation of Ludwig-Soret effect of a water-ethanol mixture in a cavity filled with aluminum oxide powder under high pressure, Int. J. Therm. Sci. 47 (2008) 126-135. 
[39] M. Touzet, G. Galliero, V. Lazzeri, M.Z. Saghir, F. Montel, J.-C. Legros, Thermodiffusion: From microgravity experiments to the initial state of petroleum reservoirs, Comptes Rendus Mcaniquecanique. 339 (2011) 318-323.

[40] O. Thomas, Reservoir analysis based on copositional gradients, stanford university, 2007.

[41] A. Ahadi, T. Yousefi, M.Z. Saghir, Double diffusive convection and thermodiffusion of fullerene-toluenenanofluid in a porous cavity, Can. J. Chem. Eng. 9999 (2013) 12.

[42] R. Piazza, "Thermal forces": colloids in temperature gradients, J. Phys. Condens. Matter. 16 (2004) S4195.

[43] F. Winkel, S. Messlinger, W. Schepf, I. Rehberg, M. Siebenbarger, M. Ballauff, Thermal convection in a thermosensitive colloidal suspension, New J. Phys. 12 (2010) 53003.

[44] R. Monti, R. Savino, M. Lappa, On the convective disturbances induced by g-jitter on the space station, Acta Astronaut. 48 (2001) 603-615.

[45] R. Savino, R. Monti, M. Piccirillo, Thermovibrational Convection in a Fluid Cell, Comput. Fluids. 27 (1998) 923.

[46] R. Monti, R. Savino, A new approach to g-level tolerability for fluid and material science experiments, Acta Astronaut. 37 (1995) 313-331.

[47] M. Eslamian, M.Z. Saghir, G. Jiang, On the Role of the Velocity Frame of Reference and Alternative Definitions of the Soret Coefficients in Thermodiffusion, Review. (n.d.).

[48] M. Eslamian, M.Z. Saghir, Microscopic study and modeling of thermodiffusion in binary associating mixtures, Phys. Rev. 80 (2009) 61201.

[49] S. Srinivasan, M. Eslamian, M.. Saghir, Estimation of the thermodiffusion coefficients for n-Dodecane/n-Butane/Methane mixtures and comparison with experimental data from Foton M3 mission., IAC. (IAC-09-A2 (2009).

[50] S. VanVaerenbergh, S. Srinivasan, M.Z. Saghir, Thermodiffusion in multicomponent hydrocarbon mixtures: Experimental investigations and computational analysis, J. Chem. Phys. 131 (2009) 114505.

[51] J.F. Torres, A. Komiya, E. Shoji, J. Okajima, S. Maruyama, Development of phaseshifting interferometry for measurement of isothermal diffusion coefficients in binary solutions, Opt. Lasers Eng. 50 (2012) 1287-1296.

[52] X. Zhang, N. Hirota, T. Narita, J.P. Gong, Y. Osada, K. Chen, Investigation of Molecular Diffusion in Hydrogel by Electronic Speckle Pattern Interferometry, J. Phys. Chem. B. 103 (1999) 6069-6074. 
[53] A. Cadavid Orozco, J. Garzón, Optical Method For Liquid Diffusional Coefficients Calculation, Rev. Colomb. Física. 43 (2011) 507.

[54] M. Eslamian, M.Z. Saghir, A Critical Review of Thermodiffusion Models: Role and Significance of the Heat of Transport and the Activation Energy of Viscous Flow, J. NonEquilibrium Thermodyn. 34 (2009) 97-131.

[55] M. Eslamian, M.Z. Saghir, Dynamic Thermodiffusion Model for Binary Liquid Mixture, Phys. Rev. E 80. (2009) 11201.

[56] A. Abbasi, M.Z. Saghir, M. Kawaji, Evaluation of the activation energy of viscous flow for the binary mixture in order to estimat the thermodiffusion coefficient, J. NonEquilibrium Thermodyn. (2008).

[57] A. Abbasi, M.Z. Saghir, M. Kawaji, Study of thermodiffusion of carbon dioxide in binary mixtures of $\mathrm{n}$-butane $\&$ carbon dioxide and $\mathrm{n}$-dodecane $\&$ carbon dioxide in porous media, Int. J. Therm. Sci. 50 (2011) 124-132.

[58] A. Abbasi, M.Z. Saghir, M. Kawaji, A new proposed approach to estimate the thermodiffusion coefficients for linear chain hydrocarbon binary mixtures, J.Chem.Phys. 131 (2009) 14502.

[59] E.L.D. Jr., H.G. Drickamer, Thermal diffusion and molecular motion in liquids, Jounral Phys. Chem. 59 (n.d.) 443.

[60] Y. Demirel, S.I. Sandler, Linear-nonequilibrium thermodynamics theory for coupled heat and mass transport, Int.J.Heat Mass Transf. 44 (2001) 2439.

[61] B.-C. Tai, M.-I. Char, Soret and Dufour effects on free convection flow of non-Newtonian fluids along a vertical plate embedded in a porous medium with thermal radiation, Int. Commun. Heat Mass Transf. 37 (2010) 480.

[62] S. Srinivasan, M.Z. Saghir, Impact of the vibrations on Soret separation in binary and ternary mixtures, Fluid Dyn. Mater. Process. 7 (2011) 201-216.

[63] A. Ahadi, M.Z. Saghir, Transient effect of micro vibration from two space vehicles on mixture during thermodiffusion experiment, Microgravity Sci. Technol. 25 (2013) 127139.

[64] A. Ahadi, M.Z. Saghir, Quasi steady state effect of micro vibration from two space vehicles on mixture during thermodiffusion experiment, FDMP; Fluid Dyn. Mater. Process. 8 (2012) 397-422.

[65] V. Shevtsova, IVIDIL experiment onboard the ISS, Adv. Sp. Res. 46 (2010) 672-679. 
[66] V. Shevtsova, T. Lyubimova, Z. Saghir, D. Melnikov, Y. Gaponenko, V. Sechenyh, et al., IVIDIL: on-board g-jitters and diffusion controlled phenomena, Int. Symp. Phys. Sci. Sp. 327 (2011) 12031.

[67] V. Shevtsova, D. Melnikov, A. Mialdun, J. Legros, Development of convection in binary mixture with soret effect, Microgravity Sci. Technol. 18 (2006) 38-41.

[68] A. Kianian, A. Ahadi, M.Z. Saghir, Experimental evidence of low rayleigh vibration on mixture during thermodiffusion experiment, Can. J. Chem. Eng. 91 (2013) 1568-1574.

[69] M. Chacha, D. Faruque, M.Z. Saghir, J.C. Legros, Solutal thermodiffusion in binary mixture in the presence of g-jitter, Int. J. Therm. Sci. 41 (2002) 899-911.

[70] A. Parsa, S. Srinivasan, M.Z. Saghir, Impact of density gradients on the fluid flow inside a vibrating cavity subjected to soret effect, Can. J. Chem. Eng. 91 (2012) 550-559.

[71] P.A.L. Narayana, P. Sibanda, Soret and Dufour effects on free convection along a vertical wavy surface in a fluid saturated Darcy porous medium, Int. J. Heat Mass Transf. 53 (2010) 3030-3034.

[72] V.K. Chhaniwal, A. Anand, C.S. Narayanamurthy, <title>Diffusion coefficient measurement of transparent liquid solutions using digital holographic interferometry</title>, in: W. Osten, C. Gorecki, E.L. Novak (Eds.), Opt. Metrol., International Society for Optics and Photonics, 2005: pp. 1009-1013.

[73] A. Parsa, M.Z. Saghir, Thermodiffusion in a binary fluid mixture subject to external vibrations: effect of variable physical properties, in: ASME/JSME 2011 8th Therm. Eng. Jt. Conf., ASME, Honolulu, Hawaii, USA, March 13-17, 2011: pp. T10024-T10024-5.

[74] H. Nasrabadi, H. Hoteit, A. Firoozabadi, An analysis of species separation in a thermogravitational column filled with a porous medium, Transp. Porous Media. 67 (2007) 473-486.

[75] A. Mialdun, V. Yasnou, V. Shevtsova, A. Königer, W. Köhler, D. Alonso de Mezquia, et al., A comprehensive study of diffusion, thermodiffusion, and Soret coefficients of waterisopropanol mixtures, J. Chem. Phys. 136 (2012) 244512.

[76] On thermal diffusion and convection in multicomponent mixtures with application to the thermogravitational column, Phys. Fluids. 19 (2007) 27101.

[77] J.K. Platten, M.M. Bou-Ali, J.F. Dutrieux, Enhanced Molecular Separation in Inclined Thermogravitational Columns, J. Phys. Chem. B. 107 (2003) 11763-11767. 
[78] M. Marucci, S.-G. Pettersson, G. Ragnarsson, A. Axelsson, Determination of a diffusion coefficient in a membrane by electronic speckle pattern interferometry: a new method and a temperature sensitivity study, J. Phys. D. Appl. Phys. 40 (2007) 2870.

[79] Determination of calibration function in thermal field flow fractionation under thermal field programming, J. Sep. Sci. (2006).

[80] F. Croccolo, H. Bataller, F. Scheffold, A light scattering study of non equilibrium fluctuations in liquid mixtures to measure the Soret and mass diffusion coefficient., J. Chem. Phys. 137 (2012) 234202.

[81] Y. Kishikawa, S. Wiegand, R. Kita, Temperature Dependence of Soret Coefficient in Aqueous and Nonaqueous Solutions of Pullulan, Biomacromolecules. 11 (2010) 740-747.

[82] W. Koehler, Thermodiffusion in polymer solutions as observed by forced Rayleigh scattering, J. Chem. Phys. 98 (1993) 660-668.

[83] C. Leppla, S. Wiegand, Investigation of the Soret effect in binary liquid mixtures by thermal-diffusion-forced Rayleigh scattering (contribution to the benchmark test), Philos. Mag. 83 (2003) 1989-1999.

[84] Sign change of the Soret coefficient of poly(ethylene oxide) in water/ethanol mixtures observed by thermal diffusion forced Rayleigh scattering, J. Chem. Phys. 121 (2004) 3874 .

[85] W. Koehler, A. Koniger, B. Meier, Measurement of the Soret, diffusion, and thermal diffusion coefficients of three binary organic benchmark mixtures and of ethanol water mixtures using a beam deflection technique, Philos. Mag. 89 (2009) 907-923.

[86] D. Sweeney, C. Vest, Measurement of three-dimensional temperature fields above heated surfaces by holographic interferometry, Int. J. Heat Mass Transf. (1974).

[87] T.D. P., J.T. R., B.-C.P. J., Analysis of holographic fringe data using the dual reference approach, 30 (1991).

[88] D.-Y. Zhang, H.-C. Zhou, Temperature measurement by holographic interferometry for non-premixed ethylene-air flame with a series of state relationships, Fuel. 86 (2007) $1552-1559$.

[89] Convective stability of multicomponent fluids in the thermogravitational column, Phys. Rev. E. 79 (2009) 26308.

[90] P. Naumann, A. Martin, H. Kriegs, M. Larrañaga, M.M. Bou-Ali, S. Wiegand, Development of a thermogravitational microcolumn with an interferometric contactless detection system., J. Phys. Chem. B. 116 (2012) 13889-97. 
[91] J.K. Platten, The Soret Effect: A Review of Recent Experimental Results, J. Appl. Mech. 73 (2006) 5.

[92] T. Pollak, W. Koehler, Critical assessment of diffusion coefficients in semidilute to concentrated solutions of polystyrene in toluene, J. Chem. Phys. 130 (2009) 124905.

[93] S.A. Putnam, D.G. Cahill, Micron-scale apparatus for measurements of thermodiffusion in liquids, Rev. Sci. Instrum. 75 (2004) 2368-2372.

[94] A. Khoshnevis, A. Ahadi, M.Z. Saghir, Influence of static and oscillatory gravity fields on thermodiffusion of a binary mixture, Int. J. Therm. Sci. 75 (2014) 221-232.

[95] Q. Galand, Experimental investigation of the diffusive properties of ternary liquid systems, PhD Thesis, Univ. Bruxelles, Ec. Polytech. Bruxelles. 1 (2012).

[96] C. Pike, Two-Dimensional Fourier Processing of Rasterised Audio, (2008) Abstract A comprehensive software tool has been de.

[97] B. Heise, Image Processing for Phase-Sensitive Optical Coherence Tomography, 3838120582nd ed., Paperback, 2010.

[98] R. Vander, S.G. Lipson, I. Leizerson, Fourier fringe analysis with improved spatial resolution, Appl.Opt. 42 (2003) 6830-6837.

[99] M. Bahri, E.S.M. Hitzer, R. Ashino, R. Vaillancourt, Windowed Fourier transform of twodimensional quaternionic signals, Appl. Math. Comput. 216 (2010) 2366.

[100] T.J. Chung, Computational Fluid Dynamics, Cambridge University Press, 2010.

[101] T.R. Judge, Automatic Analysis of Interferometric Data - FRAN (Description and User Guide), 1996.

[102] L. Lepetit, G. Chériaux, M. Joffre, Linear techniques of phase measurement by femtosecond spectral interferometry for applications in spectroscopy, J.Opt.Soc.Am.B. 12 (1995) 2467-2474.

[103] W.W.M. Jr., Two-dimensional fringe-pattern analysis, Appl.Opt. 22 (1983) 3898-3901.

[104] E.A. Robinson, A historical perspective of spectrum estimation, Proc. IEEE. 70 (1982) 885-907.

[105] T.R. Judge, P.J. Bryanston-Cross, A review of phase unwrapping techniques in fringe analysis, Opt. Lasers Eng. 21 (1994) 199-239. 
[106] J.W. Cooley, J.W. Tukey, An algorithm for the machine calculation of complex Fourier series, Math. Comput. 19 (1965) 297-301.

[107] Liquid entrainment by gas flow along the interface of a liquid bridge, Eur. Phys. J. Spec. Top. 192 (2011) 63.

[108] D. Garcia, Robust smoothing of gridded data in one and higher dimensions with missing values, Comput. Stat. Data Anal. 54 (2010) 1167-1178.

[109] V. Shevtsova, A. Mialdun, D. Melnikov, I. Ryzhkov, Y. Gaponenko, Z. Saghir, et al., The IVIDIL experiment onboard the ISS: Thermodiffusion in the presence of controlled vibrations, Comptes Rendus Mec. 339 (2011) 310.

[110] A. Kianian, M.Z. Saghir, A. Ahadi, Experimental evidence of low Rayleigh vibration on mixture during thermodiffusion experiment, in: CASI ASTRO Quebec City April 23rd 26th Quebec City, 2012: p. 33.

[111] S. Mazzoni, DSC as part of the DCMIX project Experiment Scientific Requirements, 2011.

[112] A. Ahadi, A. Kianian, M.Z. Saghir, Heat and mass transport phenomena under influence of vibration using a new aided image processing approach, Int. J. Therm. Sci. 75 (2014) 233-248.

[113] H. Abdul-Rahman, Three dimensional Fourier fringe analysis and phase unwrapping, Liverpool John Moores University for the degree of Doctor of Philosophy, 2007.

[114] A. Mialdun, V.M. Shevtsova, Development of optical digital interferometry technique for measurement of thermodiffusion coefficients, Int. J. Heat Mass Transf. 51 (2008) 3164.

[115] H.W. Pritchard, P.B. Tompsett, K.R. Manger, Development of a thermal time model for the quantification of dormancy loss in Aesculus hippocastanum seeds, Seed Sci. Res. 6 (1996) 127-135.

[116] A.E. Kennelly, The Thermal Time Constants of Dynamo-Electric Machines, Trans. Am. Inst. Electr. Eng. XLIV (1925) 137-154.

[117] P.K.L. Chan, K.P. Pipe, Z. Mi, J. Yang, P. Bhattacharya, D. Lüerßen, Thermal relaxation time and heat distribution in pulsed InGaAs quantum dot lasers, Appl. Phys. Lett. 89 (2006) 011110.

[118] J. Ordóñez-Miranda, J.J. Alvarado-Gil, Thermal wave oscillations and thermal relaxation time determination in a hyperbolic heat transport model, Int. J. Therm. Sci. 48 (2009) 2053-2062. 
[119] A. Ahadi, A. Khoshnevis, M.Z. Saghir, Windowed Fourier Transform as an Essential Digital Interferometry Tool to Study Coupled Heat and Mass Transfer, Opt. Laser Technol. (2013).

[120] A. Mialdun, V. Shevtsova, Open questions on reliable measurements of Soret coefficients, Microgravity Sci. Technol. 21 (2008) 31-36.

[121] T.V. Vorburger, E.C. Teague, Optical techniques for on-line measurement of surface topography, Precis. Eng. 3 (1981) 61-83.

[122] J. Wolkoff, D.A. Woodward, A.J. Strecok, A thermocouple for the measurement of the surface temperature of a liquid metal, Chem. Eng. Sci. 21 (1966) 895-903.

[123] P.R.N. Childs, Advances in temperature measurement, Adv. Heat Transf. 36 (2003) 111181.

[124] G.P. Dubey, N. Tripathi, S.C. Bhatia, Refractive index of ternary liquid systems of squalane ( + hexane + benzene+ cyclohexane + benzene and + hexane + cyclohexane ), 43 (2005) 175-179.

[125] V. Alonso, J.A. González, I.G. de la Fuente, J.C. Cobos, Dielectric and refractive index measurements for the systems 1-pentanol+2,5,8,11,14-pentaoxapentadecane, or for 2,5,8,11,14-pentaoxapentadecane+octane at $(293.15-303.15) \mathrm{K}$, Thermochim. Acta. 551 (2013) 70-77.

[126] Q. Wen, J. Shen, Z. Shi, E. Dy, K.H. Michaelian, C. Fairbridge, et al., Temperature coefficients of the refractive index for hydrocarbons and binary mixtures, Chem. Phys. Lett. 539-540 (2012) 54-57.

[127] G.Z. Gershuni, D. Lyubimov, Thermal vibrational convection, John Wiley Sons. (1998) 358.

[128] G.Z. Gershuni, A.K. Kolesnikov, J.-C. Legros, B.I. Myznikova, On the vibrational convective instability of a horizontal, binary-mixture layer with Soret effect, J. Fluid Mech. 330 (n.d.) 251-269.

[129] G.Z. Gershuni, A.K. Kolesnikov, J.C. Legros, B.L. Myznikova, On the Vibrational Convective Instability of a Horizontal Binary-Mixture Layer with Soret Effect Under Transversal High Frequency Vibration., Int. J. Heat Mass. 42 (1999) 547.

[130] R. Savino, M. Lappa, Assessment of thermovibrational theory: application to g-jitter on the Space Station, J. Spacecr. Rockets. 40 (2003) 201-210.

[131] V. Shevtsova, Z. Saghir, T. Lyubimova, D. Melnikov, A. Mialdun, R. Ilya, 1-Year PostFlight Science ReportInfluence of vibrations on diffusion in liquids (IVIDIL), (2011). 
[132] M. Lappa, SODI-IVIDIL Experiment, 2010.

[133] S. Mazzoni, V. Shevtsova, A. Mialdun, D. Melnikov, Y. Gaponenko, T. Lyubimova, et al., Vibrating liquids in space, Europhys. News. 41 (2010) 14-16.

[134] Y. Yan, A. Viviani, M.Z. Saghir, Double diffusion convection under sinusoidal modulations of low-frequency vibrations, Acta Astronaut. 63 (2008) 665-672.

[135] V. Shevtsova, D. Melnikov, J.C. Legros, Y. Yan, Z. Saghir, T. Lyubimova, et al., Influence of vibrations on thermodiffusion in binary mixture: A benchmark of numerical solutions, Phys. Fluids. 19 (2007) 17111.

[136] T. Lyubimova, E. Shklyaeva, J.-C. Legros, V. Shevtsova, B. Roux, Numerical study of high frequency vibration influence on measurement of Soret and diffusion coefficients in low gravity conditions, Adv. Sp. Res. 36 (2005) 70-74.

[137] M. Takeda, H. Ina, S. Kobayashi, Fourier transform methods of fringe-pattern analysis for computer-based topography and interferometry, J.Opt.Soc.Am. 72 (1982) 156-160.

[138] C. Quan, H. Niu, C.J. Tay, An improved windowed Fourier transform for fringe demodulation, Opt. Laser Technol. 42 (2010) 126-131.

[139] E.L. Cussler, Diffusion: Mass Transfer in Fluid Systems, Cambridge University Press, 2009.

[140] A. Leahy-Dios, M.M. Bou-Ali, J.K. Platten, A. Firoozabadi, Measurements of molecular and thermal diffusion coefficients in ternary mixtures, J. Chem. Phys. 122 (2005) 234502.

[141] R.J. Riobóo, M. Philipp, M. a Ramos, J.K. Krüger, Concentration and temperature dependence of the refractive index of ethanol-water mixtures: influence of intermolecular interactions., Eur. Phys. J. E. Soft Matter. 30 (2009) 19-26.

[142] F. Croccolo, F. Plantier, G. Galliero, G. Pijaudier-Cabot, M.Z. Saghir, F. Dubois, et al., Note: Temperature derivative of the refractive index of binary mixtures measured by using a new thermodiffusion cell., Rev. Sci. Instrum. 82 (2011) 126105.

[143] F. Croccolo, M.-A. Arnaud, D. Bégué, H. Bataller, Concentration dependent refractive index of a binary mixture at high pressure., J. Chem. Phys. 135 (2011) 034901.

[144] A. Ahadi, M.Z. Saghir, An extensive heat transfer analysis using Mach Zehnder Interferometry during thermodiffusion experiment on board the International Space Station, Appl. Therm. Eng. (2013). 
[145] A. Ahadi, M.Z. Saghir, Experimental study of the impacts of forced vibration on thermodiffusion phenomenon in microgravity environment, Appl. Therm. Eng. 60 (2013) 348-358.

[146] Q. Kemao, Windowed Fourier transform for fringe pattern analysis, Appl.Opt. 43 (2004) 2695.

[147] R. Riquelme, I. Lira, C. Pérez-López, J.A. Rayas, Rodríguez-VerRamón, Interferometric measurement of a diffusion coefficient: comparison of two methods and uncertainty analysis, J. Phys. D. Appl. Phys. 40 (2007) 2769.

[148] V. Alvarez-Valado, H. Gonzalez-Jorge, B. V Dorrao, M. Miranda, F. Rodraguez, J.L. Valencia, et al., Testing phase-shifting algorithms for uncertainty evaluation in interferometric gauge block calibration, Metrologia. 46 (2009) 637.

[149] A. Federico, G.H. Kaufmann, Phase Retrieval in Digital Speckle Pattern Interferometry by Use of a Smoothed Space-Frequency Distribution, Appl.Opt. 42 (2003) 7066-7071.

[150] Designing Robust Preparative Purification Processes with High Performance, Chem. Eng. Technol. 31 (2008) 875.

[151] R. Khoshrooz, A.M. Vahid, M. Mirshams, M.R. Homaeinezhad, A. Ahadi, Novel method on using evolutionary algorithms for PD optimal tuning, Appl. Mech. Mater. 110-116 (2012) 4977-4984.

[152] A. Mialdun, V. Shevtsova, Digital interferometry as a powerful tool to study the thermodiffusion effect, Comptes Rendus Mec. 339 (2011) 362-368.

[153] V. Shevtsova, G. Galliero, A. Mialdun, V. Yasnou, Measurement of isothermal diffusion coefficients in ternary mixtures using counter flow diffusion cell, Comptes Rendus Mécanique. 341 (2013) 462-468.

[154] A. Mialdun, V. Shevtsova, Measurement of the Soret and diffusion coefficients for benchmark binary mixtures by means of digital interferometry, J. Chem. Phys. 134 (2011) 44524 .

[155] A. Mialdun, C. Minetti, Y. Gaponenko, V. Shevtsova, F. Dubois, Analysis of the Thermal Performance of SODI Instrument for DCMIX Configuration, Microgravity Sci. Technol. 25 (2013) 83-94.

[156] T.J. Jaber, H. Bataller, M.Z. Saghir, Measurement of Thermodiffusion Coefficient for Binary Hydrocarbon Fluid Mixtures in Porous Medium: Experimental and Numerical Results, ASME Conf. Proc. 2009 (2009) 461-469. 